\title{
Ultrafast Infrared Studies of Chemical Reaction Dynamics in Room-Temperature Liquids
}

\author{
Haw Yang \\ Ph.D. Thesis \\ Department of Chemistry \\ University of California, Berkeley \\ and \\ Chemical Sciences Division \\ Ernest Orlando Lawrence Berkeley National Laboratory \\ University of California \\ Berkeley, CA 94720
}

November 1999

This work was supported by the Director, Office of Science, Office of Basic Energy Sciences, Chemical Sciences Division, of the U.S. Department of Energy under Contract No. DE-AC03-76SF00098. 


\section{DISCLAIMER}

This report was prepared as an account of work sponsored by an agency of the United States Government. Neither the United States Government nor any agency thereof, nor any of their employees, make any warranty, express or implied, or assumes any legal liability or responsibility for the accuracy, completeness, or usefulness of any information, apparatus, product, or process disclosed, or represents that its use would not infringe privately owned rights. Reference herein to any specific commercial product, process, or service by trade name, trademark, manufacturer, or otherwise does not necessarily constitute or imply its endorsement, recommendation, or favoring by the United States Government or any agency thereof. The views and opinions of authors expressed herein do not necessarily state or reflect those of the United States Government or any agency thereof. 


\section{DISCLAIMER}

Portions of this document may be illegible in electronic image products. Images are produced from the best available original document. 


\title{
Ultrafast Infrared Studies of Chemical Reaction Dynamics in Room- Temperature Liquids
}

\author{
Copyright $\odot 1999$
}

by

Haw Yang

The U.S. Department of Energy has the right to use this document for any purpose whatsoever including the right to reproduce all or any part thereof. 


\title{
ULTRAFAST INFRARED STUDIES OF CHEMICAL REACTION DYNAMICS IN ROOM-TEMPERATURE LIQUIDS
}

\author{
by \\ Haw Yang
}

B. S. (National Taiwan University) 1991

A dissertation submitted in partial satisfaction of the

requirements for the degree of

Doctor of Philosophy

in

Chemistry

in the

GRADUATE DIVISION

of the

UNIBSERSITY OF CALIFORNIA, BERKELEY

Committee in charge:

Professor Charles B. Harris, Chair

Professor Robert G. Bergman

Professor Roger Falcone

Fall 1999 


\begin{abstract}
Ultrafast Infrared Studies of Chemical Reaction Dynamics in Room-Temperature Liquids

by
\end{abstract}

Haw Yang

Doctor of Philosophy in Chemistry

University of California at Berkeley

Professor Charles B. Harris, Chair

Femtosecond infrared spectroscopy provides sufficient spectral and temporal resolution to support a detailed investigation of the early events of a photochemical reaction. Previously unreported transient species that arise as intermediates during the course of a reaction may have lifetimes that are too short for conventional characterization. For these species, quantum-mechanical (density functional theoretical and $a b$ initio) electronic structure calculations provide invaluable insight into chemical properties including molecular structure and energetics. With the combination of experimental and theoretical results, it is possible to assemble a comprehensive picture of the reaction dynamics of a system that is intricately influenced by the surrounding solvent molecules.

The mechanisms of several important organometallic reactions, such as alkane C$\mathrm{H}$ bond activation by $\eta^{3}-\mathrm{Tp}{ }^{*} \mathrm{Rh}(\mathrm{CO})$, silane $\mathrm{Si}-\mathrm{H}$ bond activation by $\eta^{5}-\mathrm{CpMn}(\mathrm{CO})_{2}$ and $\eta^{5}-\mathrm{CpRe}(\mathrm{CO})_{2}$, as well as chlorinated methane $\mathrm{C}-\mathrm{Cl}$ bond cleavage by the $\operatorname{Re}(\mathrm{CO})_{5}$ radical are elucidated. The results demonstrate the importance of molecular morphology change ( $\mathrm{C}-\mathrm{H}$ and $\mathrm{Si}-\mathrm{H}$ activation), solvent rearrangement ( $\mathrm{Si}-\mathrm{H}$ activation), intersystem crossing ( $\mathrm{Si}-\mathrm{H}$ activation), and solvent caging $(\mathrm{C}-\mathrm{Cl}$ cleavage) in understanding the 
reactivity of the organometallic species. The nature of the apparent free-energy barrier for $\mathrm{C}-\mathrm{H}, \mathrm{Si}-\mathrm{H}$, and $\mathrm{C}-\mathrm{Cl}$ bond activation reaction is found to be cleavage of an alkane $\mathrm{C}-\mathrm{H}$ bond, rearrangement of a silane molecule $\mathrm{HSiR}_{3}(\mathrm{R}=$ alkyl group) from a nonreactive alkyl site to the reactive $\mathrm{Si}-\mathrm{H}$ bond, and $\mathrm{Cl}$ atom transfer from a chlorinated methane molecule to $\operatorname{Re}(\mathrm{CO})_{5}$, respectively. These results support previous $a b$ initio calculations for $\mathrm{C}-\mathrm{H}$ and $\mathrm{Si}-\mathrm{H}$ bond activation reaction profiles which suggest that cleavage of an alkane $\mathrm{C}-\mathrm{H}$ bond by a transition metal center, unlike that of a silane $\mathrm{Si}-\mathrm{H}$ bond, involves a precursor complex. The results of $\mathrm{C}-\mathrm{Cl}$ bond activation by a transitionmetal radical call for a re-examination of existing models that invoke an intermediate with either nineteen valence electrons at the metal center or charge-transfer character. 
To my family:

Chih-Mo Yang, Hsiu-Ching Tseng, and Chia-Yi Yang 


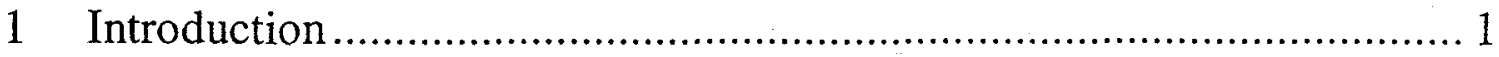

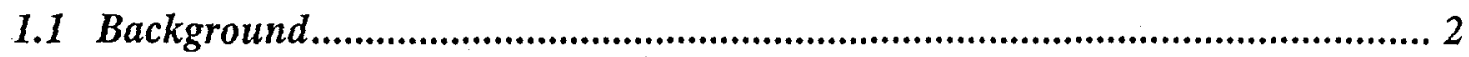

1.2 Dissertation Overview.................................................................................... 5

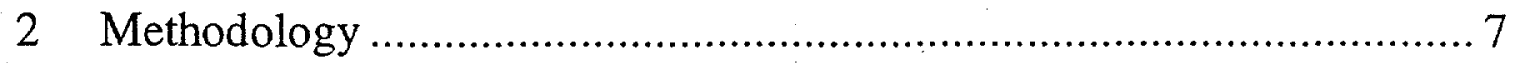

2.1 Instrumentation of Femtosecond Infrared Spectroscopy ..................................... 8

2.2 IR Focal Plane Array (IR-FPA) Detector ............................................................15

2.3 Frequency Chirping at the Vicinity of a Strongly-Absorbing IR Band .............17

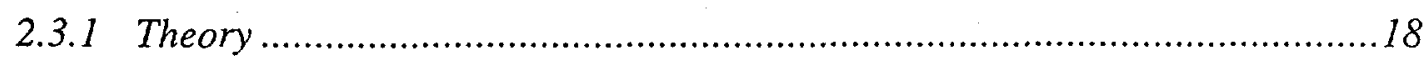

2.3.2 Comparison with Experiments............................................................22

2.4 The Vibrational Stark Effect ........................................................................23

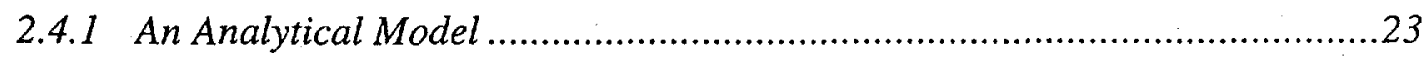

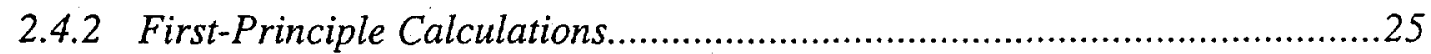

2.5 Classical Molecular Dynamics Simulation ........................................................30

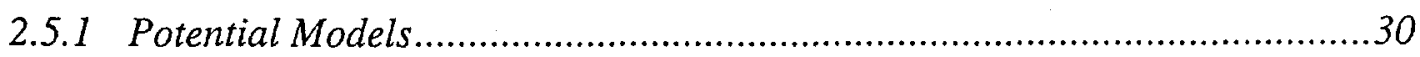

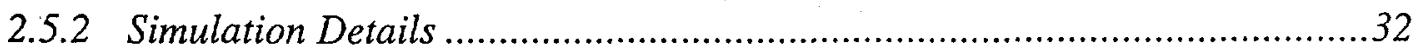

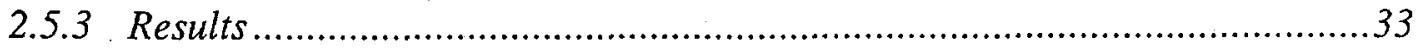

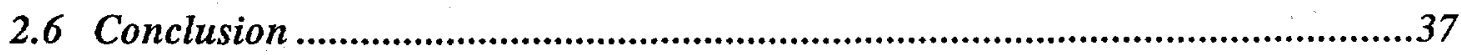

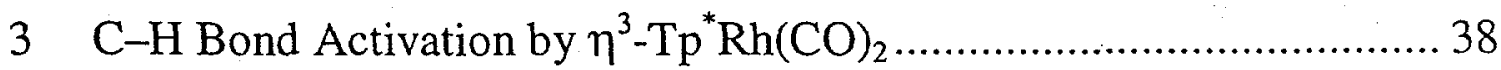

3.I The Dynamics of Reaction Intermediates-Vibrational Relaxation and Molecular Morphology Change .............................................................................39

3.2 The Activation Barrier-the Bond-Breaking Step …...........................................42

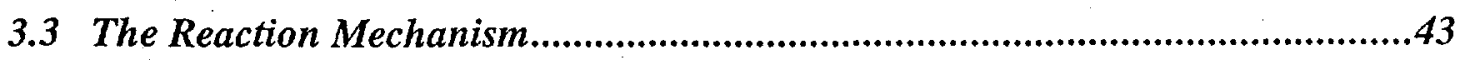

4 Femtosecond Infrared Studies of Si-H Bond Activation by $\eta^{5}$ -

$\mathrm{CpMn}(\mathrm{CO})_{3}$ 46 


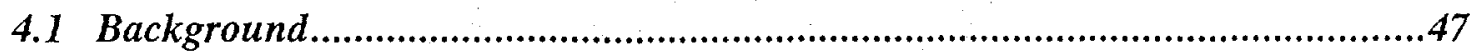

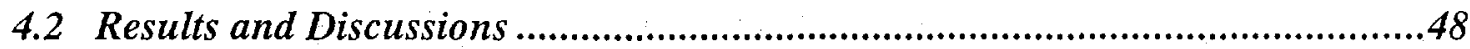

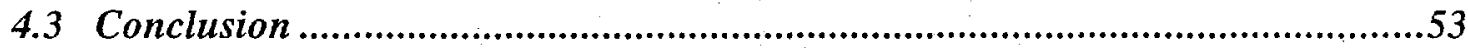

5 The reaction mechanism of $\mathrm{Si}-\mathrm{H}$ bond activation studied using femtosecond to nanosecond IR spectroscopy and ab initio methods ... 55

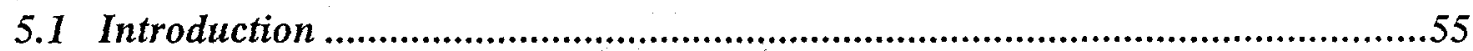

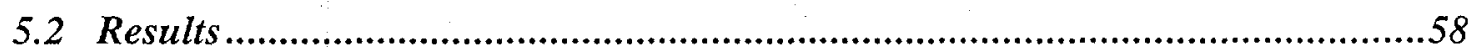

5.2.1 Activation of the Silicon-Hydrogen Bond of Et $t_{3} \mathrm{SiH}$ by $\mathrm{CpMn}(\mathrm{CO})_{3} \ldots \ldots \ldots . . . .58$

5.2.2 Activation of the Silicon-Hydrogen Bond of Et $t_{3} \mathrm{SiH}$ by $\mathrm{CpRe}(\mathrm{CO})_{3} \ldots \ldots \ldots \ldots . . . .63$

5.2 .3 ab initio Calculation Results ...........................................................70

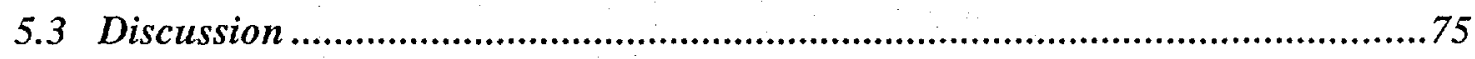

5.3.1 Activation of the Silicon-Hydrogen Bond of $\mathrm{Et}_{3} \mathrm{SiH}$ by $\mathrm{CpMn}(\mathrm{CO})_{3} \ldots \ldots \ldots . . . .76$

5.3.2 Activation of the Silicon-Hydrogen Bond of $\mathrm{Et}_{3} \mathrm{SiH}$ by $\mathrm{CpRe}(\mathrm{CO})_{3} \ldots \ldots \ldots . . . . .82$

5.4 Closing Remarks ................................................................................ 84

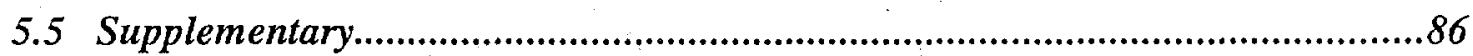

6 Femtosecond Infrared Studies of a Prototypical One-Electron OxidativeAddition Reaction: Chlorine Atom Abstraction by the $\operatorname{Re}(\mathrm{CO})_{5}$ Radical.

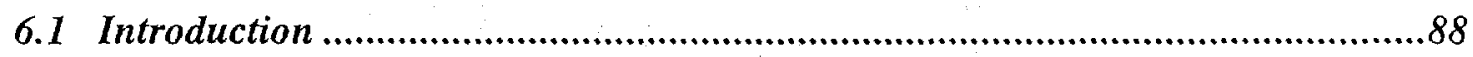

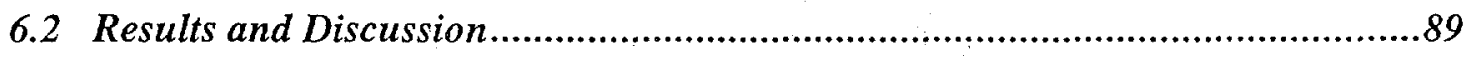

6.3 Conclusion ......................................................................................93

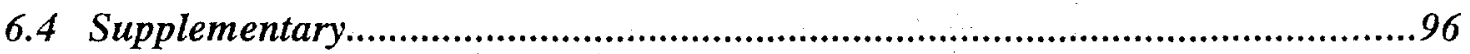

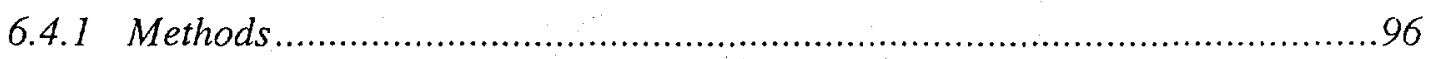

6.4.2 Diffusion Model for Geminate Recombination of $\operatorname{Re}(\mathrm{CO})_{5}$ Radicals............98

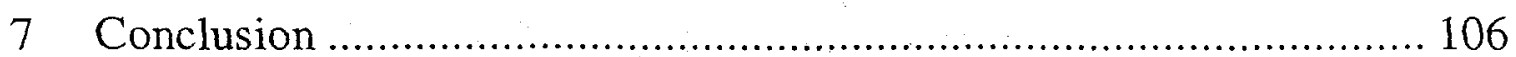

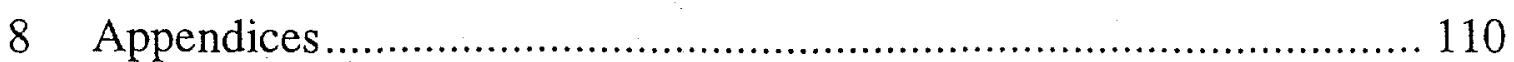


8.1 Example Input Files for the Quantum Chemical Calculation Package GAMESS

8.1.1 The Input File for Calculating the CASSCF-PT2 Energy of the Singlet $\mathrm{CpMn}(\mathrm{CO})_{2}$.

8.1.2 The Input File for Calculating the CASSCF-PT2 Energy of the Triplet $\operatorname{CpMn}(\mathrm{CO})_{2}$.

8.2 Quantitative Considerations of the Triplet-Singlet Intersystem Crossing in Coordinatively Unsaturated Organometallic Compounds. 114

8.2.1 A Summary of Theoretical Models 115

8.2.2 The Input File for Calculating the Spin-Orbital Coupling Constant of $\mathrm{CpMn}(\mathrm{CO})_{2}$ Using MCSCF Wavefunctions

8.2.3 A MATLAB Script for Calculating the Overlap of Vibrational Wavefunctions between the Singlet and Triplet States.

9 Bibliography 125 


\section{List of Figures}

Figure 1.1 An illustration of one- and two-electron oxidative addition. ....................... 2

Figure 1.2 A bonding model for a dihydrogen metal complex................................ 3

Figure 1.3 A schematic illustration of nineteen-electron intermediate in one-electron

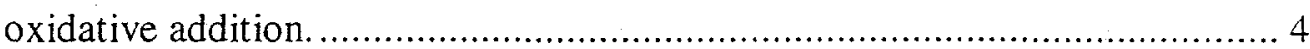

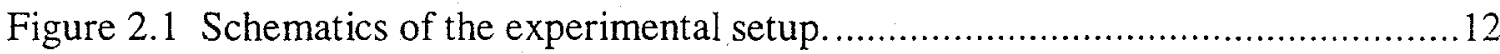

Figure 2.2 Auto-correlation plots of the three laser pulses. ...................................13

Figure 2.3 Spectral analyses of the ultrafast pulses...........................................14

Figure 2.4 Schematics of the IR-array setup..................................................... 16

Figure 2.5 Comparison of experiments with theory for pump induced IR chirping. ......22

Figure 2.6 Evaluation of $a b$ initio vibrational Stark effect for CO. ............................27

Figure 2.7 Evaluation of ab initio vibrational Stark effect for $\mathrm{CH}_{3} \mathrm{CN}$.....................28

Figure 2.8 Evaluation of $a b$ initio vibrational Stark effect for $\mathrm{C}_{6} \mathrm{H}_{5} \mathrm{CN}$. ..................29

Figure 2.9 Characterization of a MD simulation engine for $\mathrm{CH}_{3} \mathrm{CN}$........................34

Figure 2.10 Non-equilibrium MD simulation of dipole solvation for a sudden dipole

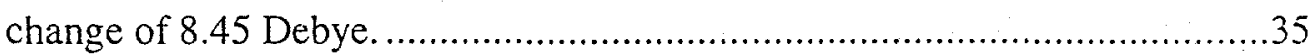

Figure 2.11 Non-equilibrium MD simulation of dipole solvation for a sudden dipole

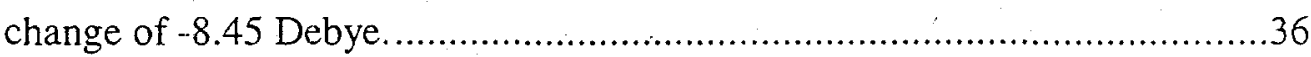

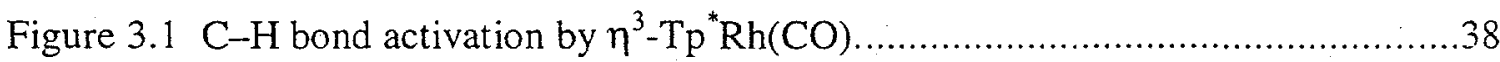

Figure 3.2 Ultrafast IR spectra of $\eta^{3}-\mathrm{Tp} * \mathrm{Rh}(\mathrm{CO})_{2}$ in alkane solution following $295-\mathrm{nm}$ excitation.

Figure 3.3 Ultrafast kinetics of $\eta^{3}-\mathrm{Tp} * \mathrm{Rh}(\mathrm{CO})_{2}$ in alkane solution..........................41

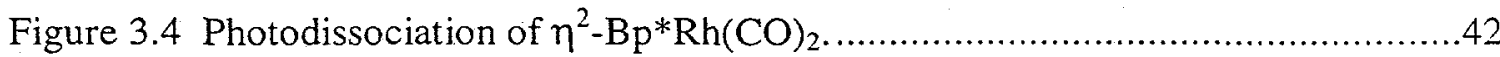

Figure 3.5 Nanosecond kinetics of $\eta^{3}-\mathrm{Tp} * \mathrm{Rh}(\mathrm{CO})_{2}$ in alkane solution. ......................43

Figure 3.6 A proposed reaction mechanism for the alkane $\mathrm{C}-\mathrm{H}$ bond activation by $\eta^{3}-\mathrm{Tp} * \mathrm{Rh}(\mathrm{CO})_{2}$ covering the ultrafast dynamics to nanosecond kinetics......44

Figure 4.1 A bonding model for $\mathrm{C}-\mathrm{H}$ bond $\sigma$-comple. .......................................46 
Figure 4.2 Ultrafast IR spectra of CpMn(CO) ${ }_{3}$ in triethylsilane following 295-nm photolysis.

Figure 4.3 Ultrafast IR kinetics of $\mathrm{CpMn}(\mathrm{CO})_{3}$ in triethylsilane following $295-\mathrm{nm}$ photolysis.

Figure 4.4 A proposed reaction mechanism for the photochemical $\mathrm{Si}-\mathrm{H}$ bond activation of $\mathrm{Et}_{3} \mathrm{SiH}$ by $\mathrm{CpMn}(\mathrm{CO})_{3}$. Suggestive chemical structures are in brackets. ..53

Figure 5.1 IR band assignments of chemical species involved in $\mathrm{S}-\mathrm{H}$ bond activation by $\mathrm{CpM}(\mathrm{CO})_{2}, \mathrm{M}=\mathrm{Mn}, \mathrm{Re}$

Figure 5.2 Ultrafast IR spectra of $\mathrm{CpMn}(\mathrm{CO})_{3}$ in triethylsilane following $325-\mathrm{nm}$ excitation.

Figure 5.3 Ultrafast IR kinetics of $\mathrm{CpMn}(\mathrm{CO})_{3}$ in triethylsilane following $325-\mathrm{nm}$ excitation.

Figure 5.4 Nanosecond kinetics of $\mathrm{CpMn}(\mathrm{CO})_{3}$ in neat triethylsilane following $295-\mathrm{nm}$ excitation.

Figure 5.5 Ultrafast IR spectra of $\mathrm{CpRe}(\mathrm{CO})_{3}$ in triethylsilane following 295-nm excitation.

Figure 5.6 Ultrafast $\cdot \mathrm{IR}$ kinetics of $\mathrm{CpRe}(\mathrm{CO})_{3}$ in triethylsilane following $295-\mathrm{nm}$ excitation .66

Figure 5.7 Modeling of the observed transient traces of $\mathrm{CpRe}(\mathrm{CO})_{3}$ 68

Figure 5.8 Nanosecond kinetics of $\mathrm{CpRe}(\mathrm{CO})_{3}$ following 295 -nm excitation.

Figure 5.9 Geometries of the parent molecules, the ethane-solvate, and the final product optimized at the MP2 / lanl2dz level of theory.

Figure 5.10 Side view and top view for the MP2 / lan12dz optimized geometries of the 16-e- species. .72

Figure 5.11 UV/ Vis spectra of $\mathrm{CpMn}(\mathrm{CO})_{3}$ and $\mathrm{CpRe}(\mathrm{CO})_{3}$. .76

Figure 5.12 Comparison of ultrafast kinetics of $\mathrm{CpMn}(\mathrm{CO})_{3}$ at different pump wavelengths. .78

Figure 5.13 Proposed reaction mechanism for the silicon-hydrogen bond activation by $\mathrm{CpMn}(\mathrm{CO})_{3}$ covering the ultrafast dynamics to nanosecond kinetics. 
Figure 5.14 Proposed reaction mechanism for the silicon-hydrogen bond activation by $\mathrm{CpRe}(\mathrm{CO})_{3}$ covering the ultrafast dynamics to microsecond kinetics.

Figure 6.1 Previously proposed reaction schemes for $\mathrm{Cl}$ atom abstraction by $\operatorname{Re}(\mathrm{CO})_{5} . .89$

Figure 6.2 Transient difference spectra in the $\mathrm{CO}$ stretching region for $\mathrm{Re}_{2}(\mathrm{CO})_{10}$ in neat

$\mathrm{CCl}_{4}$ and hexanes. .90

Figure 6.3 Fits of ultrafast kinetics to a diffusion model. .91

Figure 6.4 DFT structures for the final product and transition-state structures. .94

Figure 6.5 Detailed fitting of ultrafast kinetics to a diffusion model. 104

Figure 6.6 Nanosecond kinetics of $\mathrm{Cl}$-atom abstraction reaction. .105

Figure 7.1 An illustration for solvation / spin crossover process. .107

Figure 8.1 Illustraion of Landau-Zener spin crossover. .116

Figure 8.2 Graphical representation of the vibrational quantum numbers that contribute to the spin crossover rate. 


\section{List of Tables}

Table 2.1 Selected results for characterization of different ab initio approaches to calculating the vibrational tuning rate.

Table 2.2 Intermolecular parameters for acetonitrile MD simulation.

Table 5.1. Critical bond distances (in $\AA$ ) of the important structures for the Si-H bond activation reactions optimized at the MP2 / lanl2dz level of theory. ${ }^{a}$

Table 5.2. Calculated MP2 / lanl2dz binding energies (in $\mathrm{kcal} / \mathrm{mol}$ ) for singlet $\eta^{5}-\mathrm{CpM}(\mathrm{CO})_{2} \mathrm{~L}, \mathrm{M}=\mathrm{Mn}, \mathrm{Re}^{\mathrm{a}}$ .74

Table 5.3. Comparison of the energy difference, $\Delta \mathrm{E}=\mathrm{E}$ (singlet)- $\mathrm{E}$ (triplet), between the singlet and triplet $\eta^{5}-\mathrm{CpM}(\mathrm{CO})_{2}$ calculated using different methods (in $\mathrm{kcal} / \mathrm{mol}$ )

Table 6.1. Summary of Electron-Population Analysis for the $\mathrm{Re}$ and $\mathrm{Cl}$ Centers Involved in the Reaction of Cl-Atom Abstraction by the $\operatorname{Re}(\mathrm{CO})_{5}$ Radical. 103 


\section{Acknowledgment}

I am grateful for having had the privilege of working with excellent colleagues and for having made so many good friends during my years at Berkeley. I would like to thank Charles Harris for creating a stimulating research environment in which his group members enjoy great intellectual latitude. Charles showed me, through casual conversions and by his example, how to define and approach a problem and then to look at it from an entirely different perspective. Tim Lian taught me femtosecond infrared spectroscopy in his two-year stay at Berkeley, from construction of the laser system to carrying out experiments. Since then, he has continued to give me advises and sometimes kind reproof, both as a friend and as a mentor. In my early days as a graduate student studying $\mathrm{C}-\mathrm{H}$ bond activation, I had the good fortune of learning directly from one of the leading scientists in the field, Bob Bergman. I am especially grateful for Bob's enormous patience in teaching me how to express ideas rigorously. I am thankful to Heinz Frei for his guidance through the step-scan FTIR experiments and for his sharing the views about contemporary issues in science. I am also indebted to Roger Falcone, who was always ready to lend a helping hand at critical times, especially in the beginning of femtosecond IR projects. The outstanding technical and administrative personnel in the department, particularly Vijaya Narasimhan who was key in keeping the researches going in our group, were indispensable in the research. The work included in this dissertation could not have been possible without the financial support from the National Science Foundation and specialized equipment supported by U.S. Department of Energy.

The senior members, Steve Bromberg, Matt Asplund, Jason King, Robert Lingle, Robert Jordan, Nein-Hui Ge, Jason McNeill, and Steve Gadd provided the much needed help and guidance during my trying first year in graduate school. With their help, much of my scattered technical perception was consolidated to scientific understanding. Robert Lingle and Tim further sharpened my logical acuity through frequent spontaneous debates over various issues. Steve Bromberg and Robert Jordan was always willing to 
explain to me, with great patience, matters ranging from scientific concepts to hard-rock concerts. From Jason McNeill and Matt Asplund, I learned how to administrate a cluster of UNIX workstations. Ken Kotz and I collaborated fruitfully on various projects; jointly we strode past obstacles that had lied in the path of a project. Through him, I had the pleasure to befriend with P.J. Alaimo who, together with Bruce McNamara and Jake Yeston, showed me how to think from the viewpoint of an inorganic chemist. The research projects gained extra momentum from Preston Snee who seemed to take upon difficult tasks with smiles. Ken, Preston, Matt Wilkens, and later Christine Payne helped me better understand many issues that I once thought I understood. It was a great honor of mine to be able to work side by side with these talented scientists.

I would also like to thank Nien-Hui. Ge, Chung Wong, Kelly Gaffney, André Miller, and Simon Liu for spending time explaining to me their latest experimental results. Exchange of ideas with André, Simon, and later with Paul Szymanski continued either on a ski slope or around a campfire. Graduate school would have been less interesting were it not for the weekly shopping / luncheon / science forum with Chung. Like a spoiled child, I much enjoyed the generous friendship extended by Judy Kim, Mike Tauber, Travis Taylor, Melonie Hall and many other friends, who not only taught me science and beyond, but also infused my stint at Berkeley with warmth. Finally, I owe it to my Family who have fostered, and continued to support, my love for the Nature:

"Thanks to the human heart by which we live,

Thanks to its tenderness, its joys, and fears,

To me the meanest flower that blows can give

Thoughts that do often lie too deep for tears."

Stanza XI in Ode: Intimations of Immortality from Recollections of Early Childhood William Wordsworth, 1803-6. 


\section{INTRODUCTION}

When describing chemical dynamics, one usually refers to a simplified system consisting of only the reactive site and its immediate surroundings. The basis for conceptualizing chemical reactivity depends upon the knowledge of elementary steps that occur at the active site. The elementary steps include changes in electron distribution, molecular structure, translocation of chemical moieties, and solvent rearrangement to accommodate the new chemical environment. These processes are inevitably modulated by the surrounding media on time scales of picoseconds or less. Therefore, a critical test of our fundamental understanding of chemical reactivity requires experimental techniques that allow resolution of those ultrafast events. Femtosecond infrared (fs-IR) offers such an opportunity due to its ability to deliver chemically specific information.

The study of transition-metal mediated chemical reactions that are thought to take place at unsaturated metal sites is one problem that is amenable to the femtosecond infrared spectroscopic technique. Those reactions range from industrial process such as petroleum refining to biological cycles such as nitrogen fixation and photosynthesis. Ideally, one would like to observe the chemical events that evolve around such an active site as a reaction develops. This can be accomplished by using a $\mathrm{CO}$ ligand attached to

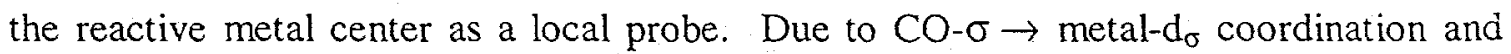
metal- $\mathrm{d}_{\pi} \rightarrow \mathrm{CO}-\pi^{*}$ back-donation, the infrared vibrational frequency of the CO ligand is a very sensitive measure of the chemical environment of the metal. Qualitatively, an increase (or decrease) of the metal electron density reduces (augments) the C-O bond order, resulting in a lower (higher) CO stretching frequency. Experimentally, an ultrafast UV pulse dissociates a photolabile ligand from an organometallic compound to result in a reactive intermediate with an unsaturated metal center. A cascade of chemical events, for example processes that ultimately lead to oxidative addition, then ensues. The thesis of 
this dissertation is the study of the important class of organometallic reactions-oxidative addition reactions-utilizing ferntosecond infrared spectroscopy. Since numerous excellent reviews have been given to this subject, only a brief account is presented below.

\subsection{Background}

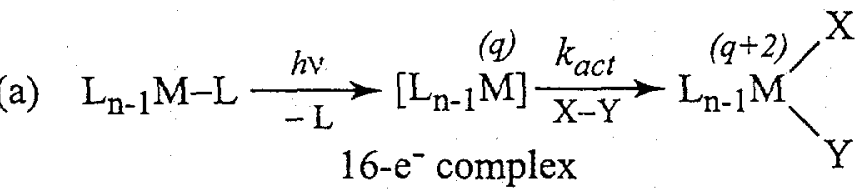

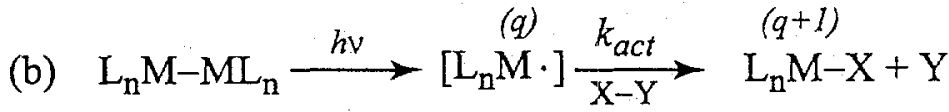

(1-electron) 17- $\mathrm{e}^{-}$complex

Figure 1.1 An illustration of one- and two-electron oxidative addition.

Transition metals capable of existing in multiple oxidation states may accept new ligands that increase the formal charge of the metal. Such translocations of atoms, molecules, or groups onto a metal center constitute a special class of reaction known as oxidative addition [1]. These reactions are of fundamental importance not only in inorganic chemistry, but also in organic synthesis, catalysis [2], and biological sciences [3]. The number of chemical moieties that is transferred to the metal categorizes this type of reaction into two-electron or one-electron oxidative addition, illustrated in Figure 1.1. One notable feature of the reaction patterns in Figure $\mathbf{1 . 1}$ is the weakening, or "activation," of an otherwise strong chemical bond (X-Y bond in the figure) to the extent that the reactions proceed under such mild conditions as biological environments. 


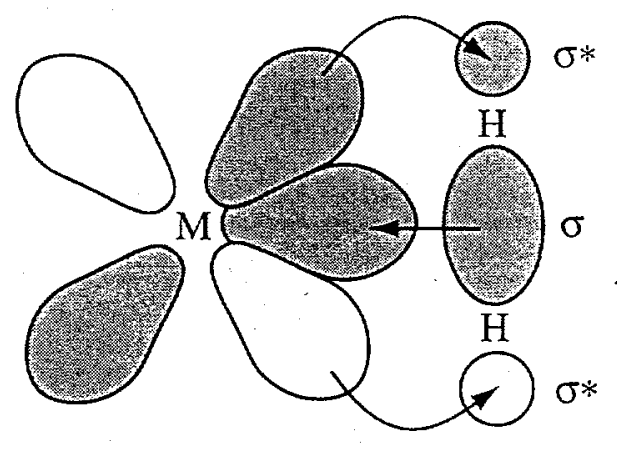

Figure 1.2 A bonding model for a dihydrogen metal complex.

For a two-electron oxidative-addition reaction, the weakening and eventual cleavage of a chemical bond can be understood from the bonding of dihydrogen transitional metal complexes such as $\mathrm{W}(\mathrm{CO})_{3}\left(\mathrm{PPri}_{3}\right)_{2}\left(\mathrm{H}_{2}\right)(\mathrm{Pri}=$ isopropyl) [4]. Figure 1.2 shows a qualitative molecular orbital (MO) picture for a dihydrogen complex $[5,6]$. Such a $\sigma$ complex is stabilized by two factors. On the one hand, the hydrogen molecule interacts with the metal center in a manner similar to a Lewis acid-base pair through electron donation from the filled $\mathrm{H}_{2} \sigma$-orbital to the empty metal- $\mathrm{d}_{\sigma}$ orbital. The same interaction also weakens, but does not break the $\mathrm{H}-\mathrm{H}$ bond. On the other hand, the metal-dihydrogen complexation is strengthened by electron "back-donation" from filled metal $d_{\pi}$ orbitals to the empty $\mathrm{H}_{2} \sigma^{*}$-orbital, which further weakens the $\mathrm{H}-\mathrm{H}$ bond. It follows that, from the MO viewpoint, the degree to which the $\mathrm{H}-\mathrm{H}$ bond is activated depends on the energetic and spatial overlaps between the metal and dihydrogen MOs. The concept of a precursor $\sigma$-complex has been extended to understanding the reactivity of other two-electron oxidative addition reactions, $\mathrm{C}-\mathrm{H}$ and $\mathrm{Si}-\mathrm{H}$ activation. In particular, the observed selectivity of primary $>$ secondary $>$ tertiary $\mathrm{C}-\mathrm{H}$ bonds in $\mathrm{C}-\mathrm{H}$ activation is attributed to a more stable (less sterically hindered) $\sigma$-complex intermediate for a primary $\mathrm{C}-\mathrm{H}$ bond in the reaction.

A substantial amount of indirect evidence in the literature has attested to the existence of a $\sigma$-complex intermediate in alkane $\mathrm{C}-\mathrm{H}$ bond activation [7]. Theoretical 
calculations also provide much insight into the possible involvement of a precursor intermediate in a two-electron oxidative-addition reaction. For example, Musaev and Morokuma have computed the reaction profiles for $\sigma$-bond activation of $\mathrm{H}_{2}, \mathrm{CH}_{4}, \mathrm{NH}_{3}$, $\mathrm{H}_{2} \mathrm{O}$, and $\mathrm{SiH}_{4}$ by $\eta^{5}-\mathrm{CpRh}(\mathrm{CO})\left(\mathrm{Cp}=\mathrm{C}_{5} \mathrm{H}_{5}\right)$. Their calculations predict a precursor complex in the activation of $\mathrm{C}-\mathrm{H}, \mathrm{N}-\mathrm{H}$, and $\mathrm{O}-\mathrm{H}$ bonds but not in the activation of $\mathrm{H}-\mathrm{H}$ or $\mathrm{Si}-\mathrm{H}$ bonds [8]. The marked difference along the calculated reaction coordinates for the isoelectronic $\mathrm{C}-\mathrm{H}$ and $\mathrm{Si}-\mathrm{H}$ bonds can be understood by the aforementioned $\mathrm{MO}$ picture. The Si d-orbitals allow a better interaction between the $\mathrm{Si}-\mathrm{H}$ bond and the metal d-orbitals such that the reaction proceeds to completion without passing through a precursor complex.

$$
\begin{aligned}
& \mathrm{L}_{\mathrm{n}} \mathrm{M}+: \ddot{\mathrm{X}}-\mathrm{CR}_{3} \longrightarrow \mathrm{L}_{\mathrm{n}} \mathrm{M} \odot \ddot{\mathrm{X}}-\mathrm{CR}_{3} \longrightarrow \mathrm{L}_{\mathrm{n}} \mathrm{M}-\mathrm{X}+\cdot \mathrm{CR}_{3} \\
& 17-\mathrm{e}^{-} \text {complex 19-e" complex }
\end{aligned}
$$

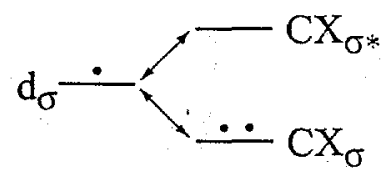

Figure 1.3 A schematic illustration of nineteen-electron intermediate in one-electron oxidative addition.

Along a parallel vein, it has also been proposed that a 19-electron intermediate may be involved in atom-abstraction reactions via one-electron oxidative addition to open-shell 17-electron organometallic compounds [9-12]. In a halogen-atom abstraction reaction, for example, the unpaired metal $d_{\sigma}$ orbital spatially overlaps with the halogen lone-pair orbital to form an intermediate that has 19 formal electrons in the coordination shell (Figure 1.3). Energetically, the metal $d_{\sigma}$ orbital interacts with the $\mathrm{C}-\mathrm{X} \sigma^{*}$ orbital, leading to reduction of the $\mathrm{C}-\mathrm{X}$ bond order and eventually resulting in cleavage of the $\mathrm{C}$ $\mathrm{X}$ bond [13]. 
It is evident in the light of the above discussion that intermediates play a critical role in oxidative-addition reactions. As such, a detailed knowledge of the dynamics of the intermediates is crucial for our fundamental understanding of oxidative addition reactions and of the nature of chemical bonds. Recently, development in this area has greatly accelerated owing to the increasingly sophisticated and efficient quantum chemical computational methods. Theoretical calculations provide valuable insight (e.g., the electronic structure and chemical bonding) for transient intermediates the lifetimes of which are too short for conventional characterization [14]. However, they also oftentimes rely on a priori knowledge of an approximate, sometimes idealized, reaction coordinate. Without experimental results, theoretical consideration may easily overlook such aspects as the dynamic partitioning of reaction pathways in the excited state or during solvation, such as those that occur in $\mathrm{Si}-\mathrm{H}$ bond activation which will be discussed later. Therefore, a close interplay between experiment and theory will be necessary in order to unveil the reaction dynamics underlying a complicated reaction.

\subsection{Dissertation Overview}

This dissertation includes the development, implementation, and application of techniques suitable for the study of liquid-phase reaction dynamics. Chapter 2 describes the instrumentation of the fs-IR spectrometer and aspects of signal analysis that have not been fully discussed in the literature. Also contained in Chapter 2 are the development and unpublished results of a classical molecular dynamics simulation engine that serves to statistically connect the molecular motions to the ensemble averaged experimental observables. Chapters 3 through 6 consist of published results utilizing these techniques to study a special class of reaction that is central to our understanding of inorganic chemistry and chemical bonds. They include the studies of the prototypical oxidativeaddition of the two-electron $\mathrm{C}-\mathrm{H}$ (Chapter 3) $[15,16]$ and $\mathrm{Si}-\mathrm{H}$ bonds (Chapters 4 and 5) $[17,18]$, and of the one-electron $\mathrm{Cl}$ atom (Chapter 6) [19] to organometallic compounds. Chapter 7 summarizes the main results that may be generalized to other classes of 
chemical reactions $[20,21]$. Finally, unpublished calculation details that may merit future studies are included in Chapter 8. 


\section{Methodology}

This chapter provides a general description of the techniques used for the study of liquid-phase reaction dynamics. Approaching chemical problems from a quantum mechanical perspective has now become a well-accepted tactic and has been routinely employed to augment experimental results in many laboratories. As such. densityfunctional theoretical and first-principle ( $a b$ initio) calculations play an integral part of the researches presented in this dissertation. Since all the calculation details will be explained in the individual chapters, they will not be repeated here. Nonetheless, certain types of computation, including the multi-configurational complete-active-space calculations, are more involved such that it is not obvious to compose proper input files. For those calculations, representative input scripts are included in the Appendices. During the course of the research, several computer programs have been developed. These include (1) a data-processing program written for collating spectral data taken with the single-element detectors, "spa" (1084 lines); ${ }^{1}$ (2) a non-linear data fitting program, "kinfit" (1722 lines); (3) a molecular simulation program that is capable of performing equilibrium or non-equilibrium simulations, "nemd" (6356 lines); and (4) a dataacquisition program, "spec" (5977 lines). ${ }^{1}$ Due to the length of these programs, they are not included in this chapter but printed out in a separate volume and archived in the laboratory.

The rest of the chapter is organized as follows. The configuration of the fs-IR setup is outlined in Section 2.1 to deliver a general idea of the experimental setup. Up-todate vendor information for system-specific components and maintenance notes are supplied at appropriate places. Section 2.2 discusses briefly the installation of the IR

\footnotetext{
${ }^{1}$ This program uses binary-tree data structure to process the data. Such a data structure frees the user from constraints of experimental setup including the number of scans and ordering of spectral (or temporal) scans.
} 
Focal Plane Array detector (IR-FPA), which allows simultaneous acquisition of an IR spectrum of $\sim 70 \mathrm{~cm}^{-1}$ on a shot-to-shot basis. Theoretical aspects in the femtosecond infrared spectroscopy that are not obvious in the relevant publications but may appear in the future research projects are discussed in Sections 2.3 and 2.4. Section 2.5 briefly introduces a molecular simulation engine. Some simulation results that may help to understand the solvent dynamics during the course of a chemical reaction are also presented and discussed in this section.

\subsection{Instrumentation of Femtosecond Infrared Spectroscopy}

The fs-IR spectrometer can be roughly divided into three parts: the oscillator, the dye-chain amplifier, and the detector. A schematic layout is displayed in Figure 2.1. Although the entire system spans three optical tables (the outlining boxes), one experiences very little alignment drift over the course of a one-week long experiment. As it will become clear in the next paragraph, the quality of the oscillator output dictates the performance of the entire system.

The solid-state Ti:sapphire oscillator (Murnane-Kapteyn design) is pumped by a commercial 5-W $\mathrm{Ar}^{+}$laser (Coherent Innova 310, all lines) to produce pulses of fullwidth-half-maximum (FWHM) $\sim 60 \mathrm{fs}$ at $\sim 800 \mathrm{~nm}$ with a repetition rate of $\sim 80 \mathrm{MHz}$ when it is mode locked. The $\sim 5-n J$ pulses ${ }^{2}$ are further amplified by a series of two prismbored Bethune dye amplifiers [22], ${ }^{3}$ the diameters of which are indicated in the diagram. The dye cells are pumped by the second harmonics $(532 \mathrm{~nm})$ of a commercial Nd:YAG laser (Quanta-Ray GCR-5) with a repetition rate of $30 \mathrm{~Hz}$. Therefore, the maximal rate of data acquisition for this system is limited to $30 \mathrm{~Hz}$. In order to synchronize the 80 -

\footnotetext{
'The core assembly codes that interface the program to the frame grabber are provided by Talktronics, Inc..

${ }^{2}$ Typical power output is $400 \mathrm{~mW}$, measured using a Coherent Fieldmaster power meter coupled with a Coherent power meter head model LM- 10.

${ }^{3}$ The Exciton LDS798 dye is used for 800 -nm lines.
} 
$\mathrm{MHz}$ oscillator and the $30-\mathrm{Hz}$ pump laser, a fast-response photodiode ${ }^{1}$ is situated at the back of the high-reflecting end mirror of the oscillator to pick up the residual fs pulses. The output of the photodiode is then fed into a pulse divider circuit (a variant of Schmidt trigger), which triggers both the Nd:YAG flash lamp and a digital delay generator (Stanford Research DG535). DG535 also controls the synchronization among YAG Qswitch, computer data acquisition, and IR-FPA gating (to be discussed later). At this stage, each laser shot has energy of about $7 \mu \mathrm{J}$ but its temporal profile is substantially elongated. $^{2}$ In order to make them suitable for subsequent continuum generation in sapphire windows, ${ }^{3}$ these laser pulses are recompressed with a pair of prisms (comp 1 in the diagram). A $\lambda / 2$ plate (c) is necessary to rotate the beam polarization such that it is at the Brewster angle against the prism surface. After recompression, the temporal FWHM is about $66 \mathrm{fs}$.

In our experimental scheme of UV-Vis pump and IR probe (cf. Figure 1.1), three different colors are required: an $800-\mathrm{nm}$ and a $690-\mathrm{nm}$ beam to generate the $\sim 5 \mu \mathrm{m}$ probe, and a $580-\mathrm{nm}$ beam to generate the $\sim 290-\mathrm{nm}$ pump. As will become clear later, the spectrometer is not limited to these wavelengths. For clarity, however, the following description uses these three colors as examples to demonstrate the working of the dye amplifying chain. Approximately $20 \%$ of the recompressed $800-\mathrm{nm}$ light is further amplified by a $3-\mathrm{mm}$ Bethune cell to give $-19-\mu \mathrm{J}$ pulses with unnoticeable pulse broadening. The remaining $80 \%$ of the $800-\mathrm{nm}$ light is further divided into two arms (50\%:50\%) and focused through a pair of sapphire windows to generate white-light continuum. Note that another critical requirement for a stable continuum generation is a good spatial mode from the seeding light. The white light is amplified by a $1-\mathrm{mm}$ amplification cell ${ }^{4}$ before passing through a bond-pass filter (FWHM $=10 \mathrm{~nm}$ ), which selects the $690-\mathrm{nm}$ (or $580-\mathrm{nm}$ ) portion of the continuum. The amplification of selected

\footnotetext{
${ }^{1}$ EG\&G C30971 series or equivalent that have a response time $<1$ ns at $90-100 \mathrm{~V}$ inversed bias.

${ }^{2}$ Measured using a pyroelectric joule meter (Molectron model J4-05, response $=0.44 \mu \mathrm{J} / \mathrm{mV}$ ).

${ }^{3}$ The typical conditions are E $\sim 1 \mu \mathrm{J} /$ pulse, temporal FWHM $<100$ fs.

${ }^{4}$ The Exciton LDS698 and LDS610 dyes are used for the 690-nm and 580-nm lines, respectively.
} 
wavelengths is completed by another two-stage dye cells to give $\sim 120 \mu \mathrm{J}$ pulses for the $690-\mathrm{nm}$ line (or $\sim 410 \mu \mathrm{J}$ for the $580-\mathrm{nm}$ line). Again, multiple-stage dye amplification broadens the pulse width. Therefore, an additional pair of prisms are used to recompress the 580 -nm line to give $\sim 290$-fs pulses. The $690-\mathrm{nm}$ line does not require recompression because the IR pulse width is determined by the $800-\mathrm{nm}$ line. This scheme allows a broad range of pump and probe wavelengths simply by selecting appropriate dyes and band-pass filters. Optimal dye concentrations and preparation steps are well documented in the laser logs. Auto-correlation plots for the 800,690 , and $680 \mathrm{~nm}$ pulses are displayed in Figure 2.2.

The $580-\mathrm{nm}$ beam is relay-imaged through a translation stage, ${ }^{1}$ which provides the required time delay between pump and probe. The $290-\mathrm{nm}$ pulses for initiating the chemical reaction are generated by frequency-doubling the $580-\mathrm{nm}$ light in a $300-\mu \mathrm{m}$ BBO-II doubling crystal. The typical energy and pulse width at the sample are $\sim 5 \mu \mathrm{J} /$ pulse and $290 \mathrm{fs}$, respectively. The $\sim 5 \mu \mathrm{m}$ probe light is generated by mixing the $800-\mathrm{nm}$ and $690-\mathrm{nm}$ light in a $2-\mathrm{mm}$, type-II $\mathrm{LiIO}_{3}$ crystal. The resulting IR pulses have a temporal FWHM of $\sim 70 \mathrm{fs}$, a pulse energy of $-2 \mu \mathrm{J}$, and a polarization parallel to the optical table. Spectral analyses of the 690, 800, and IR beams are displayed in Figure 2.3. Since we are mostly interested in the population dynamics in the chemical systems, the pump and probe have a polarization angle of $54.7^{\circ}$ (the magic angle). This is achieved by a $\lambda / 2$ plate placed in the $580-\mathrm{nm}$ line, because it is more difficult to manipulate the IR beam. To reduce shot-to-shot noise, ${ }^{2}$ the IR beam is separated (50\%:50\%) into a signal and a reference beam. The signal beam is focused onto a disk of

\footnotetext{
${ }^{1}$ The Klinger translation stage has a stepper drive control whose maximal resolution is $0.25 \mu \mathrm{m}$, and is interfaced with the data acquisition computer via a GPIB control unit, Klinger MC4. Since Klinger has merged with Newport, product support is now provided by Newport. Programming details for this setup can be found in the $\mathrm{C}$ codes that control the data-collection operation, which are included in a separate volume in our laboratory archive.

${ }^{2}$ Laser systems of low-repetition rate such as the system described here tend to have a greater shot-to-shot noise; on the other hand, laser systems of higher repetition rate (hundreds $\mathrm{Hz}$ or $\mathrm{kHz}$ ) tend to have lowfrequency drift. Therefore, the normalization scheme will be different.
} 
diameter $<200 \mu \mathrm{m}$ at the sample. It is important to have a probe focal spot smaller that the pump focal spot to minimize concentration gradient and pronounced transient / thermal lensing from the pump. Since the sample preparation and setup vary in different experiments, they will be described separately in each chapter.

Two detection schemes have been used in the work described in this dissertation. The main difference is the use of a pair of single-element detectors as opposed to a focalplane-array detector. While the latter will be described in more detail in next section, the former single-element scheme is briefly described below. The above-mentioned signal and reference beams are focused into a monochromator (CVI DK-240) with a grating of 150 grove $/ \mathrm{mm}$ and a blazing wavelength of $4 \mu \mathrm{m}$. The configuration of the frequencyresolving instrument determines the spectral resolution of the fs-IR spectrometer. Our configuration has a typical resolution of $\sim 4 \mathrm{~cm}^{-1}$. After passing through the monochromator, the reference and signal beams are respectively focused onto a pair of matched liquid-nitrogen-cooled mercury-cadmium-telluride (MCT) detectors. ${ }^{1}$ The $\mathrm{AC}$ outputs from the on-board pre-amps of the detectors are integrated by boxcar integrators (Stanford Research SR250). Finally, the boxcar outputs are digitized by the existing CAMAC (Computer Automated Measurement And Control) system. The detailed interfacing information can be found in the supplementary volumes of Ph.D. theses of former graduate student, Dr. D. J. Russel and Dr. M. C. Asplund, and will not be repeated here. Noise reduction is achieved by rejecting the digitized data points whose counts are below (or above) certain thresholds. ${ }^{2}$

\footnotetext{
${ }^{1}$ They were acquired from Electro-Optical Systems, Inc., model MCT10-020-E for the detectors and PS1 for the power supply. Under operational conditions, the thermal noise is about $50 \mathrm{mV} / \mathrm{shot}$ at $1-\mathrm{M} \Omega$ impedance.

${ }^{2}$ Current CAMAC is a 11-bit digitizer, which affords a dynamics range of 0-2047 counts. Typical upper and lower bounds are 1800 and 600 , respectively. Because of the limited dynamic range, neutral-density filters in the IR range are required to normalize the reference and signal beam to optimize the experimental condition.
} 


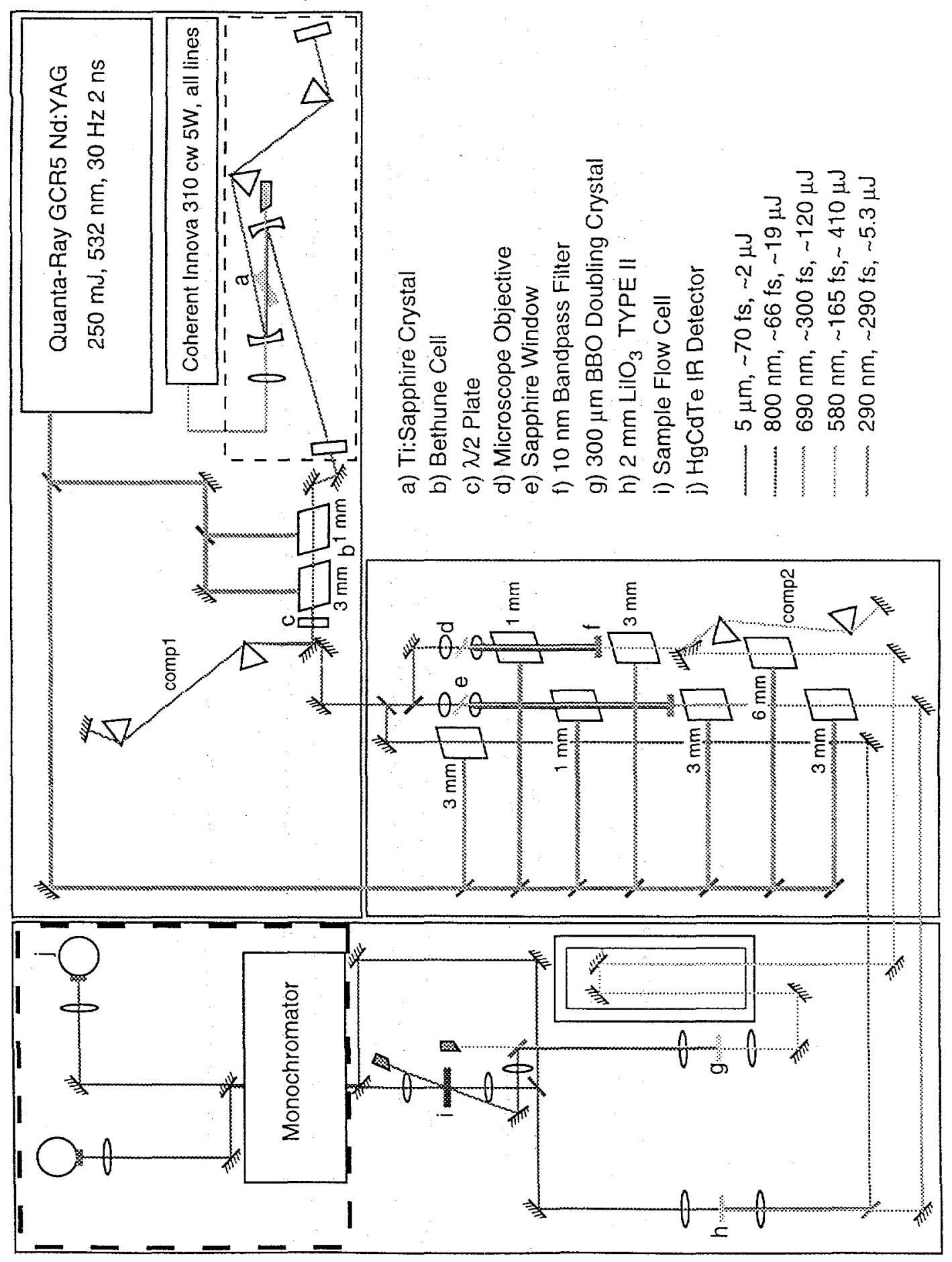

Figure 2.1 Schematics of the experimental setup. 
In spite of its relatively easy setup, the single-element scheme has several drawbacks. For example, it requires typically one or two weeks of continuous run (24 hours) to obtain an IR spectrum in the CO stretching region with a sparse spectral density of $\sim 2.5 \mathrm{~cm}^{-1} /$ point. This spectral density is certainly not enough to resolve overlapping peaks that are bound to appear as the research evolves to include systems that are more complicated. In addition, the long data-taking time makes it almost impossible to have more than one project going at the same time. An IR focal-plane-array (IR-FPA) detector, which affords acquiring a $\sim 70 \mathrm{~cm}^{-1}$ spectrum at one laser shot, improves the situation largely.

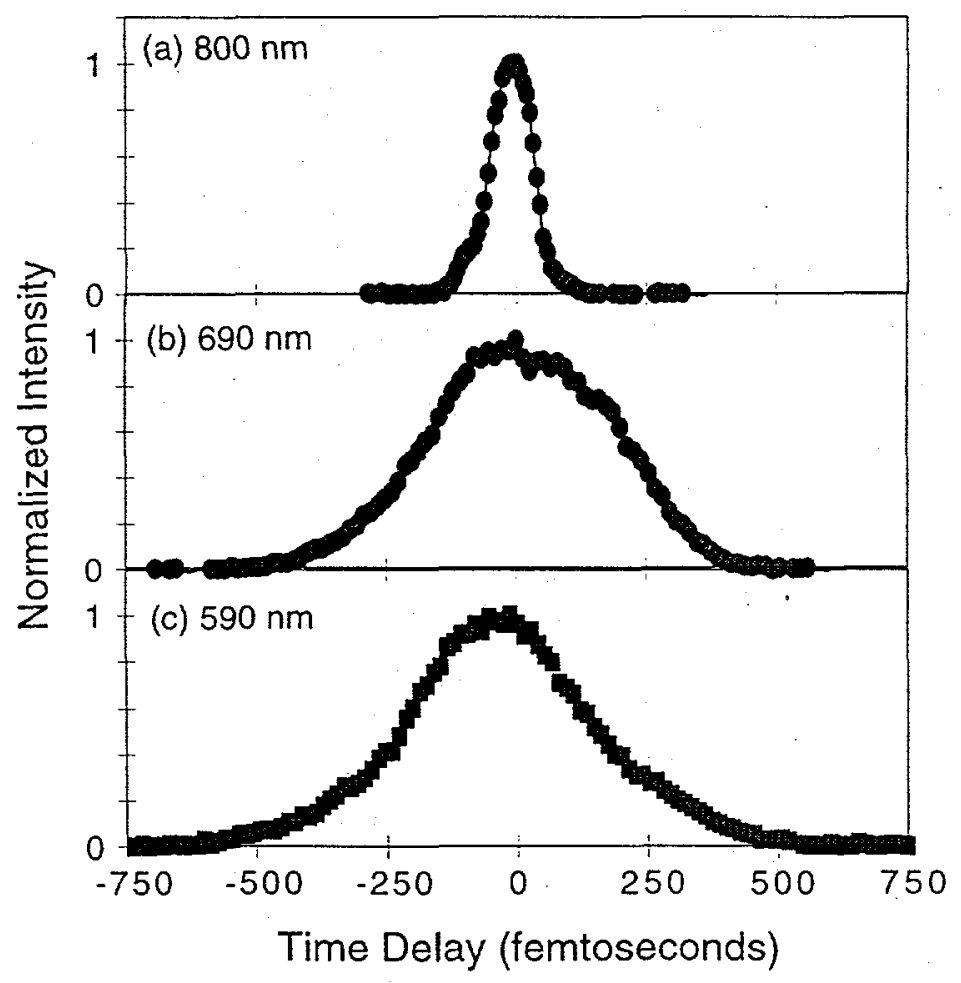

Figure 2.2 Auto-correlation plots of the three laser pulses. 

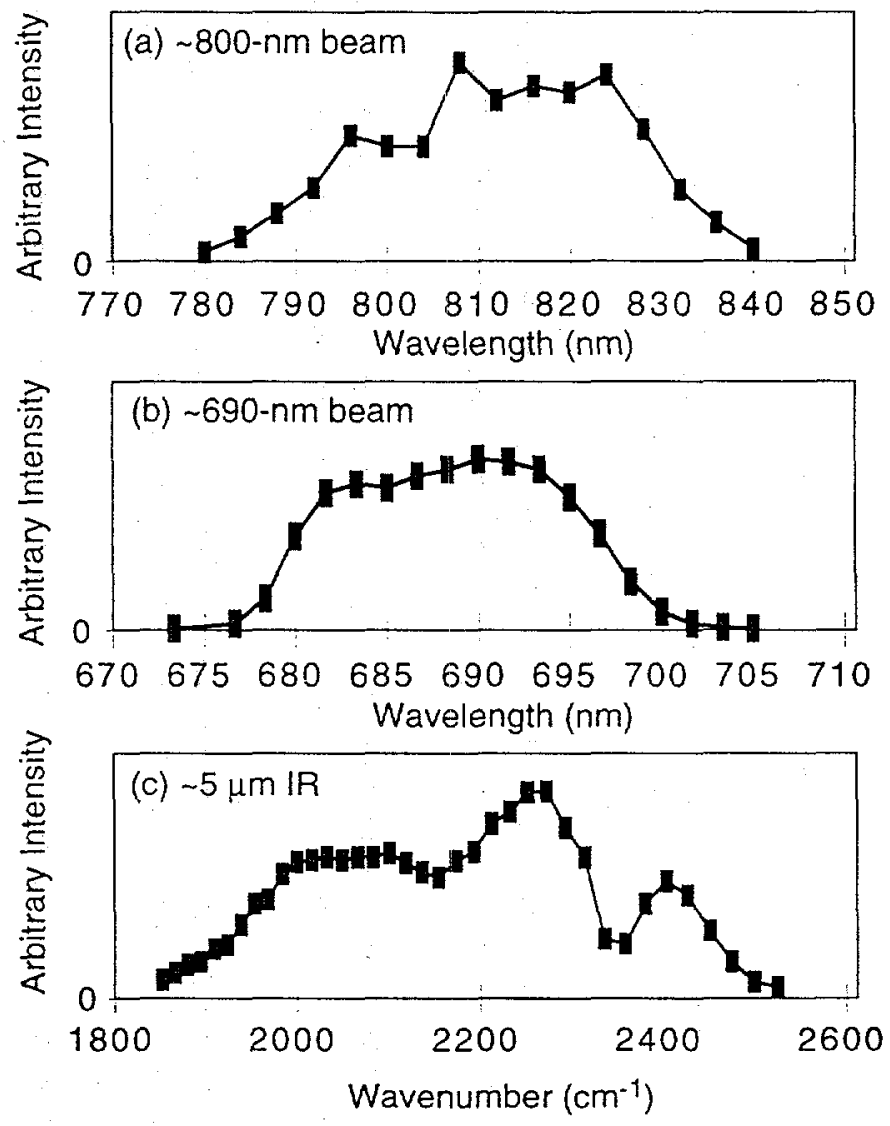

Figure 2.3 Spectral analyses of the ultrafast pulses.

(a) A spectral analysis of the $800-\mathrm{nm}$ beam that is used to generate IR; (b) A spectral analysis of the $690-\mathrm{nm}$ beam that is used to generate IR. Note that in this case, the band profile reflects the characteristics of the band-pass filter used in the dye amplification process; (c) A spectral analysis of the IR generated by mixing the $800-\mathrm{nm}$ and $690-\mathrm{nm}$ beam in a $2-\mathrm{mm} \mathrm{LiIO}_{3}$ nonlinear crystal. This plot illustrates the accessible range for the current setup without changing the nonlinear crystal or the mixing laser wavelengths. The dip at $\sim 2350 \mathrm{~cm}^{-1}$ is believed to result from an absorption band of the $\mathrm{LiIO}_{3}$ crystal. For this particular plot, the phase-matching condition was optimized at each data point. 


\subsection{IR Focal Plane Array (IR-FPA) Detector}

The heart of the IR-FPA detector is an engineer grade, 256×256-element $\mathrm{HgCdTe}$ (MCT) sensor chip of dimension $1.28 \times 1.28 \mathrm{~cm}^{2}$ (or $50 \times 50 \mu \mathrm{m}^{2}$ per pixel). Although the chip can be operated at room temperature, it is kept in contact with a 4-L liquid nitrogen dewar to increase its sensitivity in the IR range. To minimize the shot-to-shot fluctuation and transient heating of the detector, the IR probe pulses are separated into a reference beam and signal beam, and are normalized as the data are being collected. These two beams are focused into an astigmatism-corrected spectrographic monochromator (150 grove/mm, $4.0 \mu \mathrm{m}$ blazed grating) that form two spectrally resolved images on the FPA chip. The two stripes are collected by two windows of $12 \times 200$ pixels, which allow simultaneous normalization of a $\sim 70 \mathrm{~cm}^{-1}$ spectrum. The long-time temperature drift is minimized by normalizing the gain against the FPA readouts from a small region far away from the illuminated area. Rejection of bad laser shots further improves the S/N ratio. With a data acceptance ratio of about 0.5 and a $30-\mathrm{Hz}$ laser repetition rate, it takes about 1 minute to acquire signals on the order of $1 \%$ absorbance change with $10: 1 \mathrm{~S} / \mathrm{N}$ ratio after signal averaging for 1000 valid laser shots. On the other hand, it would take approximately 10 minutes using single-element detectors to obtain a fs-IR spectrum with the same $S / N$ ratio at the same spectral density $\left(200\right.$ points over $\left.\sim 70 \mathrm{~cm}^{-1}\right)$. Therefore, the new FPA detector gives a 10-fold speed boost in taking fs-IR spectra. The time required for kinetic data acquisition is also greatly reduced. The kinetics of a certain chemical species can be obtained by summing the desired IR band across 10's of spectral points. Assuming that every pixel is independent of each other and that they have similar intrinsic noise level, this summation scheme gives effectively more than 10-fold speed increase in acquiring kinetic data. The increased spectral data density also allows a more detailed analysis of spectra that contain overlapping bands, as will be explained in the C$\mathrm{Cl}$ bond activation chapter. 


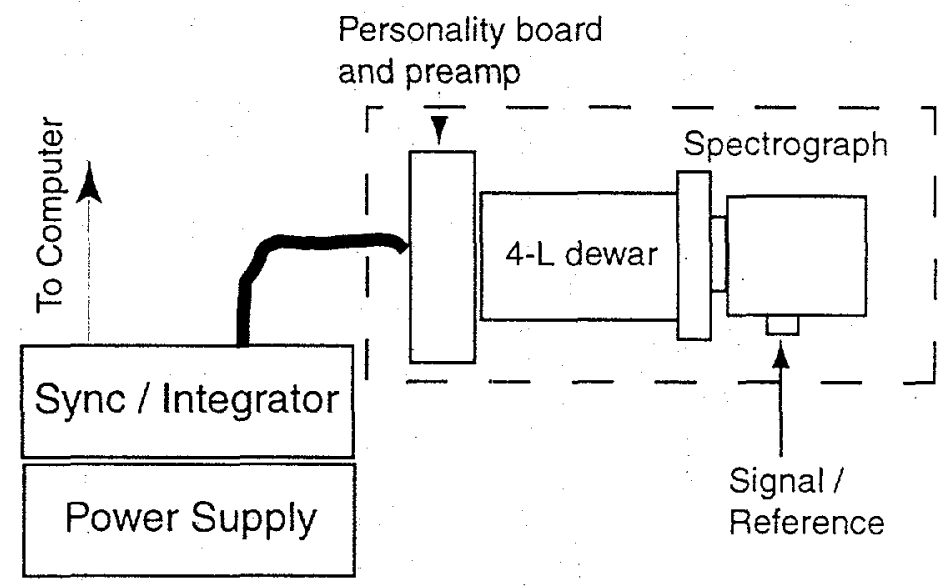

Figure 2.4 Schematics of the IR-array setup.

Setup. To replace the single-element detector with the FPA, one simply exchanges the original detection system including the monochromator (enclosed by the dashed line in Figure 2.1) with the FPA setup. Figure 2.4 shows a block diagram of the FPA setup that fits into the existing laser system. Both the dewar assembly and the spectrograph sit on separate tilt-adjustable plates to compensate for mismatch in mechanical mounting. Once the initial optimization is complete, it is a simple matter to remove / reinstall the dewar assembly for maintenance. A nitrogen line is connected to the spectrograph to keep moisture from condensing on the interior or the front window of the detector.

The details of the modifications made to the Sync-integrator unit have been annotated in the original schematics ${ }^{1}$ and described in the laboratory notebook. 'Briefly, the analog amplifying chain has been boosted from 3 to 114 . Together with the gain provided by preamp $(1.1 \mathrm{x})$, the total gain is about 125 . It was found that the amplifying chains are very sensitive to temperature. Therefore, a warm-up time of $20 \mathrm{~min}$ is required for the system to reach thermal equilibrium. After setting up the analog gain, one has to balance the odd- and even-channels. This has been achieved by adjusting the $T_{1}$ to $T_{5}$ variable resisters on the chain-amplifiers. The analog-to-digital conversion

\footnotetext{
${ }^{1}$ Schematics title: High-Speed Signal Chain; Revision H; Schematics date: May 30, 1997.
} 
(ADC) board has a 16-bit resolution, which affords a dynamic range of 0-65535 for each pixel. In practice, only half of the available dynamic range is utilized. The origin of the background noise is believed to be due to the quality of the chip (engineer grade) and incomplete discharge of the capacitor. The latter can be alleviated by continuously discarding a few data points before accepting one and has been encoded into the software. The integration time also affects the background noise. It can be changed by flipping the dip-switches on the $\mathrm{J} 7$ board. $^{2}$ The current setting is $1.6 \mu \mathrm{s}$. The optimal integration time may vary with system. In the event that the opamps employed in the above setup require replacement, it would be necessary to go through the procedure outlined above to calibrate the system. Other settings including the bias potentials are not expected to change for the same sensor chip; therefore, one can leave them as they are at the optimized settings.

Software. Additional drivers for our shutter and translation stage are encoded into the interface program ${ }^{3}$ that came with the IR-FPA. Efforts have been made on the software level to minimize the signal fluctuation. The source codes are archived in the computer archives of our laboratory. Modification of the program should be straightforward for persons with minimal C-programming knowledge. Note that it can only be successfully compiled with "Microsoft $\mathrm{C}++7.0$ " because some of the low-level drivers were written with specific dependencies on the 7.0 libraries.

\subsection{Frequency Chirping at the Vicinity of a Strongly-Absorbing IR Band}

Most of the theoretical framework of UV-Vis pump, IR probe spectroscopy has been established in the mid-90's [23, 24]; therefore, they will not be recounted here.

\footnotetext{
${ }^{1}$ H. Yang's notebook volume IV, dated Mar. 6, 1997 to May 17, 1998.

${ }^{2}$ A table of dip switch settings and corresponding integration time can be found on page 122 in the previously mentioned notebook.

${ }^{3}$ Part of the interface program is credited to Dr. Tom Dougherty while he was at NIST.
} 
However, there were two transient features that puzzled us in our initial studies of dipolar solvation of LDS-750 dye (styrine 7) in nitrile solutions. With the publication of a related study by Kovalenko et. al. [25], the wavelength-independent transient that exhibits a longer decay time in longer-chain nitrile solutions appears to be the transient absorption of a low-lying electronic excited state. The other unassigned transient feature can now be attributed to pump induced chirping described below.

The intense pump field induced change in the refraction index, or transient grating, has been intensively investigated in the past decade [26]. The majority of the studies were conducted in the off-resonant optical region where appropriate approximations can be made to simplify data analysis; sometimes it is possible to arrive at an analytical expression. However, in the infrared region, a $\sim 100$-fs IR pulse that spans $\sim 150 \mathrm{~cm}^{-1}$ in frequency may cover several vibrational transitions such that many approximations that are taken for granted in the analysis of optical experiments are no longer valid. This section explains one aspect of nonlinear optical-pump, IR-probe spectroscopy that has not been clearly discussed in the literature. The complication arises when the pump field is not in resonance with the sample, be it pure liquid or solution, but the probe field covers at least one vibrational transition. Therefore, additional analyses seem necessary to understand the transient signals that appear in such experiments.

\subsubsection{Theory}

In the following, it is assumed that the system consists of only pure liquid (the medium) that has an intense infrared absorption overlapping with the probe field. Extension to solutions of more than one component and/or to molecular media with multiple IR transitions should be straightforward. One starts by writing down the expression for frequency-resolved probe signal $I_{\text {probe }}\left(\omega_{3} t_{d e l}\right)$ in a pump-probe configuration [27], 
Equation 2.1

$$
I_{\text {probe }}\left(\omega, t_{d e l}\right)=\int_{-\infty}^{+\infty} d t r_{\text {probe }}^{\prime}\left[t, \omega+\Delta \omega\left(t, t_{\text {del }}\right)\right],
$$

where $t_{d e l}$ is the pump-probe delay and $\Delta \omega\left(t, t_{d e l}\right)$ is the new frequency component introduced by the pump. The $I^{\prime}$ probe is the probe field envelope centered at $\omega_{0}$ and can be well represented by a Gaussian function,

Equation 2.2

$$
I_{\text {probe }}^{\prime}(t, \omega)=\exp \left(-\left(t / \sigma_{\text {probe }}\right)^{2}\right) \exp \left(-\left(\frac{\omega-\omega_{0}}{\sigma_{\omega 0}}\right)^{2}\right)
$$

In the above expression, $\sigma_{\text {probe }}$ and $\sigma_{\omega 0}$ are the pulse width and the spectral width of the probe.

The intense pump laser beam (off resonance) interacts with the medium to induce a change in the index of refraction, $\delta n$,

Equation 2.3

$$
\delta n(t)=n_{2}\left(\omega_{\text {pump }}, \omega_{\text {probe }}\right) I_{\text {pump }}(t),
$$

where, to a very good approximation, the pump field envelope can be represented by a Gaussian function,

Equation 2.4 $\quad I_{\text {pump }}(t)=\exp \left(-\left(t / \sigma_{\text {pump }}\right)^{2}\right)$

and $n_{2}\left(\omega_{\text {pump }}, \omega_{\text {probe }}\right)$ is the nonlinear index of refraction which depends on both the pump and probe frequencies [28]. For a time-varying pump field, the induced third-order response introduces a time-dependent phase shift,

Equation 2.5

$$
\delta \phi(t)=\frac{2 \pi L}{\lambda_{\text {probe }}} \delta n(t),
$$

where $L$ is the sample thickness and $\lambda_{\text {probe }}$ is the wavelength of the probe. When the probe pulse passes through the medium, the time-dependent phase modulation caused by the pump field introduces new frequency components into the probe field (frequency chirping): 
Equation 2.6 $\Delta \omega=\frac{\partial}{\partial t} \delta \phi(t)$

The phase shift can be separated into a vibronic component $\delta \phi_{e}$ and a nuclear component (molecular diffusion and rotation) $\delta \phi_{n}$ as $\delta \phi(t)=\delta \phi_{e}(t)+\delta \phi_{n}(t)$ [29-33], where

Equation 2.7 $\quad \delta \phi_{e}=\frac{2 \pi L n_{2, e}}{\lambda_{\text {probe }}} I_{\text {pump }}(t)$

and

Equation 2.8

$$
\delta \phi_{n}=-\beta \Theta(t) e^{-\left(t / T_{1}\right)}\left[\left(\frac{1}{T_{1}}+\frac{1}{T_{2}}\right) e^{-\left(t / T_{2}\right)}-\frac{1}{T_{1}}\right],
$$

where $n_{2, e}$ is the nonlinear index of refraction for electronic transition, $\beta$ is a scaling factor, $\Theta(t)$ is the Heaviside function. The $T_{1}$ and $T_{2}$ are the decay and rise times, respectively. They describe phenomenologically the relaxation of the medium after impulsive perturbation. The best way to quantify the above parameters is to perform an optical Kerr effect (OKE) experiment in the frequency region of interest since they are related to internal properties of the medium. In the optical region, it is also possible to carry out $a b$ initio calculations to determine the value of $n_{2, e}$ [34]. When the probe frequency is in or close to resonance with the vibrational transition $\omega_{i b}$ of the solvent molecules, to a good approximation, the vibronic contribution is greatly enhanced by a resonance factor, $1 /\left(\omega_{\text {probe }}-\omega_{v i b}+i \Gamma_{v i b}\right)$, ${ }^{1}$ where $\Gamma_{v i b}$ is the damping factor (or dephasing rate) for the vibrational transition [35]. Although the current experimental setup is not susceptible to the nuclear term, ${ }^{2}$ the complete expression is explained below nonetheless.

\footnotetext{
${ }^{1}$ This is the leading term, omitting scaling factors, of a perturbative vibronic expansion of the nonlinear susceptibility of the material. See [35] for details.

${ }^{2}$ With our current setup, an IR polarization analyzer or up-conversion will be needed to resolve the nuclear part of the signai.
} 
If one assumes that the frequency shift $\Delta \omega$ is small, Equation 2.2 can be expanded in a Taylor series to give an approximate analytical expression for the signal [27],

Equation 2.9

$$
\Delta O D\left(t_{d e l}, \omega\right)=-\log \left\{1+\frac{4}{\sqrt{\pi}} \frac{\left(\omega-\omega_{0}\right)}{\sigma_{\omega 0}^{2} \sigma_{\text {probe }}^{3}}\left[A_{e}\left(t_{d e l}\right)+A_{n}\left(t_{d e l}\right)\right]\right\rangle .
$$

The electronic contribution is ${ }^{1}$

Equation 2.10

$$
A_{e}\left(t_{d e l}\right)=4 L \omega_{0} n_{2, e} \sigma_{\text {pump }} \sigma_{\text {probe }}\left(\frac{\pi}{\sigma_{\text {pump }}^{2} \sigma_{\text {probe }}^{2}}\right)^{3 / 2} t_{\text {del }} \exp \left[-\left(\frac{t_{\text {del }}^{2}}{\sigma_{\text {pump }}^{2}+\sigma_{\text {probe }}^{2}}\right),\right.
$$

and the nuclear contribution is

\section{Equation 2.11}

$$
\begin{aligned}
& A_{n}\left(t_{\text {del }}\right)=\beta \exp \left\{-E_{1}\left[\frac{d_{1}}{2} \sigma_{\text {probe }} \sqrt{\pi} \operatorname{erfc}\left(\frac{t_{d e l}-d_{1}}{\sigma_{\text {probe }}}\right)+\frac{\sigma_{\text {probe }}^{2}}{2} e^{-\left(\frac{t_{d e l}-d_{1}}{\sigma_{\text {probe }}}\right)^{2}}\right]\right\} \\
& -\beta \exp \left\{-E_{2}\left[\frac{d_{2}}{2} \sigma_{\text {probe }} \sqrt{\pi} \operatorname{erfc}\left(\frac{t_{\text {del }}-d_{2}}{\sigma_{\text {probe }}}\right)+\frac{\sigma_{\text {probe }}^{2}}{2} e^{\left.-\left(\frac{t_{d e l}-d_{2}}{\sigma_{\text {probe }}}\right)^{2}\right]}\right],\right.
\end{aligned}
$$

where

$$
\begin{aligned}
& d_{1}=-\frac{\sigma_{\text {probe }}^{2}}{2 T_{1}} \\
& d_{2}=-\frac{\sigma_{\text {probe }}^{2}}{2}\left(\frac{1}{T_{1}}+\frac{1}{T_{2}}\right), \\
& E_{1}=\frac{t_{\text {del }}}{T_{1}}-\left(\frac{\sigma_{\text {probe }}}{2 T_{1}}\right)^{2}
\end{aligned}
$$




$$
E_{1}=t_{d e l}\left(\frac{1}{T_{1}}+\frac{1}{T_{2}}\right)-\left[\frac{\sigma_{\text {probe }}}{2}\left(\frac{1}{T_{1}}+\frac{1}{T_{2}}\right)\right]^{2}
$$

\subsubsection{Comparison with Experiments}

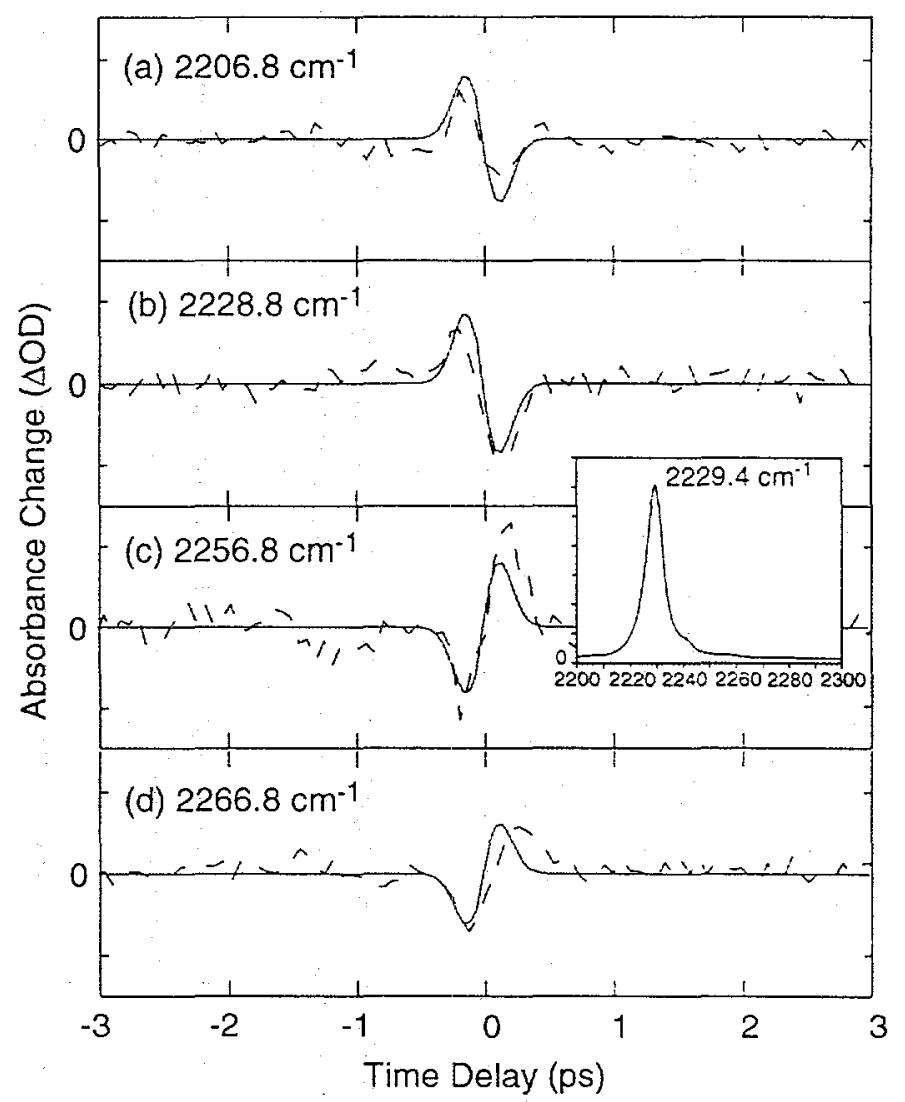

Figure 2.5 Comparison of experiments with theory for pump induced IR chirping.

Figure 2.5 compares the transient signals (long dashed lines, pumping at $580 \mathrm{~nm}$ ) of neat benzonitrile to the theory (solid lines). In the experiment, the IR beam was optimized at $2000 \mathrm{~cm}^{-1}$. Therefore, the calculation assumes that the IR has a central frequency of $2000 \mathrm{~cm}^{-1}$, and a spectral bandwidth of $150 \mathrm{~cm}^{-1}$. The damping constant

${ }^{1}$ There is a mistake in Eq. (11) of Ref. [27], probably a typo: 
$\Gamma_{v i b} \approx 3.85 \mathrm{~cm}^{-1}$ is estimated from the width of the $\mathrm{CN}$ stretch $\left(2229.4 \mathrm{~cm}^{-1}\right)$ of the static FTIR spectrum shown in the insert. The same scaling factor is used for all theoretical lines. As displayed in the figure, the transients change sign as the pump-probe delay passes $t_{0}$ because the probe field is phase-modulated by the pump (Equation 2.6). The direction of the modulation is determined by the time-derivative of the pump field, which changes sign before and after $t_{0}$. The transients also change sign at either side of the $\mathrm{CN}$ band, as a direct result of the near-resonant term, $1 /\left(\omega_{\text {probe }}-\omega_{v i b}+i \Gamma_{v i b}\right)$.

\subsection{The Vibrational Stark Effect}

Consider the vibrational motion of a diatomic molecule, say $\mathrm{CO}$, the frequency of which is determined by the distribution of the electron density. In a static picture, the electron distribution-or equivalently the force constant of the oscillator-changes when the molecule is subjected to an external field. For polyatomic molecules, the external field may also break the symmetry such that new vibrational bands may appear. Such a change of vibrational frequency due to an external field, or vibrational Stark effect, have been observed in zeolites [36], adsorbates on metal surfaces [37-41], and biological samples [42]. These results suggest the potential utilization of vibrational Stark effect as a probe for the dynamics of local environment. This section discusses some basic aspects of the vibrational Stark effect (VSE) and methods of calculating the frequency shift.

\subsubsection{An Analytical Model}

A theoretical model for VSE has been formulated by Lambert in the 80's [38, 43]. This section outlines the derivation to illustrate the coupling between the external field and the frequency shift. In the following discussion, the vibrational coordinate of an 
oscillator, say $\mathrm{CO}$, is expressed in normal coordinate $Q$ in the field-free vibrational Hamiltonian,

Equation 2.12 $\quad H=\frac{1}{2 \mu} p_{Q}^{2}+\frac{1}{2} \mu \omega^{2} Q^{2}$,

where $\mu$ is the reduced mass, $p_{Q}$ the conjugate momentum, and $\omega$ the vibrational frequency. Qualitatively, the application of a static external electric field causes redistribution of the electron density and that of the nuclei such that the equilibrium positions of the nuclei are different from those without an external field. The result is a change in the vibrational frequency.

To find the frequency shift, one first writes the vibrational potential $V$ that is under a static electric field $E$ in the form of perturbative expansion:

Equation 2.13 $\quad V(Q, E)=\sum_{j, k} a_{j k} Q^{j} E^{k}$

Recasting the above equation in an expansion with respect to the new equilibrium position $Q_{0}(E)=-\frac{a_{11}}{2 a_{20}} E$ when there is an external field $E$,

$$
\text { Equation 2.14 } \quad V(\bar{Q})=c_{0}+c_{2} \bar{Q}^{2}+c_{3} \bar{Q}^{3}+\cdots,
$$

where

Equation 2.15 $\bar{Q}=Q-Q_{0}(E)$

Expressions of $c_{n}$ in terms of $a_{i j}$ are found by comparing the Taylor expansion of Equation 2.13 to Equation 2.14. One finds that

Equation 2.16

$$
c_{n}=\left.\frac{1}{n !} \frac{\partial^{n} V(Q, E)}{\partial Q^{n}}\right|_{Q=Q_{0}}=a_{n 0}+a_{n+1.0}(n+1) Q
$$

The $v=0 \rightarrow 1$ transition frequency derived from Equation 2.14 is 
Equation 2.17 $\quad v_{01}=\frac{1}{2 \pi}\left(\frac{2 c_{2}}{\mu}\right)^{1 / 2}+$ higher order terms .

Hence, the frequency shift with respect to the external field, the vibrational Stark tuning rate, is

Equation 2.18

$$
\delta_{v E}^{(n)}=\left.\frac{\partial^{n} v_{01}}{\partial E^{n}}\right|_{E=0}
$$

$$
\delta_{v E}^{(1)}=\frac{1}{2 \pi}\left(\frac{2 a_{20}}{\mu}\right)^{1 / 2}\left(\frac{2 a_{20} a_{21}-3 a_{30} a_{11}}{4 a_{20}^{2}}\right)
$$

In the above expression, the coefficients $a_{20}$ and $a_{30}$ are related to the field-free molecular vibrational frequency and anharmonicity, which can be obtained from gasphase spectroscopy; whereas estimation of the other two parameters $a_{21}$ and $a_{11}$ require additional experiment data under external fields. Using the above expression, Lambert was able to estimate the contribution of higher-order terms to the vibrational tuning rate and found that the second- and third-order terms contribute less than $5 / 1000$ of the total magnitude.

\subsubsection{First-Principle Calculations}

Although the above derivation provides a satisfactory physical insight into the vibrational Stark effect, it is very likely that one cannot find the appropriate parameters for the molecules under study. Alternatively, one can also calculate the vibrational tuning rate using $a b$ initio methods [44-52]. It has been found that both the basis set and electron correlation influence the absolute magnitude of the calculated vibrational tuning rate. Calculations of the vibrational tuning rate for $\mathrm{CO}$ at different levels of electron correlation and basis sets are carried out in order to find and calibrate an $a b$ initio approach suitable for larger molecules such as nitriles. Figure 2.6 shows a representative plot of the scaled calculated frequencies against external fields (open circles) and the 
polynomial fit (solid line) that determines the vibrational tuning rate $\delta_{v E}$. Selected results are listed in Table 2.1, ${ }^{1}$ which shows that the use of the 6-311G(d,p) basis set at the Hartree-Fock level produces the best result comparing to the experimental value ($5.09 \pm 1.0) \times 10^{-7} \mathrm{~cm}^{-1}(\mathrm{~V} / \mathrm{cm})^{-1}[38]$. Therefore, the same methodology is applied to calculating $\delta_{V E}$ for the $\mathrm{CN}$ stretch of acetonitrile and benzonitrile. Figure 2.7 shows the results for acetonitrile. The directions of the external field and the molecular dipole are indicated in the insert. It is evident from the plots that higher-order terms of $\delta_{v E}$ become important at greater field strength. In general, an acetonitrile molecule tends to align its dipole with the field (positive direction on the plots), thus shifting its vibrational frequency to the red while augmenting its vibrational intensity. Similar conclusions can be made to benzonitrile, results shown in Figure 2.8.

With these results, one can estimate the frequency shift that may result from a dipole change of, say, 10 Debye of a probe molecule. If one assumes a continuous dielectric medium of acetonitrile and estimates the frequency shift of acetonitrile at $4 \AA$ away from the probe molecule, one obtains only $0.8-\mathrm{cm}^{-1}$ shift. However, if one assumes vacuum between the probe molecule and an acetonitrile, the result becomes $27 \mathrm{~cm}^{-1}$ ! A more reliable calculation would be to perform a molecular dynamics simulation, to be described in the next section. Nonetheless, the above calculations suggest that it is possible to utilize the VSE as a probe for the dynamics of the local environment. Potential applications include probing the local response to a charge-transfer reaction and following the backbone dynamics of a macromolecule.

\footnotetext{
${ }^{1}$ The calculations were carried using G.AMESS-US, Ref. [53].
} 
Table 2.1 Selected results for characterization of different $a b$ initio approaches to calculating the vibrational tuning rate.

\begin{tabular}{ll}
\hline Method & $\delta_{v E} \mathrm{~cm}^{-1}(\mathrm{~V} / \mathrm{cm})^{-1}$ \\
\hline HF/6-311G(d,p) & $-6.17 \times 10^{-7}$ \\
MP2/6-311G(d,p) ${ }^{\mathrm{a}}$ & $-1.94 \times 10^{-7}$ \\
$\mathrm{HF} / 6-311 \mathrm{G}(\mathrm{d}, \mathrm{p})^{\mathrm{b}}$ & $-5.59 \times 10^{-7}$ \\
HF/6-31G(d,p) & $-5.82 \times 10^{-7}$ \\
\hline A scaling factor of 0.9496 is applied for this calculation [54]. \\
${ }^{\mathrm{a}}$ A scaling factor of 0.9051 is applied for this calculation [54]. \\
A scaling factor of 0.8992 is applied for this calculation [54].
\end{tabular}

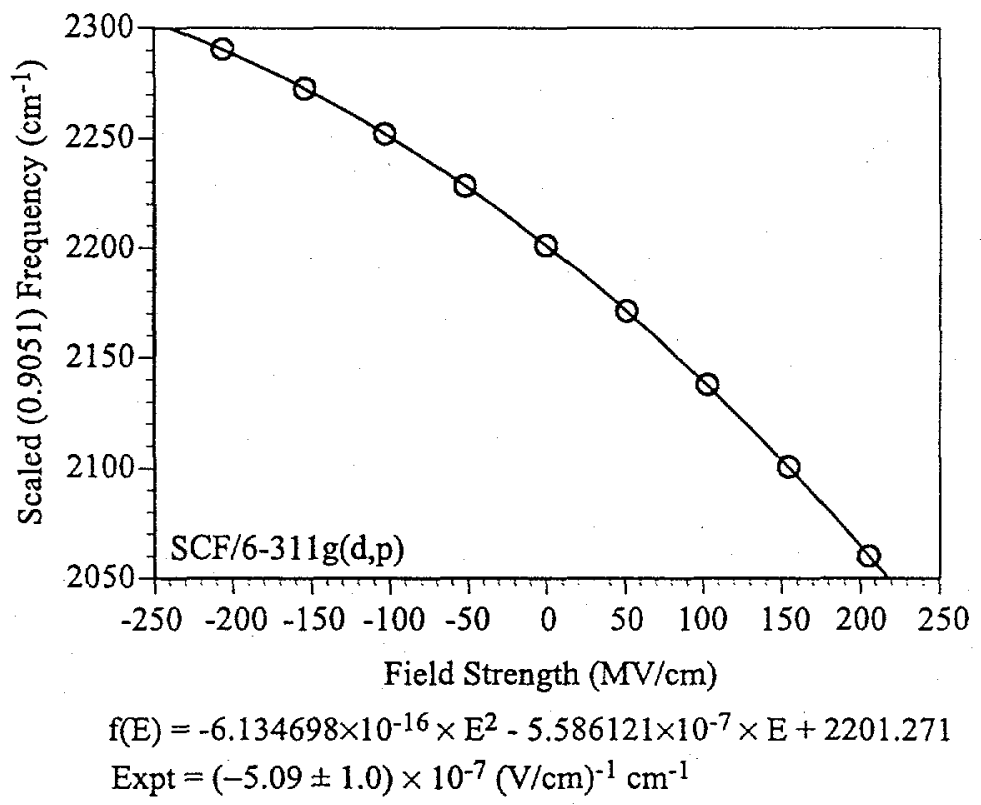

Figure 2.6 Evaluation of $a b$ initio vibrational Stark effect for $\mathrm{CO}$. 

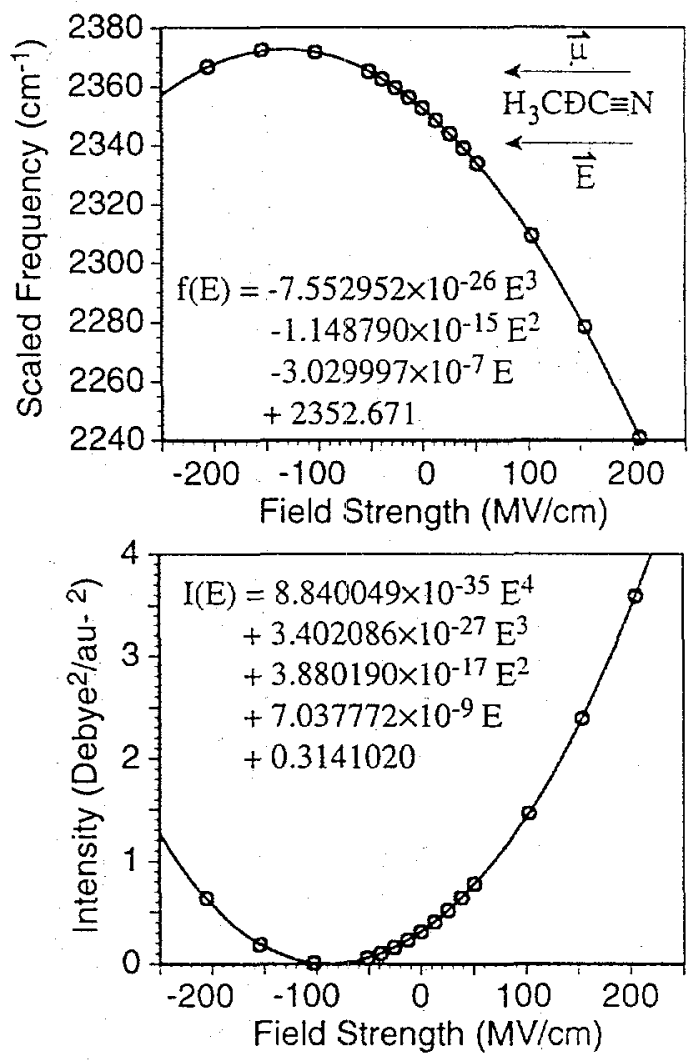

Figure 2.7 Evaluation of $a b$ initio vibrational Stark effect for $\mathrm{CH}_{3} \mathrm{CN}$. 

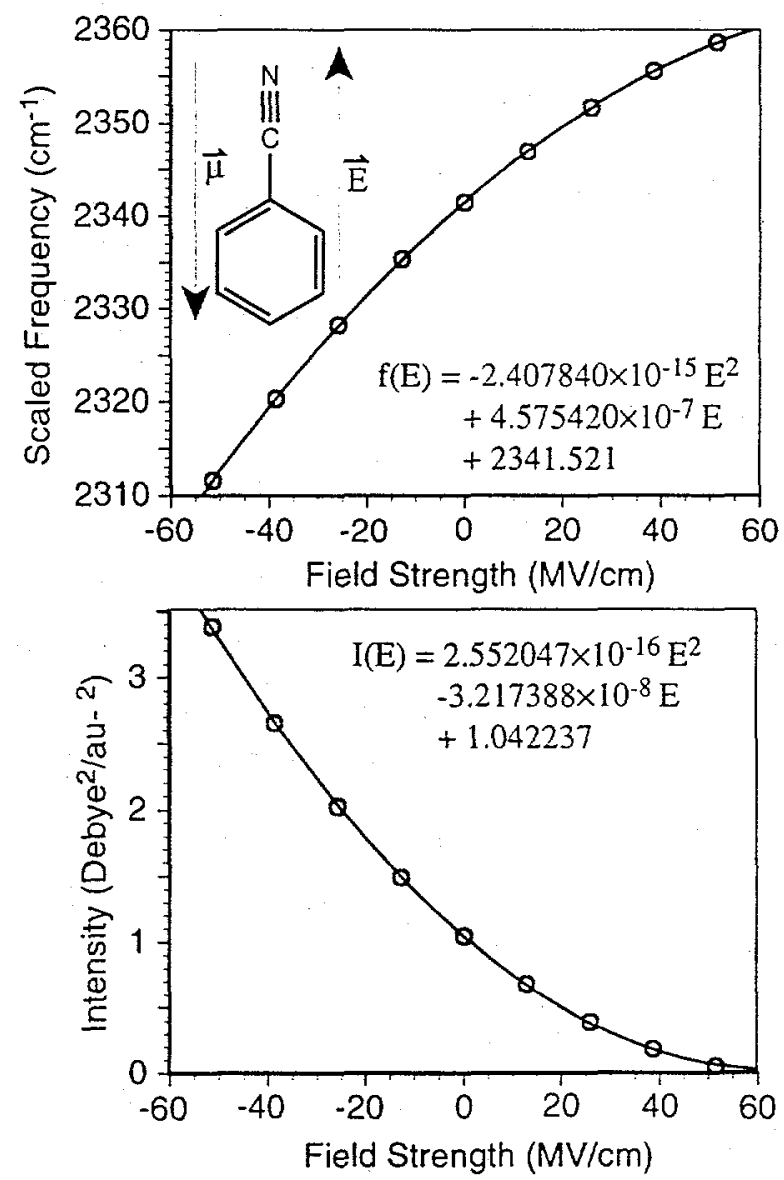

Figure 2.8 Evaluation of $a b$ initio vibrational Stark effect for $\mathrm{C}_{6} \mathrm{H}_{5} \mathrm{CN}$. 


\subsection{Classical Molecular Dynamics Simulation}

The physical picture for the dynamics of a liquid-phase chemical reaction cannot be complete without including the surrounding solvent molecules. The previous two sections have covered theoretical bases for the design and interpretation of femtosecond IR experiments to directly monitor such solvent dynamics. However, the experimental results are essentially ensemble averages of the observables. A direct means of connecting the microscopic molecular motion to the experimental observable is through statistic analysis of molecular dynamics (MD) simulations. This section summarizes the development and implementation of a MD engine that is suitable for those purposes.

\subsubsection{Potential Models}

The potential model is a modified version of the three-site SPC model of Edwards et. al. [55] that was parameterized to experimental thermodynamic properties. This model, as originally devised, assumes a rigid molecular structure of the acetonitrile molecule. The current implementation adopts the same intermolecular interaction form, on top of which an intramolecular potential is grafted for simulations of flexible acetonitrile.

The intermolecular interactions consist of Lennard-Jones potentials and Coulombic interactions between different atomic sites. The form of the intermolecular potential function is given by

Equation 2.19

$$
U^{(2)}\left(\mathbf{r}_{1}, \mathbf{r}_{2}\right)=\sum_{i=1}^{N-1} \sum_{j=i+1}^{N} \sum_{a=1}^{n_{a}} \sum_{b=1}^{n_{a}}\left\{4 \varepsilon_{a, b}\left[\left(\frac{\sigma_{a, b}}{r_{i a, j b}}\right)^{6}-\left(\frac{\sigma_{a, b}}{r_{i a, j b}}\right)^{12}\right]+\frac{1}{4 \pi \varepsilon_{0}} \frac{q_{a} q_{b}}{r_{i a, j b}}+C_{i a, j b}\right\}
$$


where $N$ is the number of molecules being simulated and $n_{a}$ is the number of atoms in a molecule. The indices $i$ and $j$ refer to the molecules, whereas the subscripts $a$ and $b$ represent the methyl (Me) group, the carbon or the nitrogen atom sites. The $q_{a}$ and $q_{b}$ are the fractional charges in units of $e, \varepsilon_{0}$ is the permittivity of free space and $C_{i a . j b}$ is the constant for correcting the Lennard-Jones cut-off:

Equation 2.20

$$
C_{i a, j b}=\left\{\begin{array}{cc}
0 & \text { when } r_{i a, j b} \leq R_{c u t}=2.5 \sigma_{a . b} \\
4 \varepsilon_{a, b}\left[\left(\frac{\sigma_{a, b}}{R_{c u t}}\right)^{6}-\left(\frac{\sigma_{a, b}}{R_{c u t}}\right)^{12}\right] & \text { when } r_{i a, j b}>R_{c u t}
\end{array}\right.
$$

The cross interaction parameters are determined by the combining rules

Equation 2.21 $\quad \varepsilon_{a, b}=\left(\varepsilon_{a, a} \varepsilon_{b, b}\right)^{3 / 2}, \sigma_{a, b}=\frac{1}{2}\left(\sigma_{a, a}+\sigma_{b, b}\right)$

The numerical values of the parameters are given in Table 2.1.

Table 2.2 Intermolecular parameters for acetonitrile MD simulation.

\begin{tabular}{llll}
\hline Parameter & $\mathrm{CH}_{3}$ & $\mathrm{C}$ & $\mathrm{N}$ \\
\hline$q\left(\mathrm{e}^{-}\right)$ & 0.269 & 0.129 & -0.398 \\
$\sigma(\AA)$ & 3.6 & 3.4 & 3.3 \\
$\varepsilon(\mathrm{K})$ & 191 & 50 & 50 \\
\hline$r\left(\mathrm{H}_{3} \mathrm{C}-\mathrm{C}\right)=1.458 \AA, r(\mathrm{C}-\mathrm{N})=1.157 \AA$.
\end{tabular}

The harmonic intramolecular potential is a quadratic form in $\Delta r_{1}, \Delta r_{2}$, and $\Delta \theta$ :

Equation 2.22

$$
V_{H M}=\frac{1}{2} k_{1} \Delta r_{1}^{2}+\frac{1}{2} k_{2} \Delta r_{2}^{2}+\frac{1}{2} k_{\theta} \Delta \theta^{2}
$$

where $\Delta \theta$ is the change in Me-C-N angle and $\Delta r_{1}, \Delta r_{2}$ are the stretches in the Me-C and $\mathrm{C}-\mathrm{N}$ bond lengths, respectively. The force constants $k_{1}, k_{2}$, and $k_{\theta}$ are calculated from gas-phase spectroscopic data [56] by the standard "FG" matrix decomposition method [57]. Parameters used for intramolecular potential are: $k_{1}=0.3066551978, k_{2}=$ 1.0695597563 , and $k_{\theta}=0.0096949221$. 


\subsubsection{Simulation Details}

All bulk acetonitrile simulations are performed with 108, 256, or 500 acetonitrile molecules in a periodically replicated cubic box of length $L$ such that the density is at its ambient value, $0.78745 \mathrm{~g} \mathrm{~cm}^{-3}$ [58]. The long-range Coulombic forces are treated by standard Ewald sums method [59] with a total number of $221 \mathrm{k}$-vectors. The minimum image convention is used for Lennard-Jones interactions with truncation length $L / 2$. A "weak-coupling" constant temperature algorithm [60] with a coupling constant of 0.4 ps yields an average temperature of $\sim 300 \mathrm{~K}$. Care has been taken to ensure equipartition of energies among all degrees of freedom. A separate temperature scaling scheme [61] for center-of-mass motions and internal degrees of freedom (vibrational and rotational) is utilized for simulations of flexible $\mathrm{CH}_{3} \mathrm{CN}$. As Wallqvist and Teleman have discussed in a simulation of flexible water [61], it takes more than 100 ps to equipartition the energy among all modes for a harmonic model when the Coulombic forces are treated without the Ewald summation. However, the relaxation time reduces to $\sim 100$ ps when the Ewald summation is used. With their separate temperature-scaling scheme, the equilibrating time further reduces to within 4 ps. In this work, the relaxation time is also found to be within 4 ps.

For simulations of flexible acetonitrile, the equations of motion are integrated using the reversible reference system propagator algorithm (r-RESPA) developed by Tuckerman, Berne, and Martyna [62]. In the present implementation, the intramolecular forces are considered as "hard forces" and the corresponding motions are integrated at time steps of $0.75 \mathrm{fs}$. The "soft forces" consisting of the Lennard-Jones and Coulombic interactions are integrated at time steps of $3.75 \mathrm{fs}$. The r-RESPA method provides a computational saving of a factor of 4 in this application. The equations of motion for rigid acetonitrile are solved with the Singer-Taylor's method [63] and integrated using the Verlet leapfrog algorithm [59]. The time step for rigid acetonitrile simulations is 10 
fs. The energy fluctuations for both the flexible model and the rigid model are found to be within $0.5 \%$.

For each run, the system is prepared in an $\alpha$-f.c.c. configuration, allowed to equilibrate for $\sim 100 \mathrm{ps}$, and then run out up to $\sim 1 \mathrm{~ns}$. The thermodynamic data are then sampled at every 10 ps. These equilibrated configurations serve as the starting configurations for later nonequilibrium simulations. The dynamic properties are calculated from a $\sim 20$-ps run after a 10-ps equilibration time starting with an equilibrated configuration. All MD codes are written in C and run on UNIX workstations.

\subsubsection{Results}

Figure 2.9 shows representative diagnostic results from equilibrium runs of the flexible acetonitrile. The velocity-velocity autocorrelation functions of each site shown in Figure 2.9a demonstrate the available frequency components for such a model liquid. The four distinct spectral features are the nuclear motion, the $\mathrm{CH}_{3}-\mathrm{C}-\mathrm{N}$ bending, the $\mathrm{CH}_{3}-\mathrm{C}$ stretching, and the $\mathrm{C}-\mathrm{N}$ stretching, respectively. The center-of-mass particleparticle autocorrelation function shown in Figure $2.9 \mathrm{~b}$ clearly demonstrates the solvent shell structure around a tagged solvent molecule. Such a long-range structure disappears when the tagged solute is charged or has a permanent dipole moment as will be demonstrated in the next two figures. 

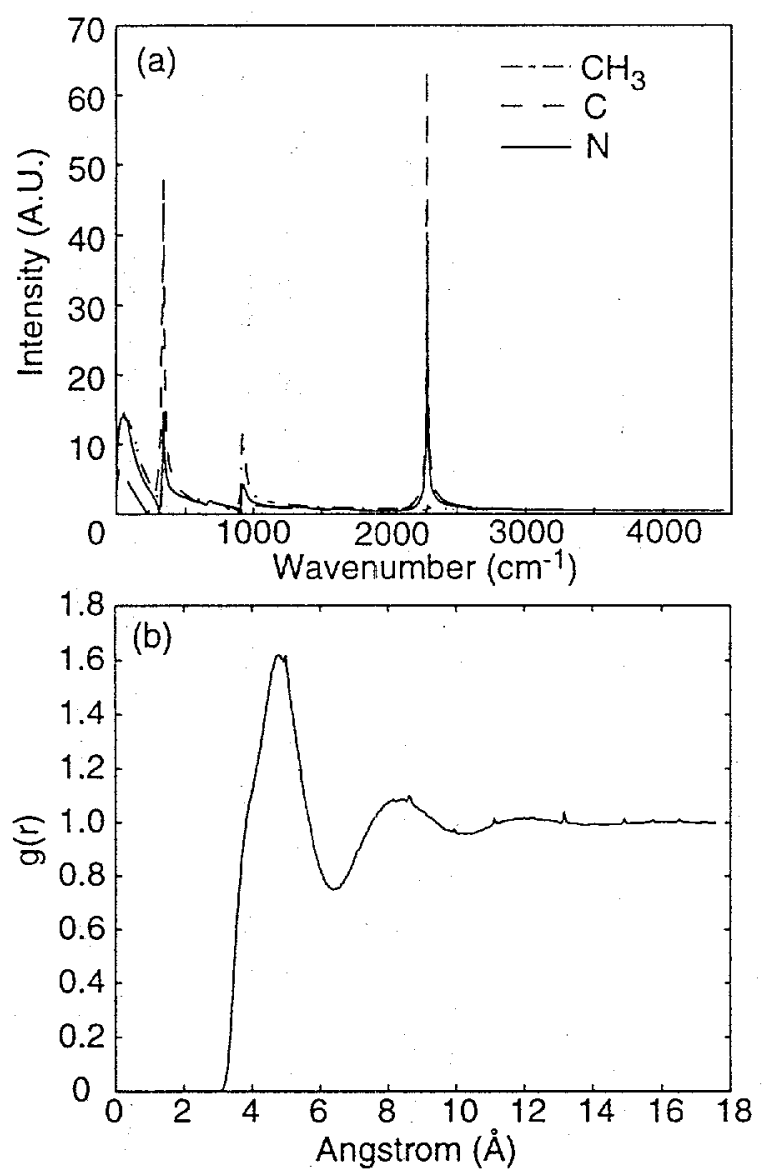

Figure 2.9 Characterization of a MD simulation engine for $\mathrm{CH}_{3} \mathrm{CN}$.

(a) A 1-ns run of 108 flexible acetonitrile molecules with the model potential described in the text; (b) A $\sim 1$-ns run of 500 flexible acetonitrile molecules. 

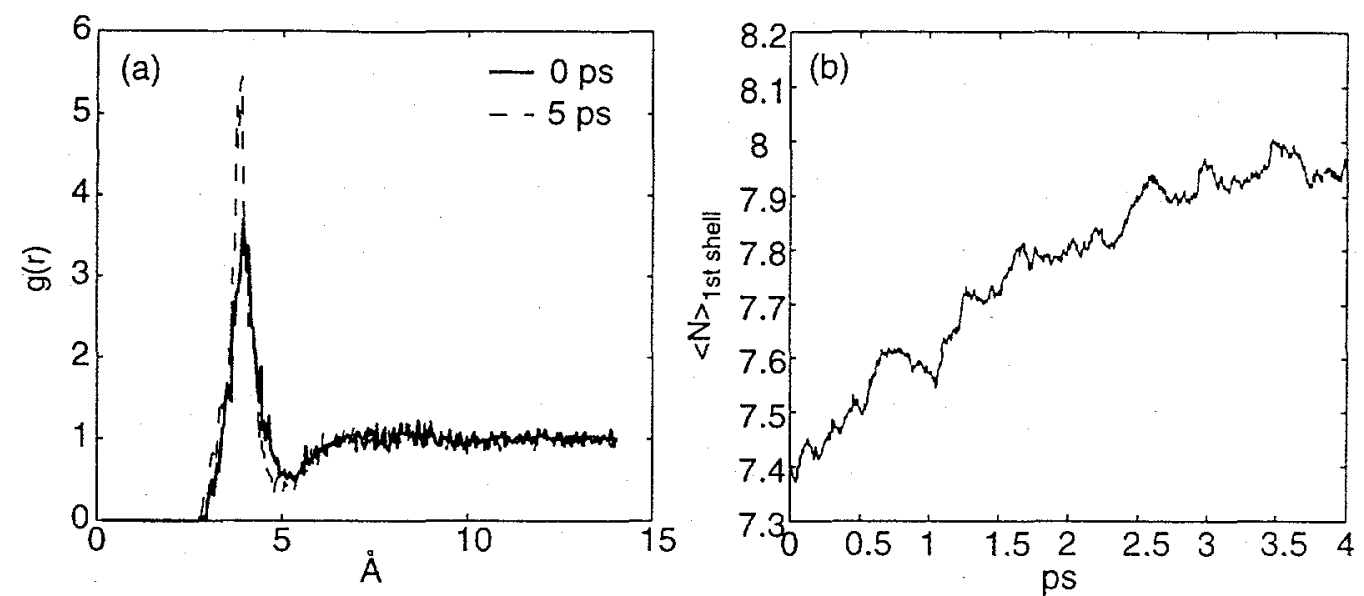

- Average over 348 traces

- Solute $6.55 \rightarrow 15$ Debye

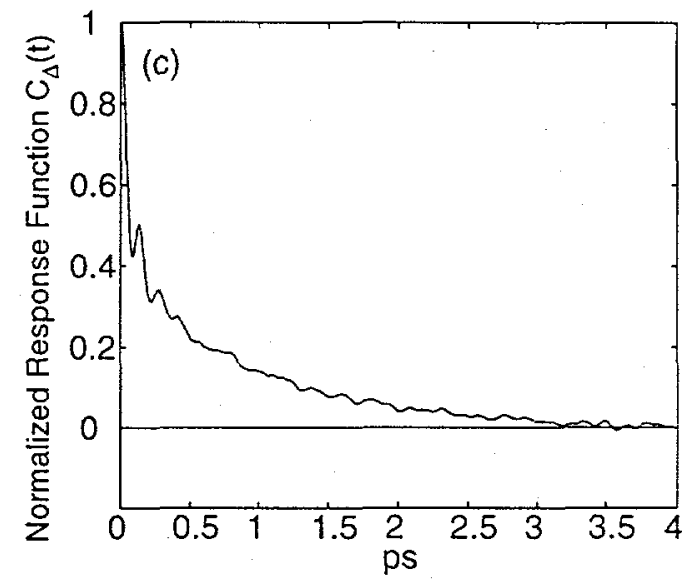

Figure 2.10 Non-equilibrium MD simulation of dipole solvation for a sudden dipole change of 8.45 Debye.

Figure 2.10 shows selected results from nonequilibrium runs of a solute that is $6-\AA$ in diameter. The solute changes its dipole moment from 6.55 to 15 Debye at $t=0$, following which the solvent dynamics are monitored for 4 ps. Figure $2.10 \mathrm{a}$ displays the radial distribution function of solvent molecules with respect to the solute. One sees that when the solute is charged or has a permanent dipole, only the first solvent shell has a clear structure; the solute and solvent molecules maintain their spatial correlation in a much shorter range than a neutral solute. Comparing the distribution of $t=0 \mathrm{ps}$ with that of $t=5 \mathrm{ps}$, one sees that the increased solute dipole attracts more solvent molecules around it. Such translational solvation dynamics can be quantified by monitoring the 
mean population $\langle\mathrm{N}\rangle$ of the solvent molecules in the first solvation shell shown in Figure 2.10b. One sees that $\langle N\rangle$ increases gradually from $\sim 7.4$ to $\sim 7.9$ on the simulation time scale. Figure $2.10 \mathrm{c}$ shows the normalized response function as a function of time. The initial fast solvation $(>50 \%$ ) has been attributed to solvent librational motion, which retains its memory in this case for hundreds of femtoseconds as the small oscillations indicate. Next figure compares the solvation dynamics of an identical solute, the dipole change of which is of identical magnitude but opposite sign.
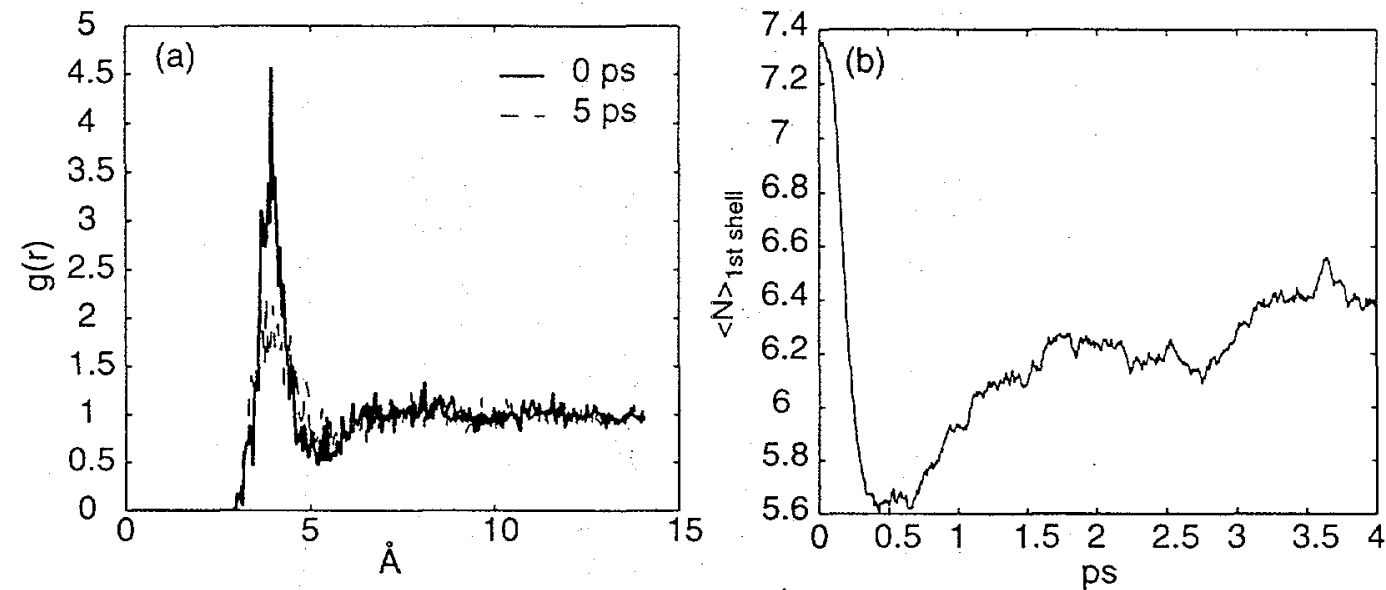

- Average over 246 traces

- Solute $6.55 \rightarrow-1.9$ Debye

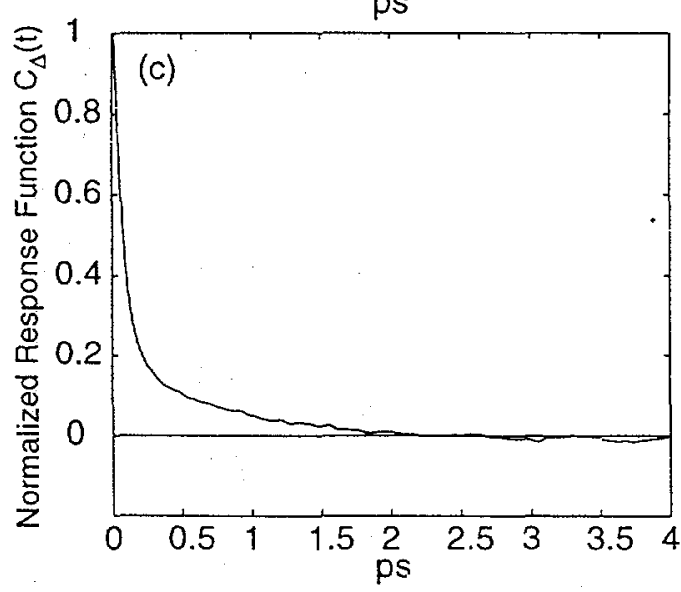

Figure 2.11 Non-equilibrium MD simulation of dipole solvation for a sudden dipole change of -8.45 Debye. 
One sees that in this case the mean-population of the first-solvation shell decreases when the solute changes its sign and magnitude. Figure $2.11 b$ shows that $\langle\bar{l}\rangle$ firstly decreases from $\sim 7.4$ to $\sim 5.6$ within 500 fs, then recovers to $\sim 6.4$ at 4 -ps after dipole change. This can be understood as follows. Before $t=0$, the solute has a greater dipole moment such that acetonitrile is better aligned with the solute dipole. When the solute dipole suddenly reverses its direction and reduces the magnitude, the solvent molecules no longer experience an attracting force from the solute, but a strong repulsing field. This results in the initial ballistic motion of the solvent to move away from the energetically unfavorable solute within 500 fs. At a longer time scale, the solvent molecules rearrange to accommodate themselves for the new field the system gradually reaches a new equilibrium. It is interesting to note that the energy correlation function plotted in Figure 2.11c exhibits a smooth, monotonic decay ( $>80 \%$ within $500 \mathrm{fs}$ ) as opposed to small oscillations observed in the previous case. Both Figure 2.11b and Figure 2.11c are consistent with the picture that, for this case, the initial ballistic motion of the first solvent shell is greatly "frustrated" by the outer-shell solvent molecules."

\subsection{Conclusion}

This chapter has covered several topics that may be essential for further development of studying liquid-phase reaction dynamics. In particular, the roles the solvent molecules partake in a complicated chemical reaction remain unclear and await further elucidation. It is hoped that some of the concepts discussed in this chapter will eventually contribute to future studies of the fascinating field of chemical reaction dynamics.

\footnotetext{
${ }^{1}$ During the course of the MD simulation studies, it was found that the solute charge density (charge/size) plays an important in the dynamics of dipolar solvation. In particular, the degree of solvent "frustration" varies with solute charge density. These observations are in line with a very recent paper by Tran and Schwartz [64], who studied effects of solute size in aqueous solutions.
} 


\section{C-H BOND ACtivation BY $\eta^{3}-\mathrm{TP}^{*} \mathrm{RH}(\mathrm{CO})_{2}$}

The natural abundance of alkanes makes them an ideal feedstock for organic synthesis. The first step towards converting an alkane molecule into other synthetically useful compounds is to cleave or activate the strong $\mathrm{C}-\mathrm{H}$ bond (bond energy $\sim 100$ $\mathrm{kcal} / \mathrm{mol}$ ). Consequently, the chemistry needed to selectively activate a $\mathrm{C}-\mathrm{H}$ bond has been vigorously pursued in the past decade [65-69]. Advancements in this area include the development of homogeneous intermolecular $\mathrm{C}-\mathrm{H}$ bond activation by organometallic compounds $[70,71]$. They offer the possibility to tailor a specific reaction utilizing welldeveloped synthetic strategies. The rational design of such a reaction relies significantly on knowledge of both the detailed reaction mechanism and the nature of the reaction intermediates. Previous mechanistic studies of photochemical $\mathrm{C}-\mathrm{H}$ bond activation have focused on the $\eta^{5}-\mathrm{Cp}{ }^{*} \mathrm{M}(\mathrm{CO})_{2}$ ( $\left.\mathrm{M}=\mathrm{Ir}, \mathrm{Rh}\right)$ compounds, which, unfortunately, have relatively low quantum yields [72]. Instead, the present work employs $\eta^{3}-\mathrm{Tp}{ }^{*} \mathrm{Rh}(\mathrm{CO})_{2}$ $\left(\mathrm{Tp}^{*}=\mathrm{HB}-\mathrm{Pz}_{3}{ }_{3}, \mathrm{Pz}^{*}=3,5\right.$-dimethylpyrazolyl, structure shown in Figure 3.1), which has been shown to photochemically activate alkane $\mathrm{C}-\mathrm{H}$ bonds to form the final product $\eta^{3}-\mathrm{Tp}{ }^{*} \mathrm{Rh}(\mathrm{CO})(\mathrm{H})(\mathrm{R})(\mathrm{R}=$ alkyl) with $\sim 30 \%$ quantum yields [73-76].

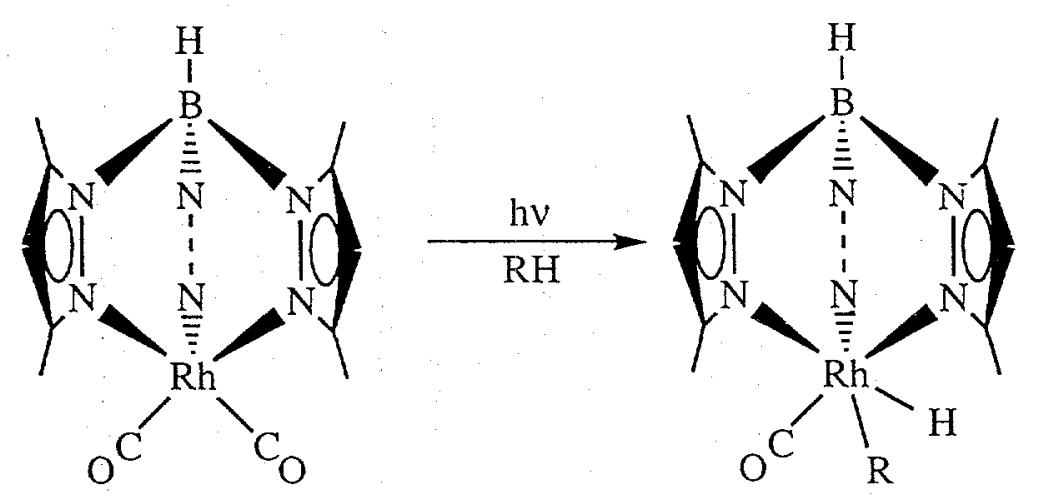

Figure $3.1 \mathrm{C}-\mathrm{H}$ bond activation by $\eta^{3}-\mathrm{Tp}{ }^{*} \mathrm{Rh}(\mathrm{CO})$. 


\section{I The Dynamics of Reaction Intermediates-Vibrational Relaxation and Molecular Morphology Change}

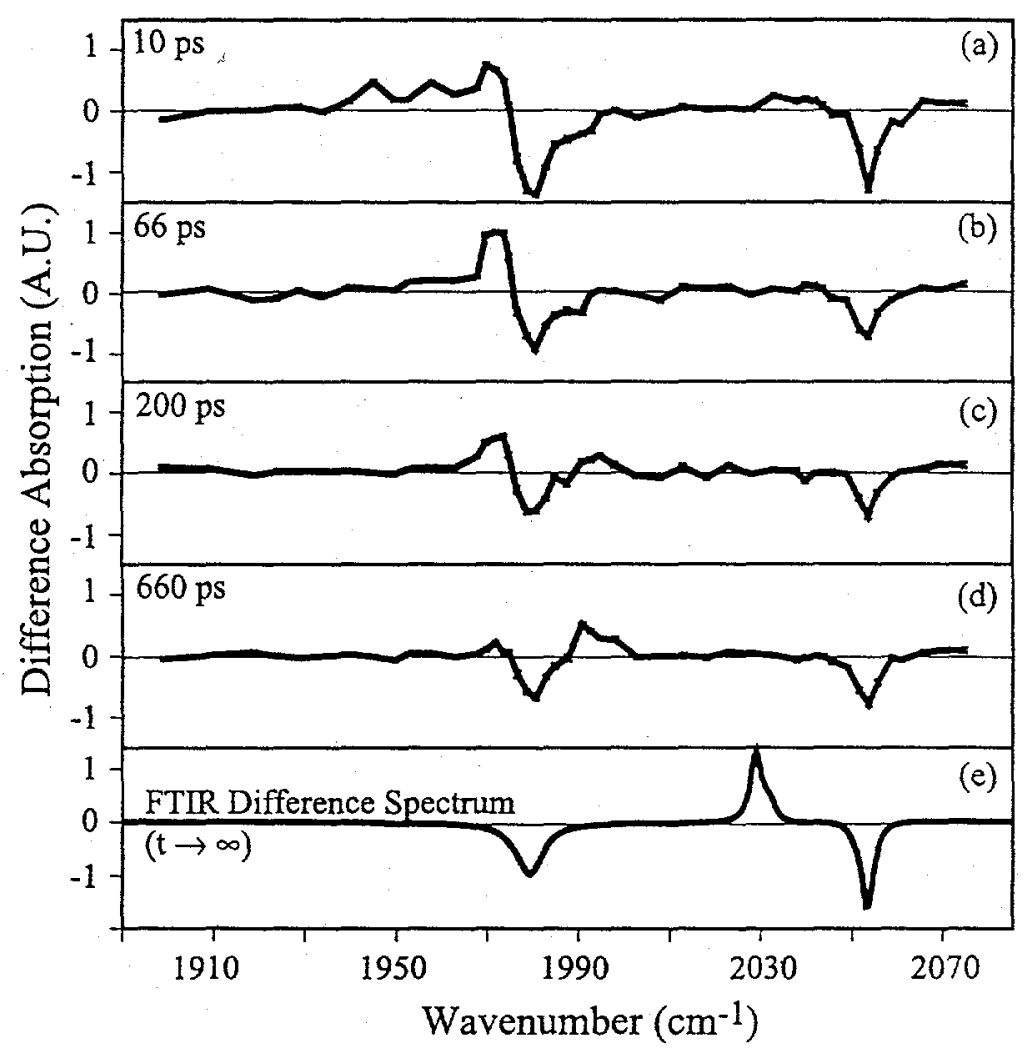

Figure 3.2 Ultrafast IR spectra of $\eta^{3}-\mathrm{Tp} * \mathrm{Rh}(\mathrm{CO})_{2}$ in alkane solution following $295-\mathrm{nm}$ excitation.

Transient difference spectra in the CO stretching region for $\eta^{3}-\mathrm{Tp} * \mathrm{Rh}(\mathrm{CO})_{2}$ in roomtemperature alkane solution at various time delays following $295-\mathrm{nm}$ photolysis. Panel (e) is an FTIR difference spectrum before and after 308 -nm photolysis. A broad, wavelength independent background signal from $\mathrm{CaF}_{2}$ windows has been subtracted.

Photolysis of a CO ligand from the parent compound initiates the reaction. The resultant $\eta^{3}-\mathrm{Tp}{ }^{*} \mathrm{Rh}(\mathrm{CO})$ complex has a coordinatively vacant site that is quickly occupied by a solvent molecule. The timescale for such a solvation process is generally on the order of a few ps in room-temperature liquids [77]. The solvated $\eta^{3}-\mathrm{Tp}^{*} \mathrm{Rh}(\mathrm{CO})(\mathrm{S})(\mathrm{S}=$ alkane) complex exhibits a single CO stretching band $\left(v_{\mathrm{CO}}\right)$ at $1972 \mathrm{~cm}^{-1}$ (the $\mathrm{v}=0 \rightarrow 1$ band) as shown in Figure 3.2a. For this particular system the large excess energy ( 40 
$50 \mathrm{kcal} / \mathrm{mol}$ ) deposited by the UV photon creates a non-Boltzman population distribution such that higher vibrational levels may also be occupied to the extent that they become observable. As a result, the $v=1 \rightarrow 2$ and $v=2 \rightarrow 3 v_{C O}$ transitions appear at 1958 and $1945 \mathrm{~cm}^{-1}$, respectively. The two hot bands gradually decay away while the $v=0$ band $\left(1972 \mathrm{~cm}^{-1}\right)$ rises as the system approaches thermal equilibrium. The time scale for such intramolecular relaxation (IVR) is measured by monitoring the population dynamics of the $\mathrm{v}=0$ state. The $1972-\mathrm{cm}^{-1}$ band shows a fast rise of $\sim 23 \mathrm{ps}$, attributed to the above IVR process, and a slower decay of $\sim 200$ ps (Figure 3.3a). The $\sim 200$-ps decay indicates that the $\eta^{3}-\mathrm{Tp}{ }^{*} \mathrm{Rh}(\mathrm{CO})(\mathrm{S})$ solvate reacts to form another species on this time scale. Indeed, another peak appears at $1990 \mathrm{~cm}^{-1}$ at later time delays (Figure 3.2c and Figure 3.2d). The correlation between $\eta^{3}-T p^{*} R h(C O)(S)$ and the $1990-\mathrm{cm}^{-1}$ species is established by the $\sim 200$-ps rise of the $1990-\mathrm{cm}^{-1}$ band (Figure 3.3b) [16].

The $1990-\mathrm{cm}^{-1}$ band is attributed to an $\eta^{2}-\mathrm{Tp} * \mathrm{Rh}(\mathrm{CO})(\mathrm{S})$ solvate where one of the three chelating pyrazolyl ligands detaches itself from the Rh center. To verify this assignment the fs-IR spectra following UV photolysis of $\eta^{3}-\mathrm{Tp}{ }^{*} \mathrm{Rh}(\mathrm{CO})_{2}$ are compared to those of an analogous compound $\eta^{2}-\mathrm{Bp}{ }^{*} \mathrm{Rh}(\mathrm{CO})_{2}\left(\mathrm{Bp}^{*}=\mathrm{H}_{2} \mathrm{~B}-\mathrm{Pz}_{2}{ }_{2}\right.$, structure shown in Figure 3.4). Photolysis of $\eta^{2}-\mathrm{Bp}{ }^{*} \mathrm{Rh}(\mathrm{CO})_{2}$ in alkane solutions results in the $\eta^{2}-\mathrm{Bp}{ }^{*} \mathrm{Rh}(\mathrm{CO})(\mathrm{S})$ solvate that also exhibits a single $\mathrm{CO}$ stretching band at $\sim 1990 \mathrm{~cm}^{-1}$, thereby providing experimental evidence for the assignment [15]. Theoretically, Zaric and Hall computed the reaction using $\mathrm{CH}_{4}$ to model the alkane solvent [78]. Their DFT calculations show that the $\eta^{2}-\mathrm{TpRh}(\mathrm{CO})\left(\mathrm{CH}_{4}\right)\left(\mathrm{Tp}=\mathrm{HB}-\mathrm{Pz}_{3}, \mathrm{Pz}=\right.$ pyrazolyl $)$ complex is energetically more stable than the $\eta^{3}-\mathrm{TpRh}(\mathrm{CO})\left(\mathrm{CH}_{4}\right)$ complex by $7.7 \mathrm{kcal} / \mathrm{mol}$. In addition, the DFT frequency of the model $\eta^{3}$ complex is found $22-\mathrm{cm}^{-1}$ higher than that of the $\eta^{3}$ complex. The calculated frequency shift in the model systems is consistent with the observed $18-\mathrm{cm}^{-1}$ blue shift from $\eta^{3}-\mathrm{Tp}{ }^{*} \mathrm{Rh}(\mathrm{CO})(\mathrm{S})\left(1972 \mathrm{~cm}^{-1}\right)$ to $\eta^{2}-\mathrm{Tp}{ }^{*} \mathrm{Rh}(\mathrm{CO})(\mathrm{S})$ $\left(1990 \mathrm{~cm}^{-1}\right)$. On the basis of the above-discussed evidence, it is concluded that the 1990$\mathrm{cm}^{-1}$ intermediate is assigned as $\eta^{3}-\mathrm{Tp}{ }^{*} \mathrm{Rh}(\mathrm{CO})(\mathrm{S})$. Therefore, the observed 200-ps time 
constant measures the free-energy barrier $\Delta \mathrm{G}^{\dot{亠}} \approx 4.1 \mathrm{kcal} / \mathrm{mol}$ for the $\eta^{3}$-to- $\eta^{2}$ isomerization.'

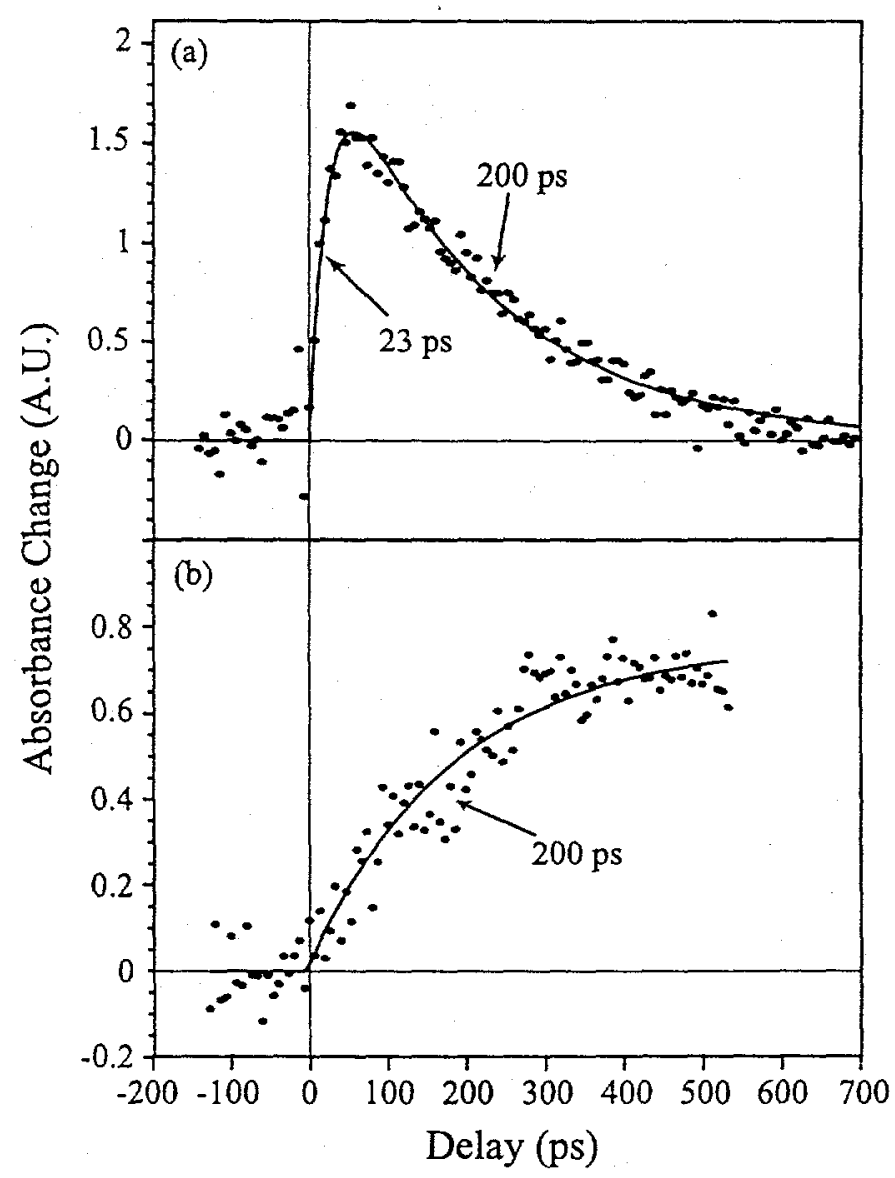

Figure 3.3 Ultrafast kinetics of $\eta^{3}-\mathrm{Tp} * \mathrm{Rh}(\mathrm{CO})_{2}$ in alkane solution.

Ultrafast kinetics (dots) of $\eta^{3}-\mathrm{Tp} * \mathrm{Rh}(\mathrm{CO})_{2}$ in room-temperature alkane solution after 295-nm photolysis at (a) $1972 \mathrm{~cm}^{-1}$, the $\mathrm{CO}$ stretch of the solvated $\eta^{3}-\mathrm{Tp} * \mathrm{Rh}(\mathrm{CO})$ (alkane) intermediate; and (b) $1990 \mathrm{~cm}^{-1}$, the $\mathrm{CO}$ stretch of the armdetached $\eta^{2}-\mathrm{Tp} * \mathrm{Rh}(\mathrm{CO})($ alkane) intermediate.

\footnotetext{
${ }^{1}$ The energy barrier is estimated using a simple transition-state theory expression: $k=1 / \tau=\frac{k_{B} T}{h} \exp (-\Delta G / R T)$.
} 


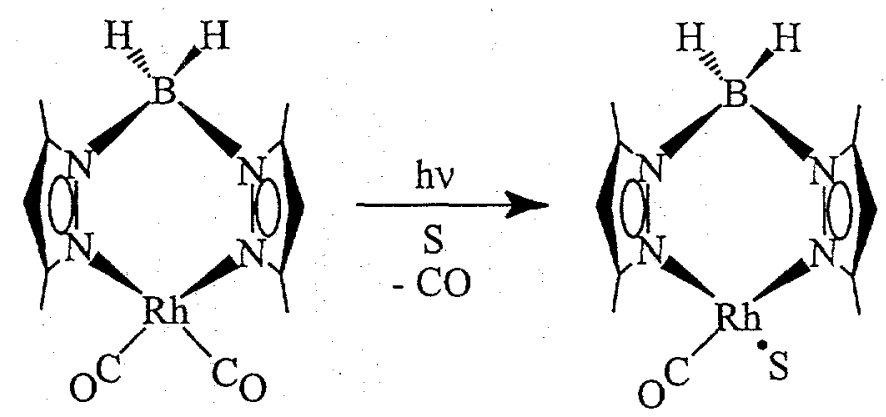

Figure 3.4 Photodissociation of $\eta^{2}-\mathrm{Bp} * \mathrm{Rh}(\mathrm{CO})_{2}$.

\subsection{The Activation Barrier-the Bond-Breaking Step}

The fact that the final product $\eta^{3}-\mathrm{Tp} * \mathrm{Rh}(\mathrm{CO})(\mathrm{H})(\mathrm{R})$ does not appear on the ultrafast time scale $(<1 \mathrm{~ns}$, Figure 3.2) indicates a free-energy barrier greater than 5.2 $\mathrm{kcal} / \mathrm{mol}$ for the alkane $\mathrm{C}-\mathrm{H}$ bond activation. Nanosecond step-scan FTIR experiments on the $\eta^{3}-\mathrm{Tp} * \mathrm{Rh}(\mathrm{CO})_{2} /$ cyclohexane system. show that the remnant of the $\eta^{2}-\mathrm{Tp} * \mathrm{Rh}(\mathrm{CO})(\mathrm{S})$ peak persists for $\sim 280 \mathrm{~ns}$ after photoexcitation while the product $\mathrm{CO}$ stretch at $2032 \mathrm{~cm}^{-1}$ rises with a time-constant of $230 \mathrm{~ns}$ (Figure 3.5) [15]. Notice that the previously detached pyrazole ring recoordinates to the $\mathrm{Rh}$ center in the final product $\eta^{3}-\mathrm{Tp}_{\mathrm{p}}{ }^{*} \mathrm{Rh}(\mathrm{CO})(\mathrm{H})(\mathrm{R})$. Since no other transient intermediates appear in the time-resolved IR spectra prior to formation of the final product, it is concluded that the rate-limiting step consists of the $\mathrm{C}-\mathrm{H}$ bond cleavage process. At this point, the available experimental data do not allow differentiation of the bond-cleavage step from the ring-rechelation one. Nonetheless, a comparison of the energy barrier of the current system with literature values of analogous systems provides more insight into this reaction. The 230-ns time constant for the last rate-limiting step permits an estimate of the apparent free-energy barrier $\Delta \mathrm{G}^{+} \approx 8.3 \mathrm{kcal} / \mathrm{mol}$. This value is comparable to the $\sim 7.2 \mathrm{kcal} / \mathrm{mol}$ barrier extrapolated from low-temperature studies of the $\mathrm{Cp}{ }^{*} \mathrm{Rh}(\mathrm{CO})_{2}\left(\mathrm{Cp}^{*}=\mathrm{C}_{5} \mathrm{Me}_{5}, \mathrm{Me}=\mathrm{CH}_{3}\right)$ system [79], the rate-limiting step of which is thought to consist of only the $\mathrm{C}-\mathrm{H}$ bond- 
breaking process $[72,80]$. This leads to the proposition of the following rationale for the bond-cleavage step. The $\eta^{2}-\mathrm{Tp}^{*} \mathrm{Rh}^{(\mathrm{l})}(\mathrm{CO})(\mathrm{RH})$ complex with a $\mathrm{Rh}(\mathrm{I})$ center reacts to cleave the $\mathrm{C}-\mathrm{H}$ bond to form the $\left[\eta^{2}-\mathrm{Tp}^{*} \mathrm{Rh}^{(\mathrm{III})}(\mathrm{CO})(\mathrm{R})(\mathrm{H})\right]^{*}$ complex. The latter complex has a electron-deficient $\mathrm{Rh}^{\text {(III) }}$ center, providing the electronic environment for the electron-donating pyrazole ring to reattach to the Rh center on a time scale much shorter than that of $\mathrm{C}-\mathrm{H}$ bond cleavage. The ring-closure drives the reaction to completion and forms the stable final product $\eta^{3}-\mathrm{Tp}{ }^{*} \mathrm{Rh}(\mathrm{CO})(\mathrm{H})(\mathrm{R})$.

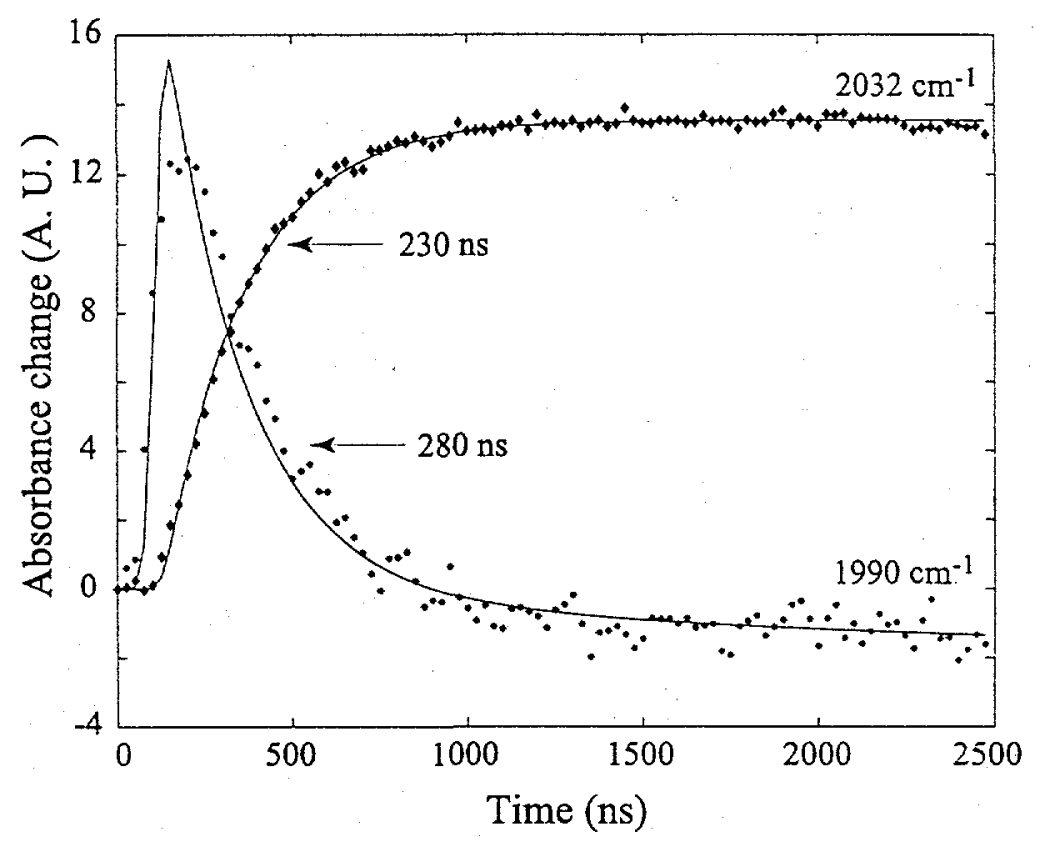

Figure 3.5 Nanosecond kinetics of $\eta^{3}-\mathrm{Tp} * \mathrm{Rh}(\mathrm{CO})_{2}$ in alkane solution.

Nanosecond kinetics (dots) of $\eta^{3}-\mathrm{Tp} * \mathrm{Rh}(\mathrm{CO})_{2}$ in room-temperature alkane solution after $295-\mathrm{nm}$ photolysis at $1990 \mathrm{~cm}^{-1}$, the $\eta^{2}$ intermediate, and $2032 \mathrm{~cm}^{-1}$, the final product.

\subsection{The Reaction Mechanism}

The capability of following the $\mathrm{C}-\mathrm{H}$ bond activation by $\eta^{3}-\mathrm{Tp}{ }^{*} \mathrm{Rh}(\mathrm{CO})$ from its initiation to completion allows one to assemble a comprehensive reaction profile 
delineated in Figure 3.6. The proposed scheme provides a direct assessment of the time scale and energy barrier for the $\mathrm{C}-\mathrm{H}$ bond-breaking step. A comparison of these results to those of the isoelectronic $\mathrm{Si}-\mathrm{H}$ bond activation warrants a better understanding of chemical bonds, to be discussed in the next section.

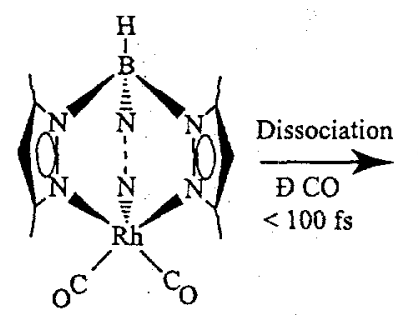

$1981 \mathrm{~cm}^{-1}$ $2054 \mathrm{~cm}^{-1}$
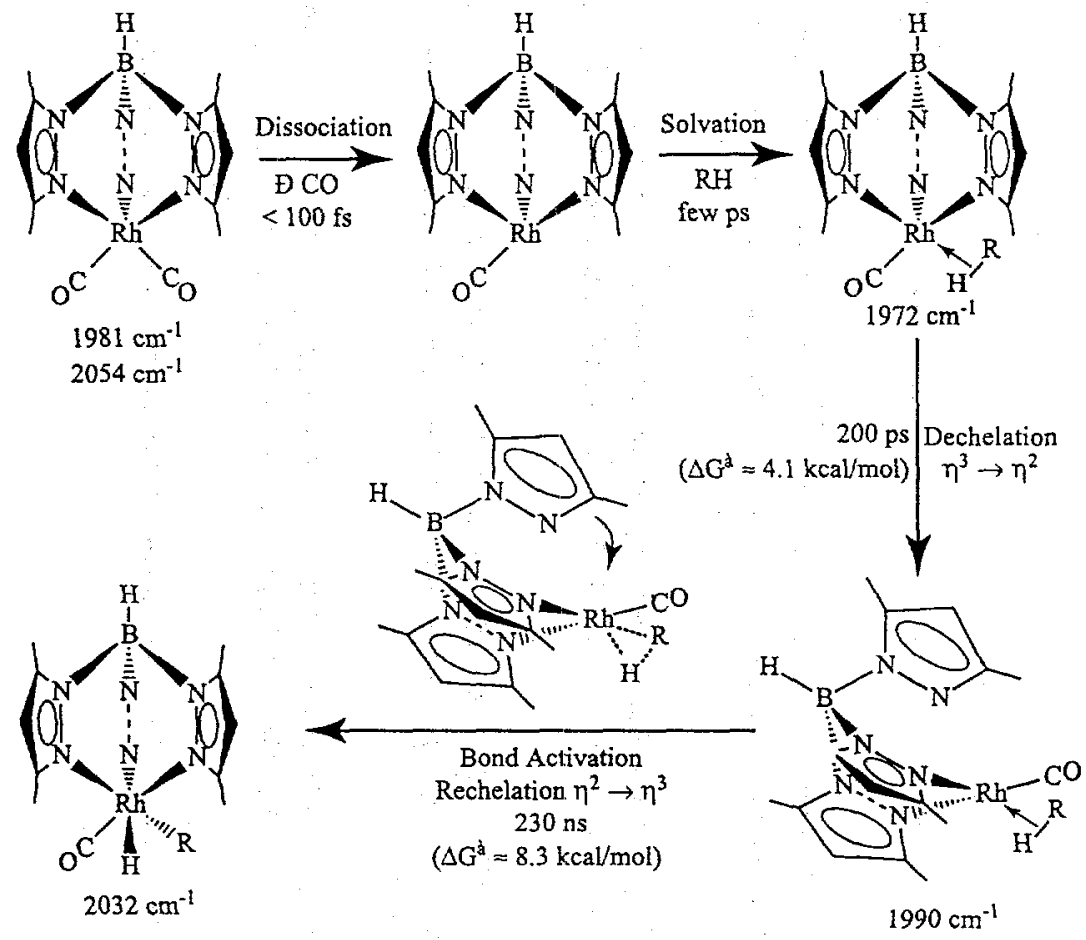

Figure 3.6 A proposed reaction mechanism for the alkane $\mathrm{C}-\mathrm{H}$ bond activation by $\eta^{3}-\mathrm{Tp} \mathrm{p}^{*} \mathrm{Rh}(\mathrm{CO})_{2}$ covering the ultrafast dynamics to nanosecond kinetics.

The dynamics unveiled in this study also address several important issues in related systems. In a study of alkane $\mathrm{C}-\mathrm{H}$ bond activation by the $\mathrm{K}\left[\eta^{2}-\mathrm{Tp}^{*} \mathrm{Pt}^{(\mathrm{II})}\left(\mathrm{CH}_{3}\right)_{2}\right]$ compound, Wick and Goldberg have proposed a dissociative mechanism involving an $\left[\eta^{2}-\mathrm{Tp}^{*} \mathrm{Pt} \mathrm{t}^{(\mathrm{II})}\left(\mathrm{CH}_{3}\right)\right]^{*}$ intermediate prior to formation of the final product $\eta^{3}-\mathrm{Tp}^{*} \mathrm{Pt}^{(\mathrm{IV})}\left(\mathrm{CH}_{3}\right)(\mathrm{R})(\mathrm{H})$ [81]. Similar $\eta^{2}$ intermediates have also been proposed for the vinyl $\mathrm{C}-\mathrm{H}$ bond activation by $\mathrm{Tp}^{*} \mathrm{M}(\mathrm{L})(\mathrm{M}=\mathrm{Rh}, \mathrm{Ir}$; $\mathrm{L}=$ ligand $)$ [82]. For this type of reaction, theoretical studies by Jiménez-Cataño, Niu, and Hall suggest that the two possible $\eta^{2}-\mathrm{Tp}^{*} \mathrm{M}\left(\mathrm{C}_{2} \mathrm{H}_{4}\right)(\mathrm{L})$ and $\eta^{3}-\mathrm{Tp}{ }^{*} \mathrm{M}\left(\mathrm{C}_{2} \mathrm{H}_{4}\right)(\mathrm{L})$ intermediates are connected by a 
small energy barrier. Both intermediates are found to transverse through similar transition states that involve rechelation of a pyrazolyl ligand before reaching the final product $\eta^{3}-\mathrm{Tp}^{*} \mathrm{M}(\mathrm{H})(\mathrm{HCCH} 2)(\mathrm{L})$ [83]. In all the above examples, the $\eta^{3}$-to- $\eta^{2}$ isomerization plays a significant role in the reactivity of the transition-metal complex. In this regard, the reaction scheme in Figure 3.6 not only provides a measure of the relevant time scales but also an understanding of the roles the reactive intermediates assume in the course of a reaction. 


\section{FEMTOSECONd INFRAREd STUdies OF SI-H Bond ACTIVATION BY $\eta^{5}$ - $\operatorname{CPMN}(\mathrm{CO})_{3}$}

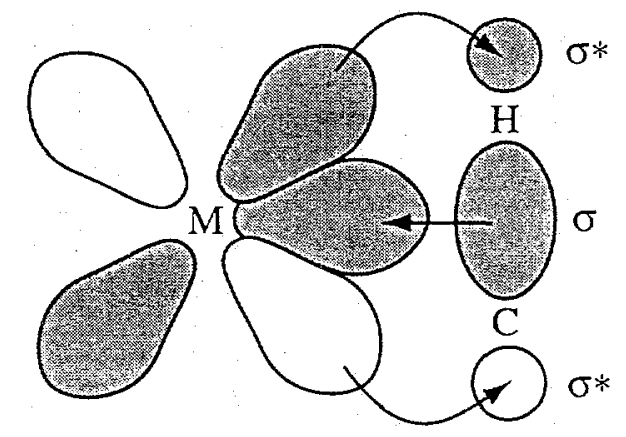

Figure 4.1 A bonding model for $\mathrm{C}-\mathrm{H}$ bond $\sigma$-comple.

The insertion of a transition-metal complex into a $\mathrm{Si}-\mathrm{H}$ bond is an industrially important process in the production of substituted silane and silyl polymers $[2,8 \div]$. The reaction is also helpful for understanding the two-electron oxidative-addition in general through comparison to the isoelectronic $\mathrm{C}-\mathrm{H}$ bond activation [85]. As mentioned earlier, the existence of the proposed $\sigma$-complex intermediate in the $\mathrm{C}-\mathrm{H}$ bond activation has been a critical element in our general understanding of oxidative-addition and reductiveelimination [86]. Analogous to a dihydrogen $\sigma$ complex, in a $\mathrm{C}-\mathrm{H}$ bond $\sigma$-complex the filled $d_{x y}$ orbital of a transition metal overlaps with the $\sigma^{*}$ anti-bonding orbital of an alkane $\mathrm{C}-\mathrm{H}$ bond (cf. Figure 4.1). As a result, the bond order of the otherwise strong $\mathrm{C}-$ $\mathrm{H}$ bond in the $\sigma$ complex is reduced to such an extent that the metal is able to cleave the $\mathrm{C}-\mathrm{H}$ bond under the mild ambient conditions. In a recent theoretical study, Koga, Musaev, and Morokuma have related the activation barriers along a series of $R-H(R=$ $\mathrm{H}, \mathrm{C}, \mathrm{N}, \mathrm{O}$, and $\mathrm{Si}$ ) bonds to factors including the stability of the precursor complex, the $\mathrm{R}-\mathrm{H}$ and $\mathrm{M}-\mathrm{R}$ bond strengths, and the directionality of the $\mathrm{R}-\mathrm{H}$ bond $[8,87]$. They have also predicted that the insertion of a metal complex into a silane $\mathrm{Si}-\mathrm{H}$ bond is a barrierless exothermic reaction, inferring a $\mathrm{Si}-\mathrm{H}$ bond activation timescale on the order 
of solvent coordination time $(\sim$ few ps). The theoretical prediction of the $\mathrm{Si}-\mathrm{H}$ bond activation barrier contrasts those measured at low-temperatures, which suggest an enthalpy-barrier of $7.9 \mathrm{kcal} / \mathrm{mol}$.[88-91] In order to determine the timescale of $\mathrm{Si}-\mathrm{H}$ bond cleavage-thereby the magnitude of the energy barrier-and to understand the nature of the apparent rate-limiting step, a knowledge of the mechanism composed of elementary reaction steps is necessary.

In the initial studies of $\mathrm{Si}-\mathrm{H}$ bond activation, an unexpected reaction intermediate was observed. Two possible assignments were proposed for the assignment of the unknown intermediate: a ring-slipped $\eta^{3}-\mathrm{CpMn}(\mathrm{CO})_{2}$, or an $\eta^{5}-\mathrm{CpMn}(\mathrm{CO})_{2}$ species in a different electronic state. In the Communication that reported these findings, a tentative reaction was proposed assuming a ring-slipped species for the unknown intermediate. To gain further insight into this type of reaction, more experimental and theoretical investigations of the system were conducted. The results from the later studies provided strong evidence supporting a triplet-state assignment for the dicarbonyl species. The rest of this chapter and the next chapter are reproduced from the initial Communication and the full article, respectively, which report our studies of $\mathrm{Si}-\mathrm{H}$ bond activation.

\subsection{Background}

Cleavage, or activation, of the silicon-hydrogen bond of a silane by a metal center has been the focus of many recent studies.[8, 89, 91-94] Knowledge of this type of reaction is relevant to the central ideas of chemical bonding in general and is essential to the development of catalytic reactions such as hydrosilation. It is commonly accepted that the photochemical reaction for the oxidative addition of $\mathrm{Et}_{3} \mathrm{SiH}\left(\mathrm{Et}=\mathrm{C}_{2} \mathrm{H}_{5}\right)$ to $\mathrm{CpMn}(\mathrm{CO})_{3}\left(\mathrm{Cp}=\mathrm{C}_{5} \mathrm{H}_{5}\right)$ begins with the loss of a $\mathrm{CO}$ ligand as the result of $\mathrm{UV}$ irradiation, producing a coordinatively unsaturated dicarbonyl species $[85,88]$ which further reacts to activate the $\mathrm{Si}-\mathrm{H}$ bond to form $\mathrm{CpMn}(\mathrm{CO})_{2}(\mathrm{H})\left(\mathrm{SiEt}_{3}\right)$. Due to the fast 
reaction rates, however, the detailed reaction mechanism including the initial solvation of the nascent photogenerated chemical species has remained unclear. With the advent of ultrafast spectroscopy, especially in the infrared, it is now possible to identify such events that occur on a time scale shorter than diffusion to reveal the underlying elementary reaction steps [16]. In this communication, we report the first ultrafast infrared study on silicon-hydrogen bond cleavage by $\mathrm{CpMn}(\mathrm{CO})_{3}$.

\subsection{Results and Discussions}

As a result of photo-dissociating one $\mathrm{CO}$ ligand, the kinetics of the parent molecule recorded at $2028 \mathrm{~cm}^{-1}$ (A, Figure 4.2) shows an instrument limited bleach that recovers in $32 \pm 7.4$ ps (Figure 4.3 ). ${ }^{1}$ This rapid recovery of the parent bleach is due to vibrational relaxation in the ground electronic manifold $[16,95,96]$. The $31 \%$ recovery is in good agreement with reported $\mathrm{CO}$ loss quantum yields of 0.65 measured at $313-\mathrm{nm}$ excitation [97]. Subsequent spectral evolution suggests that photolysis of the parent molecules leads to two transient species including $\eta^{5}-\mathrm{CpMn}(\mathrm{CO})_{2}$ and a previously unreported intermediate. These reactive intermediates are solvated within few picoseconds [98]. The initial solvation of the nascent species through either the ethyl moiety or the $\mathrm{Si}-\mathrm{H}$ bond of the solvent partitions the reaction into two chamels of disparate time scales. One channel involves a dissociative rearrangement to form the final adduct (pathway II, Figure 4.4), while the other channel includes a direct addition of the $\mathrm{Si}-\mathrm{H}$ bond to the new intermediate, possibly a ring-slipped $\eta^{3}$ dicarbonyl (pathway I, Figure 4.4).

\footnotetext{
${ }^{1}$ Uncertainties represent one standard deviation.
} 


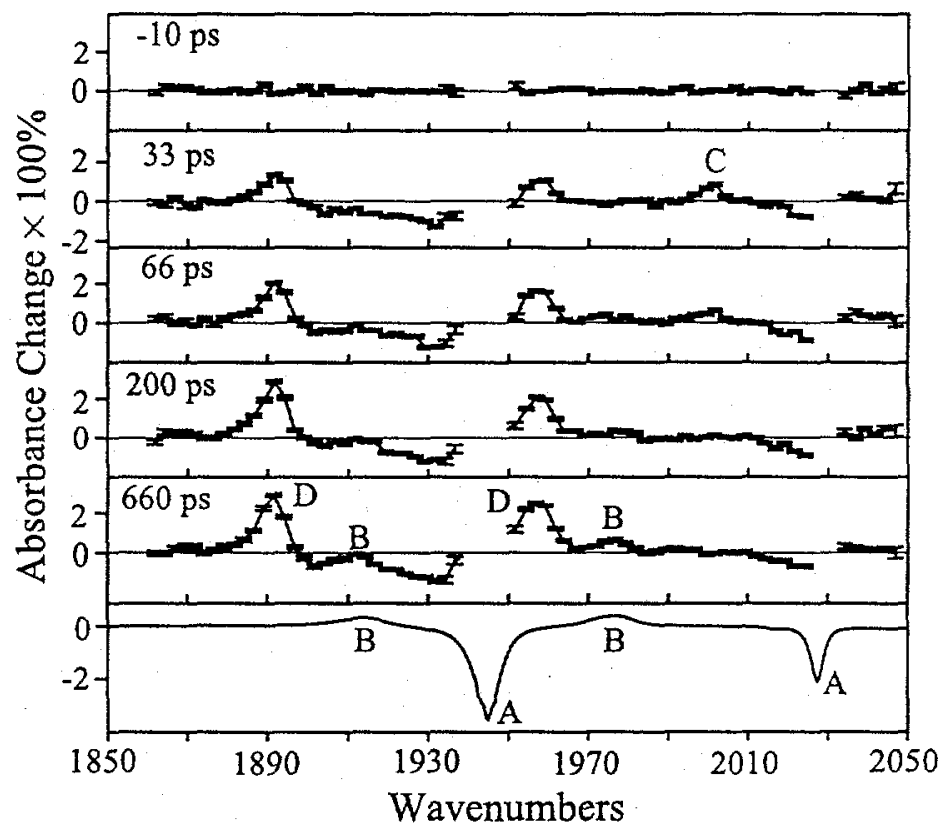

Figure 4.2 Ultrafast IR spectra of $\mathrm{CpMn}(\mathrm{CO})_{3}$ in triethylsilane following 295-nm photolysis.

Transient difference spectra in the $\mathrm{CO}$ stretching region for $\mathrm{CpMn}(\mathrm{CO})_{3}$ in neat triethylsilane at $-10,10,33,66,200$, and 660 ps following 295 -nm UV photolysis. The weak, broad bleaches in the regions $1900-1937$ and $2010-2015 \mathrm{~cm}^{-1}$ can be attributed to the dissociation of naturally abundant ${ }^{13} \mathrm{CO}$ ligands from $\mathrm{CpMn}(\mathrm{CO})_{3}$ [99]. The last panel is an FTIR difference spectrum before and after UV photolysis at $308 \mathrm{~nm}$. Notice that the absorption cross-section of the product (B) is much smaller than that of the parent molecule (A). The large absorption cross-section of the parent $\mathrm{CO}$ bands and the solvent Si-H band $\left(\sim 2100 \mathrm{~cm}^{-1}\right)$ made it very difficult to access some regions of the spectrum under the experimental conditions. A broad, wavelength independent background signal from $\mathrm{CaF}_{2}$ windows has been subtracted from the transient spectra and kinetics. 


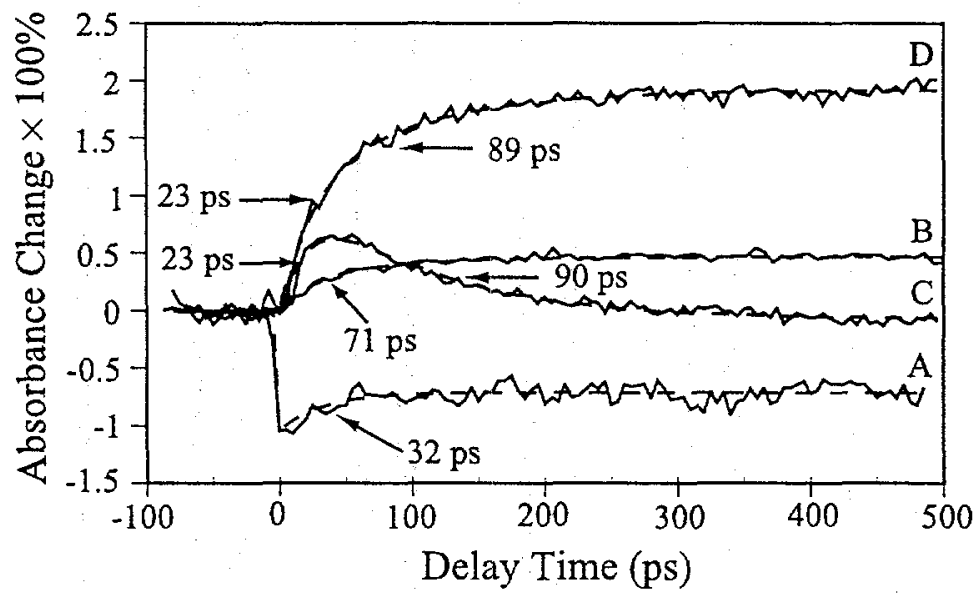

Figure 4.3 Ultrafast IR kinetics of $\mathrm{CpMn}(\mathrm{CO})_{3}$ in triethylsilane following 295-nm photolysis.

Kinetics (solid lines) of $\mathrm{CpMn}(\mathrm{CO})_{3}$ in neat triethylsilane after $295-\mathrm{nm}$ UV photolysis at (A) $2028 \mathrm{~cm}^{-1}$, the parent molecule CO stretch, (B) $1977 \mathrm{~cm}^{-1}$, the CO stretch of the silyl adduct $\mathrm{CpMn}(\mathrm{CO})_{2}(\mathrm{H})\left(\mathrm{SiEt}_{3}\right),(\mathrm{C}) 2002 \mathrm{~cm}^{-1}$, and (D) $1892 \mathrm{~cm}^{-1}$. The wavelengths were chosen to minimize overlap with adjacent peaks. The time constants for the exponential fits (dashed lines) are shown in the graph.

Solvation of $\eta^{5}-\mathrm{CpMn}(\mathrm{CO})_{2}$ and the new intermediate through the ethyl group of $\mathrm{Et}_{3} \mathrm{SiH}$ gives rise to a pair of bands at 1892 and $1957 \mathrm{~cm}^{-1}$ (D, Figure 4.2), and a peak at $2002 \mathrm{~cm}^{-1}$ (C, Figure 4.2), respectively. Assigned to $\eta^{5}-\mathrm{CpMn}(\mathrm{CO})_{2}\left(\mathrm{Et}_{3} \mathrm{SiH}\right), \mathrm{D}$ has also been observed in the low temperature glass studies [100]. Its kinetics displays a biexponential rise of time constants $23 \pm 6.4$ ps and $89 \pm 20$ ps (Figure 4.3D), and maintains the intensity up to $\sim 1 \mathrm{~ns}$. On the other hand, the new transient species $\mathrm{C}$ shows a rise time of $23 \pm 2.3$ ps and a decay time of $90 \pm 6.7$ ps (Figure $4.3 \mathrm{C}$ ). While the 23 ps component of both $\mathbf{C}$ and $\mathbf{D}$ is consistent with vibrational cooling, the similarity between the decay of $\mathbf{C}(90 \mathrm{ps}$ ) and the rise time of $\mathbf{D}$ ( $89 \mathrm{ps}$ ) indicates that $\mathbf{C}$ and $\mathbf{D}$ are kinetically coupled (pathway IIb, Figure 4.4). The identity of $\mathbf{C}$ cannot be a vibrationally excited $\mathbf{A}$ since the latter time constant is greater than the bleach recovery time of $\mathbf{A}$. However, it is very likely that the $\mathbf{C}$ band is due to a dicarbonyl species with the second 
CO stretching band buried in the weak, broad bleach $\left(1900-1937 \mathrm{~cm}^{-1}\right) .{ }^{1}$ Considering its $\sim 45 \mathrm{~cm}^{-1}$ blue shift from the higher energy band of $\mathrm{D}$ and the chemical reactivity of the electron donating $\mathrm{Cp}$ ligand, we tentatively assign $\mathrm{C}$ as an ethyl-solvated $\eta^{3}$ dicarbonyl [75]. This assignment is consistent with the observed $30 \mathrm{~cm}^{-1}$ blue-shifting of the $\mathrm{CO}$ stretching frequency resulting from lowering of the hapticity of $\eta^{3}-\mathrm{Tp}{ }^{*} \mathrm{Rh}(\mathrm{CO})_{2}$ $\left(\mathrm{Tp}^{*}=\right.$ tris-3,5-dimethylpyrazolylborato) to form $\eta^{2}-\mathrm{Tp} * \mathrm{Rh}(\mathrm{CO})[102]{ }^{2}$ With this assignment, the $\sim 90$ ps component can be related to re-chelation from an $\eta^{3}$ to an $\eta^{5}$ species (pathway IIb, Figure 4.4). Presently, we do not know the detailed structure of this intermediate $\mathrm{C}$. Nevertheless, in a separate experiment on $\mathrm{CpMn}(\mathrm{CO})_{3}$ in neat $\mathrm{n}$ pentane, a transient absorption at $2000 \mathrm{~cm}^{-1}$ with similar kinetic behavior was observed, which supports our assigning $\mathbf{C}$ as an ethyl solvate. With these data, however, we cannot exclude possible involvement of other electronic states in explaining the intermediate $\mathbf{C}$. Metastable triplet states have been observed for $\mathrm{Fe}[103,104]$ and $\mathrm{Co}[105,106]$ complexes, and experimental evidence as well as theoretical modeling [107] suggests that transition metal complexes in electronic states of different multiplicity may assume different geometry, which in turn may affect the vibrational frequencies. These considerations will be addressed in the next chapter.

Eventually, on a time scale $>1 \mathrm{~ns}$ as indicated by the absence of $\mathbf{D}$ in the static difference FTIR spectrum (bottom panel, Figure 4.2), D must further rearrange to form the product. For this rearrangement, two mechanisms have been proposed which are the "chain-walk" mechanism [108] and the "dissociative" mechanism [109]. The chain-walk mechanism was first proposed to account for the observed $\sim 100$ ps rearrangement from an alkyl-solvated $\mathrm{Cr}(\mathrm{CO})_{5}(\mathrm{ROH})$ to the more stable hydroxyl-solvated $\mathrm{Cr}(\mathrm{CO})_{5}(\mathrm{HOR})$, where $\mathrm{ROH}$ is 1-propanol. If this is the case in our study, assuming that complexed

\footnotetext{
${ }^{1}$ The gas-phase stretch frequency of the monocarbonyl $\mathrm{CpMn}(\mathrm{CO})$ has been reported to be $1948 \mathrm{~cm}^{-1}$.[101]

${ }^{2}$ Generally, the $\mathrm{CO}$ stretching frequency of a metal carbonyl complex reflects the charge density on the metal center: the less charge the metal center has to back-donate to $\mathrm{CO} \pi^{*}$ orbitals, the higher the $\mathrm{CO}$ stretching frequency will be. Therefore, the $45 \mathrm{~cm}^{-1}$ blue-shift implies an electron deficient metal center for C.
} 
$\mathrm{Et}_{3} \mathrm{SiH}$ migrates from the terminal carbon of one ethyl group to the silicon atom, one would expect the rearrangement to be complete within $100 \mathrm{ps}$. However, the observed decay time of $\mathbf{D}$ is longer than 100 ps, suggesting that a "dissociative" mechanism is dominant. In such a pathway, $\mathrm{CpMn}(\mathrm{CO})_{2}\left(\mathrm{Et}_{3} \mathrm{SiH}\right)$ thermally dissociates slowly into a loosely bound $\mathrm{CpMn}(\mathrm{CO})_{2} \ldots\left(\mathrm{Et}_{3} \mathrm{SiH}\right)$ complex, allowing the metal center to interact with the $\mathrm{Si}-\mathrm{H}$ bond of possibly another solvent molecule. The minimum activation energy for such a dissociative process is then expected to be dictated by the complexation enthalpy of a $\mathrm{CpMn}(\mathrm{CO})_{2}$ and an alkyl group which is estimated to be $\sim 8 \mathrm{kcal} / \mathrm{mol}$ [110]. Ar room temperature, this barrier height corresponds to a reaction time scale of $\sim 120 \mathrm{~ns}$, assuming simple transition-state theory. The observed rearrangement time ( $>1 \mathrm{~ns})$ in this study therefore supports the "dissociative" mechanism (pathway II, Figure 4.4).

Potentially, the bare $\eta^{3}$ species may also be solvated through the $\mathrm{Si}-\mathrm{H}$ bond of the solvent and lead to the product $\eta^{5}-\mathrm{CpMn}(\mathrm{CO})_{2}(\mathrm{H})\left(\mathrm{SiEt}_{3}\right)$ on a time scale similar to the decay of $\mathrm{C}$, when the complex changes its hapticity from $\eta^{3}$ to $\eta^{5}$ thus providing sufficient electron density for the activation process. Indeed, the product band (B, Figure 4.2) exhibits a rise time of $71 \pm 5.4 \mathrm{ps}$ (Figure $4.3 \mathrm{~B}$ ), ${ }^{1}$ indicating that the $\mathrm{Si}-\mathrm{H}$ bond may be activated by direct solvation through the $\mathrm{Si}-\mathrm{H}$ bond of the solvent (pathway I, Figure 4.4). Furthermore, if the activation barrier of a $\mathrm{Si}-\mathrm{H}$ bond is comparably small, the apparent rate determining step would be the dissociative rearrangement from $\mathbf{D}$ to $\mathbf{B}$. Our measured rates for the formation of $\mathbf{B}$ are also consistent with the observation that the overall enthalpy of activation is dominated by the enthalpy of dissociating the solvent from the reaction site, and that the transition state consists of a very loosely bound $\mathrm{R}_{3} \mathrm{SiH}$ $[90,100]$.

\footnotetext{
${ }^{1}$ For the product band, one may expect a biexponential rise similar to $D$ if the $\eta^{5}$ dicarbonyl is also solvated through the Si-H bond of the solvent. However, we did not observe such a behavior. The reason is perhaps due to statistical favor for the ethyl groups over the $\mathrm{Si}-\mathrm{H} \sigma$-bond in a triethylsilane.

Furthermore, one would expect more steric hindrance for the $\eta^{5}$ dicarbonyl to be solvated via the solvent's $\mathrm{Si}-\mathrm{H}$ bond.
} 


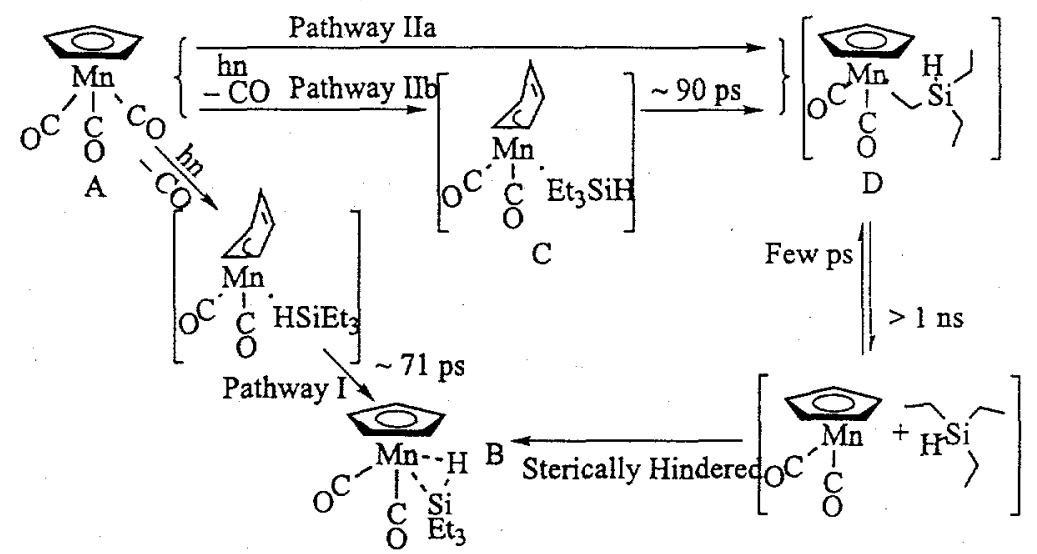

Figure 4.4 A proposed reaction mechanism for the photochemical $\mathrm{Si}-\mathrm{H}$ bond activation of $\mathrm{Et}_{3} \mathrm{SiH}$ by $\mathrm{CpMn}(\mathrm{CO})_{3}$. Suggestive chemical structures are in brackets.

In the light of the above discussion, the overall reaction mechanism is summarized in Figure 4.4. Upon UV excitation, A may lose one CO ligand to form $\eta^{5}-\mathrm{CpMn}(\mathrm{CO})_{2}$, or lose one $\mathrm{CO}$ ligand and at the same time undergo a change in its hapticity to an $\eta^{3}$ species; both dicarbonyls are solvated within few picoseconds. Solvation of the nascent $\eta^{3}$ species via the $\mathrm{Si}-\mathrm{H}$ bond of the solvent leads to the activated product on a time scale of $71 \mathrm{ps}$ (pathway I). Solvation of the $\eta^{3}$ and $\eta^{5}$ dicarbonyls through the ethyl groups of the solvent results in $\mathbf{C}$ and $\mathbf{D}$, respectively, where $\mathbf{C}$ evolves to form $\mathbf{D}$ on a time scale of 90 ps (pathway II). Finally, $\mathbf{D}$ rearranges dissociatively to form the product on a time scale greater than one nanosecond.

\subsection{Conclusion}

This work represents the first study of $\mathrm{Si}-\mathrm{H}$ bond activation at room temperature using ultrafast infrared spectroscopic methods, which are proving to be general and powerful means for studying complex chemical reaction dynamics in condensed phases. Our results suggest that the formation of $\eta^{5}-\mathrm{CpMn}(\mathrm{CO})_{2}(\mathrm{H})\left(\mathrm{SiEt}_{3}\right)$ is partitioned by the initial solvation into two possible pathways: (1) formation through a new intermediate, possibly a photogenerated ring-slipped species, which decays to form the product on a 
time scale of about 71 ps; and (2) formation through an ethyl-solvated dicarbonyl species $\mathrm{CpMn}(\mathrm{CO})_{2}\left(\mathrm{Et}_{3} \mathrm{SiH}\right)$, which eventually dissociatively rearranges to form the product on a time scale greater than $1 \mathrm{~ns}$. This observation is consistent with the low reaction barrier for $\mathrm{Si}-\mathrm{H}$ bond activation, in sharp contrast to $\sim 10 \mathrm{kcal} / \mathrm{mol}$ barrier in activating an isoelectronic $\mathrm{C}-\mathrm{H}$ bond [15]. 


\section{ThE REACTION MECHANISM OF SI-H BOND ACTIVATION STUDIED USING FEMTOSECOND TO NANOSECOND IR SPECTROSCOPY AND AB NITIO METHODS}

\subsection{Introduction}

The photochemical oxidative addition of a $\mathrm{Si}-\mathrm{H}$ bond to certain transition-metal complexes has been the focus of many research efforts since its initial discovery [111]. This type of reaction which cleaves, or activates a $\mathrm{Si}-\mathrm{H}$ bond is critical in hydrosilation [85, 112]. In addition, it provides a direct comparison to another important bondcleavage reaction, $\mathrm{C}-\mathrm{H}$ bond activation by similar transition metal complexes $[15,65$, 67]. As such, knowledge of the reaction mechanism may facilitate the development of homogeneous catalysis $[2,84]$ and lead ultimately to a general understanding of the chemistry of bond-cleavage reactions.

Many of the previous studies have used group VIIB, $d^{6}$ compounds $\eta^{5}-\mathrm{CpM}(\mathrm{CO})_{3}$ ( $M=\mathrm{Mn}, \mathrm{Re}, \mathrm{Cp}=\mathrm{C}_{5} \mathrm{H}_{5}$ ) as model reagents. They provide an interesting contrast of reactivity in that the extent of the $\mathrm{Si}-\mathrm{H}$ bond cleavage varies with the type of metal; the rhenium complex breaks the $\mathrm{Si}-\mathrm{H}$ bond completely whereas there remains residual $\mathrm{Si}$...H interaction in the manganese case. ${ }^{1}$ Mechanistically, Young and Wrighton have established that at low temperatures the primary photochemical product upon UV irradiation of $\mathrm{CpMn}(\mathrm{CO})_{3}$ is the coordinatively unsaturated, 16-e $\mathrm{CpMn}(\mathrm{CO})_{2}$ (Figure 5.1a) [88]. Further studies have shown that the resulting dicarbonyl is complexed by the solvent to form $\mathrm{CpMn}(\mathrm{CO})_{2}$ (solvent), and that the apparent rate-determining step

\footnotetext{
${ }^{1}$ For example, the distance between the silicon and the hydrogen atom in the adduct $\mathrm{CpRe}(\mathrm{CO})_{2}(\mathrm{H})\left(\mathrm{SiPh}_{3}\right)$ is $2.19 \AA\left(\mathrm{Ph}=\right.$ phenyl, $\left.\mathrm{C}_{6} \mathrm{H}_{5}\right)[113]$ compared to $1.80 \AA$ for the similar $\mathrm{Mn}$ compound, $\mathrm{MeCpMn}(\mathrm{CO})_{2}(\mathrm{H})\left(\mathrm{SiPh}_{2} \mathrm{~F}\right)\left(\mathrm{MeCp}=\mathrm{CH}_{3} \mathrm{C}_{5} \mathrm{H}_{4}\right) \cdot[114,115]$ 
involves a loosely solvated transition-state complex $[88-93,100,110,116]$. The reaction of the rhenium complex $\mathrm{CpRe}(\mathrm{CO})_{3}$ with silanes (Figure 5.1b) has also been found to begin with loss of a $\mathrm{CO}$ ligand followed by solvent coordination. Despite the many experimental efforts in deriving the reaction mechanism, elementary reaction steps including the early-time dynamics and the time scale of the $\mathrm{Si}-\mathrm{H}$ bond-breaking step has remained unattainable due to its extremely rapid reaction rate [91]. Recently, our group have been able to investigate the room-temperature reaction dynamics of $\mathrm{Si}-\mathrm{H}$ bond activation by $\mathrm{CpMn}(\mathrm{CO})_{3}$ in neat $\mathrm{Et}_{3} \mathrm{SiH}\left(\mathrm{Et}=\mathrm{C}_{2} \mathrm{H}_{5}\right)$ using femtosecond infrared spectroscopy (Ref. [17], hereafter denoted as Paper I). It was found that UV excitation of the parent compound $\mathrm{CpMn}(\mathrm{CO})_{3}$ generated two dicarbonyls, and subsequent solvation partitioned the reaction into two parallel pathways.

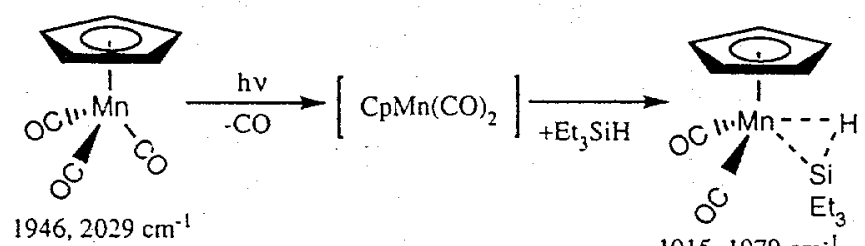

A

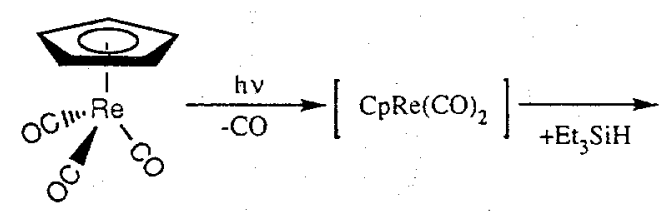

1939. $2030 \mathrm{~cm}^{-1}$

E

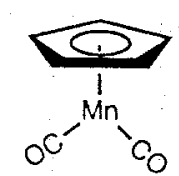

$1883.2000 \mathrm{~cm}^{-1}$

C

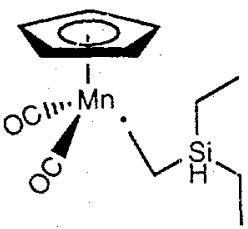

$1892,1960 \mathrm{~cm}^{-1}$

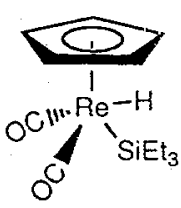

$1918,1990 \mathrm{~cm}^{-1}$

$\mathbf{F}$

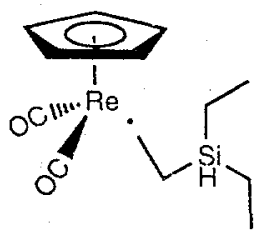

$1887,1948 \mathrm{~cm}^{-1}$

G

Figure 5.1 IR band assignments of chemical species involved in S-H bond activation by $\mathrm{CpM}(\mathrm{CO})_{2}, \mathrm{M}=\mathrm{Mn}, \mathrm{Re}$. 
In this article, we report the use of a combination of theoretical modeling and experimental measurements to continue pursuing this important reaction (Figure 5.1). The elementary reaction steps from initial photolysis to completion of the reaction were studied to derive a comprehensive reaction mechanism. Due to the complexity of the reaction and for clarity, we summarize below some important findings of this work (cf. Figure 5.13 and Figure 5.14) and leave the technicalities for later sections. The final results can be summarized as follows: (1) The early-time reaction dynamics depend critically on the electronic structure of the organometallic complex. For the manganese complex, there are two overlapping electronic bands in accessible UV region (cf. Figure 5.11). The higher-energy band is related to formation of an unsolvated singlet dicarbonyl $\mathbf{D}^{*}$ (an asterisk indicates excited state) and the lower one to triplet dicarbonyl C. A typical UV excitation of the parent molecule will populate both states, resulting in $\mathbf{C}$ or $\mathbf{D}^{*}$ through parallel reaction channels. The branching ratios $\mathbf{A} \rightarrow \mathbf{C} \rightarrow \mathbf{D}: \mathbf{A} \rightarrow \mathbf{D}^{*} \rightarrow \mathbf{D}$ for these two parallel channels measured at $295-\mathrm{nm}$ and $325-\mathrm{nm}$ excitations are approximately 50\%:50\% and 75\%:25\%, respectively. For the rhenium complex. there appears only one electronic band in the UV region; photolysis of the rhenium parent molecule leads exclusively to a singlet rhenium dicarbonyl $G^{*}$. (2) For both metal complexes, initial solvation of the nascent coordinatively unsaturated dicarbonyls $\left(C, D^{*}\right.$, and $\mathbf{G}^{*}$ ) either via the ethyl moiety or the $\mathrm{Si}-\mathrm{H}$ bond of the solvent molecule $\mathrm{Et}_{3} \mathrm{SiH}$ partitions the reaction into two pathways. Solvation of the metal dicarbonyl through the $\mathrm{Si}-\mathrm{H}$ bond leads directly to the final products $\mathbf{B}$ or $\mathbf{F}$ on the ultrafast time scale. Solvation through an ethyl group results in reactive intermediates $\mathbf{D}$ or $\mathbf{G}$, which reacts further to form the final products in $177 \mathrm{~ns}$ and $6.8 \mu \mathrm{s}$, respectively. (3) The relative probabilities of (solvation through the ethyl moiety):(solvation through the $\mathrm{Si}-\mathrm{H}$ bond) for the $\mathrm{Mn}$ and Re complexes are 5.3 and 3, respectively. The differences are attributed to steric effects. The larger diameter of the rhenium atom allows equivalent sampling of the chemically different sites in a solvent molecule ( 3 ethyl groups and $1 \mathrm{Si}-\mathrm{H}$ bond). (4) The apparent rate-determining step for both reactions is the dissociative rearrangement 
from the ethyl solvates $\mathbf{D}$ or $\mathbf{G}$ to the final products. The corresponding free-energy barriers for the manganese and rhenium complexes, determined from simple transitionstate theory, are $8.25 \pm 0.03 \mathrm{kcal} / \mathrm{mol}$ and $10.41 \pm 0.02 \mathrm{kcal} / \mathrm{mol}$, respectively. (5) The time scale for a $\mathrm{Si}-\mathrm{H}$ bond-breaking process is for the first time estimated to be $4.4 \pm 2.6$ ps, indicative of a low barrier, if any, reaction.

The paper is organized as follows: The results and detailed data analysis are presented in 5.2, in the order of manganese/experimental, rhenium/experimental. and theoretical results. Implications of the results are discussed in 5.3. In particular, for the manganese complex, we discuss the effects of sampling different regions of electronically excited potential energy surface, the nature of the observed transient intermediates, and their interaction with solvent. Comprehensive reaction mechanisms are described in the end of Section 5.3.1 for Mn and in 5.3.2 for Re. Finally, current results are compared to the $\mathrm{C}-\mathrm{H}$ bond activation in 5.4 .

\subsection{Results}

The infrared spectra in the $\mathrm{CO}$ stretching region are presented in a form of difference absorbance in which positive bands indicate the appearance of new species while negative bands (bleaches) represent the depletion of parent molecules. In the ultrafast spectra of each metal complex, the bottom panel is an FTIR difference spectrum of the sample taken a few minutes after prolonged exposure to $308-\mathrm{nm}$ UV pulses from an excimer laser.

\subsubsection{Activation of the Silicon-Hydrogen Bond of $\mathrm{Et}_{3} \mathrm{SiH}$ by $\mathrm{CpMn}(\mathrm{CO})_{3}$}




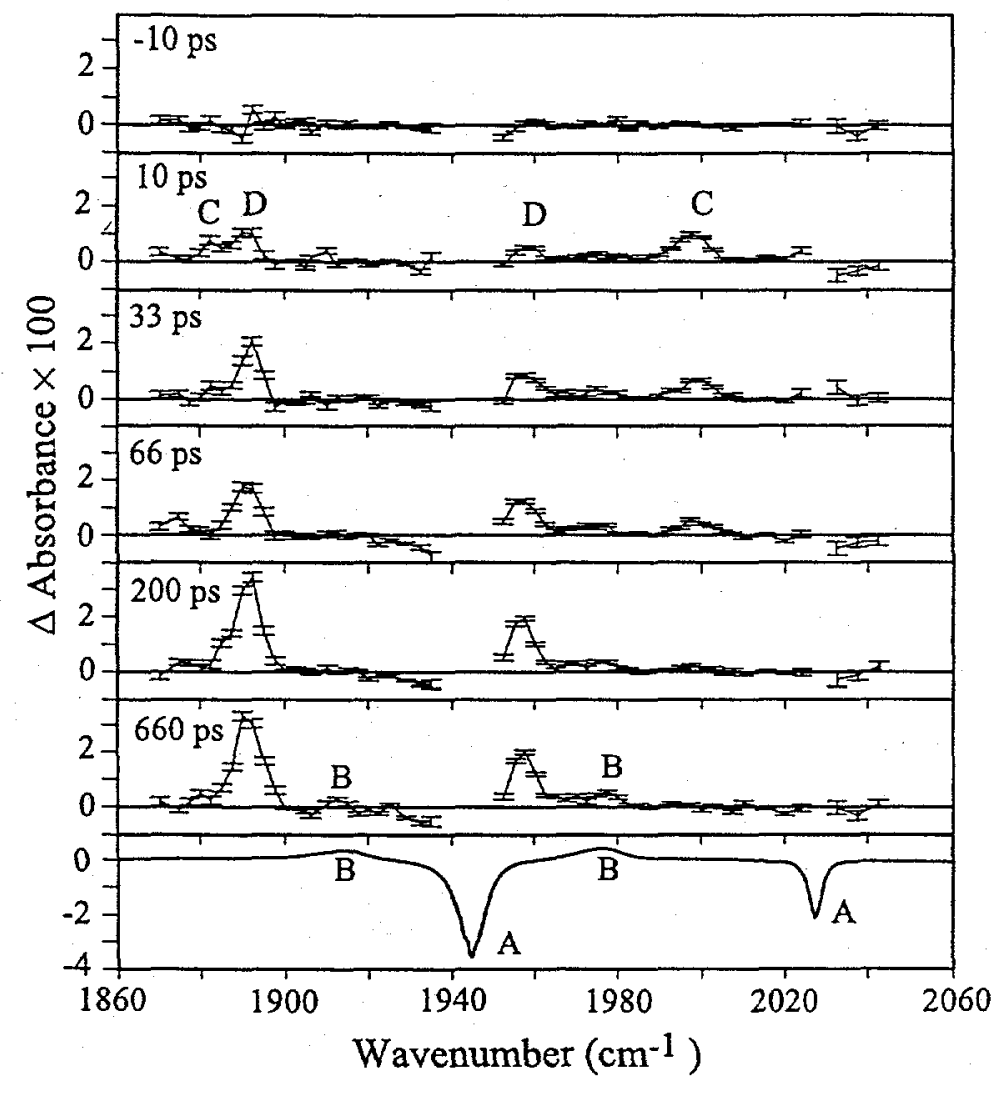

Figure 5.2 Ultrafast IR spectra of $\mathrm{CpMn}(\mathrm{CO})_{3}$ in triethylsilane following $325-\mathrm{nm}$ excitation.

Transient difference spectra in the $\mathrm{CO}$ stretching region for $\mathrm{CpMn}(\mathrm{CO})_{3}$ in neat triethylsilane at $-10,10,33,66,200$, and 660 ps following 325 -nm UV photolysis. Under the experimental conditions, the large cross-section of the solvent $\mathrm{Si}-\mathrm{H}$ band $\left(\sim 2100 \mathrm{~cm}^{-1}\right)$ and the parent $\mathrm{CO}$ bands $\left(1947\right.$ and $\left.2028 \mathrm{~cm}^{-1}\right)$ make it difficult to access some regions of the spectrum. ${ }^{1}$. The last panel is an FTIR difference spectrum before and after UV photolysis at $308 \mathrm{~nm}$.

\footnotetext{
${ }^{1}$ The extinction coefficients $\varepsilon$ for $v(\mathrm{CO})$ of $\mathrm{CpMn}(\mathrm{CO})_{3}$ are $12700 \mathrm{M}^{-1} \mathrm{~cm}^{-1}$ for the 1947 - and 2028- $\mathrm{cm}^{-1}$ peaks, respectively.[100] To prepare a sample which has an OD $\sim 1$ at the pump wavelength of $325 \mathrm{~nm}$, the IR absorbance of the 1947- and $2028-\mathrm{cm}^{-1}$ bands are calculated to be 9.2 and $3.3 \mathrm{OD}$, respectively ( 9 $\mathrm{mM}, 630-\mu \mathrm{m}$ cell). A thinner cell was used to acquire kinetics for the parent molecule. Such an experimental difficulty is expected when the sample has a low absorption cross section in the UV but a high extinction coefficient in the probe IR region. A sample calculation to estimate the signal strength is included in footnote 1 on page 63.
} 


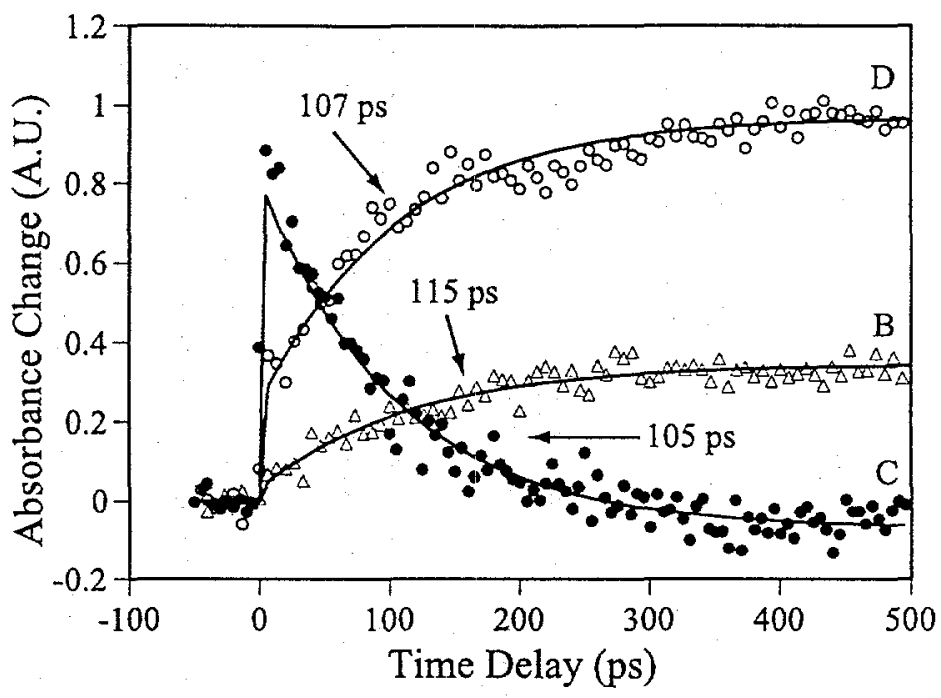

Figure 5.3 Ultrafast IR kinetics of $\mathrm{CpMn}(\mathrm{CO})_{3}$ in triethylsilane following $325-\mathrm{nm}$ excitation.

Ultrafast kinetics of $\mathrm{CpMn}(\mathrm{CO})_{3}$ in neat triethylsilane after $325-\mathrm{nm}$ UV photolysis at (B) $1979 \mathrm{~cm}^{-1}$, the $\mathrm{CO}$ stretch of the silyl adduct $\mathrm{CpMn}(\mathrm{CO})_{2}(\mathrm{H})\left(\mathrm{SiEt}_{3}\right)$ (open triangles); (C) $2002 \mathrm{~cm}^{-1}$ (filled circles); (D) $1892 \mathrm{~cm}^{-1}$ (open circles). The wavelengths were chosen to minimize overlap with adjacent peaks. The time constants for the exponential fits (dashed lines) are shown in the graph.

Shown in Figure 5.2 are the fs-IR spectra of $\eta^{5}-\mathrm{CpMn}(\mathrm{CO})_{3}$ in neat $\mathrm{Et}_{3} \mathrm{SiH}$ following excitation at $325 \mathrm{~nm}$. The peaks at 1892 and $1960 \mathrm{~cm}^{-1}$ are assigned to $\eta^{5}-\mathrm{CpMn}(\mathrm{CO})_{2}\left(\mathrm{Et}_{3} \mathrm{SiH}\right)$, denoted $\mathbf{D}$ (Figure 5.1). Having also been observed in lowtemperature studies [91, 100], $\mathrm{D}$ is attributed to solvation of $\eta^{5}-\mathrm{CpMn}(\mathrm{CO})_{2}$ through the ethyl moiety of $\mathrm{Et}_{3} \mathrm{SiH}$. In the 10 ps panel of Figure 5.2, the two bands at 2000 and $\sim 1883 \mathrm{~cm}^{-1}$ which disappear at longer time scales are attributed to a triplet dicarbonyl species, denoted $\mathbf{C}$ (see 5.3.1 for assignment). Figure 5.3 shows the kinetic traces of $\mathbf{D}$, $\mathbf{C}$, and the final product $\mathbf{B}$ following 325-nm excitation. The kinetics of $\mathbf{C}$ recorded at $2000 \mathrm{~cm}^{-1}$ exhibit a rapid rise and an $105 \pm 8.0$ ps decay. ${ }^{1}$ The product band at $1979 \mathrm{~cm}^{-1}$ displays a single exponential rise of $115 \pm 14$ ps.

\footnotetext{
${ }^{1}$ Uncertainties represent one standard deviation.
} 
The kinetic trace of $\mathbf{D}$ at $1892 \mathrm{~cm}^{-1}$ exhibits a fast rise, then gradually grows to a constant level. It can be described by the equation,

Equation 5.1 $\quad \operatorname{Signal}(t)=C_{1}\left(1-e^{-t / \tau}\right)+C_{2}$,

where $\tau$ is a formation time constant, and $C_{1}$ and $C_{2}$ are amplitude constants. This same equation will be used later in modeling kinetic traces of similar behavior. The fitted numeric values for $\tau, C_{1}$, and $C_{2}$ are $107 \pm 8.9$ ps, $0.73 \pm 0.033$, and $0.25 \pm 0.033$, respectively. Clearly, the decay of the intermediate $\mathbf{C}$ is correlated to the formation of both the ethyl-solvate $\mathbf{D}$ and the final product $\mathbf{B}$. Equation 5.1 implies two channels for the formation of the ethyl solvate $\eta^{5}-\mathrm{CpMn}(\mathrm{CO})_{2}\left(\mathrm{Et}_{3} \mathrm{SiH}\right)$, the branching ratio of which is represented by the relative magnitude of $C_{1}$ to $C_{2}$. With a typical solvation time of a few ps in mind, the channel that contributes to the fast rise $C_{2}$ of the transient of $D$ is attributed to solvation of a singlet $\eta^{5}-\mathrm{CpMn}(\mathrm{CO})_{2}$, denoted $\mathrm{D}^{*}$. The other channel that contributes to the 107 ps rise is attributed to interconversion from $\mathbf{C}$. Consequently, the branching ratio of the reaction pathway $\mathbf{A} \rightarrow \mathbf{C} \rightarrow \mathbf{D}$ to the pathway $\mathbf{A} \rightarrow \mathbf{D}^{*} \rightarrow \mathbf{D}$ in this case is approximately $3: 1$. The implications of these results will be discussed in 5.3.

The long-time behavior of the reaction was studied using a nanosecond step-scan FTIR spectrometer. Displayed in Figure 5.4 are the kinetics of $\mathbf{D}$ and that of the product B, recorded at $1892 \mathrm{~cm}^{-1}$ and $1979 \mathrm{~cm}^{-1}$, respectively. D shows an instrument-limited rise and then decays with a time constant of $183 \pm 8 \mathrm{~ns}$. B exhibits a fast rise and an exponential growth of $177 \pm 7 \mathrm{~ns}$. The rapid rise of $\mathbf{B}$ is from the remnant of the initial $\mathrm{Si}-\mathrm{H}$ solvate that forms in the ultrafast time scale. As has been discussed in Paper $\mathrm{I}$, the latter exponential rise of $\mathbf{B}$ is due to the dissociative rearrangement from $\mathbf{D}[17,109]$. The nanosecond kinetics of $\mathbf{B}$ can also be modeled with Equation 5.1. In this case the coefficients $C_{1}$ and $C_{2}$ represent the relative probability of the initial solvation through the ethyl moiety $(84 \%)$ or the Si-H bond $(16 \%)$, respectively. These numbers, however, should be treated in a qualitative manner. The greater excitation photon flux in our 
nanosecond setup compared to the femtosecond one may cause a transient temperature increase in the sample, which in turn may result in a time-dependent change in the index of refraction [117]. Such a thermal effect makes it difficult to quantify the observed kinetics rigorously.

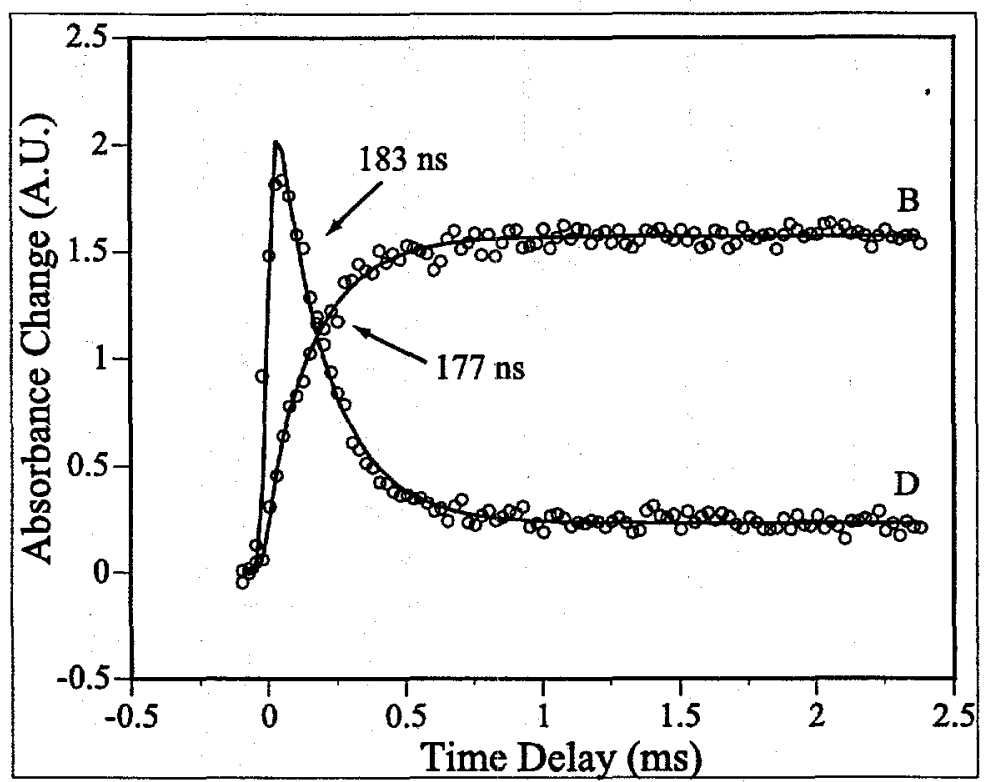

Figure 5.4 Nanosecond kinetics of $\mathrm{CpMn}(\mathrm{CO})_{3}$ in neat triethylsilane following 295-nm excitation.

Nanosecond kinetics (open circles) of $\mathrm{CpMn}(\mathrm{CO})_{3}$ in neat triethylsilane after 295-nm UV photolysis at (B) $1979 \mathrm{~cm}^{-1}$, the CO stretch of the silyl adduct $\mathrm{CpMn}(\mathrm{CO})_{2}(\mathrm{H})\left(\mathrm{SiEt}_{3}\right)$; (D) $1892 \mathrm{~cm}^{-1}$. The finite intensity of the $1892 \mathrm{~cm}^{-1}$ kinetic trace at longer delay time is probably due to $\mathrm{CpMn}\left({ }^{13} \mathrm{CO}\right)\left({ }^{12} \mathrm{CO}\right)(\mathrm{H})\left(\mathrm{SiEt}_{3}\right)$ which has a $\mathrm{CO}$ absorption at $\sim 1887 \mathrm{~cm}^{-1}$ (footnote 1 of page 65). The time constants for the exponential fits (dashed lines) are shown in the graph.

Finally, the free-energy barrier of the dissociative process from $\mathbf{D}$ to $\mathbf{B}$ can be derived from the kinetic traces. If one assumes a simple transition-state theory, the reaction rate can be expressed as the reciprocal of the observed lifetime $\tau$, 


\section{Equation 5.2 \\ Reaction rate $\approx \frac{1}{\tau}=\frac{k_{B} T}{h} e^{-\frac{\Delta G^{+}}{R T}}$,}

where $\mathrm{k}_{\mathrm{B}}$ is the Boltzmann constant, $h$ Plank's constant, $T$ room temperature $(298.15 \mathrm{~K}$ ), and $\Delta \mathrm{G}^{+}$the free energy of activation. The reaction barrier, estimated from the product formation time, is $\Delta G^{+}=8.25 \pm 0.03 \mathrm{kcal} / \mathrm{mol}$. This is in excellent agreement with the literature value, $8.56 \pm 0.49 \mathrm{kcal} / \mathrm{mol}$, which is extrapolated to room temperature using the activation parameters obtained from low temperature measurements [100].

\subsubsection{Activation of the Silicon-Hydrogen Bond of Et $t_{3} \mathrm{SiH}$ by $\mathrm{CpRe}(\mathrm{CO})_{3}$}

Displayed in Figure 5.5 are the transient fs-IR spectra of $\mathrm{CpRe}(\mathrm{CO})_{\text {s }}$ in neat $\mathrm{Et}_{3} \mathrm{SiH}$ following excitation at $295 \mathrm{~nm}$. The bottom panel is an FTIR difference spectrum taken after prolonged exposure to $308-\mathrm{nm}$ light. The presence of naturally abundant ${ }^{13} \mathrm{CO}$ stretches is evidenced by the enlarged FTIR spectrum. ${ }^{1}$

\footnotetext{
${ }^{1}$ In the following, we'll use the Re system as an example to estimate the signal size of $\mathbf{F}$ in the ultrafast spectra. The same calculation is applicable to Mn spectra. We start by calculating the OD change in one of the parent bands, say the $2030 \mathrm{~cm}^{-1}$ band. The pump beam consists of $295-\mathrm{nm}$ pulses of energy $-6 \mu \mathrm{J}, 75 \%$ of which are absorbed by an $\mathrm{OD} \sim 0.6$ sample. Change in the parent absorbance is calculated by $\triangle \mathrm{OD}(\mathrm{E})=$ $\varepsilon \times l \times \Delta c_{\text {parent, }}$, where $l$ is the sample cell thickness, and $\Delta c_{\text {parent }}$ is the concentration change of parent species in the cylindric volume defined by the pump beam. Taking into account the cell thickness $(650 \mu \mathrm{m})$, the beam size at the sample $(-200 \mu \mathrm{m})$, quantum yield $(\sim 0.3)$, each pump pulse induces OD change in the parent bleach $\sim 0.06$. The signal size of $\mathbf{F}$ on the ultrafast time scale can be expressed as $\Delta \mathrm{OD}(\mathbf{F})=\Delta \mathrm{OD}(\mathbf{E}) \times \mathrm{X}(\mathbf{F}) \times \varepsilon(\mathbf{F}) / \varepsilon(\mathbf{E})$,

where $X(F)=0.248 \pm 0.079$ is the branching percentage for $\mathbf{F}$ measured from the nanosecond kinetics, and the extinction coefficient ratio $\varepsilon(\mathbf{F}) / \varepsilon(\mathbf{E}) \sim 2 / 5$ measured from the static difference FTIR spectrum on the bottom panel of Figure 5.5. $\triangle O D(F)$ is thus calculated to be $\sim 0.006$, which is consistent with what we have measured in the present work. The above calculation also serves as a cross-examination for the measured $25 \%$ branching ratio in the initial solvation through the $\mathrm{Si}-\mathrm{H}$ bond of $\mathrm{Et}_{3} \mathrm{SiH}$.

The recorded ${ }^{13} \mathrm{C}$ bleach signal in the ultrafast spectra of the Re complex may appear somewhat larger than one would have expected from a simple estimate. In the following. we show that the Re spectra displayed reasonable ${ }^{13} \mathrm{C}$ bleach at $2015 \mathrm{~cm}^{-1}$. Take the $200 \mathrm{ps}$ panel of Figure 5.5 as an example. The numerical values for the $1990 \mathrm{~cm}^{-1}$ band of $\mathbf{F}$ and the ${ }^{13} \mathrm{C}$ bleach at $2015 \mathrm{~cm}^{-1}$ are about $0.0053 \pm 0.0005$ $\mathrm{OD}$ and $-0.0036 \pm 0.001 \mathrm{OD}$, respectively. Assuming $3 \%$ natural abundance of ${ }^{13} \mathrm{C}$, and taking into account the difference in extinction coefficients $\varepsilon(\mathbf{F}) / \varepsilon\left({ }^{13} \mathrm{C}\right) \sim 2 / 5$ and branching ratios $0.248 \pm 0.079$, one finds that the estimated signal size of the ${ }^{13} \mathrm{C}$ bleach is $\sim-0.0017 \pm 0.0005 \mathrm{OD}$, within a $95 \%$ confidence interval compared to recorded signal size $(-0.0036 \pm 0.001 \mathrm{OD})$. Similarly, the corresponding
} 


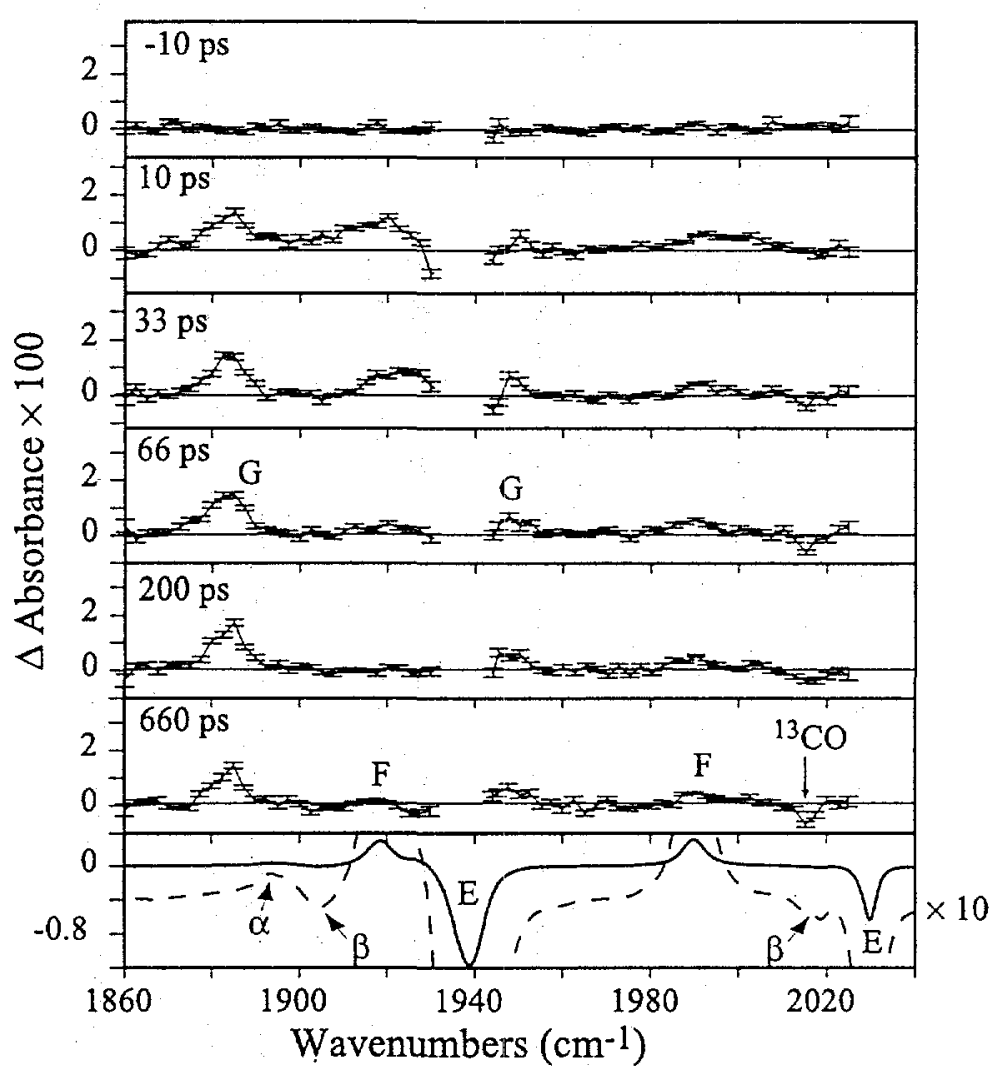

Figure 5.5 Ultrafast IR spectra of $\mathrm{CpRe}(\mathrm{CO})_{3}$ in triethylsilane following $295-\mathrm{nm}$ excitation.

Transient difference spectra in the $\mathrm{CO}$ stretching region for $\mathrm{CpRe}(\mathrm{CO})_{3}$ in neat triethylsilane at $-10,10,33,66,200$, and 660 ps following $295-\mathrm{nm}$ UV photolysis. The arrow at $-2015 \mathrm{~cm}^{-1}$ indicates the bleach due to photolysis of the naturally abundant $\mathrm{CpRe}\left({ }^{12} \mathrm{CO}\right)_{2}\left({ }^{13} \mathrm{CO}\right)$ (footnote 1 on page 63). Last panel: An FTIR difference spectrum before and after UV photolysis at $308 \mathrm{~nm}$. Enlarged spectrum (10X and offset by $-0.4 \%)$ is displayed in dashed line to show $(\alpha)$ a peak of $\operatorname{CpRe}\left({ }^{13} \mathrm{CO}\right)\left({ }^{12} \mathrm{CO}\right)(\mathrm{H})\left(\mathrm{SiEt}_{3}\right)$ at $\sim 1892 \mathrm{~cm}^{-1}$; and $(\beta)$ bleaches of $\mathrm{CpRe}\left({ }^{13} \mathrm{CO}\right)\left({ }^{12} \mathrm{CO}\right)_{2}$ at $\sim 1906$ and $\sim 2019 \mathrm{~cm}^{-1}$.

${ }^{13} \mathrm{C}$ bleach of $\mathrm{Mn}$ complex has a signal size of $-0.0027 \pm 0.0006$ OD (taken from the 660 ps panel of Figure 5.2), which is also reasonable within experimental error. 
The small peak at $1892 \mathrm{~cm}^{-1}$ is assigned to $\eta^{5}-\mathrm{CpRe}\left({ }^{13} \mathrm{CO}\right)\left({ }^{12} \mathrm{CO}\right)(\mathrm{H})\left(\mathrm{SiEt}_{3}\right)$, denoted $\alpha$ in the enlarged spectrum. The shallow bleaches labeled as $\beta$ are due to depletion of naturally abundant $\eta^{5}-\mathrm{CpRe}\left({ }^{12} \mathrm{CO}\right)_{2}\left({ }^{13} \mathrm{CO}\right) .{ }^{1}$ At the 10 ps panel, the pair of bands at $1887 \mathrm{~cm}^{-1}$ and $1948 \mathrm{~cm}^{-1}$ are assigned to $\eta^{5}-\mathrm{CpRe}(\mathrm{CO})_{2}\left(\mathrm{Et}_{3} \mathrm{SiH}\right)(\mathrm{G})$, resulting from solvation of the $\eta^{5}-\mathrm{CpRe}(\mathrm{CO})_{2}\left(\mathbf{G}^{*}\right)$ through the ethyl moiety of the solvent [100]. The final product $\mathbf{F}$ appears as two bands at $1918 \mathrm{~cm}^{-1}$ and $1990 \mathrm{~cm}^{-1}$, but its early-time spectra are complicated by absorptions from vibrationally excited parent molecules $\mathrm{E}$ (the 10 and 33 ps panels).

As shown in Figure 5.6a, the kinetics of the parent molecule E taken at $2030 \mathrm{~cm}^{-1}$ display an instrument-limited depletion which recovers with a time constant of $39.3 \pm 1.6$ ps to $28 \%$ of its initial intensity, consistent with the literature quantum yields of 0.30 at 313-nm excitation [97]. The recovery of the parent bleach is attributed to re-establishing the thermal distribution of vibrational states in the electronic-ground manifold $[16,95$, $96,98,120]$. Processes that contribute to this non-equilibrated vibrational distribution include depletion of the $v=0$ state by the pump photon, and population replenished by non-dissociative processes. As a consequence of populating the higher vibrational states, transitions such as $v=1 \rightarrow 2$ and $v=2 \rightarrow 3$ would become detectable. The broad band at $\sim 1921 \mathrm{~cm}^{-1}$ is assigned to the $\mathrm{v}=1 \rightarrow 2$ absorption of the $1939 \mathrm{~cm}^{-1} \mathrm{CO}$ stretching band of $\mathbf{E}$, which evolves to the blue end of the spectrum and decays completely at later time (10 ps panel, Figure 5.5). The observed $\sim 18 \mathrm{~cm}^{-1}$ anharmonicity is consistent with the reported anharmonicity of other metal carbonyls $[16,95,96,120]$.

${ }^{1} \mathrm{CO}$ frequencies of $\left.\mathrm{CpRe}\left({ }^{13} \mathrm{CO}\right)_{x}{ }^{12} \mathrm{CO}\right)_{3-\mathrm{x}}$ in room-temperature alkane solutions were estimated from an effective force field calculation following the methods in Refs. $[118,119]$. The calculated frequencies (in $\left.\mathrm{cm}^{-1}\right)$ are $\mathrm{CpRe}\left({ }^{12} \mathrm{CO}\right)_{3}\{1939,2030\} ; \mathrm{CpRe}\left({ }^{13} \mathrm{CO}\right)\left({ }^{12} \mathrm{CO}\right)_{2}\{1906,1939,2019\} ; \mathrm{CpRe}\left({ }^{13} \mathrm{CO}\right)_{2}\left({ }^{12} \mathrm{CO}\right)\{1895$, 1919, 2005\}; and $\mathrm{CpRe}\left({ }^{13} \mathrm{CO}\right)_{3} \quad\{1895,1985\}$. Similarly, peaks originating from $\mathrm{CpM}\left({ }^{13} \mathrm{CO}\right)\left({ }^{12} \mathrm{CO}\right)(\mathrm{H})\left(\mathrm{SiEt}_{3}\right)$ are estimated to be at 1892 and $1974 \mathrm{~cm}^{-1}$ for the rhenium complex and at 1887 and $1964 \mathrm{~cm}^{-1}$ for the manganese complex. 


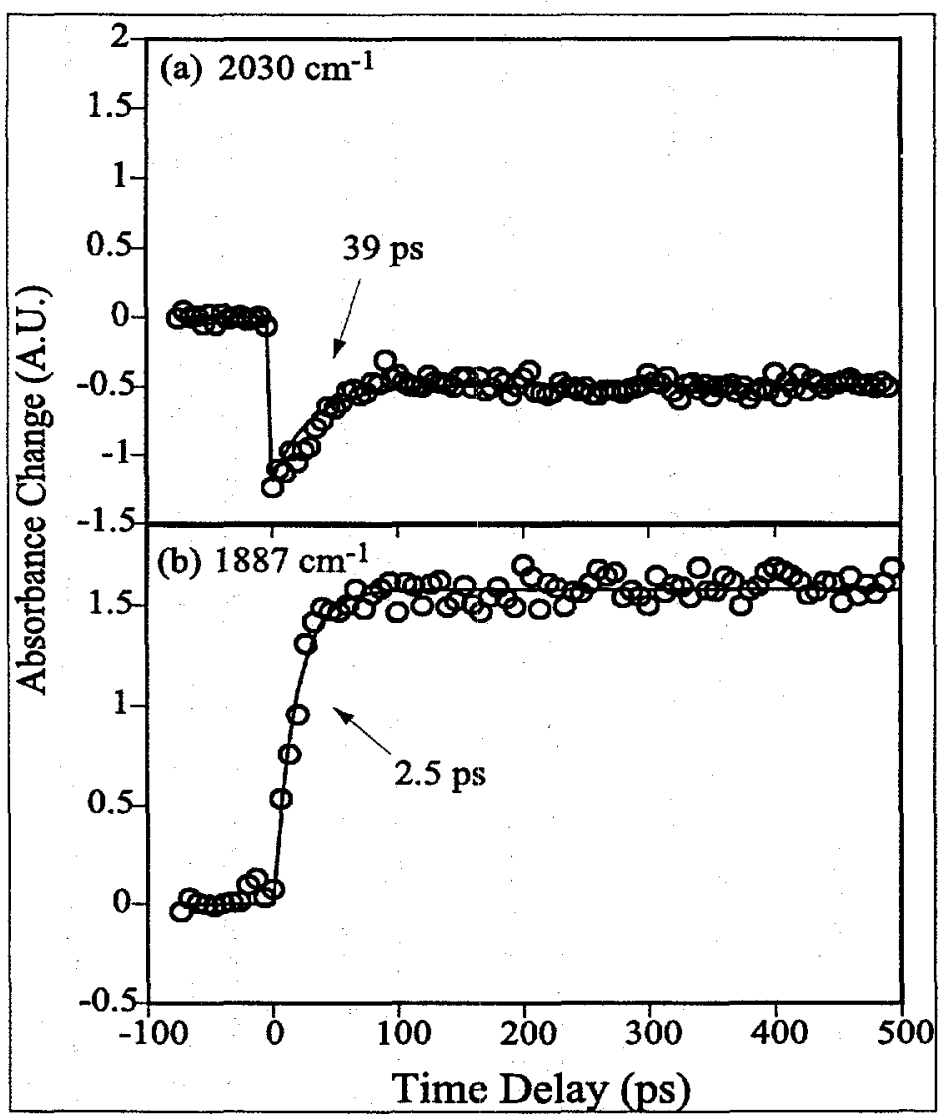

Figure 5.6 Ultrafast IR kinetics of $\mathrm{CpRe}(\mathrm{CO})_{3}$ in triethylsilane following 295-nm excitation.

Ultrafast kinetics (open circles) of $\mathrm{CpRe}(\mathrm{CO})_{3}$ in neat triethylsilane after 295-nm UV photolysis at (a) $2030 \mathrm{~cm}^{-1}$, the A-band of the $\mathrm{CO}$ stretches of the parent molecule. A $100-\mu \mathrm{m}$ cell was used to record this kinetic trace; (b) $1887 \mathrm{~cm}^{-1}$. The time constants obtained from exponential fits (solid lines) to the kinetic traces are also shown in the graph.

Similarly, the broad bands at $\sim 2004 \mathrm{~cm}^{-1}$ and $\sim 1990 \mathrm{~cm}^{-1}$ in the $10 \mathrm{ps}$ panel of Figure 5.5 can be assigned to the $\mathrm{v}=1 \rightarrow 2$ and $\mathrm{v}=2 \rightarrow 3$ absorption of naturally abundant $\mathrm{CpRe}\left({ }^{13} \mathrm{CO}\right)(\mathrm{CO})_{2}$, respectively. The parent molecules containing one ${ }^{13} \mathrm{CO}$ ligand manifest themselves as a depletion at $2015 \mathrm{~cm}^{-1}$ as indicated by the arrow in the 660 -ps panel of Figure 5.5 (see footnote 1 of page 65 for estimation of $\mathrm{CO}$ stretching frequencies of $\left.\mathrm{CpRe}\left({ }^{13} \mathrm{CO}\right)_{x}\left({ }^{12} \mathrm{CO}\right)_{3-\mathrm{x}}\right)$. Unfortunately, this 
$2015 \mathrm{~cm}^{-1}$ band is superimposed on one of the parent $\mathrm{CpRe}\left({ }^{12} \mathrm{CO}\right)_{3} \mathrm{v}=1 \rightarrow 2$ transition. Therefore, instead of showing an instantaneous depletion followed by a rapid recovery, it bleaches gradually to a constant level (Figure 5.7c).

The vibrationally hot bands described above unavoidably impede an accurate description of bands that are important in determining the reaction dynamics. The $1990 \mathrm{~cm}^{-1}$ band of the final product $\mathbf{F}$ overlaps with the $\mathrm{v}=2$ hot band (at $\sim 1900 \mathrm{~cm}^{-1}$ ) of the $\mathrm{CpRe}\left({ }^{13} \mathrm{CO}\right)(\mathrm{CO})_{2}$ bleach at $2015 \mathrm{~cm}^{-1}$. As a result, the kinetic trace of $\mathbf{F}$ measured at $1990 \mathrm{~cm}^{-1}$ exhibits a sharp rise due to the hot band, then decays to a constant level (Figure 5.7). To derive the underlying product dynamics, the kinetic traces measured at $1990 \mathrm{~cm}^{-1}, 2004 \mathrm{~cm}^{-1}$, and $2015 \mathrm{~cm}^{-1}$ were fit to a model which takes into account the bleach recovery, product formation, and vibrational relaxation (see 5.5). The extracted formation time for the product is $4.4 \pm 2.6$ ps. Typically, the initial solvation of metal carbonyls occurs within 1-2 ps following photolysis of one CO ligand [98], from which one can infer the time scale for a barrierless associative reaction in solution. Accordingly, our results suggest that solvation of the $\eta^{5}-\mathrm{CpRe}(\mathrm{CO})_{2}$ through the $\mathrm{Si}-\mathrm{H}$ bond of the solvent leads directly to the formation of the product.

The kinetics of $\mathbf{G}$ measured at $1887 \mathrm{~cm}^{-1}$ show an exponential rise of $2.5 \pm 1.6 \mathrm{ps}$ and remain at the same intensity to $\sim 1 \mathrm{~ns}$ (Figure 5.5). As indicated by the absence of peaks at 1887 and $1948 \mathrm{~cm}^{-1}$ in the static FTIR spectrum at the bottom panel of Figure 5.5 , this alkyl solvate must eventually react further to form the final product. To investigate the long-time behavior of this chemical system, we also performed nanosecond step-scan FTIR experiments. Representative kinetics of the alkyl-solvate (G) and the product $(\mathbf{F})$ are shown in Figure 5.8. The kinetics of the product $\mathbf{F}$ measured at $1990 \mathrm{~cm}^{-1}$ show an instrument-limited rise and an exponential growth of $6.8 \pm 0.2 \mu \mathrm{s}$. The alkyl solvate recorded at $1887 \mathrm{~cm}^{-1}$ shows a $7.2 \pm 0.2 \mu$ s exponential decay to a constant level. This finite absorbance is attributed to the $\mathrm{CO}$ stretch of 
$\mathrm{CpRe}\left({ }^{13} \mathrm{CO}\right)\left({ }^{12} \mathrm{CO}\right)(\mathrm{H})\left(\mathrm{SiEt}_{3}\right)$, estimated to be at $1892 \mathrm{~cm}^{-1}$, the kinetics of which should be similar to those of the product.

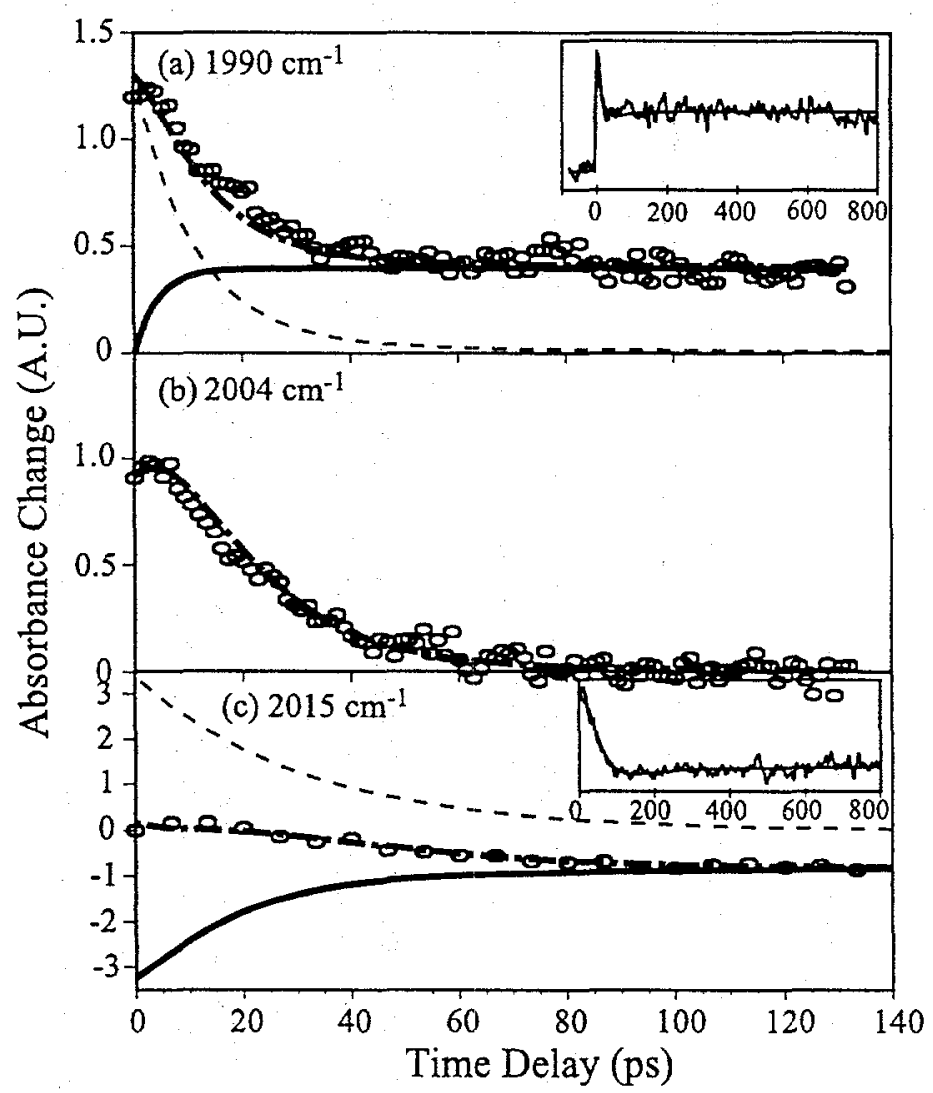

Figure 5.7 Modeling of the observed transient traces of $\mathrm{CpRe}(\mathrm{CO})_{3}$.

(a) The transient trace recorded at the $1990-\mathrm{cm}^{-1}$ product band (open circles). The solid line is the deconvolved product formation kinetics and the dashed line is the contribution from vibrationally excited $\mathrm{CpRe}\left({ }^{12} \mathrm{CO}\right)_{2}\left({ }^{13} \mathrm{CO}\right)$. The observed kinetic trace is obtained (the dash-dot line) by re-convolving these two traces. Insert: A long scan at the same wavelength, showing that the amplitude of this peak remains the same up to $\sim 1 \mathrm{~ns}$. (b) The transient trace (open circles) of the $2004 \mathrm{~cm}^{-1}$ band, which is attributed to vibrationally excited $\mathrm{CpRe}\left({ }^{12} \mathrm{CO}\right)_{2}\left({ }^{13} \mathrm{CO}\right)$. The dash-dot line follows the model presented in 5.5. (c) The transient trace recorded at the $2015 \mathrm{~cm}^{-1}$ parent bleach (open circles) due to photolysis of the naturally abundant $\mathrm{CpRe}\left({ }^{12} \mathrm{CO}\right)_{2}\left({ }^{13} \mathrm{CO}\right)$. The solid line is the deconvolved bleach recovery and the dashed line is the reconstructed kinetics from the vibrationally excited $\mathrm{CpRe}\left({ }^{12} \mathrm{CO}\right)_{3}$. 
The alkyl-solvate $\mathbf{G}$ therefore displays a slightly longer decay time than the rise time of F. Similar to the treatment performed in the $\mathrm{CpMn}(\mathrm{CO})_{3} / \mathrm{Et}_{3} \mathrm{SiH}$ system, the relative probability of the initial solvation through the ethyl group and the $\mathrm{Si}-\mathrm{H}$ bond is estimated to be $75 \%$ and $25 \%$, respectively. Assuming that transition-state theory is applicable, the measured rise time of the product gives an estimate of the free energy of the reaction barrier to be $\Delta G^{+}=10.41 \pm 0.02 \mathrm{kcal} / \mathrm{mol}$ (Equation 5.2). Thus the apparent ratedetermining step is shown to be the dissociative rearrangement of the initial alkyl-solvate, similar to the manganese reaction.

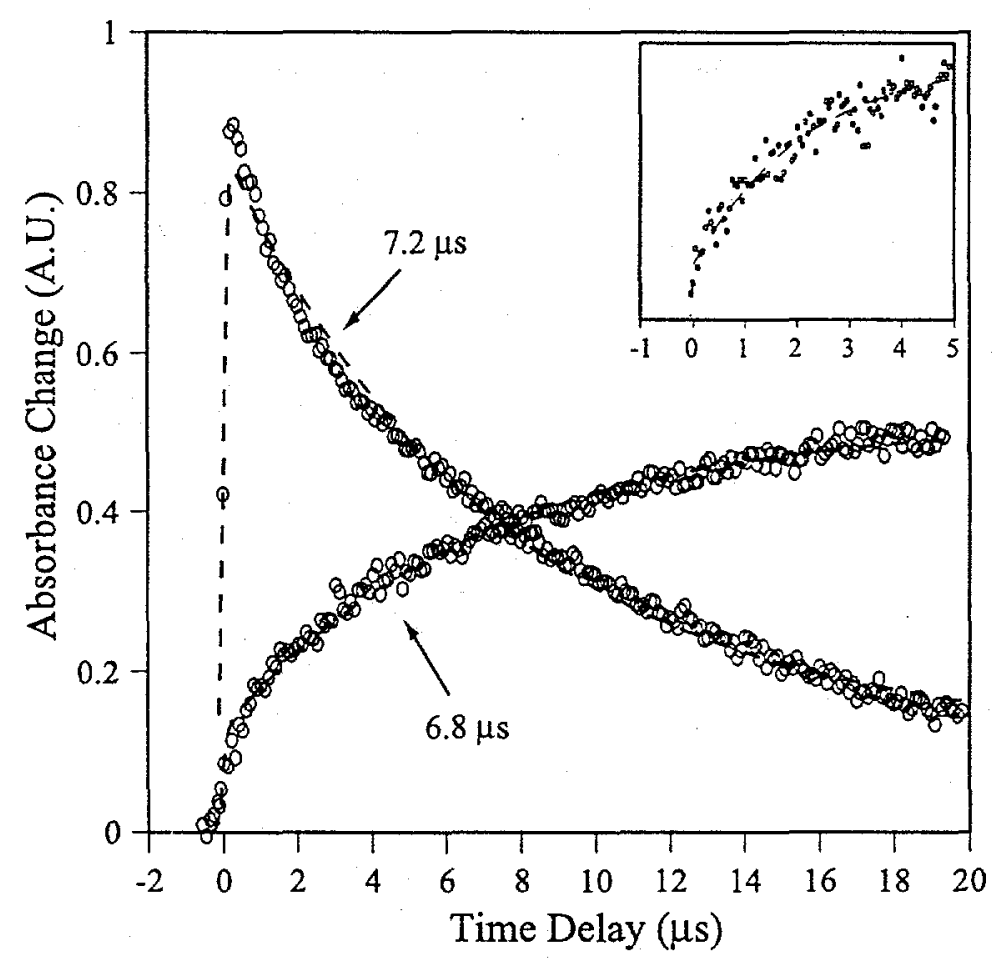

Figure 5.8 Nanosecond kinetics of $\mathrm{CpRe}(\mathrm{CO})_{3}$ following 295-nm excitation.

Nanosecond kinetics (open circles) of $\mathrm{CpRe}(\mathrm{CO})_{3}$ in neat triethylsilane after 295-nm UV photolysis at (F) $1990 \mathrm{~cm}^{-1}$, the CO stretch of the silyl adduct CpRe(CO) $2(\mathrm{H})\left(\mathrm{SiEt}_{3}\right) ;(\mathrm{G})$ $1948 \mathrm{~cm}^{-1}$, the CO stretch of the alkyl solvate $\mathrm{CpRe}(\mathrm{CO})_{2}\left(\mathrm{Et}_{3} \mathrm{SiH}\right)$. The time constants for the exponential fits (dashed lines) are shown in the graph. Insert: A short scan at $1990 \mathrm{~cm}^{-1}$ showing the instrument-limited rise due to formation of product $F$ on the ultrafast time scale. 


\subsection{3 ab initio Calculation Results}

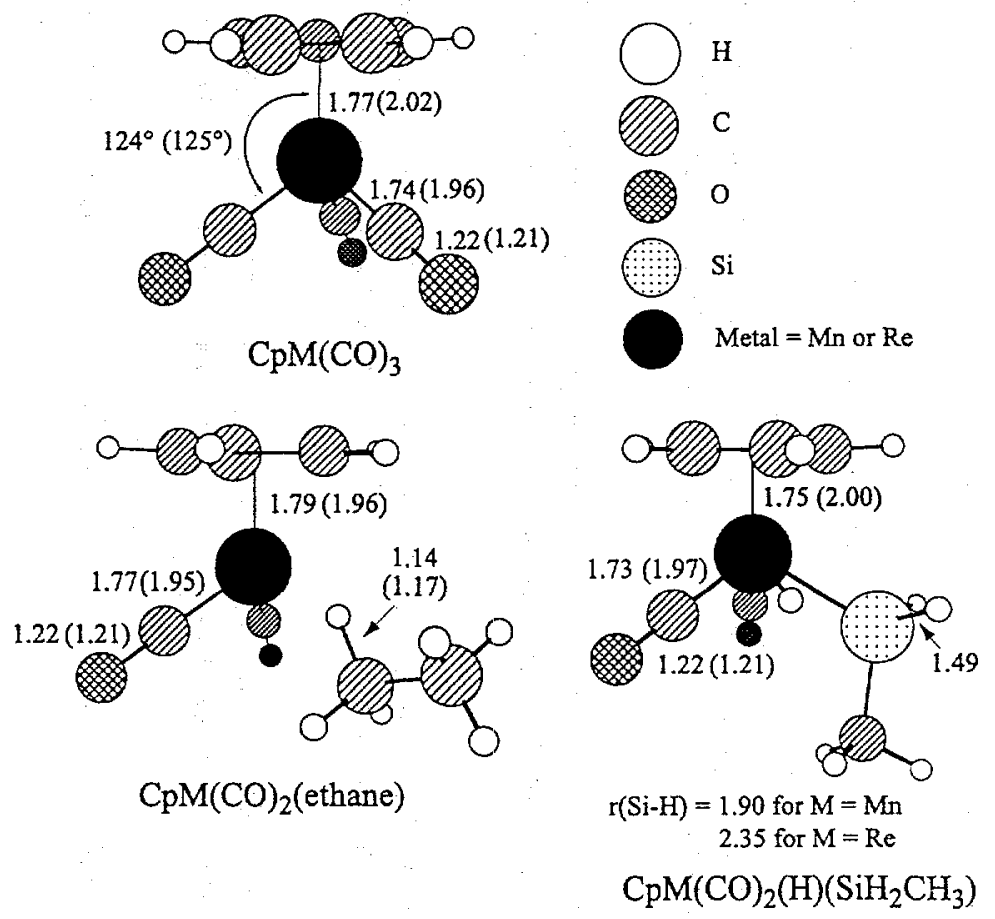

Figure 5.9 Geometries of the parent molecules, the ethane-solvate, and the final product optimized at the MP2 / lanl2dz level of theory.

The bond lengths are in $\AA$ and angles in degrees. Parameters for the rhenium complexes are in parenthesis.

Geometry optimization. Figure 5.9 shows the geometries of the parent molecules $\eta^{5}-\mathrm{CpM}(\mathrm{CO})_{3}[121]$, the dicarbonyl metal complexes solvated by an ethane molecule $\eta^{5}-\mathrm{CpM}(\mathrm{CO})_{2}$ (ethane), and the final products $\eta^{5}-\mathrm{CpM}(\mathrm{CO})_{2}(\mathrm{H})\left(\mathrm{SiH}_{2} \mathrm{CH}_{3}\right)$; all optimized at the MP2/lanl2dz level of theory. As displayed in Table 5.1, the metal-Cp and metal$\mathrm{CO}$ distances for the parent molecules calculated at the MP2 level agree reasonably well with the experimental values, except for the slightly overestimated $\mathrm{C}-\mathrm{O}$ bond lengths. Qualitatively, the metal-ligand distances should reflect the relative sizes of the manganese and the rhenium atoms. As displayed in Table 5.1, the rhenium complexes exhibit consistently a longer metal-ligand distance by $\sim 0.17 \AA$ over the manganese complexes, indicating a larger diameter for a complexed Re atom over a Mn atom. 
Table 5.1. Critical bond distances (in $\AA$ ) of the important structures for the $\mathrm{Si}-\mathrm{H}$ bond activation reactions optimized at the MP2 / lanl2dz level of theory. ${ }^{a}$

\begin{tabular}{lllllll}
\hline compound & $\mathrm{Cp}-\mathrm{M}$ & $\mathrm{M}-\mathrm{CO}$ & $\mathrm{C}-\mathrm{O}$ & $\mathrm{M}-\mathrm{H}$ & $\begin{array}{l}\mathrm{M}-\mathrm{Si} \\
(\mathrm{M}-\mathrm{C})\end{array}$ & $\begin{array}{l}\mathrm{S} i-\mathrm{H} \\
(\mathrm{C}-\mathrm{H})\end{array}$ \\
\hline$\eta^{5}-\mathrm{CpMn}(\mathrm{CO})_{3}$ & $1.77(1.77)^{\mathrm{b}}$ & $1.74(1.78)^{\mathrm{b}}$ & $1.22(1.16)^{\mathrm{b}}$ & & & \\
singlet $\eta^{5}-\mathrm{CpMn}(\mathrm{CO})_{2}$ & $1.80\{1.86\}$ & $1.78\{1.79\}$ & $1.21\{1.19\}$ & & & \\
triplet $\eta^{5}-\mathrm{CpMn}(\mathrm{CO})_{2}$ & $1.84\{1.94\}$ & $1.82\{1.83\}$ & $1.19\{1.18\}$ & & & \\
$\eta^{5}-\mathrm{CpMn}(\mathrm{CO})_{2}\left(\mathrm{H}_{3} \mathrm{CCH} \mathrm{C}_{3}\right)$ & 1.79 & 1.77 & 1.22 & 1.77 & 2.73 & 1.14 \\
$\eta^{5}-\mathrm{CpMn}(\mathrm{CO})_{2}(\mathrm{H})\left(\mathrm{H}_{2} \mathrm{SiCH}_{3}\right)$ & 1.75 & 1.73 & 1.22 & 1.51 & 2.44 & 1.90 \\
$\eta^{5}-\mathrm{CpRe}(\mathrm{CO})_{3}$ & $2.02(1.96)^{\mathrm{c}}$ & $1.96(1.90)^{\mathrm{c}}$ & $1.21(1.17)^{\mathrm{c}}$ & & & \\
singlet $\eta^{5}-\mathrm{CpRe}(\mathrm{CO})_{2}$ & $1.94\{1.97\}$ & $1.95\{1.89\}$ & $1.22\{1.19\}$ & & & \\
triplet $\eta^{5}-\mathrm{CpRe}(\mathrm{CO})_{2}$ & $2.03\{2.04\}$ & $1.98\{1.91\}$ & $1.19\{1.19\}$ & & & \\
$\eta^{5}-\mathrm{CpRe}(\mathrm{CO})_{2}\left(\mathrm{H}_{3} \mathrm{CCH}_{3}\right)$ & 1.96 & 1.95 & 1.21 & 1.89 & 2.84 & 1.17 \\
$\eta^{5}-\mathrm{CpRe}(\mathrm{CO})_{2}(\mathrm{H})\left(\mathrm{H}_{2} \mathrm{SiCH}_{3}\right)$ & 2.00 & 1.97 & 1.21 & 1.69 & 2.57 & 2.35 \\
\hline
\end{tabular}

${ }^{a}$ Numbers in () parenthesis are experimental values, and those in curvy brackets are from DFT calculations.

${ }^{b}$ Fitzpatrick, P. J.; Le Page, Y.; Sedman, J.; Butler, I. S., Inorg. Chem. 1981, $20,2852$.

${ }^{c}$ Fitzpatrick, P. J.; Le Page, Y.; Sedman, J.; Butler, I. S., Acta Crystallogr., Sect B, 1981, B37, 1052.

For the ethane dicarbonyl complexes in Figure 5.9, the ethane molecule interacts with the metal center via one of its $\mathrm{C}-\mathrm{H}$ bonds through an $\eta^{2}$ interaction. The bond lengths for the coupling C-H bond are 1.14 and $1.17 \AA$ for the $\mathrm{Mn}$ and Re compounds, respectively. These calculated $\mathrm{C}-\mathrm{H}$ bond lengths, compared to $1.10 \AA$ for a typical $\mathrm{C}-\mathrm{H}$ bond in a saturated alkane from the same level of calculation, show a significantly extended $\mathrm{C}-\mathrm{H}$ bond $(+0.07 \AA)$ when complexed to the Re center but only slight $(+0.04$ $\AA$ ) extension when coupled to the Mn complex. The more extended $\mathrm{C}-\mathrm{H}$ bond in the $\mathrm{Re}$ case suggests that the $\mathrm{Re}$ atom interacts more strongly with a $\mathrm{C}-\mathrm{H}$ bond, hence forming a more stable alkyl complex. Finally, the relative extent of the $\mathrm{Si}-\mathrm{H}$ bond activation by the $\mathrm{Mn}$ and $\mathrm{Re}$ compounds is clearly demonstrated by the $\mathrm{Si}-\mathrm{H}$ bond distances in the final products. The calculated $\mathrm{Si}-\mathrm{H}$ bond lengths for the $\mathrm{Mn}$ and $\mathrm{Re}$ complexes are respectively 1.90 and $2.35 \AA$ compared to a typical silane $\mathrm{Si}-\mathrm{H}$ bond length of $1.49 \AA$. Apparently, the degree of bond-breakage can be inferred from the product $\mathrm{Si}-\mathrm{H}$ bond lengths. In the hydridosilyl manganese product, the $\mathrm{Si}-\mathrm{H}$ bond is $0.41 \AA$ longer than a $\mathrm{Si}-\mathrm{H}$ bond in an isolated silane. The hydridosilyl rhenium product, on the other hand, 
exhibits a much longer distance $(0.86 \AA$ longer than an isolated $\mathrm{Si}-\mathrm{H}$ bond) between the silicon and the hydrogen atom, marking a completely broken $\mathrm{Si}-\mathrm{H}$ bond.

Side View
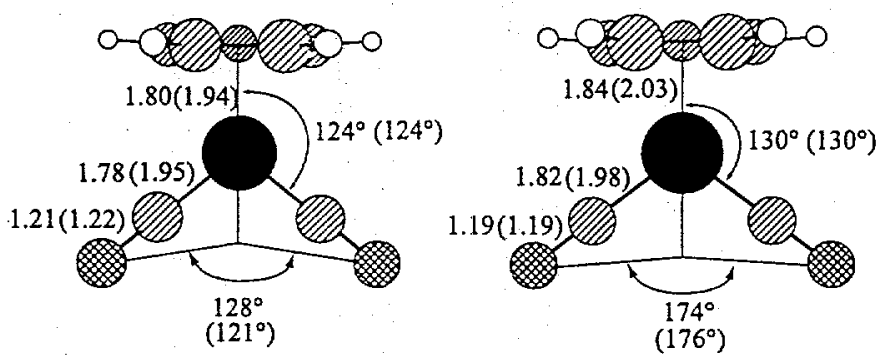

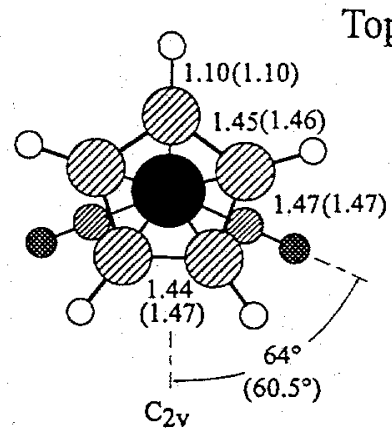

singlet $\mathrm{CpM}(\mathrm{CO})_{2}$

Top View

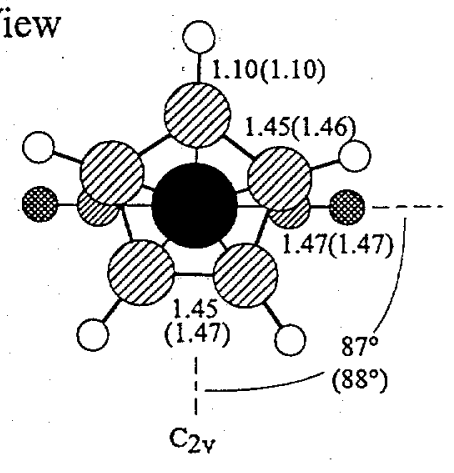

triplet $\mathrm{CpM}(\mathrm{CO})_{2}$

Figure 5.10 Side view and top view for the MP2/lanl2dz optimized geometries of the 16-e species.

The bond lengths are in $\AA$ and angles in degrees. Parameters for the rhenium complexes are in parenthesis.

Shown in Figure 5.10 are the geometries for the $16-\mathrm{e}^{-}$species $\eta^{5}-\mathrm{CpM}(\mathrm{CO})_{2}$ in their singlet and triplet states. Despite the similarity of the structures, one notices a difference in the OC-M-CO angle. Due to the larger radius of the Re atom and hence reduced ligand-ligand repulsive interaction, the angle is $93.47^{\circ}$ for the $\operatorname{Re}$ complex, compared to $95.11^{\circ}$ for the $\mathrm{Mn}$ one; or when projected onto the $\mathrm{Cp}$ plane, $121^{\circ}$ for the $\mathrm{Re}$ and $128^{\circ}$ for the Mn species. 
For both metal complexes, the geometries for the singlet species are quite different from the triplet ones; most notable are the positions of the $\mathrm{M}-\mathrm{CO}$ bond relative to the molecular $\mathrm{C}_{2 \mathrm{v}}$ plane. For example, the angle between the $\mathrm{M}-\mathrm{CO}$ bond and the molecular $\mathrm{C}_{2 \mathrm{~V}}$ plane for the singlet state $\eta^{5}-\mathrm{CpMn}(\mathrm{CO})_{2}$ is $64^{\circ}$, but is $87^{\circ}$ for the triplet state. In addition, the $\mathrm{M}-\mathrm{Cp}$ and $\mathrm{M}-\mathrm{CO}$ distances of the triplet species are longer than those of the singlet ones. Also, the $\mathrm{CO}$ bond lengths are shorter in the triplet states compared to those of the singlet states. Such a decrease in the metal-CO interaction in the triplet state has also been observed in Siegbahn's calculations of $\mathrm{CpM}(\mathrm{CO}), \mathrm{M}=\mathrm{Co}$, Rh, and Ir.[106] Also shown in Table 5.1 are the DFT results for the $16-\mathrm{e}^{-}$species which are in general agreement with those from the MP2 calculations. The DFT-calculated angle between the $\mathrm{M}-\mathrm{CO}$ bond and the molecular $\mathrm{C}_{2 \mathrm{v}}$ plane for the singlet state $\eta^{5}-\mathrm{CpMn}(\mathrm{CO})_{2}$ is $63^{\circ}$, and is $84^{\circ}$ for the triplet state. Analogous parameters for the singlet and triplet rhenium complexes are $58^{\circ}$ and $88^{\circ}$, respectively. The C-O bond lengths predicted by DFT methods, however, are consistently shorter than those predicted by MP2 methods. The $\mathrm{Cp}-\mathrm{Mn}$ distance from DFT calculations is significantly longer than that from UMP2 methods (by $\sim 0.1 \AA$ ), perhaps due to the inadequacy of the UMP2 wavefunctions for describing high-spin species. These geometric parameters are summarized in Table 5.1. Several attempts to optimize $\eta^{3}-\mathrm{CpMn}(\mathrm{CO})_{2}, \quad \eta^{3}-\mathrm{CpMn}(\mathrm{CO})_{2}\left(\mathrm{CH}_{4}\right), \quad$ or $\eta^{3}-\mathrm{CpMn}(\mathrm{CO})_{2}\left(\mathrm{SiH}_{4}\right)$ were unsuccessful, however, as an $\eta^{5}-\mathrm{CpMn}(\mathrm{CO})_{2}$ configuration was invariably obtained, regardless of the initial guess.

Energy calculations. The calculated metal-ligand interaction energies are listed in Table 5.2. After BSSE and ZPE corrections, the binding energies between the Mn complex and the $\mathrm{CO}$ ligand or the $\mathrm{Si}-\mathrm{H}$ bond appear to be in good agreement with the experimental values. Compared to the $\mathrm{Mn}$ complex, the Re complex shows a binding energy to the $\mathrm{CO}$ ligand which is stronger by $5.81 \mathrm{kcal} / \mathrm{mol}$. As a result, there is comparatively less excess energy available to the Re metal fragment after photolysis. Also, the hydridosilyl rhenium product is thermodynamically more stable than the manganese product by a marked $11.98 \mathrm{kcal} / \mathrm{mol}$. In the case of an alkane ligand, it has been found experimentally 
$[122,123]$ and theoretically [124] that the interaction energy between a transition metal and a series of chain alkanes may increase with alkane chain length. Consequently, in the present calculation which uses ethane as the complexed solvent to model the solvation of $\eta^{5}-\mathrm{CpM}(\mathrm{CO})_{2}$ through the ethyl group of $\mathrm{Et}_{3} \mathrm{SiH}$, the complexation energies obtained are likely to be underestimated. Nonetheless, the calculated binding energies of the ethane with $\mathrm{Mn}$ and $\mathrm{Re}$ complexes allow a qualitative understanding of the relative stability of $\eta^{5}-\mathrm{CpMn}(\mathrm{CO})_{2}$ (alkane) and $\eta^{5}-\mathrm{CpRe}(\mathrm{CO})_{2}$ (alkane). Our results show that an ethane molecule binds more strongly to the Re complex than to the Mn complex by about 3.5 $\mathrm{kcal} / \mathrm{mol}$. Similar stability for the $\eta^{5}-\mathrm{CpRe}(\mathrm{CO})_{2} \mathrm{~L}(\mathrm{~L}=n$-heptane, $\mathrm{Xe}$, and $\mathrm{Kr})$ has also been reported in a recent paper by Sun et: al. [125]. Consequently, if the free-energy barrier of a dissociative rearrangement is dominated by the enthalpy of complexation, it would take longer for an $\eta^{5}-\mathrm{CpRe}(\mathrm{CO})_{2}\left(\mathrm{Et}_{3} \mathrm{SiH}\right)$ to rearrange dissociatively to allow interaction of a $\mathrm{Si}-\mathrm{H}$ bond with the metal center, in comparison with the manganese system. Our experimental results show that it takes $6.8 \mu$ s for the rhenium compound to undergo such a rearrangement whereas only 177 ns are required for the analogous process involving the manganese compound; or equivalently a $2.16 \mathrm{kcal} / \mathrm{mol}$ difference in their free-energy barrier.

Table 5.2. Calculated MP2 / lanl2dz binding energies (in $\mathrm{kcal} / \mathrm{mol}$ ) for singlet $\eta^{5}-\mathrm{CpM}(\mathrm{CO})_{2} \mathrm{~L}, \mathrm{M}=\mathrm{Mn}, \mathrm{Re}^{\mathrm{a}}$

\begin{tabular}{llllllll}
\hline & \multicolumn{3}{c}{$\mathrm{Mn}$} & & \multicolumn{3}{c}{$\mathrm{Re}$} \\
\cline { 2 - 4 } \cline { 6 - 8 } $\mathrm{L}$ & $\mathrm{ZPE}$ & $\Delta \mathrm{E}$ & $\Delta \mathrm{E}(\mathrm{BSSE})$ & & $\mathrm{ZPE}$ & $\Delta \mathrm{E}$ & $\Delta \mathrm{E}(\mathrm{BSSE})$ \\
\hline $\mathrm{CO}$ & $-2.26^{\mathrm{b}}$ & 95.55 & $51.76(55)^{\mathrm{c}}$ & & $-2.75^{\mathrm{b}}$ & 97.87 & 57.57 \\
$\mathrm{Et}$ & $0.21^{\mathrm{d}}$ & 16.21 & $2.91(10)^{\mathrm{e}}$ & & $0.21^{\mathrm{d}}$ & 20.56 & 6.41 \\
$\mathrm{SiH}_{3} \mathrm{CH}_{3}$ & $111.93^{\mathrm{d}}$ & 53.83 & $22.92(24.4)^{\mathrm{e}}$ & & $109.62^{\mathrm{d}}$ & 71.88 & 34.90 \\
\hline
\end{tabular}

${ }^{a}$ Experimental values are in parentheses. ${ }^{b}$ Calculated using HF frequencies. ${ }^{\circ}$ Angelici, R. J.; Loewen, W. Inorg. Chem. 1967, 6, 682. ${ }^{\mathrm{d}}$ Calculated using MP2 frequencies. ${ }^{\mathrm{e}}$ Burkey, T. J. J. Am. Chem. Soc. 1990, 112,8329 .

Table 5.3. Comparison of the energy difference, $\Delta \mathrm{E}=\mathrm{E}$ (singlet)-E(triplet), between the singlet and triplet $\eta^{5}$ - $\mathrm{CpM}(\mathrm{CO})_{2}$ calculated using different methods (in $\mathrm{kcal} / \mathrm{mol}$ ). 


\begin{tabular}{lll}
\hline & $\mathrm{Mn}$ & $\mathrm{Re}$ \\
\hline MP2 $^{\mathrm{a}}$ & -10.93 & -5.63 \\
DFT $^{\mathrm{b}}$ & 8.09 & -5.62 \\
CASSCF-PT2 $^{\mathrm{b}}$ & 9.86 & -7.88 \\
\hline
\end{tabular}

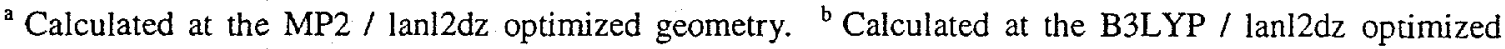
geometry.

We next turn to the relative energies of the singlet and the triplet $\eta^{5}-\mathrm{CpMn}(\mathrm{CO})_{2}$. The DFT results show a lower energy for the triplet $\eta^{5}-\mathrm{CpMn}(\mathrm{CO})_{2}$ relative to the singlet $(\Delta \mathrm{E}=8.09 \mathrm{kcal} / \mathrm{mol})$, in contrast to the $\mathrm{MP} 2$ results which predict a lower-energy singlet state by $10.93 \mathrm{kcal} / \mathrm{mol}$. These conflicting results are probably due to the near degeneracy of the singlet and the triplet states. Similar inconsistent results from different calculation methods have also been reported in the literature [106]. For $\mathrm{CpCo}(\mathrm{CO})$, Siegbahn found that the MP2 method gave a singlet ground state, whereas other methods including DFT / B3LYP and CASPT2 consistently yield a triplet ground state. To minimize the near-degeneracy effect, CAS MCSCF-PT2 calculations were performed at the DFT geometries; and the results predict a ground triplet state for $\eta^{5}-\mathrm{CpMn}(\mathrm{CO})_{2}, 9.86$ $\mathrm{kcal} / \mathrm{mol}$ lower than the singlet state. Therefore, our calculations are in favor of a triplet ground state. For the rhenium complexes, analogous calculations consistently predict a singlet ground state for $\eta^{5}-\mathrm{CpRe}(\mathrm{CO})_{2}$. These results are summarized in Table 5.3. Finally, numerical CO stretching frequencies at DFT level were calculated for the singlet $\eta^{5}-\mathrm{CpMn}(\mathrm{CO})_{2}$ (methane) $\left\{1889\right.$ and $\left.1946 \mathrm{~cm}^{-1}\right\}$ and for the triplet $\eta^{5}-\mathrm{CpMn}(\mathrm{CO})_{2}\{1870$ and $1967 \mathrm{~cm}^{-1}$. These results will be used in the assignment of $\mathbf{C}$ in next section. ${ }^{1}$

\subsection{Discussion}

1 The frequencies for a triplet dicarbonyl were determined on the $\eta^{5}-\mathrm{CpMn}(\mathrm{CO})_{2}$ instead of $\eta^{5}-\mathrm{CpMn}(\mathrm{CO})_{2}$ (alkane) because it is only weakly solvated by alkanes, to be discussed in the next section. 


\subsubsection{Activation of the Silicon-Hydrogen Bond of $\mathrm{Et}_{3} \mathrm{SiH}$ by $\mathrm{CpMn}(\mathrm{CO})_{3}$}

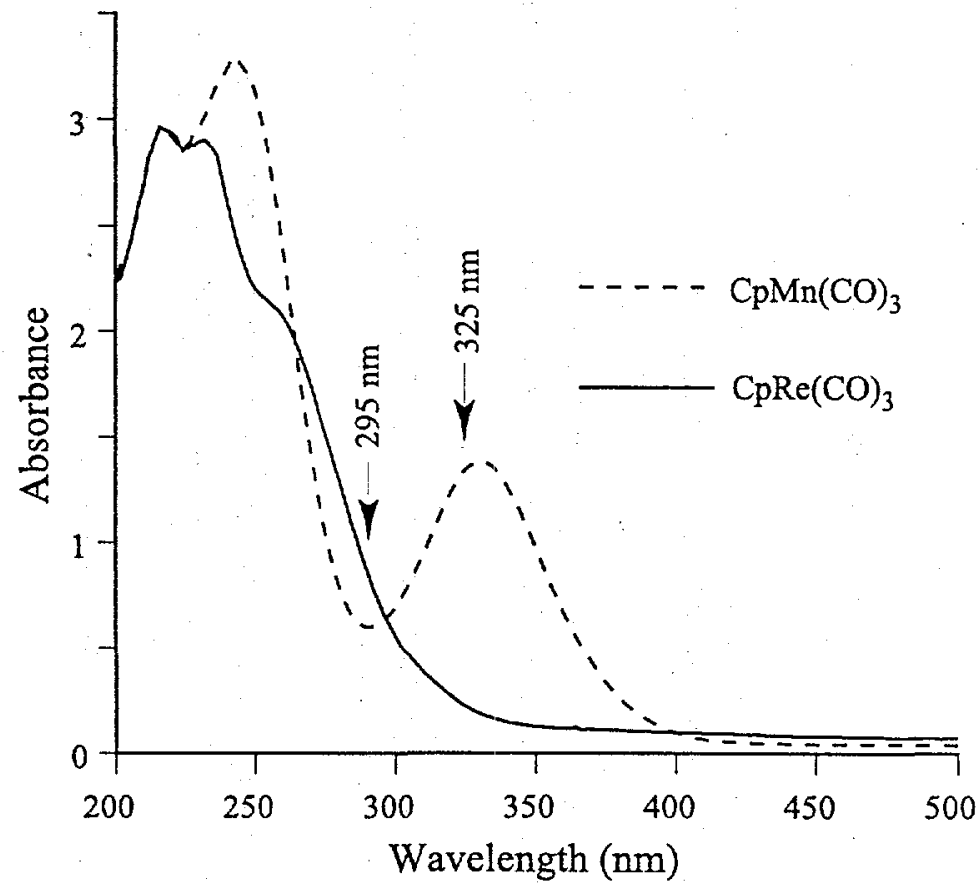

Figure 5.11 UV/ Vis spectra of $\mathrm{CpMn}(\mathrm{CO})_{3}$ and $\mathrm{CpRe}(\mathrm{CO})_{3}$.

$\mathrm{UV} / \mathrm{Vis}$ spectra of the $\mathrm{CpMn}(\mathrm{CO})_{3}$ and $\mathrm{CpRe}(\mathrm{CO})_{3}$ in neat triethylsilane taken under experimental condition. Excitation wavelengths are indicated by arrows.

Effects of excitation wavelength. In the case of 295-nm excitation (Paper I), the parent molecule $\eta^{5}-\mathrm{CpMn}(\mathrm{CO})_{3}$ loses one $\mathrm{CO}$ ligand to produce two dicarbonyls (designated as $\mathbf{D}^{*}$ and $\mathbf{C}$ in Figure 5.13), the former is quickly solvated to give $\mathbf{D}$. The intermediate $\mathbf{C}$ then converts to form $\mathbf{D}$ on a time scale of $90 \mathrm{ps}$, or to $\mathbf{B}$ in $\sim 71 \mathrm{ps.} \mathrm{The} \mathrm{branching} \mathrm{ratio}$ in generating these two intermediates at this excitation wavelength, calculated in Paper I from the kinetic trace of $\mathbf{D}$, is approximately $1: 1$. Since this pump energy resides in the trough of two electronic states (see Figure 5.11), it is conceivable that the $\mathbf{D}^{*}$ and $\mathbf{C}$ may be related to the two electronically excited states. To examine how the excited states affect the reaction mechanism, we changed the excitation wavelength to the lower-energy band at $325 \mathrm{~nm}$ in the current study. Compared to excitation at $295 \mathrm{~nm}$, the $325-\mathrm{nm}$ excitation light injects less energy to the chemical system and samples different regions of the excited potential energy surface (PES). As a result, it may lead to dissociation 
channels that have different product energy distribution. Indeed, the kinetics of $\mathbf{C}$ and $\mathbf{D}$ following 325-nm excitation show little, if any, excess vibrational energy. This is to be compared with results from $295-\mathrm{nm}$ excitation, which indicate that $\sim 23$ ps is required to dissipate excess vibrational energy. For clarity, the kinetic traces of $\mathrm{D}$ recorded at the two pump energies are reproduced in Figure 5.12. Accessing a different part of the excited PES also changes the branching ratio from $A \rightarrow D^{*} \rightarrow D: A \rightarrow C \rightarrow D \approx 1: 1(295-n m$ excitation) to $1: 3(325 \mathrm{~nm})$. This may imply that the lower-energy band at $\sim 330 \mathrm{~nm}$ correlates with the dissociation channel that leads to $\mathrm{C}$, and the higher-energy band to $\mathrm{D}$.

The nature of the intermediate $C$. In Paper I, two possible candidates for the observed transient intermediate $C$ were proposed: a ring-slipped $\eta^{3}-\mathrm{CpMn}(\mathrm{CO})_{2}$ or $\eta^{5}-\mathrm{CpMn}(\mathrm{CO})_{2}$ in another electronic state, presumably a triplet state. The $\eta^{3}-\mathrm{CpMn}(\mathrm{CO})_{2}$ species $[75$, 126, 127], however, has only 14 valence electrons for the $\mathrm{Mn}-\mathrm{a}$ first-row transition metal-severely violating the $18-\mathrm{e}^{*}$ rule. Our theoretical modelings also support the energetic instability of $\eta^{3}$ complexes. $C$ is therefore unlikely to be a ring-slipped 14-especies. Another possible assignment for $\mathrm{C}$ is that it is a dicarbonyl in the triplet ground state. There have been precedents for $16-\mathrm{e}^{-}$organometallics having a triplet ground state [128-131], the geometry of which may differ from that of the singlet state to allow a different ligand vibrational frequency $[107,132]$. Our calculations suggest a triplet ground state for $\eta^{5}-\mathrm{CpMn}(\mathrm{CO})_{2}$. We next compare the theoretical $\mathrm{CO}$ stretching frequencies to the experimental results. Recent developments in $a b$ initio methods have shown promising results in identifying organometallic species through frequency calculations $[78,133,134]$. The DFT calculations for the triplet dicarbonyl show a +21 $\mathrm{cm}^{-1}$ shift of the higher-energy CO band and a $-19 \mathrm{~cm}^{-1}$ shift of the lower-energy band of the solvated singlet dicarbonyl, compatible with experimental observation of frequency shifts of $\mathbf{C}$ relative to $\mathbf{D}$. Therefore, it seems reasonable to attribute $\mathbf{C}$ to a triplet $\eta^{5}-\mathrm{CpMn}(\mathrm{CO})_{2}$. 


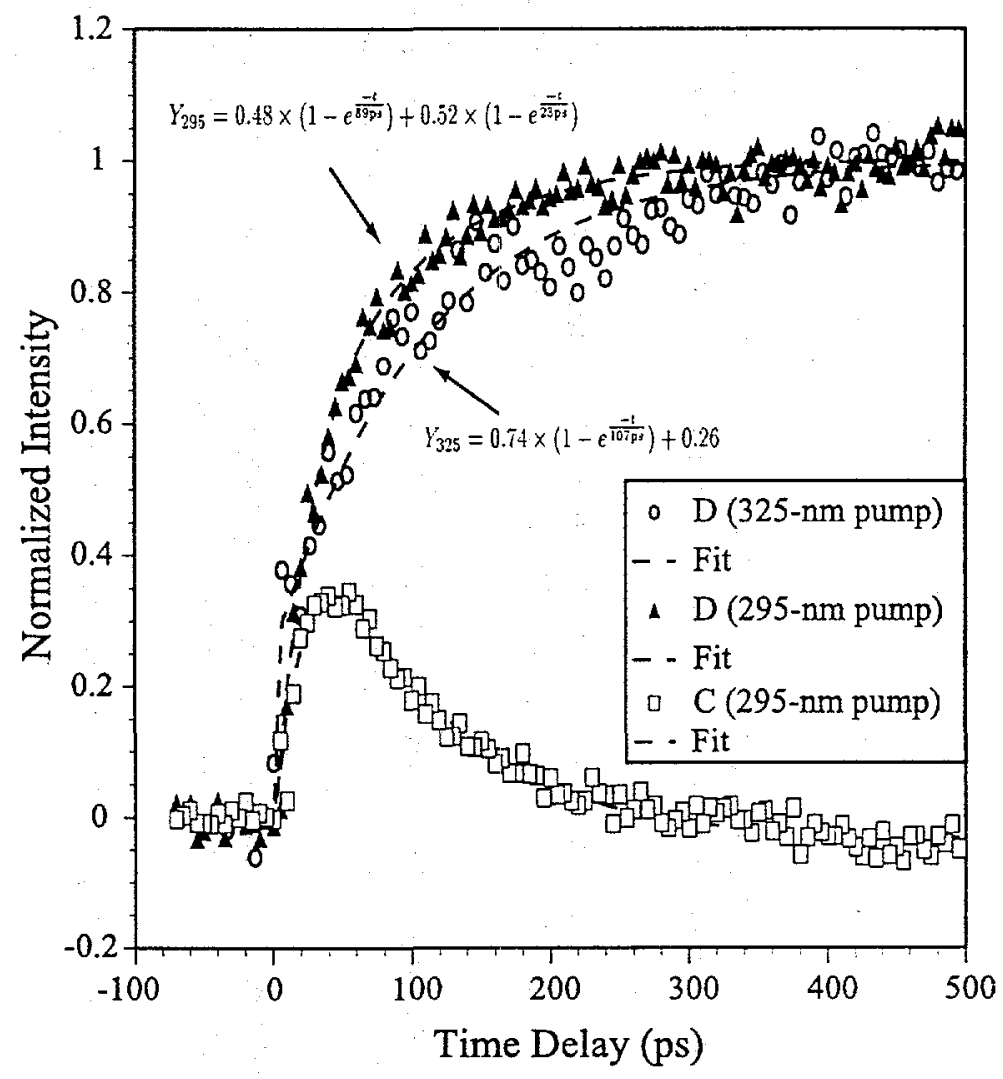

Figure 5.12 Comparison of ultrafast kinetics of $\mathrm{CpMn}(\mathrm{CO})_{3}$ at different pump wavelengths.

Ultrafast kinetic traces of $\mathbf{D}$ (filled triangles), $\mathbf{C}$ (open squares) following 295-nm excitation, and $\mathbf{D}$ (open circles) following $325-\mathrm{nm}$ excitation. Also shown are the functions $Y_{295}$ and $Y_{325}$ that are used to model the two normalized traces of $\mathbf{D}$. Corresponding fits are shown as dashed lines. Clearly, the relative intensity of $\mathbf{C}$ to $\mathbf{D}$ is lower when excited at $295 \mathrm{~nm}$ compared to that of $325-\mathrm{nm}$ excitation in Figure 5.3. This wavelength dependence can be quantified by comparing the fitting coefficients of $Y_{295}$ and $\mathrm{Y}_{325}$. Please see text for details.

Solvation of the triplet and singlet manganese dicarbonyls. In the context of $\mathrm{C}$ being a triplet state $\eta^{5}-\mathrm{CpMn}(\mathrm{CO})_{2}$, we next discuss the interaction of $\mathbf{C}$ and the solvent. Unlike the singlet $16-\mathrm{e}^{-}$species which can be solvated readily by alkanes and rare-gas atoms ( $\mathrm{Kr}, \mathrm{Xe})$, it has been suggested theoretically that transition metals in their triplet state interact only weakly with these solvents [135-137]. This conclusion has been used to explain the high reactivity of triplet $\eta^{5}-\mathrm{CpCo}(\mathrm{CO})$ towards a free $\mathrm{CO}$ in liquidified 
rare-gas solution or in alkane solution [128]: In that case, the incoming CO ligand does not require much activation energy to displace an alkane or rare-gas molecule which couples weakly to the metal center of $\eta^{5}-\mathrm{CpCo}(\mathrm{CO})$. Triplet-to-singlet intersystem crossing in chemical systems such as triplet $\eta^{5}-\mathrm{CpCo}(\mathrm{CO})$ or triplet $\eta^{5}-\mathrm{CpMn}(\mathrm{CO})_{2}$ is further complicated by the fact that the complex of interest is always under the influence of solvent molecules in a liquid environment. It was found by Heilweil et. al. that triplet $\eta^{5}-\mathrm{CpCo}(\mathrm{CO})$ reacts very quickly (< vibrational cooling time) with the strongly-binding ligand 1 -hexene to form presumably a singlet $\pi$-complex, in which a $l$-hexene molecule complexes to the Co metal through its $\mathrm{C}=\mathrm{C}$ double bond [120]. This is to be compared with the lifetime $(\sim \mathrm{ms})$ of triplet $\eta^{5}-\mathrm{CpCo}(\mathrm{CO})$ in weakly-binding solvents such as $\mathrm{Kr}$ and $\mathrm{Xe}$ [128]. Hence the surrounding solvent molecules, especially those interacting strongly with the metal center, need to be considered in describing the reaction coordinate for spin-crossover and solvent exchange. In other words, the interconversion from triplet $\mathbf{C}$ to singlet $\mathbf{D}$ should probably be considered as a concerted spin-crossover / solvation process.

With this picture in mind, the solvation of the initially photo-generated triplet and singlet dicarbonyls can be described as follows. Probably due to steric hindrance and statistical favor of the ethyl groups over the $\mathrm{Si}-\mathrm{H}$ bond, the nascent singlet $\eta^{5}-\mathrm{CpMn}(\mathrm{CO})_{2}$ is preferentially solvated via the ethyl moiety of $\mathrm{Et}_{3} \mathrm{SiH}$ to form $\mathrm{D}$. The solvation of the triplet dicarbonyl, however, may be quite different. Assuming that a triplet $\eta^{5}-\mathrm{CpMn}(\mathrm{CO})_{2}$ interacts initially with the ethyl moiety of an $\mathrm{Et}_{3} \mathrm{SiH}$ molecule, the interaction between the alkyl group and a triplet $\mathrm{Mn}$ is so weak that the surrounding solvent molecules have sufficient thermal energy to easily reorient or displace the interacting $\mathrm{Et}_{3} \mathrm{SiH}$, until the triplet $\mathrm{Mn}$ encounters a strongly-coupling $\mathrm{Si}-\mathrm{H}$ site. This process is expected to be on the order of a few picoseconds, the time scale for typical solvent motions. It would thus appear that a triplet transition metal complex is preferentially solvated by the more strongly-coupling sites of a solvent molecule. Similarly a preferential solvation has also been observed in Heilweil's experiments on 
$\mathrm{CpCo}(\mathrm{CO})$ in neat $l$-hexene, where one would expect to see solvation of $\mathrm{CpCo}(\mathrm{CO})$ through both the alkyl chain and the $\mathrm{C}=\mathrm{C}$ bond of $l$-hexene. The only product observed, however, was the strongly bound $\pi$-complex. Our data show that the product kinetics exhibit only one exponential rise on the ultrafast time scale, which is perhaps due to the preferential initial solvation of the singlet $\eta^{5}-\mathrm{CpMn}(\mathrm{CO})_{2}$ via the ethyl group and the triplet $\eta^{5}-\mathrm{CpMn}(\mathrm{CO})_{2}$ through the stronger-binding $\mathrm{Si}-\mathrm{H}$ bond of the solvent molecule.

The reaction mechanism. In the light of the above discussion, the photo-chemical Si-H bond activation reaction of $\eta^{5}-\mathrm{CpMn}(\mathrm{CO})_{3}$ in neat $\mathrm{Et}_{3} \mathrm{SiH}$ under the ambient conditions is summarized in Figure 5.13. We found that there are two excited states involved in the experimentally accessible region. The higher-energy UV band is correlated with the generation of the dicarbonyl $\eta^{5}-\mathrm{CpMn}(\mathrm{CO})_{2}\left(\mathbf{D}^{*}\right)$ in its electronic singlet manifold, while the lower-energy band is correlated to the triplet $\eta^{5}-\mathrm{CpMn}(\mathrm{CO})_{2}(\mathrm{C})$. Following previous discussion on the preferential solvation of $\mathbf{D}^{*}$ through the ethyl moiety and that $84 \%$ of the total product comes from $\mathbf{D}$, the branching ratio for $\mathbf{A} \rightarrow \mathbf{D}^{*}$ to $\mathbf{A} \rightarrow \mathbf{C}$ can be calculated to be $42 \%: 58 \%$ at 295 -nm excitation. The initial solvation of these two intermediates via the $\mathrm{Si}-\mathrm{H}$ bond or the ethyl moiety of the solvent partitions the reaction into two pathways with a branching ratio close to $1: 5.3$ (16\% through the $\mathrm{Si}-\mathrm{H}$ bond and $84 \%$ through the ethyl group). Solvation of $\mathbf{C}$ via the $\mathrm{Si}-\mathrm{H}$ bond leads to the final product $\mathbf{B}$ on a time scale of $\sim 100 \mathrm{ps}$, possibly through a concerted coordination/spin-crossover process. Solvation of $\mathbf{D}^{*}$ through the ethyl group of the solvent molecule results in $\mathrm{D}$, whose population is augmented by interconversion from the triplet $\mathbf{C}$ on a similar time scale of $\sim 100$ ps. Finally, the ethyl-solvate reacts to form the final product $\mathbf{B}$ through a dissociative rearrangement on a time scale of $\sim 177 \mathrm{~ns}$, corresponding to a barrier height of $\Delta \mathrm{G}^{+} \approx 8.25 \mathrm{kcal} / \mathrm{mol}$. 


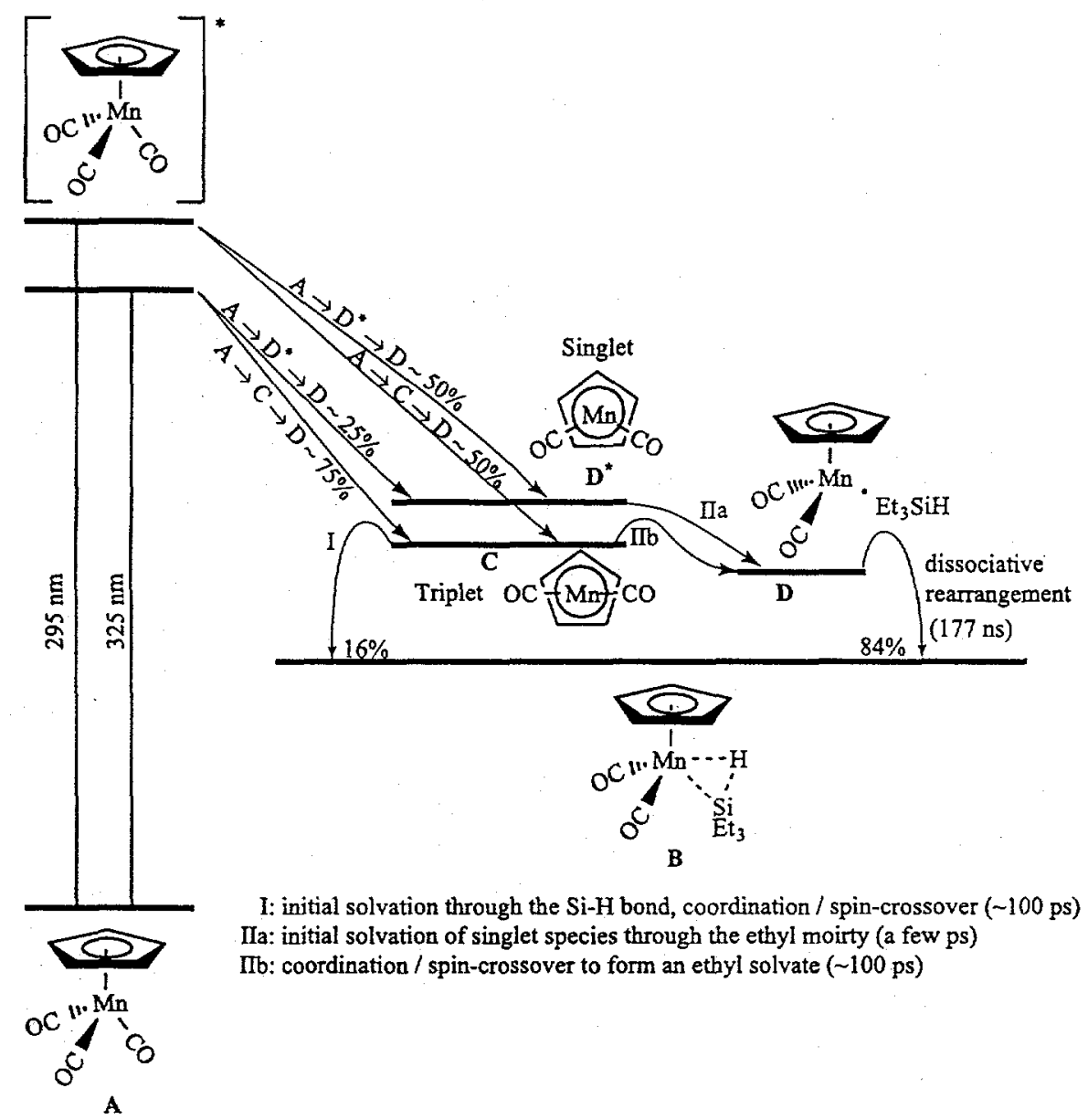

Figure 5.13 Proposed reaction mechanism for the silicon-hydrogen bond activation by $\mathrm{CpMn}(\mathrm{CO})_{3}$ covering the ultrafast dynamics to nanosecond kinetics.

The nascent singlet $\eta^{5}-\mathrm{CpMn}(\mathrm{CO})_{2}$, denoted $\mathbf{D}^{*}$, is also shown for the purpose of discussion. See text for details. 


\subsubsection{Activation of the Silicon-Hydrogen Bond of $\mathrm{Et}_{3} \mathrm{SiH}$ by $\mathrm{CpRe}(\mathrm{CO})_{3}$}

In comparison to the manganese compound, the photo-chemical reaction of $\eta^{5}-\mathrm{CpRe}(\mathrm{CO})_{3}$ (E) with $\mathrm{Et}_{3} \mathrm{SiH}$ is much simpler (see Figure 5.14). Photoly'sis of $\mathbf{E}$ results in a dicarbonyl species, $\eta^{5}-\mathrm{CpRe}(\mathrm{CO})_{2}\left(\mathrm{G}^{*}\right)$, in its singlet electronic ground state. Subsequent solvation of $\mathbf{G}^{*}$ partitions the reaction into two courses to the formation of the final product. Interaction of $\mathrm{G}^{*}$ with the $\mathrm{Si}-\mathrm{H}$ bond of the solvent molecule leads directly to the final product $\eta^{5}-\mathrm{CpRe}(\mathrm{CO})_{2}(\mathrm{H})\left(\mathrm{SiEt}_{3}\right), \mathbf{F}$, with a time constant of $\sim 4.4 \mathrm{ps}$. Another pathway proceeds through solvation of $\mathbf{G}^{*}$ via the ethyl group of the solvent to form $\eta^{5}-\mathrm{Cp} \operatorname{Re}(\mathrm{CO})_{2}\left(\mathrm{Et}_{3} \mathrm{SiH}\right), \mathbf{G}$, on a time scale of $\sim 2.5 \mathrm{ps}$, and further reacts to form the final product $\mathbf{F}$. The relative probability of (solvation through the ethyl moiety)/(solvation through the $\mathrm{Si}-\mathrm{H}$ bond). for the rhenium complex is found to be approximately 3 , compared to 5.3 for the manganese complex. The difference may be attributed to steric interaction from the ligands. The smaller radius of the Mn center may cause increased steric interaction between the ligands and the solvent molecule's ethyl group when solvation proceeds through the $\mathrm{Si}-\mathrm{H}$ bond. Such steric hindrance is reduced when solvation occurs through the ethyl moiety. The larger diameter of the Re center makes it more accessible to chemically different sites of a solvent molecule, resulting in a branching ratio of approximately 3 -the ratio of three ethyl groups to one $\mathrm{Si}-\mathrm{H}$ bond in an $\mathrm{Et}_{3} \mathrm{SiH}$ molecule. 


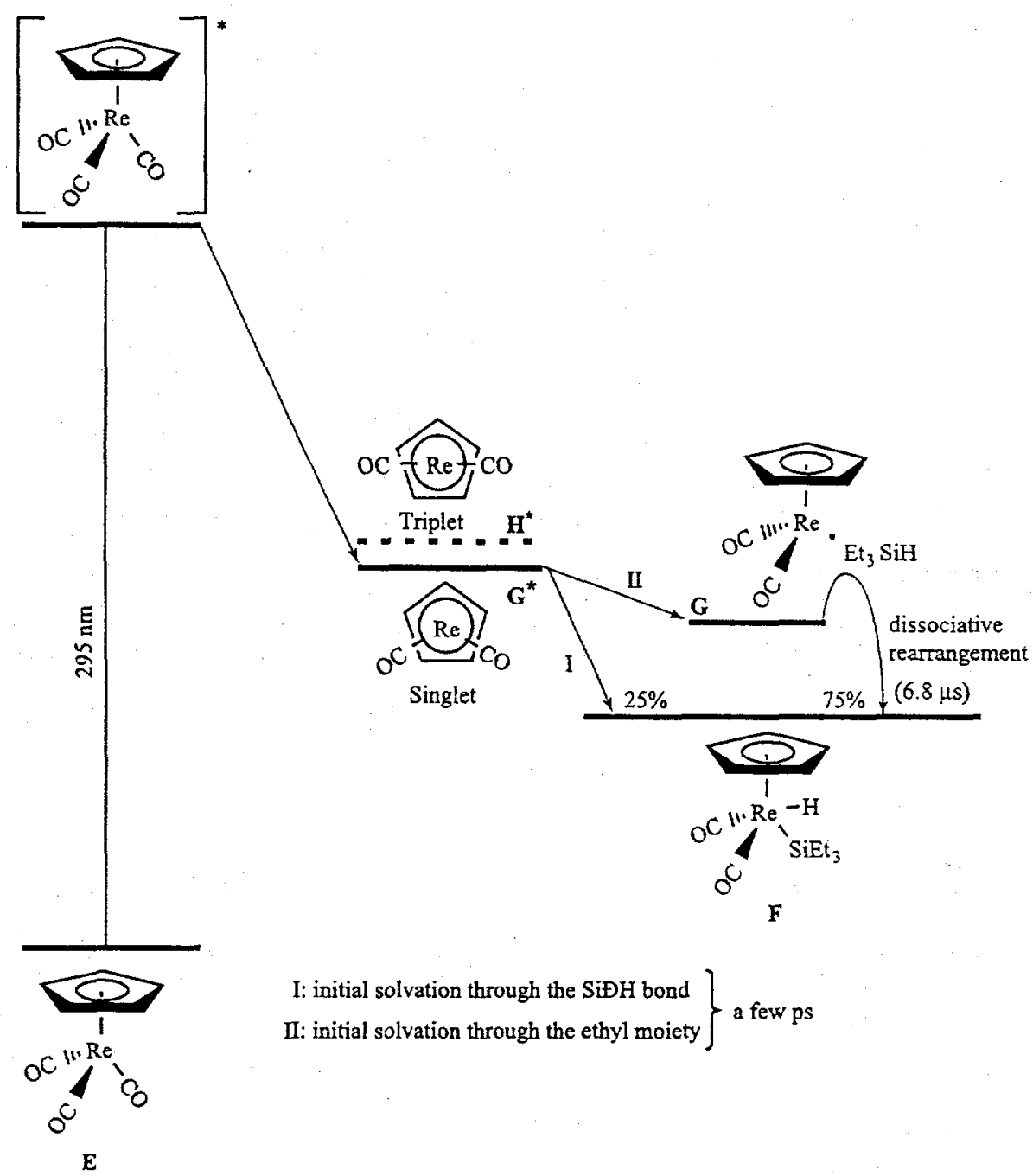

Figure 5.14 Proposed reaction mechanism for the silicon-hydrogen bond activation by $\mathrm{CpRe}(\mathrm{CO})_{3}$ covering the ultrafast dynamics to microsecond kinetics.

The nascent singlet and triplet $\eta^{5}-\mathrm{CpRe}(\mathrm{CO})_{2}$, denoted $\mathbf{H}^{*}$ and $\mathbf{G}^{*}$, are also shown for the purpose of discussion. See text for details. 


\subsection{Closing Remarks}

Due to the very complicated dynamic processes in a liquid environment, photochemical reactions such as the bond-activation reactions studied here may span several orders of magnitude in time-from hundreds of femtosecond for dissociating a chemical bond to the time scale governed by a diffusional process or an energetic barrier. Combining the use of the femtosecond and nanosecond UV pump-IR probe spectroscopy, the elementary reaction steps including changes in molecular conformation and electronic multiplicity may be studied by monitoring the time-evolution of the reactive intermediates. The nature of the intermediates whose lifetimes were too short for conventional spectroscopic characterization were studied using first-principle quantumchemical methods. Utilizing these powerful tools, it is also possible to assess the relative probability of parallel, dynamically partitioned reaction channels. We have investigated photo-chemical $\mathrm{Si}-\mathrm{H}$ bond activation reaction by group VIIB, $\mathrm{d}^{6}$ transition metal complexes $\eta^{5}-\mathrm{CpM}(\mathrm{CO})_{3}(\mathrm{M}=\mathrm{Mn}, \mathrm{Re})$. The detailed reaction mechanisms are summarized in Figure 5.13 and Figure 5.14.

Despite the fact that $\mathrm{Mn}$ and Re belong to the same group in the periodic table, we have shown in this paper that the reaction dynamics of $\eta^{5}-\mathrm{CpMn}(\mathrm{CO})_{3}$ or $\eta^{5}-\mathrm{CpRe}(\mathrm{CO})_{3}$ with $\mathrm{Et}_{3} \mathrm{SiH}$ is very different on the ultrafast time scale and depends on the nature of electronic structure of the molecule in question. If more than one dissociation channel (excited state) are involved in the initial photolysis as in the Mn case, which has two experimentally accessible UV bands, the reaction may dynamically partition into several parallel pathways leading to different transient intermediates. The nature of these transient species have been investigated using $a b$ initio calculations. The calculation results provided strong evidence that photolysis of the parent $\eta^{5}-\mathrm{CpMn}(\mathrm{CO})_{3}$ generates both singlet and triplet $\eta^{5}-\mathrm{CpMn}(\mathrm{CO})_{2}$ species, which accounts for the experimental 
observations consistently. The correlation of these reaction pathways to the excited states were studied by examination of their branching ratios, which were determined from ultrafast kinetic traces of the intermediates, at different excitation wavelengths. In the case of $\mathrm{Mn}$, the higher-energy band was found to associate with formation of singlet $\eta^{5}-\mathrm{CpMn}(\mathrm{CO})_{2}$ while the lower-energy band with that of triplet $\eta^{5}-\mathrm{CpM}(\mathrm{CO})_{2}$. Interaction of solvent molecules with the singlet and triplet $\mathrm{Mn}$ complexes were also discussed.

The metal dicarbonyls $\eta^{5}-\mathrm{CpMn}(\mathrm{CO})_{2}$ or $\eta^{5}-\mathrm{CpRe}(\mathrm{CO})_{2}$ resulting from UV photolysis of a $\mathrm{CO}$ ligand were found to be solvated by a solvent molecule in a few. picoseconds. From this point on, both metal complexes share a common reaction pattern that solvation through chemically different sites of a solvent molecule further partitions the reaction into two routes. The final product could form on the ultrafast regime when the coordinatively unsaturated 16- $\mathrm{e}^{-}$metal complex interacted with the $\mathrm{Si}-\mathrm{H}$ bond of the solvent molecule. On longer time scales, both reactions ( $\mathrm{Mn}$ and $\mathrm{Re}$ ) were shown to proceed through a kinetically favored intermediate-a metal dicarbonyl solvated by the ethyl moiety of solvent molecule. The branching ratio for these solvation-partitioned reaction pathways, derived from the product kinetic traces taken at ns or $\mu$ s time scales, was argued to be governed by steric interaction. The apparent rate-determining step was found to originate from dissociative rearrangement from this ethyl-solvate to the thermodynamically more stable hydrido-silyl product. The associated free-energy: barriers were also determined and were in excellent agreement with results from previous low-temperature studies (for $\mathrm{Mn}$ ).

Through detailed mechanistic study, we were able to experimentally determine the $\mathrm{Si}-\mathrm{H}$ bond-breaking step. The time scale for such a process in a liquid environment was for the first time measured to be $4.4 \mathrm{ps}$. The result allows an upper bound for the time scale of the $\mathrm{Si}-\mathrm{H}$ bond-activation step, confirming $a b$ initio theoretical predictions $[8,87]$. This is in sharp contrast to the analogous $\mathrm{C}-\mathrm{H}$ bond-cleaving process which 
takes approximately $230 \mathrm{~ns}$. In this case, the $8.3 \mathrm{kcal} / \mathrm{mol}$ energy barrier corresponding to cleaving an alkane $\mathrm{C}-\mathrm{H}$ bond has been attributed to poor spatial overlap and energy match of the molecular orbitals between an alkane $\mathrm{C}-\mathrm{H}$ bond and a transition metal center; the hydrogen atom and an alkyl group have to separate before forming chemical bonds with the metal center $[8,87]$. Extension of the current work, including a close interplay between experiment and theory, to other transition metals reacting with different chemical bonds are expected to provide a better understanding about the chemistry of bond-cleavage reactions.

\subsection{Supplementary}

In the ultrafast experiment of $\eta^{5}-\mathrm{CpRe}(\mathrm{CO})_{3} / \mathrm{Et}_{3} \mathrm{SiH}$, several events that occur concurrently on the $\sim 100$ ps time scale have complicated the transient spectra in the frequency region from $1990 \mathrm{~cm}^{-1}$ to $2030 \mathrm{~cm}^{-1}$. These events include the recovery of the parent $\eta^{5}-\mathrm{CpRe}\left({ }^{12} \mathrm{CO}\right)_{3}$ bleach due to re-equilibration of the vibrational population, bleach recovery of $\eta^{5}-\mathrm{CpRe}\left({ }^{12} \mathrm{CO}\right)_{2}\left({ }^{13} \mathrm{CO}\right)$, and the formation of the product $\eta^{5}-\mathrm{CpRe}(\mathrm{CO})_{2}(\mathrm{H})\left(\mathrm{SiEt}_{3}\right)$. Summarized below are these processes and their associated frequencies:

$1990 \mathrm{~cm}^{-1}$ : $\quad$ product formation, vibrational relaxation of ${ }^{13} \mathrm{v}=2$.

$2004 \mathrm{~cm}^{-1}$ : vibrational relaxation of ${ }^{13} \mathrm{v}=1$.

$2015 \mathrm{~cm}^{-1}$ : bleach recovery of ${ }^{13} \mathrm{v}=0$, vibrational relaxation of ${ }^{12} \mathrm{v}=1$.

$2030 \mathrm{~cm}^{-1}$ : bleach recovery of ${ }^{12} \mathrm{v}=0$.

where superscripts ${ }^{12}$ and ${ }^{13}$ refer respectively to the parent molecule $\eta^{5}-\mathrm{CpRe}\left({ }^{12} \mathrm{CO}\right)_{3}$ and the naturally abundant ${ }^{13} \mathrm{CO}$ mono-substituted $\eta^{5}-\mathrm{CpRe}\left({ }^{13} \mathrm{CO}\right)\left({ }^{12} \mathrm{CO}\right)_{2}$. To deconvolve the dynamics of individual events out of the complicated spectra, we first consider the population of the $i^{\text {th }}$ vibrational state of $\eta^{5}-\mathrm{CpRe}\left({ }^{13} \mathrm{CO}\right)\left({ }^{12} \mathrm{CO}\right)_{2}$. Assuming that the 
relaxation rate of the $i$ state to the $(i-1)$ state is proportional to the population $n_{i}$, and that $n_{i}$ is replenished only by relaxation from the $(i+1)$ state, we can write the coupled rate equations for vibrational states $i=0,1$, and 2 as

\section{Equation 5.3}

$$
\frac{d^{13} n_{i}}{d t}=-{ }^{13} k_{i, i-1}{ }^{13} n_{i}+{ }^{13} k_{i+1, i}{ }^{13} n_{i+1}
$$

${ }^{13} k_{i, i-1}$

where the $k_{i, j}$ are the rate constants for the transition from $i$ state to $j$ state. Similarly, the population dynamics for $\mathrm{CO}$ vibrational states $i=0$ and 1 of $\eta^{5}-\mathrm{CpRe}\left({ }^{12} \mathrm{CO}\right)_{3}$ can be expressed as

Equation 5.4

$$
\frac{d^{12} n_{0}}{d t}={ }^{12} k_{1.0}^{12} n_{1}
$$

Equation 5.5

$$
\frac{d^{12} n_{1}}{d t}=-{ }^{12} k_{1,0}^{12} n_{1}
$$

Finally, the formation of the final product at $1990 \mathrm{~cm}^{-1}$ is assumed to be represented by a single exponential,

\section{Equation 5.6}

$$
I_{\text {product }}=C_{\text {product }}\left(1-e^{-t / \tau_{\text {product }}}\right)
$$

We further assume that the IR absorbance of state $i$, denoted by $I_{i}$, is proportional to its population $n_{i}$. The observed kinetic traces can then be fit to this model by solving the coupled differential equations numerically and collecting terms contributing to the relevant frequencies. The optimized parameters are: ${ }^{13} I^{0}{ }_{3}=0.29 \pm 0.15,{ }^{13} I^{0}{ }_{2}=1.30 \pm$ $0.015,{ }^{13} I_{1}{ }_{1}=0.92 \pm 0.070,{ }^{13} I_{0}{ }_{0}=-3.24 \pm 0.16,{ }^{13} k_{3,2}=0.083 \pm 0.0036 \mathrm{ps}^{-1},{ }^{13} k_{2,1}=$ $0.088 \pm 0.0076 \mathrm{ps}^{-1},{ }^{13} k_{1,0}=0.088 \pm 0.015 \mathrm{ps}^{-1},{ }^{12} k_{1,0}=0.032 \pm 0.0025 \mathrm{ps}^{-1}$, and $\tau_{\text {product }}=$ $4.35 \pm 2.6$ ps. The deconvolved kinetic traces are displayed in Figure 5.7 and explained in the figure caption. Note that the intensities at $t=0$ of state $i$, denoted by $I_{i}^{0}$, does not reflect the initial population on each vibrational state since we do not have a priori knowledge of the extinction coefficient for each vibrational state. 


\section{Femtosecond Infrared Studies of a Prototypical One- Electron Oxmative-Addition Reaction: Chlorine ATOM Abstraction by the Re(CO) $)_{5}$ Radical.}

\subsection{Introduction}

Oxidative-addition is one class of fundamental reactions of organometallic complexes. A comprehensive understanding of the mechanism requires knowledge of the dynamics of all the intermediates. The extremely fast reaction rates, however, have made it experimentally challenging to elucidate the reaction scheme. Femtosecond inirared (fsIR) spectroscopy, which is capable of "real-time" observation and characterization of the reactive intermediates down to hundreds of femtosecond, offers the possibility of deducing the elementary steps of a photochemical reaction in room-temperature solutions. Recently, this technique has been successfully used to unravel the reaction dynamics on a time scale faster than that of diffusion in the photo-initiated two-electron oxidative-addition reactions of $\mathrm{C}-\mathrm{H}$ and $\mathrm{Si}-\mathrm{H}$ bond activation $[15-18,80]$. This communication reports the use of fs-IR spectroscopy to study a prototypical one-electron oxidative-addition. The transition-metal complex under study is the $17-\mathrm{e}^{-}(\mathrm{CO})_{5} \mathrm{Re}$ radical, generated by photo-dissociation of the $\operatorname{Re}-\operatorname{Re}$ bond of $\operatorname{Re}_{2}(C O)_{10}$. The $17-e^{-}$ radical reacts to extract a $\mathrm{Cl}$ atom from a chlorinated methane solvent. The reaction may proceed through a strongly-solvated $19-\mathrm{e}^{-}$intermediate, through a charge-transfer intermediate, or through a weakly-solvated $17-\mathrm{e}^{-}$intermediate as shown in Figure 6.1 [911]: 


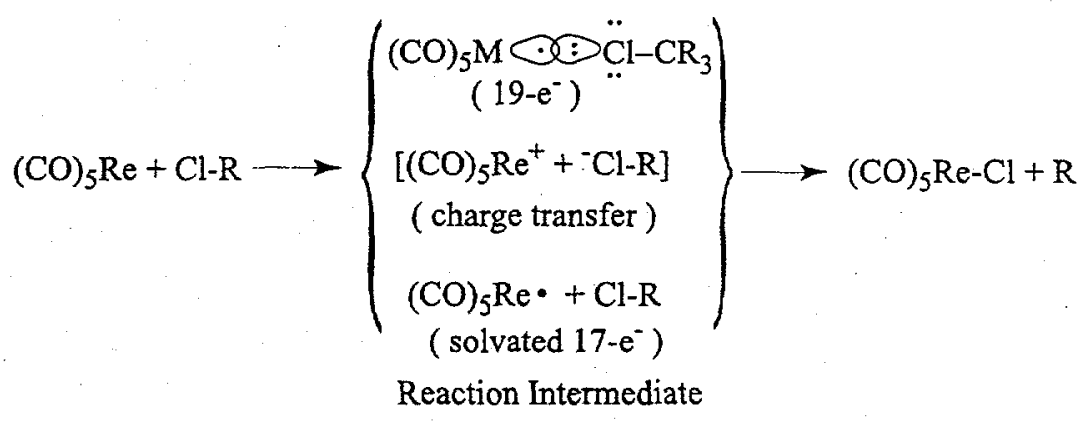

Figure 6.1 Previously proposed reaction schemes for $\mathrm{Cl}$ atom abstraction by $\operatorname{Re}(\mathrm{CO})_{5}$.

This aspect is examined directly for the first time by following the reaction from initiation to completion with $300-\mathrm{fs}$ time resolution. To study the nature of the reaction barrier, the rates are measured along a series of chlorinated methane solutions under ambient conditions. The transition states are studied using density-functional theoretical (DFT) methods.

\subsection{Results and Discussion}

As shown in the static FTIR in Figure 6.2d, the final product $(\mathrm{CO})_{5} \mathrm{ReCl}$ in neat $\mathrm{CCl}_{4}$ solution exhibits two $\mathrm{CO}$ stretching peaks at 1982 and $2045 \mathrm{~cm}^{-1}$ [138]. At shorter time delays $\left(40 \mathrm{~ns}<2.5 \mu \mathrm{s}\right.$ ) in Figure 6.2c, ${ }^{1}$ there appear five additional bands at 1945 , 1985, 1998, 2005, and $2055 \mathrm{~cm}^{-1}$ that are assigned to the equatorially solvated nonacarbonyl species $e q-\operatorname{Re}_{2}(\mathrm{CO})_{9}\left(\mathrm{CCl}_{4}\right)$, in agreement with low-temperature studies [139]. On the hundreds of picosecond time scale (Figure 6.2b), one sees a broad feature centering at around $1990 \mathrm{~cm}^{-1}$, marked by a down-pointing arrow. This spectrum is to be compared with that taken in the chemically inert hexanes solution (Figure 6.2a), which also exhibits a broad band centering at around $1992 \mathrm{~cm}^{-1}$, assigned to the weakly solvated $\operatorname{Re}(\mathrm{CO})_{5}$ radical in hexanes solution [140]. It follows that the broad feature at

\footnotetext{
${ }^{1}$ Please refer to the supplementary section of this chapter for technical details. All uncertainties reported in herein represent one standard deviation.
} 


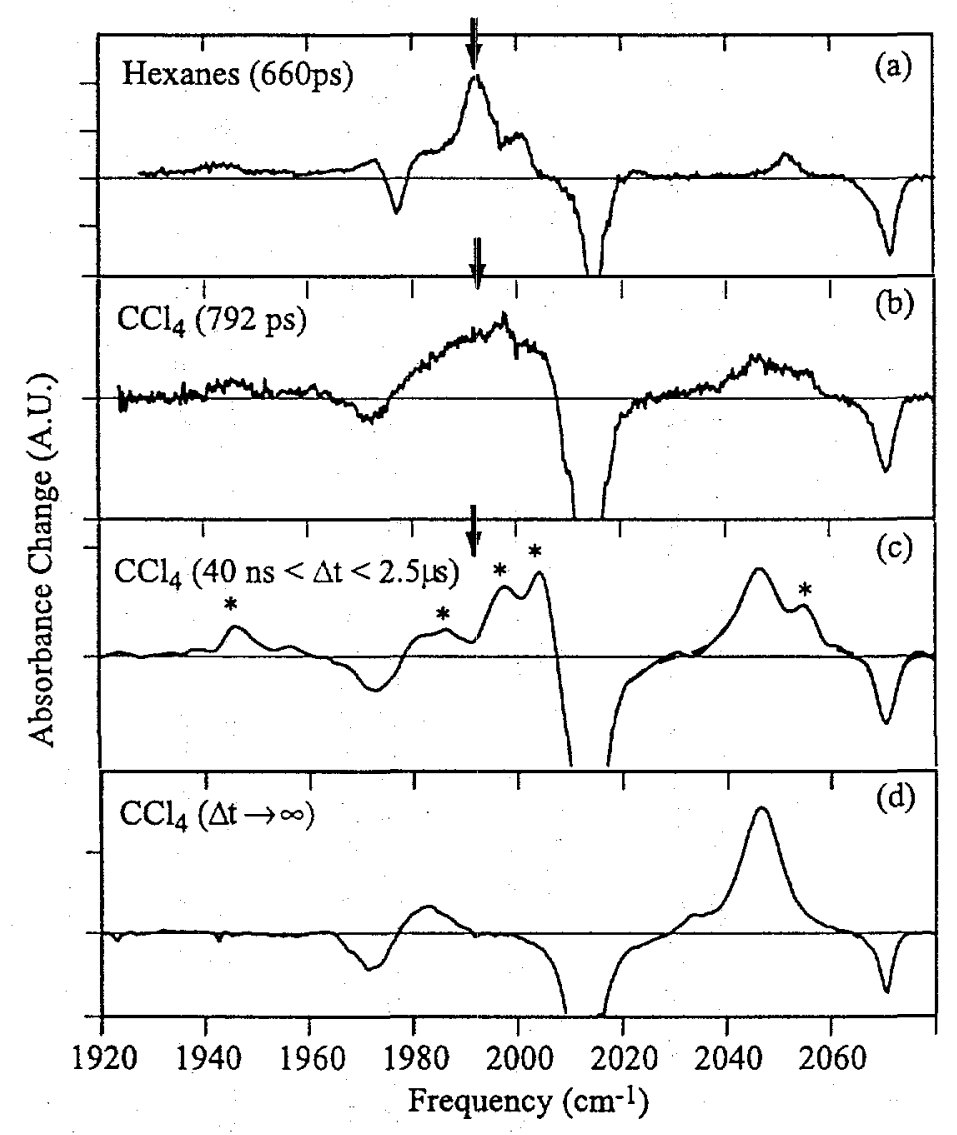

Figure 6.2 Transient difference spectra in the $\mathrm{CO}$ stretching region for $\mathrm{Re}_{2}(\mathrm{CO})_{10}$ in neat $\mathrm{CCl}_{4}$ and hexanes.

In panel (c), peaks due to $\mathrm{Re}_{2}(\mathrm{CO})_{9}\left(\mathrm{CCl}_{4}\right)$ are marked with asterisks.

$1990 \mathrm{~cm}^{-1}$ on the third panel can be attributed to the weakly solvated $\operatorname{Re}(\mathrm{CO})_{5}$ in $\mathrm{CCl}_{4}$. The similar peak positions of the $\operatorname{Re}(\mathrm{CO})_{5}$ band in $\mathrm{CCl}_{4}$ and hexanes solutions suggests that the $\operatorname{Re}(\mathrm{CO})_{5} /$ solvent interactions are of similar magnitude in these two solutions. This conclusion is supported by DFT calculations, ${ }^{1}$ which provide a qualitative estimate of the interaction energies for $\operatorname{Re}(\mathrm{CO})_{5} / \mathrm{CH}_{4}(\sim-0.2 \mathrm{kcal} / \mathrm{mol})$ and $\operatorname{Re}(\mathrm{CO})_{5} / \mathrm{CCl}_{4}(\sim-$

\footnotetext{
${ }^{1}$ The results of extensive DFT calculations for the photochemistry of $\mathrm{Re}_{2}(\mathrm{CO})_{10}$ will be described in a separate publication. The interaction energies shown here are gas-phase values. The results of extensive DFT calculations for the photochemistry of $\operatorname{Re}_{2}(\mathrm{CO})_{10}$ will be described in a separate publication. The interaction energies shown here are gas-phase values.
} 
$0.6 \mathrm{kcal} / \mathrm{mol}){ }^{1}$ Furthermore, the calculated weak interaction energy indicates that the mean thermal energy $\sim 0.6 \mathrm{kcal} / \mathrm{mol}$ at the room temperature is sufficient to disrupt the formation of a stable complex of the form $\operatorname{Re}(\mathrm{CO})_{5} \ldots$ (solvent). In other words, $a$ dynamic equilibrium is established for $\operatorname{Re}(\mathrm{CO})_{5} \ldots$ (solvent) $\leftrightarrow \operatorname{Re}(\mathrm{CO})_{5}+$ solvent [10], the time scale of which is on the order of collision in liquids ( $\sim$ few ps). This allows the chemically active $\operatorname{Re}$ center to undergo recombination reaction with another $\operatorname{Re}(\mathrm{CO})_{5}$ radical to reform the parent $\operatorname{Re}_{2}(\mathrm{CO})_{10}$ molecule. As will be shown later, the aforementioned processes in general occur on time scale orders of magnitude faster than that of the $\mathrm{C}-\mathrm{Cl}$ bond activation step, which is in the nanosecond regime.

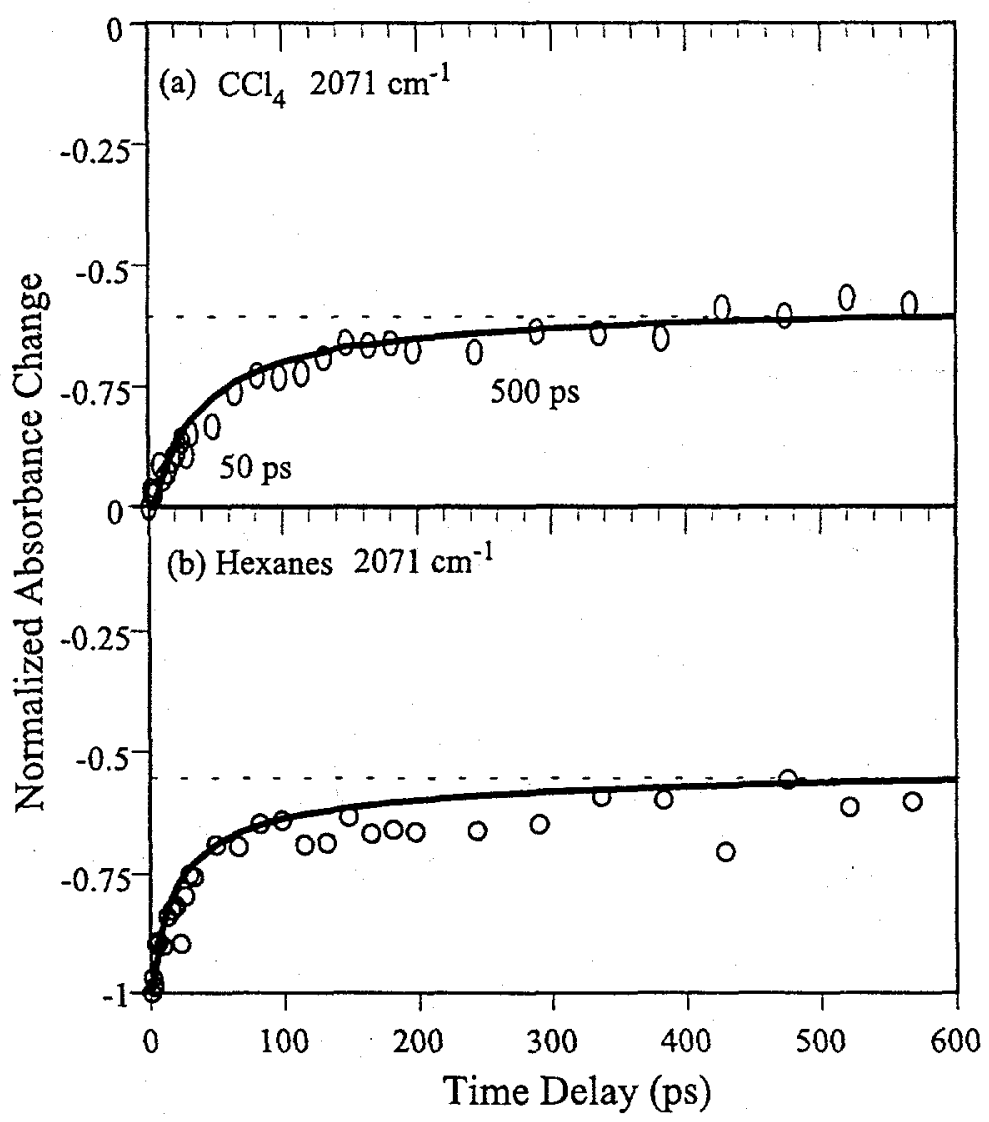

Figure 6.3 Fits of ultrafast kinetics to a diffusion model.

\footnotetext{
${ }^{1}$ The structures of these adducts are similar to those of the corresponding transition states (cf. Figure 6.4); the $\mathrm{C}-\mathrm{Cl}$...Re atoms assume a linear arrangement in the adducts.
} 
Representative parent bleach kinetics (open circles) of $\mathrm{Re}_{2}(\mathrm{CO})_{10}$ in (a) neat $\mathrm{CCl}_{4}$; and (b) hexanes solution. Fits to a diffusion model to account for geminate recombination are shown as solid lines. Except for the macroscopic viscosity, identical molecular parameters are used for all the solvents studied (hexanes, and $\mathrm{CH}_{\mathrm{n}} \mathrm{Cl}_{4-\mathrm{n}}, \mathrm{n}=0,1,2$ ).

Figure 6.3 shows the ultrafast kinetics for the parent molecule $\operatorname{Re}_{2}(\mathrm{CO})_{10}$ at 2071 $\mathrm{cm}^{-1}$ in $\mathrm{CCl}_{4}$ (Figure 6.3a) and hexanes (Figure 6.3b) solutions. The parent kinetics in $\mathrm{CCl}_{4}$ recovers on two time scales of $\sim 50$ and $\sim 500 \mathrm{ps}$ if fitted to two exponentials. Considering that the photo-initiation step is most likely a direct dissociation $(\sim 100 \mathrm{fs})$ [141] and the fact that no other transient intermediate is observed, the most likely origin for the biphasic recovery is geminate recombination of the $\operatorname{Re}(\mathrm{CO})_{5}$ pairs. To account for such dynamics, a diffusion model is adapted to reproduce the parent bleach kinetics in all the solvents studied [142-145]. According to the model, the fast 50 ps component is attributed to a convolution of vibrational relaxation and the probability of reactive collision of the $\operatorname{Re}(\mathrm{CO})_{5}$ pair within the solvent cage to form the parent $\operatorname{Re}_{2}(\mathrm{CO})_{10}$. On a longer time scale, the mean separation of the two $\operatorname{Re}(\mathrm{CO})_{5}$ fragments increases as a result of diffusive motion, thus diminishing the probability of recombination.

In the nanosecond time regime, the $\operatorname{Re}(\mathrm{CO})_{5}$ radicals that have not undergone geminate recombination ( $>50 \%$, indicated by the parent kinetics shown in Figure 3, or from the asymptotes of the diffusion model) may activate the $\mathrm{C}-\mathrm{Cl}$ bond to form the final product $(\mathrm{CO})_{5} \mathrm{ReCl}$ in chlorinated methane solutions. By monitoring the product kinetics at $2045 \mathrm{~cm}^{-1}$, the time scales for activation are found $1.33 \pm 0.06 \mathrm{~ns}$ for $\mathrm{CCl}_{4},{ }^{1}{ }^{128} \pm 40$ ns for $\mathrm{CHCl}_{3}$, and $270 \pm 60 \mathrm{~ns}$ for $\mathrm{CH}_{2} \mathrm{Cl}_{2}$. These time scales correspond to mean freeenergy barriers $\Delta \mathrm{G}^{\ddagger}$ of $5.35 \pm 0.03,8.03 \pm 0.20$, and $8.48 \pm 0.13 \mathrm{kcal} / \mathrm{mol}$, respectively. ${ }^{2}$ The fact that no other intermediates were detected prior to the product formation and that no appreciable $\mathrm{CO}$ stretch frequency shift for $\operatorname{Re}(\mathrm{CO})_{5}$ was observed suggest that the

\footnotetext{
${ }^{1}$ This time scale corresponds to a reaction rate of $(7.3 \pm 0.4) \times 10^{7} \mathrm{M}^{-1} \mathrm{~s}^{-1}$, and is in good agreement with the literature value of $(1.8-9.1) \times 10^{7} \mathrm{M}^{-1} \mathrm{~s}^{-1}$, calculated from a set of Arrhenius parameters by: Meckstroth W.K.; Reed, D.T.; Wojcicki, A. Inorg. Chim. Acta., 1985, 105, 147-151.

${ }^{2}$ The energy barriers are calculated using $1 / \tau \approx k_{T S T}=\frac{k_{B} T}{h} \exp \left(-\Delta G^{+} / R T\right), \mathrm{T}=298 \mathrm{~K}$. 
reaction involve only the rate-limiting $\mathrm{Cl}$-atom transfer step without the $19-\mathrm{e}^{-}$nor the charge-transfer intermediates.

To better understand the reactivity, the transition states for the series of reactions were studied using DFT methods. As illustrated in Figure 6.4, the results show that each transition state-obtained by locating the saddle point of the multidimensional potential energy surface of the system-can be characterized by a single imaginary frequency that involves simultaneous dissociation of the $\mathrm{C}-\mathrm{Cl}$ bond and formation of the $\mathrm{Re}-\mathrm{Cl}$ bond. The structural variation along the series of chlorinated methanes suggests that the transition state becomes more product-like as the number of hydrogen $n$ in $\mathrm{CH}_{n} \mathrm{Cl}_{4-\mathrm{n}}$ increases. For example, the $\mathrm{Re} \ldots \mathrm{Cl}$ distance in $(\mathrm{CO})_{5} \mathrm{Re} \ldots \mathrm{Cl} \ldots \mathrm{CH}_{n} \mathrm{Cl}_{3-\mathrm{n}}$ decreases monotonically from $2.95 \AA(n=0)$ to $2.84 \AA(n=1)$ to $2.76 \AA(n=2)$, and to $2.52 \AA$ for the final the product $(\mathrm{CO})_{5} \mathrm{Re}-\mathrm{Cl}$. The calculated energy barriers $\Delta \mathrm{E}_{0}^{+} \stackrel{+}{+}$ in $\mathrm{CCl}_{4}, \mathrm{CHCl}_{3}$, and $\mathrm{CH}_{2} \mathrm{Cl}_{2}$ are respectively $0.86,3.0$, and $8.5 \mathrm{kcal} / \mathrm{mol}$, where the solvent effects are treated self-consistently in the reaction-field approximation. The current level of theory, however, does not permit an immediate comparison of the experimental energy barriers to the calculated values. Nonetheless, comparison of the experimental trend and the qualitative trend from the theoretical calculations along the series of chloromethane provides greater insight in understanding the reactivity. The observed trends in the barrier and the transition-state structure are in agreement with Hammond's postulate [146], which associates a later transition state with a higher energy barrier.

\subsection{Conclusion}

In summary, femtosecond IR spectroscopy that allows for direct assessment of the roles of the transient intermediates during a reaction continues to demonstrate its ability to elucidate otherwise intricate chemical reaction mechanisms. The present work provides experimental and theoretical evidence that one-electron oxidative-addition of a 
$\mathrm{Cl}$ atom (from chlorinated methanes) to the $\operatorname{Re}(\mathrm{CO})_{5}$ radical does not involve an appreciable $19-\mathrm{e}^{-}$intermediate. Nor do the results support the idea of a charge-transfer

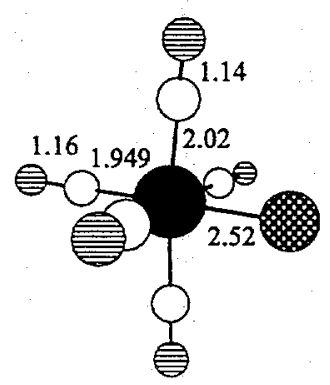

$\operatorname{Re}(\mathrm{CO})_{5} \mathrm{Cl}\left(\mathrm{C}_{4 \mathrm{~V}}\right)$

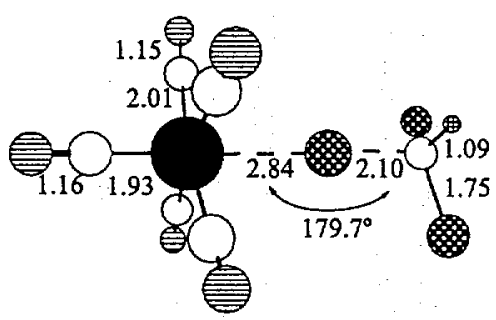

TS2 $t s-\mathrm{Re}(\mathrm{CO})_{5} \ldots \mathrm{Cl} \ldots \mathrm{CHCl}_{2}$ $\left(306 i \mathrm{~cm}^{-1}\right)$

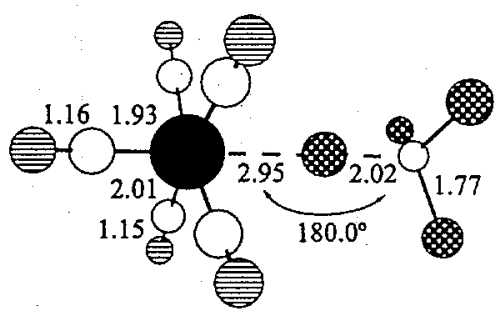

TS1 $t s-\operatorname{Re}(\mathrm{CO})_{5} \ldots \mathrm{Cl} \ldots \mathrm{CCl}_{3}$ $\left(253 i \mathrm{~cm}^{-1}\right)$

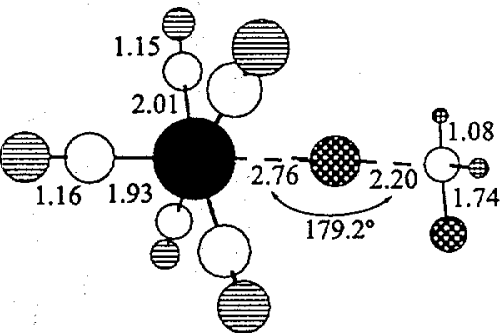

TS3 $t s-\operatorname{Re}(\mathrm{CO})_{5} \ldots \mathrm{Cl} \ldots \mathrm{CH}_{2} \mathrm{Cl}$ $\left(344 i \mathrm{~cm}^{-1}\right)$

Figure 6.4 DFT structures for the final product and transition-state structures.

Geometrical parameters are in $\AA$ for bond lengths and degree for bond angles. Also shown are the imaginary vibrational frequencies associated with each transition state.

intermediate $\left[(\mathrm{CO})_{5} \mathrm{Re}^{+}+{ }^{-} \mathrm{Cl}-\mathrm{R}\right]$ for this reaction. ${ }^{1}$ For if this is the case, the positively charged $(\mathrm{CO})_{5} \mathrm{Re}^{+}$will exhibit a substantial $v_{C O}$ shift, which was not observed. With the above evidence and the fact that no other intermediates were observed, it is suggested that the reaction be considered as involving only the $\mathrm{C}-\mathrm{Cl}$ bond activation process. Qualitatively, the observed variation in the reaction rate and DFT transition-state structure is in agreement with Hammond's postulate. More studies are needed in order to arrive at a model that is capable of predicting, even qualitatively, the reaction rates from

\footnotetext{
${ }^{1}$ There is, however, only a limited extent of charge-transfer character in the calculated transition states (Table I of the supporting material).
} 
macroscopic parameters such as the strength or electron affinity of the carbon-halogen bond $[147,148]$. 


\subsection{Supplementary}

\subsubsection{Methods}

Samples. Dirhenium decacarbonyl $\mathrm{Re}_{2}(\mathrm{CO})_{10}$ (98\%) and carbon tetrachloride $\mathrm{CCl}_{4}$ $\left(99.9 \%, \mathrm{~d}=1.589 \mathrm{~g} / \mathrm{cm}^{3}\right)$ were purchased from Aldrich, Inc.. Hexanes $\mathrm{C}_{6} \mathrm{H}_{14}(99.9 \%, \mathrm{~d}=$ $\left.0.664 \mathrm{~g} / \mathrm{cm}^{3}\right)$, trichloromethane $\mathrm{CHCl}_{3}\left(99 \%, \mathrm{~d}=1.484 \mathrm{~g} / \mathrm{cm}^{3}\right)$, and dichloromethane $\mathrm{CH}_{2} \mathrm{Cl}_{2}\left(99.9 \%, \mathrm{~d}=1.3255 \mathrm{~g} / \mathrm{cm}^{3}\right)$ were purchased from Fisher Scientific, Inc.. All chemicals were used without further purification. The sample was enclosed in an airtight, demountable liquid IR flow cell (Harrick Scientific Corporation). The concentrations of the $\operatorname{Re}_{2}(\mathrm{CO})_{10}$ solutions were approximately $7 \mathrm{mM}$ in hexanes and 4 $\mathrm{mM}$ in chlorine substituted methanes.

Femtosecond infrared spectroscopy. Details of the femtosecond IR (fs-IR) spectrometer setup have been published elsewhere [149]. In brief, the output of a Ti:sapphire oscillator was amplified in series of dye amplifiers pumped by a $30-\mathrm{Hz}$ Nd:YAG laser. The UV and IR beams used to initiate and probe the reaction were generated through nonlinear crystals. The resulting 295-nm UV photons (with energy of $\sim 6 \mu \mathrm{J} /$ pulse) were focused into a disk of $\sim 200 \mu \mathrm{m}$ diameter at the sample to initiate chemical reactions. The $\sim 1-\mu \mathrm{J}$ IR pulses having a temporal full-width-half-maximum of about $70 \mathrm{fs}$ and a spectral band width of about $200 \mathrm{~cm}^{-1}$ were split into a signal and a reference beam to minimize the shot-to-shot fluctuation and transient heating of the detector. These two beams were then focused into an astigmatism-corrected spectrographic monochromator (SpectraPro-150, Acton Research Corp., 150 grove/mm, $4.0 \mu \mathrm{m}$ blazed) to form two spectrally resolved images on a focal-plane-array (FPA) detector. The two frequency-resolved images were digitized by two windows of $12 \times 200$ pixels, which allowed simultaneous normalization of a $\sim 70 \mathrm{~cm}^{-1}$ spectrum. The censoring chip of the detector was an engineer grade, 256×256-element $\mathrm{HgCdTe}(\mathrm{MCT})$ 
matrix of dimensions $1.28 \times 1.28 \mathrm{~cm}^{2}$ (or $50 \times 50 \mu \mathrm{m}^{2}$ per pixel). During the course of an experiment, the censoring chip and its immediate circuits were kept in contact with a 4-L liquid nitrogen dewar to increase its sensitivity in the IR range. The long-time temperature drift was minimized by normalizing the gain against the FPA readouts from a small region far away from the illuminated area. Rejection of bad laser shots further improved the $\mathrm{S} / \mathrm{N}$ ratio. With a data acceptance ratio of about 0.5 and a $30-\mathrm{Hz}$ laser repetition rate, it took about 1 minute to acquire signals on the order of $1 \%$ absorbance change with 10:1 S/N ratio after signal averaging for 1000 valid laser shots. The typical spectral and temporal resolution for this setup were $\sim 4 \mathrm{~cm}^{-1}$ and $\sim 300 \mathrm{fs}$, respectively. The polarizations of the pump and the probe pulse were set at the magic angle $\left(54.7^{\circ}\right)$ to ensure that all signals were due to population dynamics. All kinetic data have been corrected for a positive chirp of $\sim 1.4 \mathrm{fs} / \mathrm{cm}^{-1}$, measured from pump-probe cross correlation using a silicon wafer. A broad, wavelength independent background signal from $\mathrm{CaF}_{2}$ windows has also been subtracted from the transient spectra and kinetic traces.

Nanosecond step-scan FTIR. Measurements were made using a Step-Scan FTIR spectrometer described elsewhere [150]. The instrument was based on a Bruker IFS-88 FTIR with a special scanner module to allow step-scanning. An InSb detector with a 40ns temporal full-width-half-maximum (FWHM) measured from the IR scatter of 1064$\mathrm{nm}$ light from a YAG laser was used. The IR light was focused in the cavity with two 10-mm focal length $\mathrm{BaF}_{2}$ lenses, which gave beam sizes smaller than comparable curved mirrors, allowing increased IR throughput and less sample degradation. The sample was photo-excited with 10-ns pulses from the second harmonic of a dye laser tuned to 590 $\mathrm{nm}$.

Theoretical. In this communication, the gas-phase interaction energies for the metal fragment / solvent molecule pairs $\operatorname{Re}(\mathrm{CO})_{5} / \mathrm{CH}_{4}$ and $\operatorname{Re}(\mathrm{CO})_{5} / \mathrm{CCl}_{4}$ are reported. For the $\mathrm{Cl}$-atom abstraction reaction, we compute the system $(\mathrm{CO})_{5} \mathrm{Re} \ldots \mathrm{CH}_{\mathrm{n}} \mathrm{Cl}_{4-\mathrm{n}}(\mathrm{n}=0,1$, 2) at three critical stages along the reaction coordinate: the precursor complex, the 
transition state, and the final product. In computing the reactive system, the solvent effects are treated self-consistently in the reaction-field approximation. The exchangecorrelation functional employed in our density functional theoretical (DFT) calculations was Becke's three-parameter hybrid functional [151] combined with the Lee-Yang-Parr correlation functional [152], commonly denoted as B3LYP [153]. This functional has been shown to give very good results for transition metal complexes $[154,155]$. Except for complexes with apparent symmetry, no constraint was imposed during geometry optimization. To make certain that a proper energy extremum-obtained by locating the local extremum that has a positive second energy derivative with respect to geometry change-had been located each geometry optimization was followed by a frequency calculation at the same level of theory. Two basis sets were used in the calculations. Basis set $\mathrm{I}$ consisted of the $6-31 \mathrm{G}$ basis functions for $\mathrm{H}, \mathrm{C}, \mathrm{O}$, and $\mathrm{Cl}$ atoms $[156,157]$, and the Los Alamos Effective Core Potential (ECP) for Re with the outer-most core orbitals included in the valence description $(\mathrm{ECP}+5 \mathrm{~s} 5 \mathrm{p} 6 \mathrm{~s} 5 \mathrm{~d} 6 \mathrm{p})[158]$. Basis set II augmented basis set I by placing polarization functions on all atoms except Re. The smaller basis set I was used for geometry optimization of the ax- and eq$\mathrm{Re}_{2}(\mathrm{CO})_{9}$ (solvent) complexes. All other complexes were optimized using basis set II. It is well known that it is difficult to obtain a consistent and accurate estimate of the basis set superposition error (BSSE) along the course of a chemical reaction [159]. Similar difficulty was also experienced in the course of this work. Therefore, in order to describe the potential energy surface in a consistent way, the binding energy $\Delta \mathrm{E}$ for a complex $\mathrm{XY}$ was referenced against the energy of an optimized supramolecular configuration where the $\mathrm{X}$ and $\mathrm{Y}$ fragments were held at $10 \AA$ apart. The $\Delta \mathrm{E}_{0}^{\ddagger}$ reported in the article were corrected for the zero-point energy at $298 \mathrm{~K}$. The commercial JAGUAR package was used for the DFT calculations [160].

\subsubsection{Diffusion Model for Geminate Recombination of $\operatorname{Re}(\mathrm{CO})_{5}$ Radicals.}


The microscopic picture for the competing processes of geminate recombination and atom abstraction-including non-equilibrium barrier crossing, vibration relaxation, solvent caging, $\mathrm{Cl}$-atom abstraction rate $\left(k_{a}\right)$ and geminate recombination rate $\left(k_{\text {rec }}\right)$-can be obtained by incorporating appropriate theoretical models when analyzing the data. In the current work, the data are analyzed following the treatment by Naqvi and others [142]. Because of the transparent physical picture, models of the same spirit have been successfully applied to analysis of various reaction pair dynamics [143, 161-163]. For completeness, the model is briefly outlined below.

\section{Equation 6.1}

$$
(\mathrm{CO})_{5} \mathrm{MM}^{\prime}(\mathrm{CO})_{5} \underset{(i i i)}{\stackrel{h \nu}{\rightleftharpoons}} \begin{cases}\mathrm{MM}^{\prime}(\mathrm{CO})_{9}+\mathrm{CO} & \text { (i) } \\ \mathrm{M}(\mathrm{CO})_{5}+\mathrm{M}^{\prime}(\mathrm{CO})_{5} & \text { (ii) }\end{cases}
$$

Consider a pair of $\operatorname{Re}(\mathrm{CO})_{5}$ radicals rest at $r_{0}$ apart at time $t \rightarrow 0$. The two radicals can react to form $\operatorname{Re}_{2}(\mathrm{CO})_{10}$ when their separation $r$ is equal to or smaller than a critical distance $R$. The time-dependent survival probability density $w(t, r ; r o, R)$ of a pair of monomers $\operatorname{Re}(\mathrm{CO})_{5}$ is considered to be governed by the diffusion equation,

Equation 6.2 $\frac{\partial}{\partial t} w\left(r, t ; r_{0}, R\right)=D \nabla^{2} w\left(r, t ; r_{0}, R\right)$,

subject to the initial condition,

\section{Equation 6.3}

$$
w\left(r, t \rightarrow 0 ; r_{0}, R\right)=\frac{1}{4 \pi r^{2}} \delta\left(r-r_{0}\right),
$$

and the boundary condition,

Equation 6.4 $\quad \lim _{r \rightarrow \infty} w\left(r, t ; r_{0}, R\right) \rightarrow 0$.

In the above equations, $D$ is the diffusion coefficient of the monomer in the solvent, $r_{0}$ the initial separation of the geminate pair at $t \rightarrow 0$, and $R$ the contact distance where the recombination reaction occurs. The diffusion coefficient $D$ is expressed by StokesEinstein equation subject to non-slip boundary condition, 


\section{Equation 6.5 $D=k_{B} T / 6 \pi \eta a$,}

where $\eta$ is the macroscopic viscosity of the solvent, and $a$ the hydrodynamic radius of the solute. In this study, we assume $a=R$. To account for the finite recombination rate, an additional boundary condition at $r=R$ is imposed,

\section{Equation 6.6}

$$
D \frac{\partial}{\partial t} w\left(r, t ; r_{0}, R\right)_{r=R}=b w\left(r, t ; r_{0}, R\right)
$$

Known as the "radiation boundary condition," Equation 6.6 states that the recombination rate is proportional to the concentration gradient at the contact distance $R$. The solution to Equation 6.2 has been explicitly worked out and is given by [164],

\section{Equation 6.7}

$$
\begin{aligned}
w\left(r, t ; r_{0}, R\right) & =\frac{1}{8 \pi r_{0} R \sqrt{\pi D t}}\left\{\exp \left[-\left(r-r_{0}\right)^{2} / 4 D t\right]+\exp \left[-\left(r+r_{0}-2 R\right)^{2} / 4 D t\right]\right. \\
& \left.-2 B \sqrt{\pi D t} \exp \left[B^{2} D t+B\left(r+r_{0}-2 R\right)\right] \operatorname{erfc}\left[B \sqrt{D t}+\left(r-r_{0}\right) / 2 \sqrt{D t}\right]\right\}
\end{aligned}
$$

where $B \equiv b / D+1 / R$ and erfc is the complementary error function given by $\operatorname{erfc}(x)=\frac{2}{\sqrt{\pi}} \int_{x}^{\infty} d u \exp \left(-u^{2}\right)$. The relation of the proportionality $b$ in Equation 6.6 to the recombination rate constant can be expressed by,

Equation 6.8 $\quad k_{r e c}=4 \pi R^{2} b$.

Thus, the time-dependent concentration of the monomer $\operatorname{Re}(\mathrm{CO})_{5}$ is given by,

Equation $6.9 \quad M\left(t ; r_{0}, R\right)=\int_{R}^{\infty} d r 4 \pi r^{2} w\left(r, t ; r_{0}, R\right)$

Similarly, the instantaneous probability for formation of the parent molecule is,

Equation 6.10 $\quad p\left(t ; r_{0}, R\right)=4 \pi R^{2} b w\left(r=R, t ; r_{0}, R\right)$.

When the model is applied to analysis of the parent bleach recovery, the accumulating parent molecules are accounted for by integration of Equation 6.10 over time, 
Equation 6.11

$$
P\left(t ; r_{0}, R\right)=\int_{0}^{t} d t^{\prime} p\left(t^{\prime} ; r_{0}, R\right)
$$

In the above model, the initial condition implies that the time scale of dissociation is much shorter than that of subsequent geminate-recombination. This assumption is justified in view of the similarity in the electronic structure of $\mathrm{M}_{2}(\mathrm{CO})_{10}(\mathrm{M}=\mathrm{Mn}, \mathrm{Re})$ and a recent experiment on the photodissociation of $\mathrm{Mn}_{2}(\mathrm{CO})_{10}$, which shows that the time scale of $\mathrm{Mn}-\mathrm{Mn}$ bond cleavage is on the order of $100 \mathrm{fs}$ [141]. To apply the model to Equation 6.1, let $Q_{i}$ and $Q_{i i}$ denote the fraction of parent molecules that dissociate via pathways (i) and (ii), respectively. It is assumed that all the excited parent molecules dissociate following either pathway (i) or (ii) only; in other words, $Q_{i}+Q_{i i}=1$. Due to solvation of $\operatorname{Re}_{2}(\mathrm{CO})_{9}$ in pathway (i), geminate recombination of $\mathrm{CO}$ and $\operatorname{Re}_{2}(\mathrm{CO})_{9}$ dose not contribute appreciably to the reformation of the parent molecule. Photofragments generated via pathway (ii) are therefore the main source for geminate recombination. The model is further simplified by assuming that a single rate constant $k_{r e c}$ describes vibrational cooling, non-dissociative energy relaxation, and primary geminate recombination. In this way, the normalized parent bleach signal can be written as,

Equation 6.12

$$
I_{\text {parent }}(t)=Q_{i i}\left[-1+P\left(t ; r_{0}, R\right)\right]-Q_{i}=-1+Q_{i i} \times P\left(t ; r_{0}, R\right)
$$

The physical parameters needed for the analysis of the experimental data are: the branching ratio $Q_{i i}$, the solvent viscosity $\eta$, the contact distance $R$, the initial separation $r_{0}$, and the recombination rate constant $k_{\text {rec }}$. The contact distance $R$ is assigned as twice of the distance from the center of $\mathrm{Re}-\mathrm{Re}$ bond to the center of the axial $\mathrm{Re}-\mathrm{C}-\mathrm{O}$ line. Similar treatments have been employed in the analysis of geminate pair dynamics of disulfides $[143,144]$. Using the crystallographic data of $\operatorname{Re}_{2}(C O)_{10}$ [145], $R$ is estimated to be $6.256 \AA$. To verify this estimate, the DFT geometry for the $\operatorname{Re}(\mathrm{CO})_{5}$ radical, and the Lennard-Jones parameters for the $\mathrm{C}$ and $\mathrm{O}$ atoms are used to construct a LennardJones $\operatorname{Re}(\mathrm{CO})_{5}$ molecule suitable for classical molecular simulation [165]. The contact 
distance in this approach is defined as the diameter of a sphere that encloses the LennardJones $\operatorname{Re}(\mathrm{CO})_{5}$. This procedure gives a contact distance of $6.209 \AA$, consistent with the first estimate.

For the reaction in $\mathrm{CCl}_{4}$ solution $\left(\eta_{20}{ }^{\circ} \mathrm{C}=0.97 \mathrm{cp}\right)$, the product kinetics and parent bleach signal of are fitted simultaneously since they draw from the same $\operatorname{Re}(\mathrm{CO})_{5}$ source. Three processes are considered to contribute to the observed product kinetics: vibrational cooling, non-equilibrium barrier crossing to form the product, and equilibrium barrier crossing. Incorporating these ideas, the normalized product signal is described as,

\section{Equation 6.13}

$$
I_{\text {product }}(t)=A_{v i b} \exp \left(-t / \tau_{v i b}\right)+A_{\text {neq }}\left(1-\exp \left(-t / \tau_{\text {neq }}\right)+\int_{0}^{t} d t^{\prime} k_{a} M\left(t^{\prime} ; r_{0}, R\right)\right.
$$

The first term of Equation 6.13 describes the relaxation of overlapped low-frequency modes; the second term models the non-equilibrium product formation with a phenomenological rise time $\tau_{\text {neq }}$; and the last term relates the time-dependent $\operatorname{Re}(\mathrm{CO})_{5}$ concentration $M\left(t^{\prime} ; r_{0}, R\right)$ from Equation 6.9 to the macroscopic, pseudo first-order rate constant $k_{a}$. To account for the non-equilibrium barrier crossing in the $\mathrm{CCl}_{4}$ case, the normalized parent signal in Equation 6.12 becomes,

\section{Equation 6.14}

$$
I_{\text {parent }}(t)=-1+\left(1-A_{n e q}\right) \times Q_{i i} \times P\left(t ; r_{0}, R\right) \text {. }
$$

With $R$ fixed $(=6.256 \AA)$ and $Q_{i i}(=0.8)$ obtained from experiments, the recombination rate is found to be $k_{r e c}=(1.0 \pm 0.2) \times 10^{11} \mathrm{M}^{-1} \mathrm{~s}^{-1}$, greater than the literature value measured in $\mathrm{CH}_{3} \mathrm{CN},(1.0 \pm 0.2) \times 10^{10} \mathrm{M}^{-1} \mathrm{~s}^{-1}$ [166]. This is not surprising since in the current simplified model, a single parameter $k_{r e c}$ is used to encompass all fast dynamics such as non-dissociative energy relaxation and primary geminate-recombination. The initial separation of the two $\operatorname{Re}(\mathrm{CO})_{5}$ monomers was found to be $r_{0}=8.6 \pm 0.2 \AA$, about $2.3 \AA$ away from their contact distance. The Cl-atom abstraction rate $k_{a}=(7.5 \pm 0.4) \times 10^{8} \mathrm{~s}^{-1}$ $\left((7.3 \pm 0.4) \times 10^{7} \mathrm{M}^{-1} \mathrm{~s}^{-1}\right)$ is in very good agreement with the literature value, which is calculated to be $(1.8-9.1) \times 10^{7} \quad \mathrm{M}^{-1} \mathrm{~s}^{-1}$ from a set of Arrhenius parameters: 
$k=A \exp \left(-\frac{E_{a}}{R T}\right)$, with $A=(2.8 \pm 0.5) \times 10^{9} \mathrm{M}^{-1} \mathrm{~s}^{-1}$ and $E_{a}=2.5 \pm 0.4 \mathrm{kcal} / \mathrm{mol}$ in ethanol [167]. The time constant for the fast rise of product kinetics $\tau_{\text {neq }}$ is found to be $2.3 \pm 0.9$ ps, with an amplitude $A_{n e q}=0.10 \pm 0.01$. That is, about $10 \%$ of the product was produced through non-equilibrium barrier crossing. Finally, the hot band intensity $A_{v i b}$ is found to be $0.13 \pm 0.01$ with a vibrational-cooling time $\tau_{v i b}=35 \pm 14 \mathrm{ps}$. The resulting traces for the product and parent kinetics are plotted on top of the experimental data displayed in Figure 6.5.

Application of the above model to the other solvents, $\mathrm{CHCl}_{3}\left(\eta_{20}{ }^{\circ} \mathrm{C}=0.58 \mathrm{cp}\right)$, $\mathrm{CH}_{2} \mathrm{Cl}_{2}\left(\eta_{20}{ }^{\circ} \mathrm{C}=0.45 \mathrm{cp}\right)$, and hexane $\left(\eta_{20}{ }^{\circ} \mathrm{C}=0.33 \mathrm{cp}\right)$ demonstrates that the model captures the essential physics underlying the dynamics of the $\operatorname{Re}(\mathrm{CO})_{5}$ monomers. Note that except for the bulk viscosity, all the parameters used in these plots are identical to those obtained from modeling of $\operatorname{Re}_{2}(\mathrm{CO})_{10} / \mathrm{CCl}_{4}$.

Table 6.1. Summary of Electron-Population Analysis for the $\mathrm{Re}$ and $\mathrm{Cl}$ Centers Involved in the Reaction of Cl-Atom Abstraction by the $\operatorname{Re}(\mathrm{CO})_{5}$ Radical.

\begin{tabular}{lllll}
\hline $\begin{array}{l}\text { Complexes } \\
\text { Atomic Center }\end{array}$ & $\begin{array}{l}\text { Mulliken Charge } \\
\mathrm{Re}\end{array}$ & $\mathrm{Cl}^{\mathrm{a}}$ & $\begin{array}{l}\text { Natural Charge } \\
\mathrm{Re}\end{array}$ & $\mathrm{Cl}^{\mathrm{b}}$ \\
\hline Transition State & & & & \\
$t s-(\mathrm{CO})_{5} \mathrm{Re} . . . \mathrm{Cl} \ldots \mathrm{CCl}_{3}$ & -0.222 & -0.025 & -0.481 & -0.168 \\
$t s-(\mathrm{CO})_{5} \mathrm{Re} . . \mathrm{Cl} \ldots \mathrm{CHCl}_{2}$ & -0.244 & -0.0925 & -0.496 & -0.260 \\
$t s-(\mathrm{CO})_{5} \mathrm{Re} \ldots \mathrm{Cl} \ldots \mathrm{CH}_{2} \mathrm{Cl}$ & -0.264 & -0.144 & -0.508 & -0.331 \\
Product & & & & \\
$(\mathrm{CO})_{5} \mathrm{ReCl}$ & -0.321 & -0.209 & -0.567 & -0.477 \\
\hline
\end{tabular}

a Mulliken charge population for a $\mathrm{Cl}$ atom in $\mathrm{CCl}_{4}$ is $+0.090 \mathrm{e}^{-}$.

'Natural charge population for a $\mathrm{Cl}$ atom in $\mathrm{CCl}_{4}$ is $+0.068 \mathrm{e}^{-}$. 


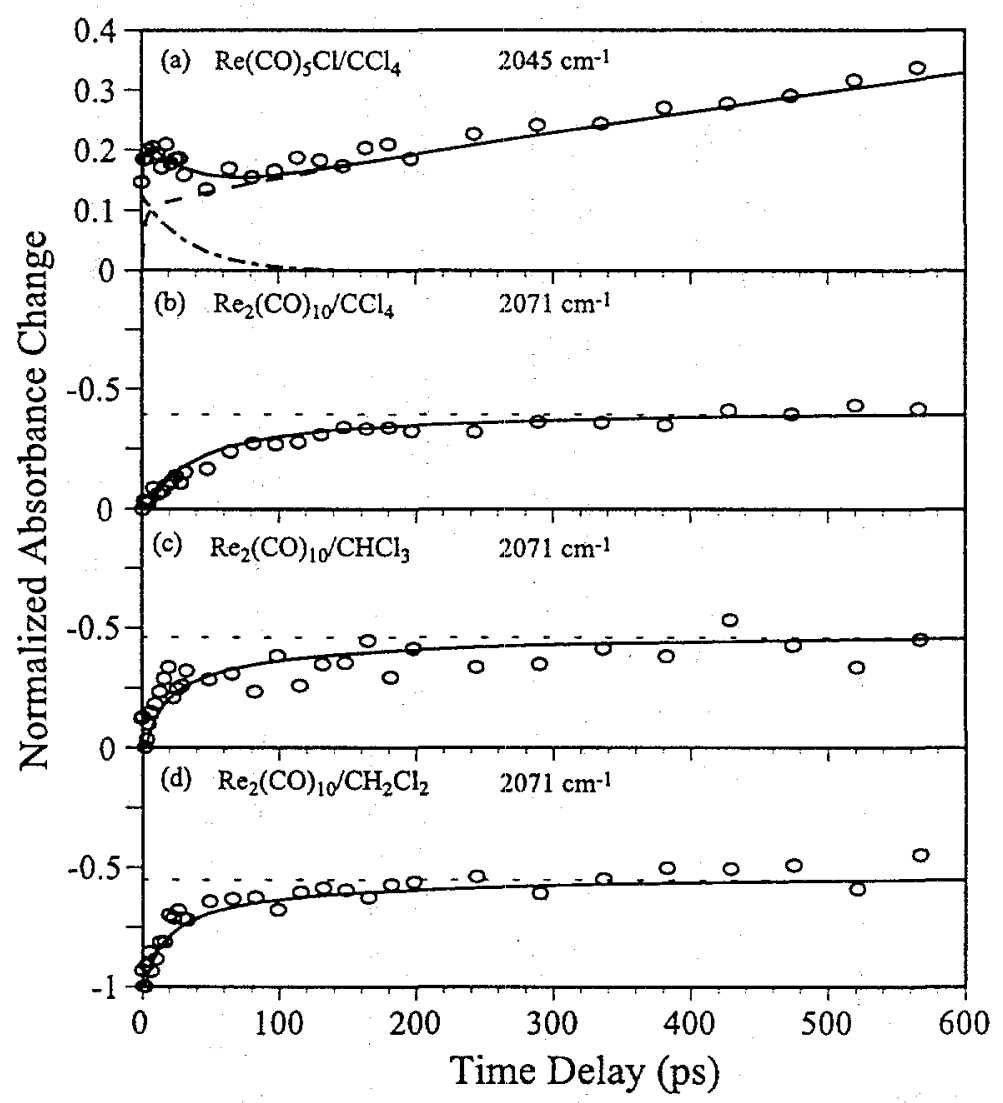

Figure 6.5 Detailed fitting of ultrafast kinetics to a diffusion model.

Overlays of the diffusion model (solid lines) for geminate-recombination dynamics and experimental data (open circles). (a) The kinetic trace of the product $\operatorname{Re}(\mathrm{CO})_{5} \mathrm{Cl}(2045$ $\mathrm{cm}^{-1}$ ) in neat $\mathrm{CCl}_{4}$ solution. The dashed line is the fit to the rise of the product band and the dash-dot line describes the decay of vibrationally excited $\mathrm{CO}$ bands. The parent $\left(\mathrm{Re}_{2}(\mathrm{CO})_{10}, 2071 \mathrm{~cm}^{-1}\right)$ kinetic traces in $\mathrm{CCl}_{4}, \mathrm{CHCl}_{3}$, and $\mathrm{CH}_{2} \mathrm{Cl}_{2}$ are displayed in (b), (c), and (d). The dotted lines indicate the asymptotic values of the diffusion model. 


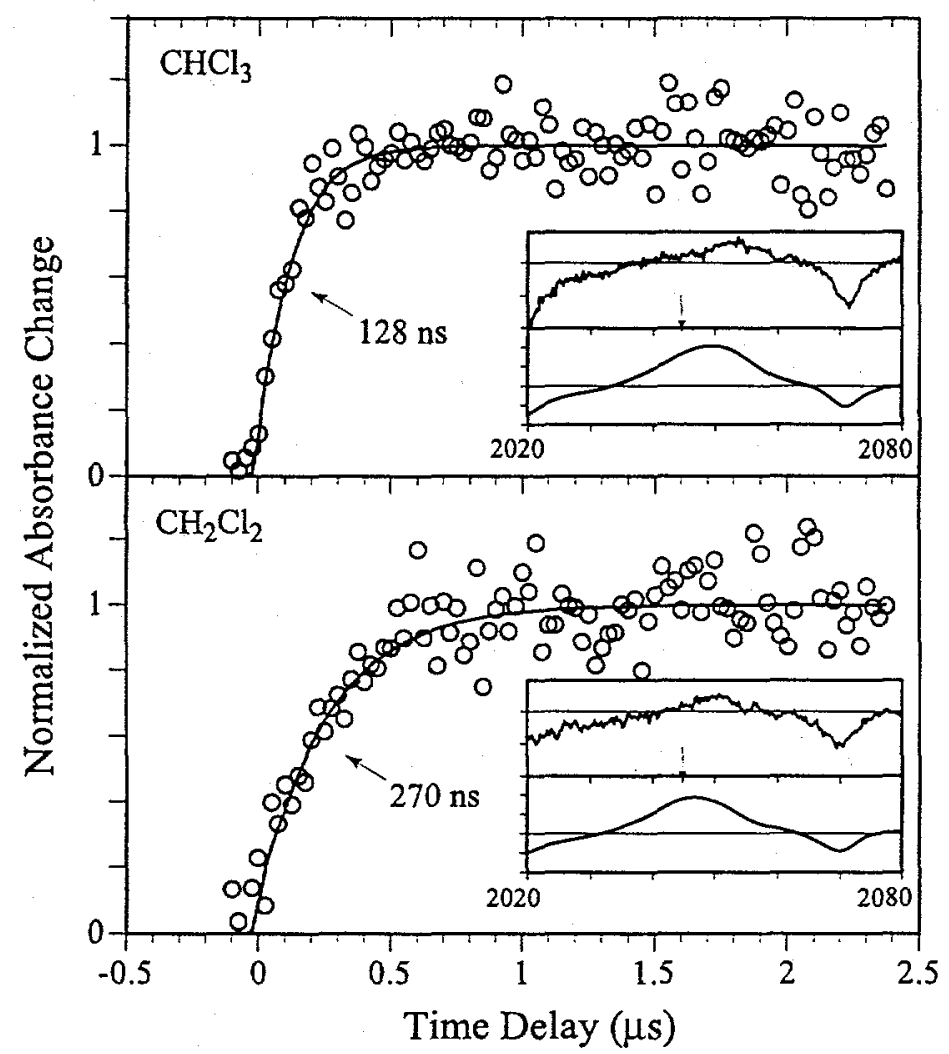

Figure 6.6 Nanosecond kinetics of $\mathrm{Cl}$-atom abstraction reaction.

Nanosecond kinetics for $\mathrm{Re}(\mathrm{CO})_{5} \mathrm{Cl}$ in $\mathrm{CHCl}_{3}$ and $\mathrm{CH}_{2} \mathrm{Cl}_{2}$ taken at the $2045 \mathrm{~cm}^{-1}$. Inserts: The top panel is an ultrafast IR spectrum recorded at 660-ps delay showing a single band due to eq- $\operatorname{Re}_{2}(\mathrm{CO})_{9}$ (solvent); the bottom panel is a nanosecond spectrum averaged over 1-2.5 $\mu \mathrm{s}$. The down-pointing arrow indicates where the product band should be. On each nanosecond spectrum, the nonacarbonyl band $\left(\sim 2055 \mathrm{~cm}^{-1}\right)$ overlaps with the product band $\left(-2045 \mathrm{~cm}^{-1}\right)$ in this spectral region, such that the two bands coalesce into one broader band. 


\section{ConClusion}

Our understanding for the reactivity of complex chemical systems such as organometallic compounds has greatly advanced in the past decade owing to the vast development of experimental and computational techniques. Basic principles born of these collective efforts have guided the thinking of reactions in fields including chemistry, biological sciences, and material sciences. Yet, the critical examination of the postulates in the realistic solution phase has been a daunting challenge due to the intricate liquid dynamics, which render the relevant time scales to spreading several orders of magnitude. Femtosecond infrared spectroscopy, which is capable of "real-time" observation and characterization of a chemical reaction, has been utilized to follow oxidative-addition reactions of prototypical two-electron $\mathrm{C}-\mathrm{H}$ and $\mathrm{Si}-\mathrm{H}$ bonds, and oneelectron $\mathrm{Cl}$ atom to organometallic complexes. This technique affords the description of not only the static reaction mechanism but also the dynamics of each elementary step, including vibrational cooling, geminate-pair recombination, morphologic reorganization, solvation-assisted intersystem crossing, and solvent rearrangement.

The approach described in this work may offer the prospect of providing new perspectives for reactions, the relevant dynamics of which take place on the ultrafast time scale. For example, a photochemical reaction such as those discussed in Chapters 4 and 5 can easily span several orders of magnitude in time from its initiation to completion, as results of the intricate dynamic processes in solution. Following photo-excitation, the reaction may begin with a dynamic partitioning in the dissociative excited state that, on the $\sim 100 \mathrm{fs}$ timescale, leads to reactive intermediates such as $\eta^{5}-\mathrm{CpMn}(\mathrm{CO})_{2}$ in either the singlet or triplet spin states. The nascent singlet $\eta^{5}-\mathrm{CpM}(\mathrm{CO})_{2}$ may interact either with the chemically inert ethyl site of $\mathrm{Et}_{3} \mathrm{SiH}$ to form the ethyl solvated $\eta^{5}-\mathrm{CpM}(\mathrm{CO})_{2}\left(\mathrm{Et}_{3} \mathrm{SiH}\right)$, or with the reactive $\mathrm{Si}-\mathrm{H}$ bond to form the final product. Under 
the dynamic influence of the solvent bath, the weakly coupled ethyl-solvate together with the surrounding solvent shell may undergo various reorganization until the metal center encounters a reactive $\mathrm{Si}-\mathrm{H}$ bond to complete the reaction. The timescale of such a procession, ranging from hundreds of picoseconds to a few microseconds, is expected to depend upon the specific metal-alkane interactions, the number of active sites in a solvent molecule, and steric interactions. Therefore, the macroscopic reaction rate is determined by the rearrangement process, which sets the time scales for the two solvation-partitioned product-formation pathways 3 decades apart.

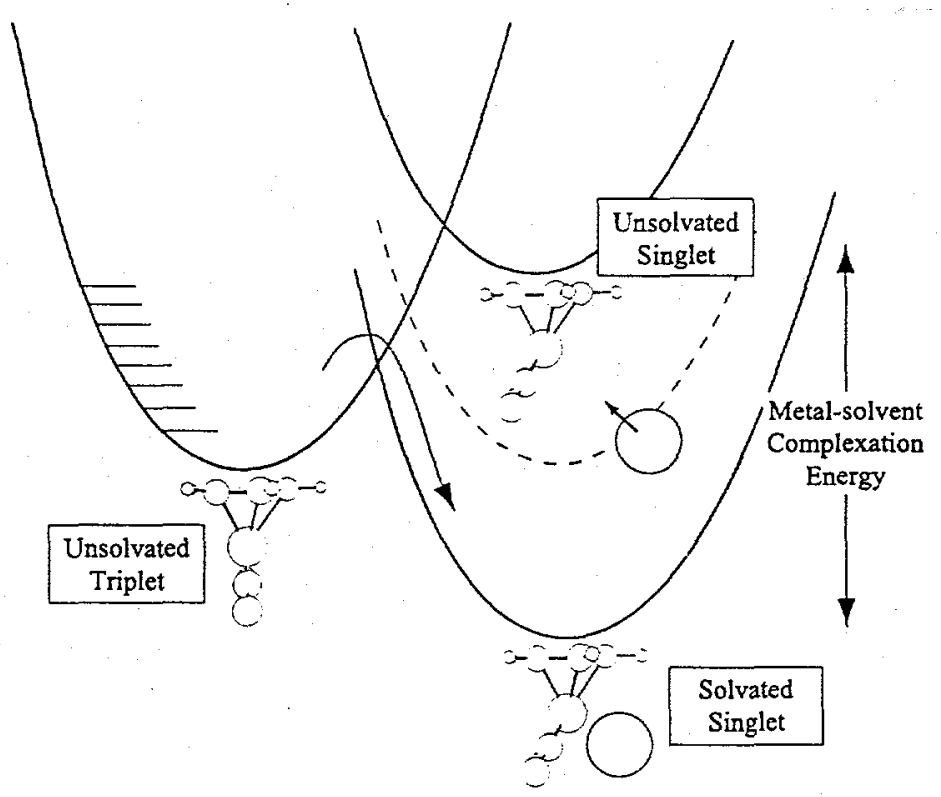

Figure 7.1 An illustration for solvation / spin crossover process.

Another important aspect brought to light by the aforementioned study is the realization of the dynamics of a high-spin, 16-electron transition-metal center in twoelectron oxidative-addition reaction. As noted earlier, transition metal mediated reactions normally occur at an unsaturated metal center that may potentially exist in more than one spin state. Conventional thinking advises that if such a reaction begins with an $S=0$ metal center, for example, the system will follow a reaction coordinate in the same electron-spin manifold. This thinking, together with the prevailing postulate that most 
organometallic reactions can be understood by invoking 16- or 18-electron intermediates or transition states [168], has been influential in describing a reaction mechanism. It is not until recently that the importance of spin-state changes in the reactivity of unsaturated transition metal complexes has been recognized $[169,170]$. For instance, a recent study: by Bengali, Bergman, and Moore shows that photogenerated $\eta^{5}-\mathrm{CpCo}(\mathrm{CO})$ or $\eta^{5}-\mathrm{Cp} * \mathrm{Co}(\mathrm{CO})$ do not form stable adducts with alkanes or rare-gas atoms $(\mathrm{Xe}, \mathrm{Kr})$ but react readily with $\mathrm{CO}$ molecules at a diffusion-limited rate [105]. Utilizing both DFT and $a b$ initio computational methods, Siegbahn later attributed these observations to an $\eta^{5}-\mathrm{CpCo}(\mathrm{CO})$ species in the ground triplet manifold. The triplet $\eta^{5}-\mathrm{CpCo}(\mathrm{CO})$ then undergoes a rapid spin-flip to form the singlet $\eta^{5}-\mathrm{CpCo}(\mathrm{CO})_{2}$ under the influence of an incoming $\mathrm{CO}$, but not when the incoming ligand is a weaker-binding alkane molecule $[106,171,172]$. Ultrafast infrared studies by Dougherty and Heilweil show that $\eta^{5}-\mathrm{CpCo}(\mathrm{CO})$ reacts very quickly (faster than vibrational cooling time) with the stronglybinding ligand 1-hexene to form presumably a singlet $\pi$-complex, in which a 1 -hexene molecule complexes to the $\mathrm{Co}$ metal through its $\mathrm{C}=\mathrm{C}$ double bond [120]. A similar reactivity has also been observed in the reaction of $\mathrm{CO}$ and $\mathrm{N}_{2}$ with triplet $\eta^{3}-\mathrm{Tp}^{i-\mathrm{Pr}, \mathrm{Me}} \mathrm{Co}(\mathrm{CO})\left(\mathrm{Tp}^{i-\mathrm{Pr}_{\mathrm{r}} \mathrm{Me}}=\mathrm{HB}-\mathrm{Pz}_{3}^{i-\mathrm{Pr}_{\mathrm{r}} \mathrm{Me}}, \mathrm{Pz}^{i-\mathrm{Pr}_{\mathrm{r}} \mathrm{Me}}=3\right.$-iso-propyl-5-methylpyrazolyl $)$ [130] or $\eta^{5}-\mathrm{Cp} * \mathrm{MoCl}\left(\mathrm{PMe}_{3}\right)[173,174]$, in the oxidative-addition of benzene or aldehyde $\mathrm{C}-\mathrm{H}$ bonds to unsaturated $\eta^{5}-\mathrm{Cp} * \mathrm{Co}\left(\eta^{2}-\mathrm{H}_{2} \mathrm{C}=\mathrm{CHSiMe}_{3}\right)$ [175-177], and most recently in the silane $\mathrm{Si}-\mathrm{H}$ bond activation by $\eta^{5}-\mathrm{CpV}(\mathrm{CO})_{4}$ [178]. In view of the above examples. it would seem that a stronger metal-ligand interaction tends to facilitate a high-spin to low-spin crossover in an organometallic compound. Although such an internolecular process can be qualitatively described by Figure 7.1, substantial efforts will be required, both in experiments and in theoretical development, to reach the same level of understanding as intramolecular intersystem crossing [179]. The unique information provided by ultrafast infrared spectroscopy, which includes the dynamics of IVR and those of molecular morphology-change, is expected to be crucial in the future developments. 
Finally, the explicit reaction scheme composed of elementary steps allows an experimental assessment for the current understanding of the reactivity. For $\mathrm{C}-\mathrm{H}$ and $\mathrm{Si}-$ $\mathrm{H}$ bond activation, the present results corroborate the prevailing picture of the reactivity. For $\mathrm{Cl}$-atom abstraction by the $\mathrm{Re}(\mathrm{CO})_{5}$ radical, however, the present results call for reexamination of the notion of a 19-electron species or a charge-transfer complex as reactive intermediates. In addition, in each of the reactions discussed the ratedetermining process has been determined to be the bond-breaking step for $\mathrm{C}-\mathrm{H}$ activation, solvent rearrangement for Si-H activation [180], and atom-transfer step for $\mathrm{C}-$ $\mathrm{Cl}$ activation. They represent three stereotypical rate-limiting steps that are expected to be common to solution-phase reactions. More examples are anticipated to emerge as more efforts are devoted to the study of complicated chemical processes, which, in time, will better our picture of reactions in liquids. 


\section{APPENDICES}

\subsection{Example Input Files for the Quantum Chemical Calculation Package GAMESS}

\subsubsection{The Input File for Calculating the CASSCF-PT2 Energy of the Singlet $\operatorname{CpMn}(\mathrm{CO})_{2}$.}

The input file included in this section demonstrates the use of GAMESS to calculate the CASSCF energy to the second order of many-particle perturbation. One firstly calculates the ROHF wavefunction with the necessary localization directives as documented in the calculation logs. The localization of the Hartree-Fock orbitals is necessary for the CASSCF wavefunctions to converge successfully. The ordering of the localized HF wavefunctions may not necessarily be in the same order as how the activate space is constructed. As such, reordering of the HF orbitals is often necessary. The boldfaced line illustrates the use of reordering directives to construct the desired active space, which, in this case, comprise of 5 occupied d-orbitals of $\mathrm{Mn}$ and 5 d-type virtual orbitals. The converged CASSCF wavefunction is then attached to end of this input file (omitted here due to length considerations). Note that some of the directives may have changed in the latest version of GAMESS, which is capable of performing parallel computations among a cluster of UNIX workstations.

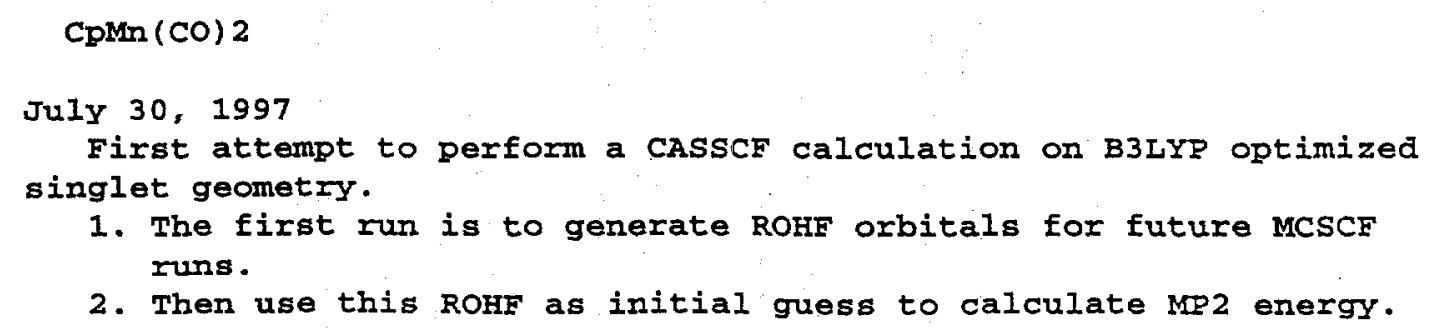




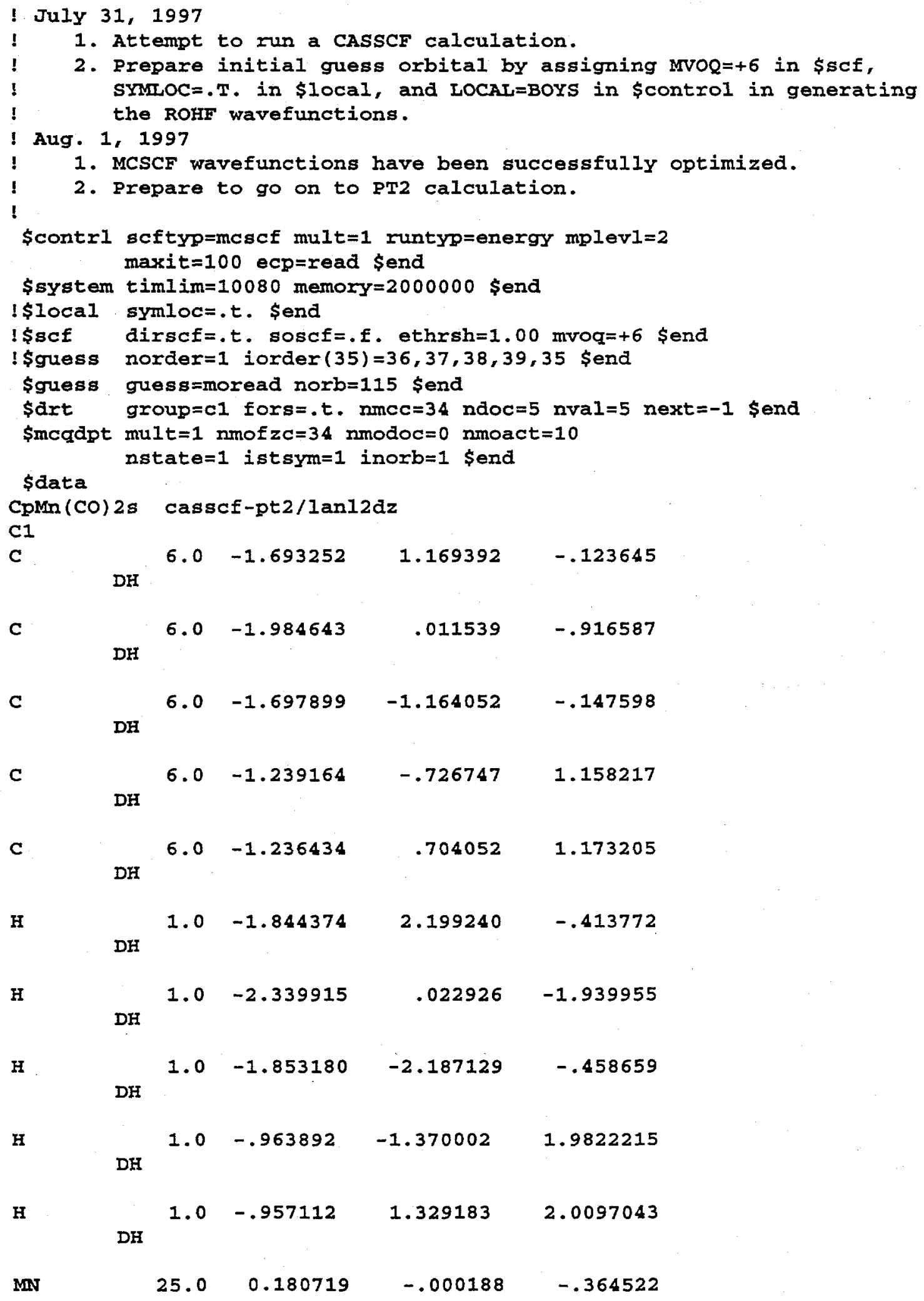




\begin{tabular}{|c|c|c|c|c|c|}
\hline $\mathbf{s}$ & 4 & & & & \\
\hline 1 & & 5.91 & 00000 & -0.37645080 & \\
\hline 2 & & 1.60 & 00000 & 0.77247890 & \\
\hline 3 & & 0.62 & 00000 & 0.47693460 & \\
\hline 4 & & 0.11 & 50000 & 0.00000000 & \\
\hline $\mathbf{s}$ & 4 & & & & \\
\hline 1 & & 5.91 & 00000 & 0.21199660 & \\
\hline 2 & & 1.60 & 00000 & -0.51994720 & \\
\hline 3 & & 0.62 & 00000 & -0.58576810 & \\
\hline 4 & & 0.11 & 50000 & 1.10039640 & \\
\hline $\mathbf{s}$ & 1 & & & & \\
\hline 1 & & 0.03 & 00000 & 1.00000000 & \\
\hline$P$ & 3 & & & & \\
\hline 1 & & 18.20 & 00000 & -0.04479010 & \\
\hline 2 & & 2.14 & 00000 & 0.62603110 & \\
\hline 3 & & 0.70 & 90000 & 0.46963290 & \\
\hline $\mathbf{P}$ & 1 & & & & \\
\hline 1 & & 0.06 & 00000 & 1.00000000 & \\
\hline $\mathbf{P}$ & 1 & & & & \\
\hline 1 & & 0.02 & 00000 & 1.00000000 & \\
\hline $\mathrm{D}$ & 4 & & & & \\
\hline 1 & & 32.27 & 00000 & 0.03415800 & \\
\hline 2 & & 8.87 & 00000 & 0.17611050 & \\
\hline 3 & & 2.89 & 00000 & 0.43942980 & \\
\hline 4 & & 0.87 & 10000 & 0.59432710 & \\
\hline$D$ & 1 & & & & \\
\hline 1 & & 0.21 & 00000 & 1.00000000 & \\
\hline C & & 6.0 & 1.358411 & 1.318936 & -.072387 \\
\hline & DH & & & & \\
\hline c & & 6.0 & 1.361026 & -1.316648 & -.072009 \\
\hline & DH & & & & \\
\hline 0 & & 8.0 & 2.137299 & 2.191364 & .120822 \\
\hline & DH & & & & \\
\hline 0 & & 8.0 & 2.141728 & -2.187407 & .121470 \\
\hline & $\mathrm{DH}$ & & & & \\
\hline send & & & & & \\
\hline secp & & & & & \\
\hline$C-E C P$ & NONE & & & & \\
\hline C-ECP & & & & & \\
\hline$C-\mathrm{ECP}$ & & & & & \\
\hline C-ECP & & & & & \\
\hline C-ECP & & & & & \\
\hline H-ECP & NONE & & & & \\
\hline H-ECP & & & & & \\
\hline $\mathrm{H}-\mathrm{ECP}$ & & & & & \\
\hline $\mathrm{H}-\mathrm{ECP}$ & & & & & \\
\hline H-ECP & & & & & \\
\hline $\mathrm{MN}-\mathrm{ECF}$ & P GEN & 10 & 2 & & \\
\hline
\end{tabular}




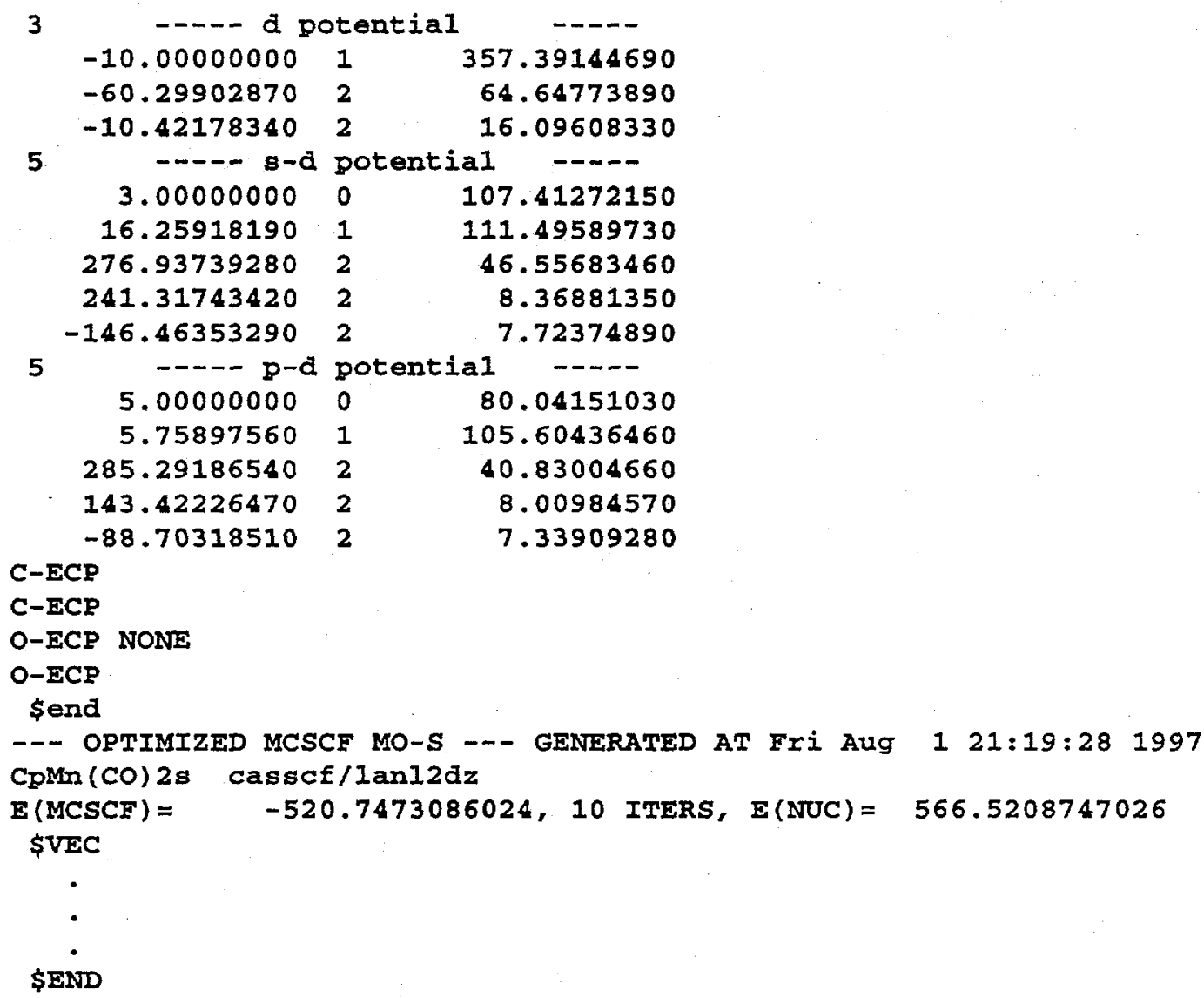

\subsubsection{The Input File for Calculating the CASSCF-PT2 Energy of the Triplet}

$$
\operatorname{CpMn}(\mathrm{CO})_{2} \text {. }
$$

The procedure is the same as described in the previous section, only that both the \$data and \$ecp segments are omitted.

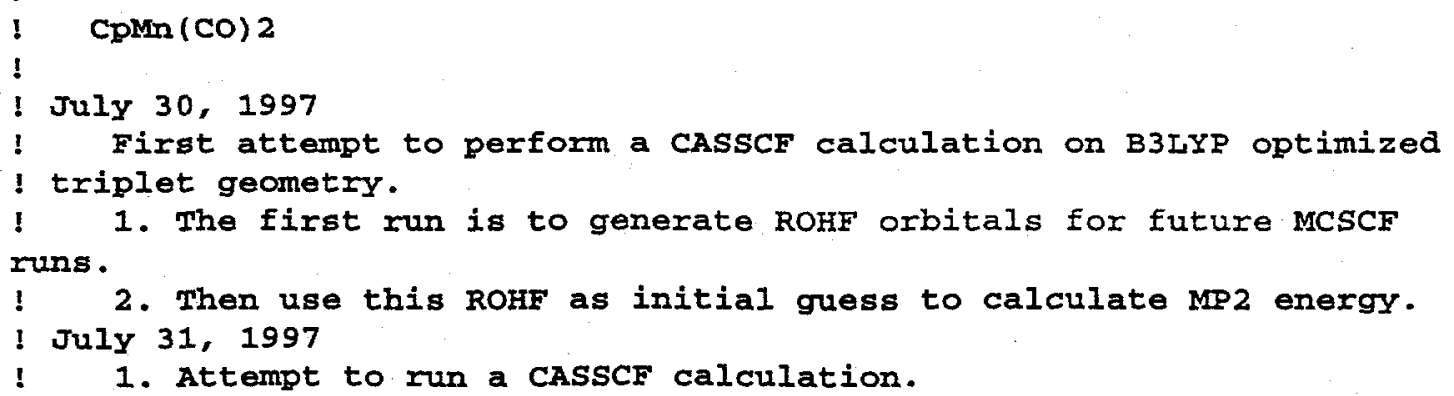




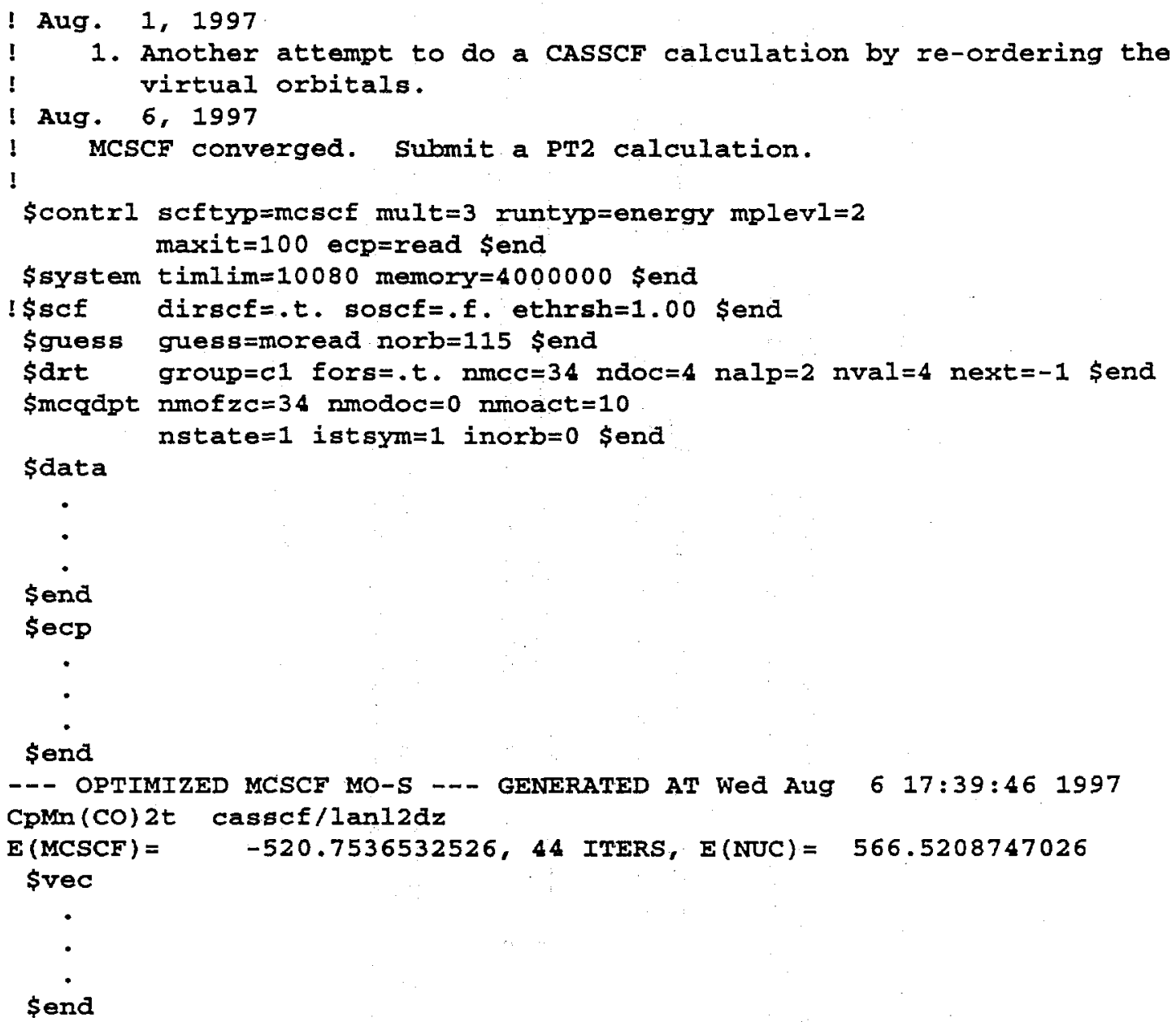

\subsection{Quantitative Considerations of the Triplet-Singlet Intersystem Crossing in}

Coordinatively Unsaturated Organometallic Compounds

In Chapter 5, a concerted spin cross over / solvation process (cf. Figure 7.1) has been proposed to explain the observed reaction dynamics of $\mathrm{Si}-\mathrm{H}$ bond activation by $\mathrm{CpMn}(\mathrm{CO})_{2}$. At the time of preparing that report, there was not enough data (only one data point, in fact) to formulate a quantitative model for such reactions. This section supplies quantitative and technical aspects in setting up such a model. 


\subsubsection{A Summary of Theoretical Models}

There have been several excellent reviews for the theory of intramolecular interconversion or electron transfer $[179,181-183]$. In summary, such a process can be considered from viewpoints of, for instance, a one-dimensional reaction coordinate: (i) a classical model; (ii) a semi-classical model (Landau-Zener coupling); and (iii) a quantum mechanical model. In (i), the rate can be expressed as

$$
\text { Equation 8.1 } \quad k_{c l}=v \exp \left(-\frac{\Delta E_{c l}}{R T}\right),
$$

where $v$ is the frequency of a pseudo-particle oscillating in the potential energy surface (PES) defined by the reaction coordinate (cf. the left-hand well of Figure 7.1), and $\Delta E_{c l}$ is the energy barrier defined by the crossing points of the two PESs. In (ii), the probability for $\gamma \rightarrow \beta$ transmission is

Equation 8.2

$$
P_{\gamma \rightarrow \beta}=1-\exp \left(-\frac{4 \pi^{2} H_{S T}^{2}}{h y\left|S_{T}-S_{S}\right|}\right),
$$

where $v$ is the velocity of the wave packet, $S_{T}$ and $S_{S}$ are the slopes of the triplet and singlet surfaces, respectively, and $H_{S T}$ is the coupling between the two states (cf. Figure 8.1). The rate for spin crossover is

$$
\text { Equation 8.3 } \quad k_{s c}=\kappa_{L z} k_{c l},
$$

where

Equation 8.4 $\quad \kappa_{L Z}=\frac{2 P_{\gamma \beta}}{1+P_{\gamma \beta}}$.

In (iii), the rate for intersystem crossing is,

$$
\text { Equation 8.5 } \quad k_{q m}=\frac{2 \pi}{\hbar} \frac{1}{\hbar \omega}\left|\left\langle\hat{H}_{s o}\right\rangle\right|^{2} G_{v i b} \text {, }
$$


where $\omega$ is the frequency of the triplet PES of Figure 7.1, $\hat{H}_{s o}$ is the spin-orbital operator, and $G_{v i b}$ is the vibrational overlap of the two states, $G_{v i b}=\left\langle\psi_{s} \mid \psi_{T}\right\rangle$.

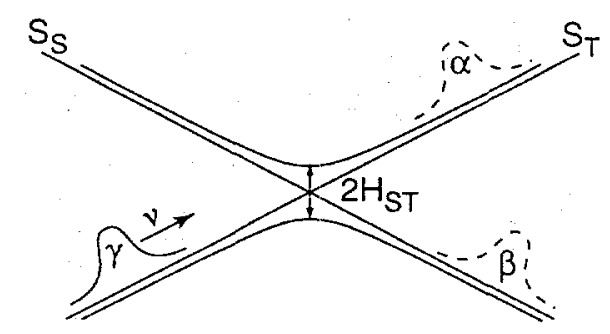

Figure 8.1 Illustraion of Landau-Zener spin crossover.

Following Figure 7.1, the intramolecular triplet-to-singlet spin crossover rate thus estimated can be viewed as the upper limit for that of solvation-assisted intersystem. Vibrational analyses of the singlet and triplet $\mathrm{CpMn}(\mathrm{CO})_{2}$ indicate that the most likely one-dimensional reaction coordinate is the bending motion of the carbonyls. The corresponding frequency and shift in normal coordinates are identified as $\sim 89 \mathrm{~cm}^{-1}$ and 3.93, respectively. With the singlet-triplet splitting energy calculated to be $\sim 10.9$ $\mathrm{kcal} / \mathrm{mol},{ }^{1}$ the overlapping factor $\mathrm{G}_{\mathrm{vib}}$ is found to be $\sim 6.7 \times 10^{-6}$ at room temperature. Finally, one calculates $\hat{H}_{s o}$ using ab initio methods and finds $\left|\hat{H}_{s o}\right| \sim 331 \mathrm{~cm}^{-1}$. Using these numbers, Equation 8.5 gives an upper limit of $100 \mathrm{ps}^{-1}$ for spin-crossover rate, consistent with experimental measurements of $70-90 \mathrm{ps}^{-1}$. While this estimate lends quantitative support for the measured dynamics, more data points are definitely needed in order to formulate a suitable model that includes the solvation process.

\subsubsection{The Input File for Calculating the Spin-Orbital Coupling Constant of $\mathrm{CpMn}(\mathrm{CO})_{2}$ Using MCSCF Wavefunctions}


For chemical species that exhibit strong spin-orbit coupling such as those being studied in this laboratory, single-determinant wavefunctions are no longer sufficient for an accurate description of the molecular properties. A multi-determinant wavefunction is necessary to calculate molecular properties that are quantitatively meaningful. The input file included in this section demonstrates the use of GAMESS to calculate the spin-orbit coupling constant between the singlet and triplet $\mathrm{CpMn}(\mathrm{CO})_{2}$ using the previously mentioned CASSCF wavefunctions. This is the best method available. In this input file, three configuration-interaction (CI) roots are computed and the spin-orbital calculation is carried out on the first one, the ground state. Experience shows that the number of roots one diagonalizes affects the relative energies between roots. In other words, one has to be very careful when computing excited-state properties. However, in this case, the spinorbital constants $\left|\hat{H}_{s o}\right|$ appear the same for diagonalizing one root $\left(331.23 \mathrm{~cm}^{-1}\right)$ or three roots $\left(331.22 \mathrm{~cm}^{-1}\right)$. Shown here is a calculation diagonlizing 3 roots, commanded by the "iroots $(1)=3,1$ " directive. Notice that the ordering of low-spin (\$cidrt1) and high-spin (\$cidrt2) wavefunctions must be followed.

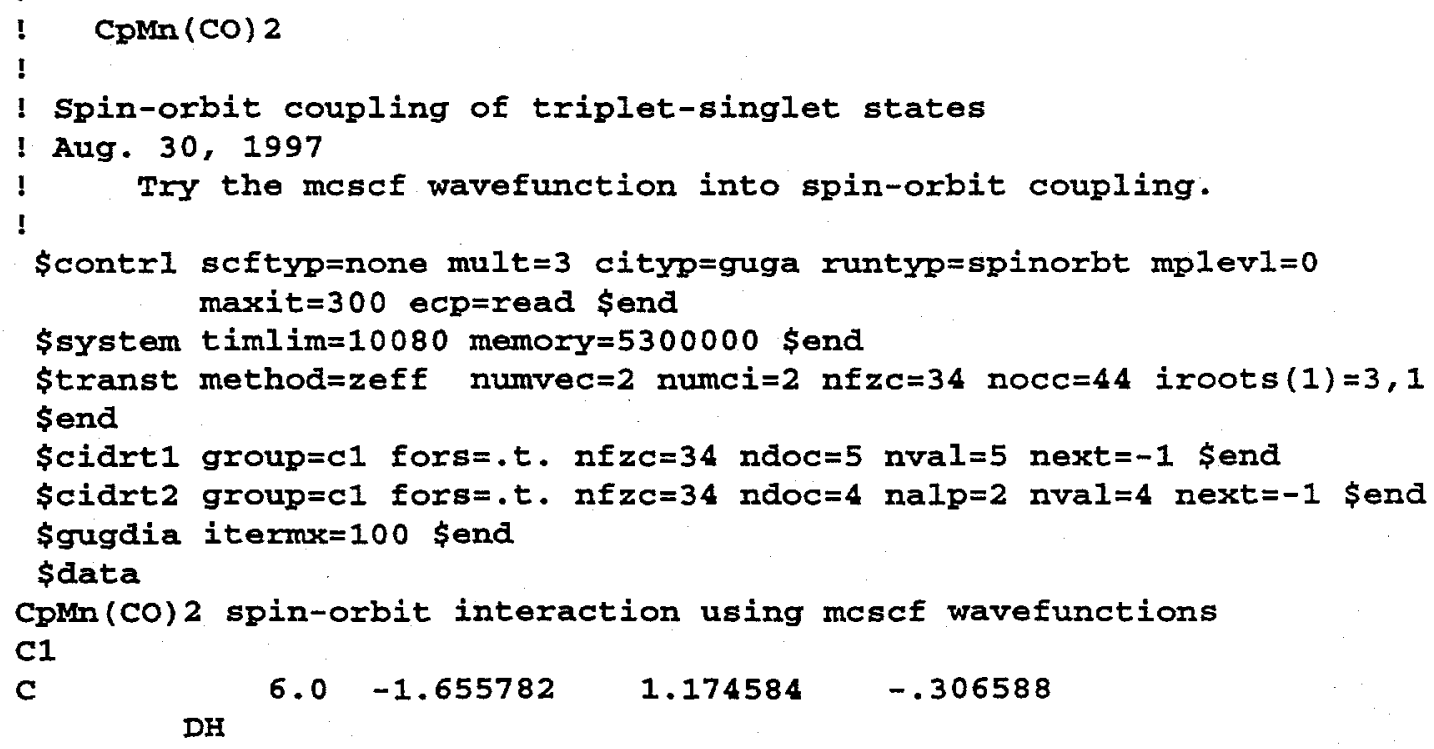

${ }^{1}$ Energy is calculated at the CASSCF-PT2 level of theory. 


\begin{tabular}{|c|c|c|c|c|c|c|}
\hline C & & $\mathrm{DH}$ & 6.0 & -1.706702 & .063358 & -1.211424 \\
\hline C & & $\mathrm{DH}$ & 6.0 & -1.637144 & -1.140211 & -.437748 \\
\hline C & & DEI & 6.0 & -1.600685 & -.773241 & .965821 \\
\hline C & & $\mathrm{DE}$ & 6.0 & -1.616435 & .655976 & 1.045136 \\
\hline $\mathbf{H}$ & & $\mathrm{DH}$ & 1.0 & -1.694780 & 2.219916 & -.581022 \\
\hline $\mathbf{H}$ & & $\mathrm{DH}$ & 1.0 & -1.749852 & .124541 & -2.290995 \\
\hline $\mathbf{H}$ & & DH & 1.0 & -1.665450 & -2.149299 & -.825587 \\
\hline H & & DH & 1.0 & -1.601322 & -1.4631 .85 & 1.798389 \\
\hline $\boldsymbol{H}$ & & DH & 1.0 & -1.622683 & 1.249882 & 1.948886 \\
\hline $\begin{array}{r}\mathbf{M N} \\
\mathbf{S}\end{array}$ & 4 & & 25.0 & .250854 & .001500 & -.001502 \\
\hline 1 & & & 5.91 & 400000 & -0.37645080 & \\
\hline 2 & & & 1.605 & 500000 & 0.77247890 & \\
\hline 3 & & & 0.62 & 600000 & 0.47693460 & \\
\hline 4 & & & 0.11 & 150000 & 0.00000000 & \\
\hline $\mathbf{S}$ & 4 & & & & & \\
\hline 1 & & & 5.91 & 400000 & 0.21199660 & \\
\hline 2 & & & 1.605 & 500000 & -0.51994720 & \\
\hline 3 & & & 0.626 & 600000 & -0.58576810 & \\
\hline 4 & & & 0.11 & 150000 & 1.10039640 & \\
\hline $\mathbf{S}$ & 1 & & & & & \\
\hline 1 & & & 0.03 & 800000 & 1.00000000 & \\
\hline $\mathbf{P}$ & 3 & & & & & \\
\hline 1 & & & 18.20 & 000000 & -0.04479010 & \\
\hline 2 & & & 2.14 & 100000 & 0.62603110 & \\
\hline 3 & & & 0.70 & 090000 & 0.46963290 & \\
\hline $\mathbf{P}$ & 1 & & & & & \\
\hline 1 & & & 0.06 & 900000 & 1.00000000 & \\
\hline $\begin{array}{l}P \\
1\end{array}$ & 1 & & 0.02 & 100000 & 1.00000000 & \\
\hline$D$ & 4 & & & & 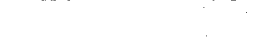 & \\
\hline 1 & & & 32.27 & 000000 & 0.03415800 & \\
\hline 2 & & & 8.87 & 500000 & $0.1761 \pm .050$ & \\
\hline 3 & & & 2.89 & 1000000 & 0.43942980 & \\
\hline 4 & & & 0.87 & 610000 & 0.59432710 & \\
\hline
\end{tabular}




\begin{tabular}{|c|c|c|c|c|c|}
\hline 1 & & 0.21 & 00000 & 1.00000000 & \\
\hline C & DH & 6.0 & 1.407347 & 1.423265 & -.009475 \\
\hline C & $\mathrm{DH}$ & 6.0 & 1.408235 & -1.416874 & -.009250 \\
\hline 0 & $\mathrm{DH}$ & 8.0 & 2.153162 & 2.340154 & -.014617 \\
\hline 0 & DH & 8.0 & 2.155553 & -2.332716 & -.014254 \\
\hline
\end{tabular}

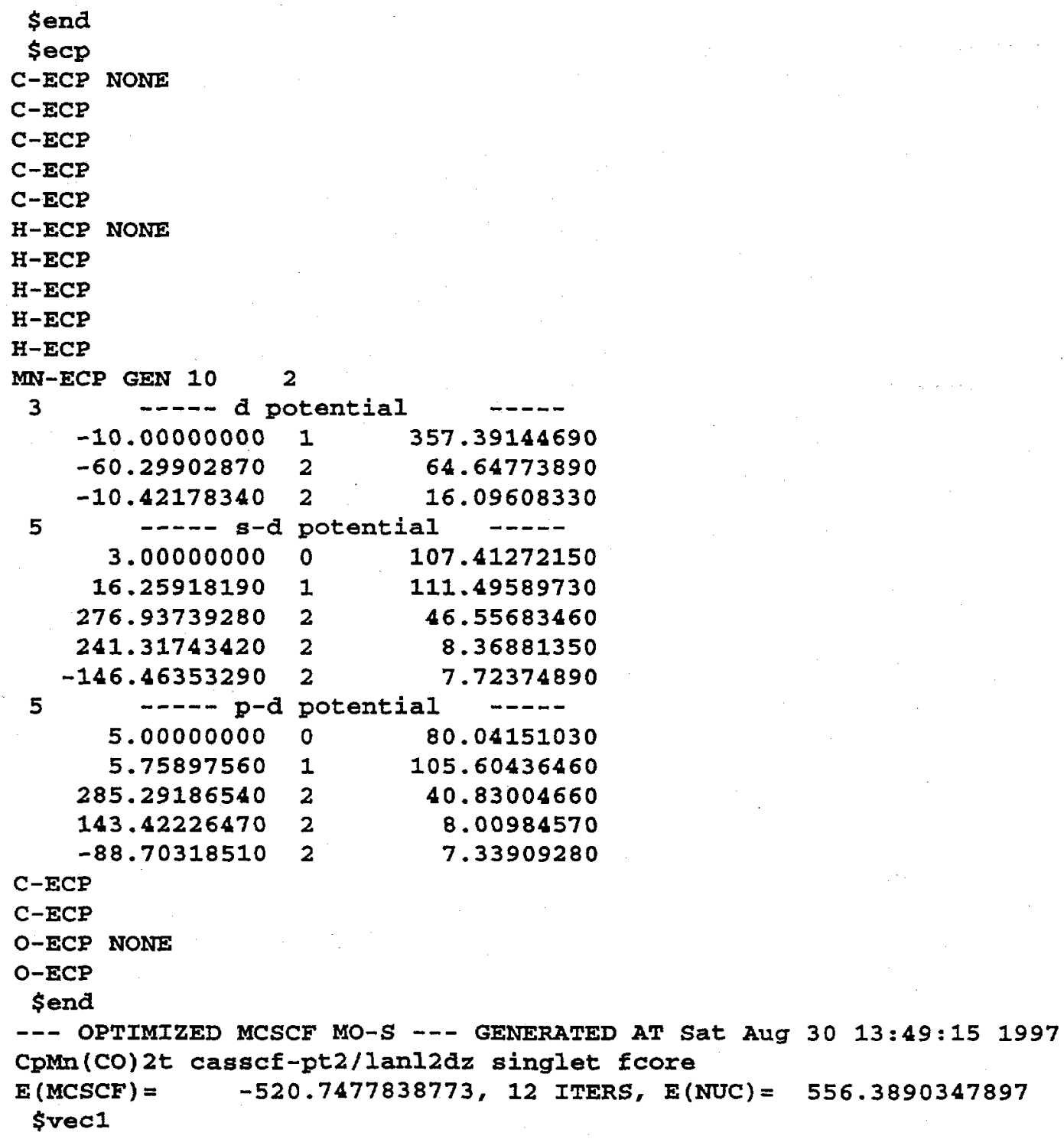




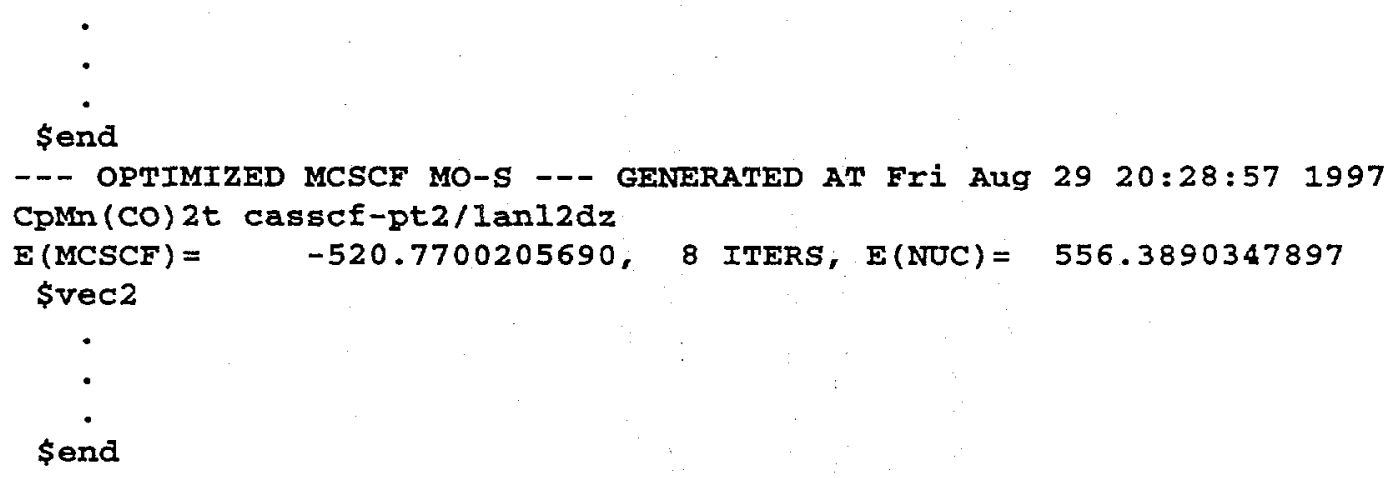

8.2.3 A MATLAB Script for Calculating the Overlap of Vibrational Wavefunctions between the Singlet and Triplet States

Figure 8.2 shows the vibrational quantum numbers that contribute to the rate constant calculated in Equation 8.5.

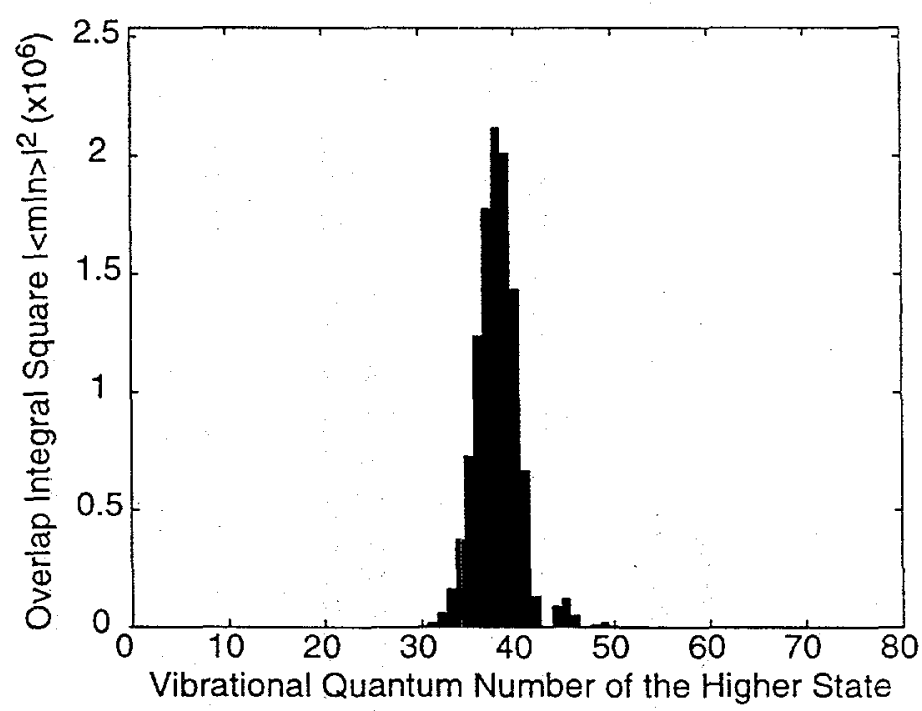

Figure 8.2 Graphical representation of the vibrational quantum numbers that contribute to the spin crossover rate.

$\%$

$\%$ File: fC.m

$\%$ Purpose: To calculate the overlap of two harmonic vibrational 


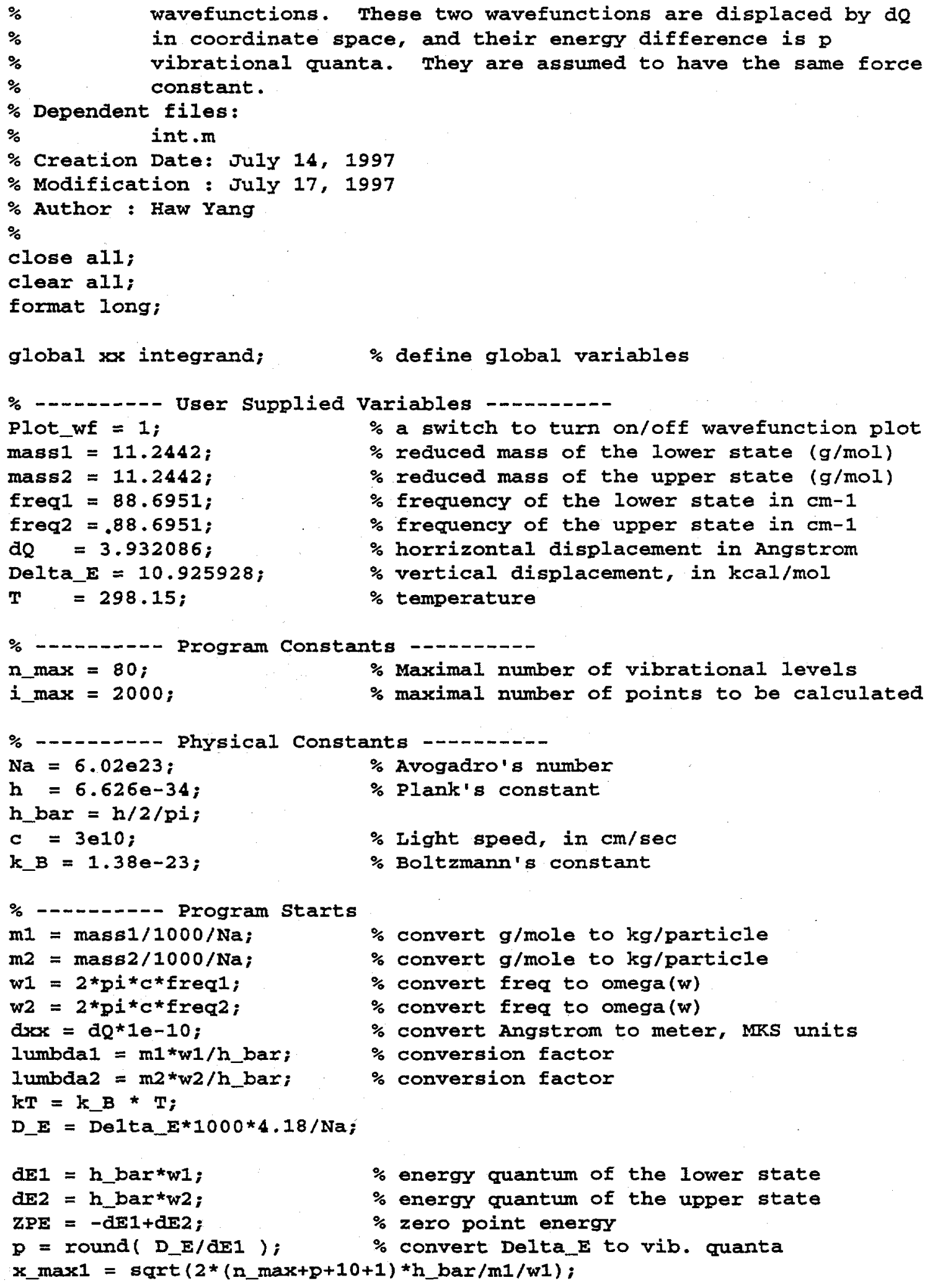


$\%$ classical turning point for lower

$\%$ calculated with 10 extra quanta

$x_{-} \max 2=\operatorname{sgrt}\left(2 *\left(n \_\max +10+1\right) * h \_b a r / m 2 / w 2\right) ;$

$\%$ classical turning point for upper

$x_{-} R=\max \left(x_{-} \max 1, x_{-} \max 2\right)+d x x_{i}$

$x_{-} L=-\max \left(x_{-} \max 1, x_{-} \max 2\right) ;$

$x \times x=x_{-} L:\left(x_{-} R-x_{-} L\right) /\left(i_{-} \max -1\right): x_{-} R ;$

$x 1$ = xx*sqrt(lumbda1); \%convert to normalized variable for Hermite

x2 = x*sqxt(1umbda2); \%convert to normalized variable for Hermite

$\mathrm{dx1}=\mathrm{dxx}{ }^{*}$ sqrt (lumbda1);

$\mathrm{dx2}=\mathrm{drox}$ sqrt (lumbda2);

$v 1=0.5 * m 1 * w 1 \wedge 2 * x \times \cdot \wedge 2 ; \%$ the potential energy function 1

$\mathrm{v} 2=0.5^{\star} \mathrm{m} 2 * \mathrm{w} 22^{\wedge} 2 *(x-x-d x x) . \wedge 2 ;$

$\%$ the potential energy function 2

$y 1=\exp (-0.5 * x 1 . \wedge 2) ; \quad \%$ the exponential function for $v i b$ wave 1

y2 $=\exp \left(-0.5^{\star}(x 2-d \times 2) . \wedge 2\right) ; \%$ the exponential function for vib wave 2

$A\left(i \_\max \right)=0 ; \quad \%$ reserve memory for the normalization constant

$H I$ (n_max+p,i_max) $=0 ; \%$ reserve memory for the Hermite polynomial 1

H2 (n_max $+p, i \_$max) $=0$; \% reserve memory for the Hermite polynomial 2

$\operatorname{FCs}\left(n_{-} \max \right)=0$;

Q $v i b=1$;

$\%$ reserve memory for the F-C factor

$\%$ vibrational partition function

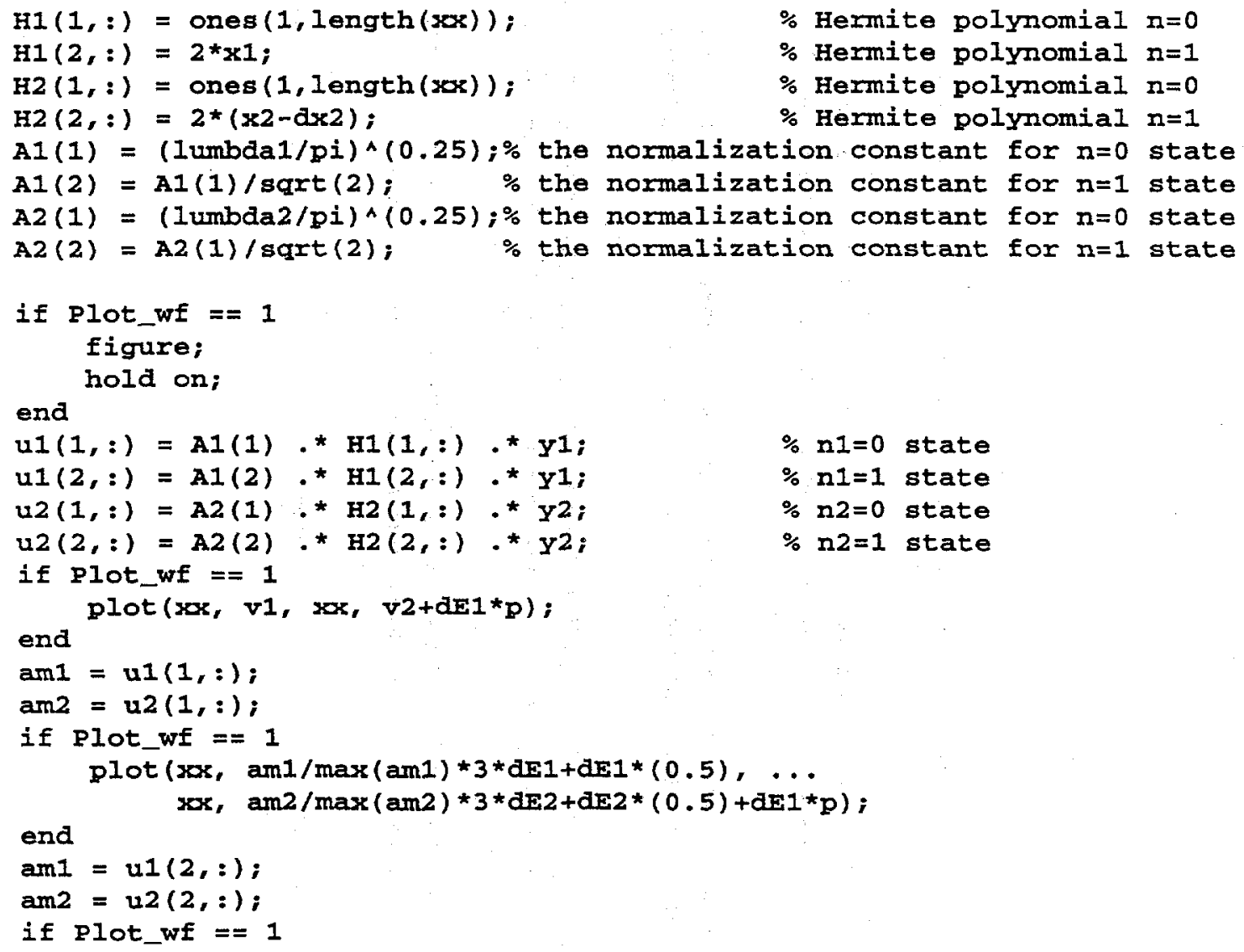

$\% \mathrm{nI}=0$ state

$\% \mathrm{nI}=1$ state

$\% \mathrm{n} 2=0$ state

$\% n 2=1$ state 


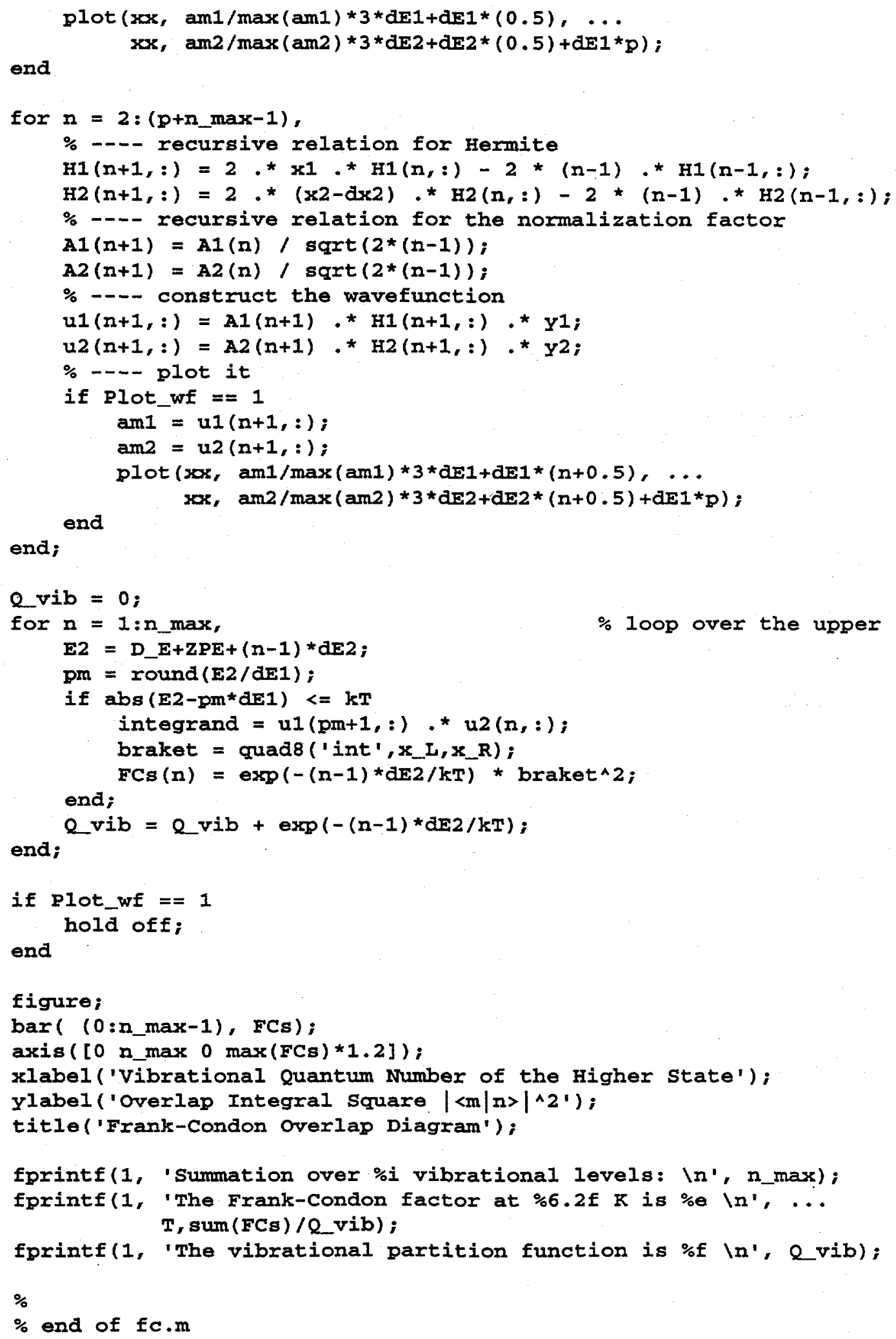


$\%$

\%ile: int.m

\% Rurpose: To supply a functional for integration of $\langle\mathrm{m} \mid \mathrm{n}\rangle$, where $|\mathrm{n}\rangle$ is a vibrational funciton.

$\%$ Dependent Files:

$\%$ fc.m

$\%$ Creation Date : July 14, 1997

\% Modification Date: July 14, 1997

$\%$ Author: Haw Yang

$\%$

function $z z z=\operatorname{int}(z)$

global $x \times$ integrand;

$z z z=$ interp1(xox, integrand, $z$ );

$\%$

$\%$ End of function int

$\%$ 


\section{BIBLIOGRAPHY}

[1] Collman JP, Hegedus LS, Norton JR, and Finke RG, "Principles and Applications of Organotransition Metal Chemistry." 1987.

[2] Masters C, "Homogeneous Transition-Metal Catalysis - A Gentle Art". London: Chapman and Hall, 1981.

[3] Lippard SJ and Berg JM, "Principles of Bioinorganic Chemistry". Mill Valley, California: University Science Books, 1994.

[4] Kubas GJ, Ryan RR, Swanson BI, Vergamini PJ, and Wasserman HJ, "Characterization of the First Examples of Isolable Molecular Hydrogen Complexes, $\mathrm{M}(\mathrm{CO})_{3}\left(\mathrm{PR}_{3}\right)_{2}\left(\mathrm{H}_{2}\right)(\mathrm{M}=\mathrm{Mo}, \mathrm{W} ; \mathrm{R}=\mathrm{Cy}, i$-Pr $)$. Evidence for a Side-on Bonded $\mathrm{H}_{2}$ Ligand." J. Am. Chem. Soc. 1984; 106: 451-452.

[5] Crabtree RH, "Dihydrogen Complexes: Some Structural and Chemical Studies." Acc. Chem. Res. 1990; 23(4): 95-101.

[6] Kubas GJ, "Molecular Hydrogen Complexes: Coordination of a $\sigma$ Bond to Transition Metals." Acc. Chem. Res. 1988; 21: 120-128.

[7] Hall C and Perutz RN, "Transition Metal Alkane Complexes." Chem. Rev. 1996; 96(8): 3125-3146.

[8] Musaev DG and Morokuma K, "Ab Initio Molecular Orbital Study of the Mechanism of $\mathrm{H}-\mathrm{H}, \mathrm{C}-\mathrm{H}, \mathrm{N}-\mathrm{H}, \mathrm{O}-\mathrm{H}$, and Si-H Bond Activation on Transient Cyclopentadienylcarbonylrhodium." J. Am. Chem. Soc. 1995; 117(2): 799-805.

[9] Stiegman AE and Tyler DR, "Reactivity of Seventeen- and Nineteen-Valence Electron Complexes in Organometallic Chemistry." Comments Inorg. Chem. 1986; $\underline{5}(5): 215-245$.

[10] Tyler DR, "19-Electron Organometallic Adducts." Acc. Chem. Res. 1991; 24: 325-331.

[11] Baird MC, "Seventeen-Electron Metal-Centered Radicals." Chem. Rev. 1988; 88: $1217-1227$. 
[12] Astruc D, "Nineteen-Electron Complexes and Their Role in Organometallic Mechanisms." Chem. Rev. 1988; $\underline{88(7): ~ 1189-1216 . ~}$

[13] Ohkubo K, Kanaeda H, and Tsuchihashi K, "An MO-theoretical Interpretation of the Reductive Cleavage of Organic Halides by Pentacyanocobaltate(II)." Bull. Chem. Soc. Jpn. 1973; 46 (10): 3095-3098.

[14] van Leeuwen PWNM, Morokuma K, and van Lenthe JH, "Theoretical Aspects of Homogeneous Catalysis". Vol. 18. Catalysis by Metal Complexes, ed. Ugo R and James BR. Boston: Kluwer Academic Publishers, 1995.

[15] Bromberg SE, Yang H, Asplund MC, Lian T, McNamara BK, Kotz KT, Yeston JS, Wilkens M, Frei H, Bergman RG, and Harris CB, "The Mechanism of a C-H Bond Activation Reaction in Room-Temperature Alkane Solution." Science 1997; 278: 260-263.

[16] Lian T, Bromberg SE, Yang H, Proulx G, Bergman RG, and Harris CB, "Femtosecond IR studies of Alkane C-H Bond Activation by Organometallic Compounds - Direct Observation of Reactive Intermediates in Room Temperature Solutions." J. Am. Chem. Soc. 1996; 118(15): 3769-3770.

[17] Yang H, Kotz KT, Asplund MC, and Harris CB, "Femtosecond Infrared Studies of Silane Silicon-Hydrogen Bond Activation." J. Am. Chem. Soc. 1997; 119(40): 9564-9565.

[18] Yang H, Asplund MC, Kotz KT, Wilkens MJ, Frei H, and Harris CB, "Reaction Mechanism of Silicon-Hydrogen Bond Activation Studied Using Femtosecond to Nanosecond IR Spectroscopy and Ab Initio Methods." J. Am. Chem. Soc. 1998; 120(39): 10154-10165.

[19] Yang H, Snee PT, Kotz KT, Payne CK, Frei H, and Harris CB, "Femtosecond Infrared Studies of a Prototypical One-Electron Oxidative-Addition Reaction: Chlorine Atom Abstraction by the $\operatorname{Re}(\mathrm{CO})_{5}$ Radical." J. Am. Chem. Soc. 1999; 121(39): 9227-9228. 
[20] Yang $\mathrm{H}$ and Harris $\mathrm{CB}$, "Probing Bond Activation Reactions with Femtosecond Infrared," in Book "Ultrafast Infrared and Raman Spectroscopy," Fayer MD, Editor. 2000, Marcel Dekker: New York.

[21] Yang H, Kotz KT, Asplund MC, Wilkens MJ, and Harris CB, "Ultrafast Infrared Studies of Bond Activation in Organometallic Complexes." Acc. Chem. Res. 1999; 32: 551-560.

[22] Bethune DS, "Dye cell design for high-power low-divergence excimer-pumped dye lasers." Appl. Opt. 1981; 20(11): 1897-1899.

[23] Wynne $\mathrm{K}$ and Hochstrasser RM, "The theory of ultrafast vibrational spectroscopy." Chem. Phys. 1995; 193: 211-236.

[24] Hamm P, "Coherent effects in femtosecond infrared spectroscopy." Chem. Phys. 1995; 200: 415-429.

[25] Kovalenko SA, Ernsting NP, and Ruthmann J, "Femtosecond Stokes shift in styryl dyes: Solvation or intramolecular relaxation?" J. Chem. Phys. 1997; 106(9): 3504-3511.

[26] Mukamel S, "Principles of nonlinear optical spectroscopy". New York: Oxford University Press, 1995.

[27] Akhremitchev B, Wang C, and Walker GC, "A femtosecond absorption spectrometer tunable from 50000 to $800 \mathrm{~cm}^{-1}$ : Nonlinear optics and pump/probe geometries." Rev. Sci. Instrum. 1996; 67(11): 3799-3805.

[28] Siegman AE, "Lasers". Mill Valley, California: University Science Books, 1986.

[29] Tokunaga E, Terasaki A, and Kobayashi T, "Femtosecond time-resolved dispersion relations studied with a frequency-domain interferometer." Phys. Rev. A 1993; 47(6): R4581-R4584.

[30] Ruhman S and Nelson KA, "Temperature-dependent molecular dynamics of liquid carbon disulphide: Polarization-selected impulsive stimulated lightscattering data and Kubo line shape analysis." J. Chem. Phys. 1991; 94(2): 859867. 
[31] Hattori $T$ and Kobayashi T, "Ultrafast optical Kerr dynamics studied with incoherent light." J. Chem. Phys. 1991; 94(5): 3332-3346.

[32] Ethepare J, Grillon G, Chambaret JP, Hamoniaux G, and Orszag A, "Polarization selectivity in time-resolved transient phase grating." Opt. Comm. 1987; 63(5): 329-334.

[33] Kalpouzos C, McMorrow D, Lotshaw WT, and Kenney-Wallace GA, "Femtosecond Laser-induced optical Kerr dynamics in $\mathrm{CS}_{2}$ /alkane binary solutions." Chem. Phys. 1988; $150(1,2): 138-146$.

[34] Shelton DP and Rice JE, "Measurements and calculations of the hyperpolarizabilities of atoms and small molecules in the gas phase." Chem. Rev. 1994; 94 : 3-29.

[35] Bishop DM, "Molecular vibrational and rotational motion in static and dynamic electric fields." Rev. Mod. Phys. 1990; $\underline{62(2): 343-374 . ~}$

[36] Föster $\mathrm{H}$ and Remmert $\mathrm{M}$, "Infrared studies on the interaction of $\mathrm{N}_{2} \mathrm{O}$ adsorbed in transition metal ion-exchange zeolite A." J. Mol. Struct. 1988; 174: 357-362.

[37] Villegas I and Weaver MJ, "Infrared spectroscopy of model electrochemical interfaces in ultrahigh vacuum: Roles of solvation in the vibrational stark effect." J. Phys. Chem. 1997; 101(30): 5842-5852.

[38] Lambert DK, "Vibrational Stark effect of $\mathrm{CO}$ on $\mathrm{Ni}(100)$, and $\mathrm{CO}$ in the aqueous double layer: Experiment, theory, and models." J. Chem. Phys. 1988; 89(6): 3847-3860.

[39] Kunimatsu K, “Adsorption of carbon monoxide on a smooth palladium electrode:

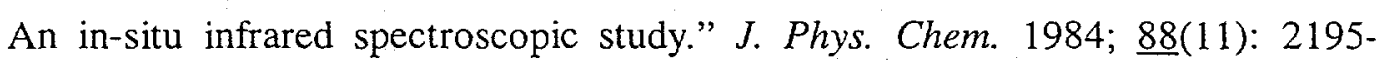
2200 .

[40] Lambert DK, "Observation of the first-order Stark effect of CO on Ni(110)." Phys. Rev. Lett. 1983; 50(26): 2106-2109.

[41] Lambert DK, "Observation of the first-order Stark effect of CO on Ni(110)." Phys. Rev. Lett. 1983; 51 (24): 2233. 
[42] Volk M, Kholodenko Y, Lu HSM, Gooding EA, DeGrado WF, and Hochstrasser RM, "Peptide conformational dynamics and vibrational Stark effect following photoinitiated disulfide cleavage." J. Phys. Chem. 1997; 101(42): 8607-8616.

[43] Lambert DK, "Stark effect of adsorbate vibrations." Solid State Comm. 1984; 51(5): 297-300.

[44] Hermansson $\mathrm{K}$ and Tepper $\mathrm{H}$, "Electric-field effects on vibrating polar molecules:

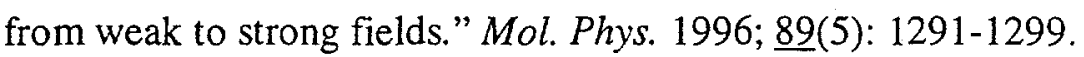

[45] Andrés JL, Martí J, Duran M, Lledós A, and Bertrán J, "Theoretical study of infrared spectra perturbed by uniform electric fields: $A b$ initio calculations on $\mathrm{H}_{2} \mathrm{O}, \mathrm{NH}_{3}, \mathrm{H}_{2} \mathrm{CO}$, and $\mathrm{C}_{2} \mathrm{H}_{4}$." J. Chem. Phys. 1991; 95(5): 3521-3527.

[46] Luis JM, Martí J, Duran M, and André JL, "Nuclear relaxation and vibrational contributions to the static electrical properties of polyatomic molecules: beyond the Hartree-Fock approximation." Chem. Phys. 1997; 217: 29-42.

[47] Andrés JL, Bertrán J, Duran M, and Martí J, "Perturbed infrared spectrum and vibrational contribution to electric properties of $\mathrm{CO}_{2}$ : An ab intio SCF study." J.

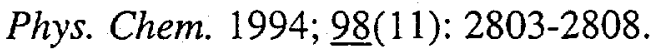

[48] Andrés JL, Duran M, Lledós A, and Bertrán J, "Calculation of the vibrational frequency and line strength versus applied field of carbon monoxide." Chem. Phys. 1991; 151: 37-43.

[49] Hermansson $\mathrm{K}$, "Electric-field effects on the $\mathrm{OH}$ vibrational frequency and infrared absorption intensity for water." J. Chem. Phys. 1993; 99(2): 861-868.

[50] Duran M, Andrés JL, Lledós A, and Bertrán J, "Influence of an external uniform electric field on harmonic vibrational frequencies. Analytic energy second derivatives for closed-shell restricted Hartree-Fock wave functions with an applied uniform electric field." J. Chem. Phys. 1989; 90(1): 328-333.

[51] Andrés JL, Bertrán J, Duran M, and Marti J, "Vibrational Stark Effect and Vibrational Static Electric Properties of $\mathrm{N}_{2}$ O." Int. J. Quant. Chem. 1994; 52: 9 15. 
[52] Bagus PS, Nelin CJ, Müller W, Philpott MR, and Seki H, "Field-induced vibrational frequency shifts of $\mathrm{CO}$ and $\mathrm{CN}$ chemisorbed on $\mathrm{Cu}(100)$." Phys. Rev.

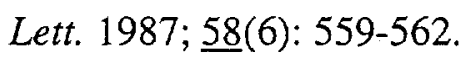

[53] Schmidt MW, Baldridge KK, Boatz JA, Elbert ST, Gordon MS, Jensen JH, Koseki S, Matsunaga N, Nguyen KA, Su SJ, Windus TL, Dupuis M, and Montgomery JA, "General Atomic and Molecular Electronic Structure System" J. Comput. Chem. 1993; 14: 1347-1363.

[54] Scott AP and Radom L, "Harmonic vibrational frequencies: An evaluation of Hartree-Fock, Møller-Plesset, quadratic configuration interaction, density functional theory, and semiempirical scale factors." J. Chem. Phys. 1996; 100(41): 16502-16513.

[55] Edwards DMF, Madden PA, and McDonald IR, "A computer simulation study of the dielectric properties of a model of methyl cyanide. I. The rigid dipole case." Mol. Phys. 1984; 51(5): 1141-1161.

[56] Nakagawa D and Shimanouchi T, Spect. Acta 1962; 18: 513.

[57] Steinfeld DI, "Molecules and Radiation". 2 ed, 1985.

[58] Weast RC, Lide DRL, Astle MJ, and Beyer WH, "CRC Handbook of Chemistry and Physics". 70 ed. Boca Raton: CRC Press, 1989-1990.

[59] Allen MP and Tildesley DJ, "Computer Simulation of Liquids". New York: Oxford Science Publications, 1986.

[60] Berendsen HJC, Postma JPM, van Gunsteren WF, DiNola A, and Haak JR, "Molecular Dynamics with Coupling to an External Bath." J. Chem. Phys. 1984; 81(8): 3684-3690.

[61] Wallqvist A and Teleman O, "Properties of Flexible Water Modelws." Mol. Phys. 1991; 74(3): 515-533.

[62] Tuckerman T, Berne BJ, and Martyna GJ, "Reversible Multiple Time Scale Molecular Dynamics." J. Chem. Phys. 1992; 97(3): 19902001. 
[63] Singer K, Taylor A, and Singer JVL, "Thermodynamic and Structural Properties of Liquids Modelled by '2-Lennard-Jones' Pair Potentials." Mol. Phys. 1977; 33(6): 1757-1795.

[64] Tran V and Schwartz BJ, "Role of Nonpolar Forces in Aqueous Solvation: Computer Simulation Study of Solvation Dynamics in Water Following Changes in Solute Size, Shape, and Charge." J. Phys. Chem. B 1999; 103(26): 5570-5580.

[65] Arndtsen BA and Bergman RG, "Selective Intermolecular Carbon-Hydrogen Bond Activation by Synthetic Metal Complexes in Homogeneous Solution." Acc. Chem. Res. 1995; 28: 154-162.

[66] Bengali AA, Arndtsen BA, Burger PM, Schultz RH, Weiller BH, Kyle KR, Moore CB, and Bergman RG, "Activation of Carbon-Hydrogen Bonds in Alkanes and Other Organic Molecules by $\operatorname{Ir}(\mathrm{I}), \mathrm{Rh}(\mathrm{I})$, and $\operatorname{Ir}(\mathrm{II})$ Complexes." Pure Appl. Chem. 1995; 67: 281-288.

[67] Bergman RG, "Activation of Alkanes with Organotransition Metal Complexes." Science 1984; 223: 902-908.

[68] Bergman RG, "A Physical Organic Road to Organometallic C-H Bond Oxidative Addition Reactions." J. Organomet. Chem. 1990; 400: 273-282.

[69] Crabtree RH, "The Organometallic Chemistry of Alkanes." Chem. Rev. 1985; 85: 245-269.

[70] Janowicz $\mathrm{AH}$ and Bergman $\mathrm{RG}$, "C-H Activation in Completely Saturated Hydrocarbons: Direct Observation of $\mathrm{M}+\mathrm{R}-\mathrm{H} \rightarrow \mathrm{M}(\mathrm{R})(\mathrm{H})$." J. Am. Chem. Soc. $1982 ; \underline{104}(1): 352-354$.

[71] Hoyano JK and Graham WAG, "Oxidative Addition of the Carbon-Hydrogen Bonds of Neopentane and Cyclohexane to a Photochemically Generated Iridium(I) Complex." J. Am. Chem. Soc. 1982; 104(13): 3723-3725.

[72] Bromberg SE, Lian TQ, Bergman RG, and Harris CB, "Ultrafast dynamics of $\mathrm{Cp} * \mathrm{M}(\mathrm{CO})_{2}(\mathrm{M}=\mathrm{Ir}, \mathrm{Rh})$ in solution: The origin of the low quantum yields for $\mathrm{C}$ H bond activation." J. Am. Chem. Soc. 1996; $118(8)$ : 2069-2072. And references therein. 
[73] Ghosh CK and Graham AG, "Efficient and Selective Carbon-Hydrogen Activation by a tris(Pyrazolyl)borate Rhodium Complex." J. Am. Chem. Soc. 1987; 109: 4726-4727.

[74] Ghosh CK and Graham AG, "A Rhodium Complex that Combines Benzene Activation with Ethylene Insertion - Subsequent Carbonylation and Ketone Formation." J. Am. Chem. Soc. 1989; 111: 375-376.

[75] Lees AJ and Purwoko AA, "Photochemical Mechanisms in Intermolecular C-H Bond Activation Reactions of Organometallic Complexes." Coord. Chem. Rev. 1994; 132: 155-160.

[76] Purwoko AA and Lees AJ, "Photochemistry and C-H Bond Activation Reactivity of $\left(\mathrm{HBPz}_{3}{ }_{3}\right) \mathrm{Rh}(\mathrm{CO})_{2}\left(\mathrm{Pz}^{*}=3,5\right.$-Dimethylpyrazolyl) in Hydrocarbon Solution." Inorg. Chem. 1995; 34: 424-425.

[77] Lee $M$ and Harris CB, "Ultrafast Studies of Transition-Metal Carbonyl Reactions in the Condensed Phase: Solvation of Coordinatively Unsaturated Pentacarbonyls." J. Am. Chem. Soc. 1989; 111(24): 8963-8965.

[78] Zaric S and Hall MB, "Prediction of the reactive intermediates in alkane activation by tris(pyrazolyl borate)rhodium carbonyl." J. Phys. Chem. A 1998; 102(11): 1963-1964.

[79] Schultz RH, Bengalli AA, Tauber MJ, Weiller BH, Wasserman EP, Kyle KR, Moore CB, and Bergman RG, "IR Flash Kinetic Spectroscopy of C-H Bond Activation of Cyclohexane- $\mathrm{D}_{0}$ and Cyclohexane- $\mathrm{D}_{12}$ by $\mathrm{Cp} * \mathrm{Rh}(\mathrm{CO})_{2}$ in Liquid Rare-Gases - Kinetics, Thermodynamics, and An Unusual Isotope Effect." J. Am. Chem. Soc. 1994; 116(16): 7369-7377.

[80] Asbury JB, Ghosh HN, Yeston JS, Bergman RG, and Lian TQ, "Sub-picosecond IR study of the reactive intermediate in an alkane $\mathrm{C}-\mathrm{H}$ bond activation reaction by CpRh(CO) 2." Organometallics 1998; 17(16): 3417-3419.

[81] Wick DD and Goldberg KI, "C-H Activation at Pt(II) to Form Stable Pt(IV) Alkyl Hydrides." J. Am. Chem. Soc. 1997; 119(42): 10235-10236. 
[82] Gutiérrez-Puebla E, Monge Á, Nicasio MC, Pérez PJ, Poveda ML, Rey L, Ruíz C, and Carmona E, "Vinylic $\mathrm{C}-\mathrm{H}$ bond Activation and Hydrogenation Reactions of $\operatorname{Tp} \operatorname{Tr}\left(\mathrm{C}_{2} \mathrm{H}_{4}\right)(\mathrm{L})$ Complexes." Inorg. Chem. 1998; 37(18): 4538-4546.

[83] Jiménez-Cataño R, Niu S, and Hall MB, "Theoretical Studies of Inorganic and Organometallic Reaction Mechanisms. 10. Reversal in Stability of Rhodium and Iridium $\eta^{2}$-Ethene and Hydridovinyl Complexes." Organometallics 1997; 16(9): 1962-1968.

[84] Moser WR and Slocum DW, "Homogeneous Transition Metal Catalyzed Reactions". Washington D.C.: American Chemical Society, 1992.

[85] Schubert U, " $\eta^{2}$ Coordination of Si-H $\sigma$ Bonds to Transition Metals." Adv. Organomet. Chem. 1990; 30: 151-187.

[86] Crabtree RH, "Aspects of Methane Chemistry." Chem. Rev. 1995; 95(4): 987 1007.

[87] Koga $\mathrm{N}$ and Morokuma $\mathrm{K}$, " $\mathrm{SiH}, \mathrm{SiSi}$, and $\mathrm{CH}$ Bond Activation by Coordinatively Unsaturated $\mathrm{RhCl}\left(\mathrm{PH}_{3}\right)_{2}$. Ab Initio Molecular Orbital Study." $J$. Am. Chem. Soc. $1993 ; \underline{115}(15): 6883-6892$.

[88] Young KM and Wrighton MS, "Temperature Dependence of the Oxidative Addition of Triethylsilane to Photochemically Generated $\left(\eta^{5}-\mathrm{C}_{5} \mathrm{Cl}_{5}\right) \mathrm{Mn}(\mathrm{CO})_{2}$." Organometallics 1989; $\underline{8}(4)$ : 1063-1066.

[89] Palmer BJ and Hill RH, "The Energetics of the Oxidative Addition of Trisubstituted Silanes to Photochemically Generated $\left(\eta^{5}-\mathrm{C}_{5} \mathrm{R}_{5}\right) \mathrm{Mn}(\mathrm{CO})_{2}$." Can. J. Chem. 1996; 74: 1959-1967.

[90] Hart-Davis AJ and Graham WAG, "Silicon-Transition Metal Chemistry. VI. Kinetics and Mechanism of the Replacement of Triphenylsilane by Triphenylphosphine in Hydridotriphenylsilyl $(\pi-$ cyclopentadienyl)dicarbonylmanganese." J. Am. Chem. Soc. 1971; 94(18): 43884393. 
[91] Hu S, Farrell GJ, Cook C, Johnston R, and Butkey TJ, "Rearrangement of $\eta^{5}$ $\mathrm{CpMn}(\mathrm{CO})_{2}\left(\mathrm{HSiEt}_{3}\right)$ : A Missing Step in the Energy Surface for the Oxidative Addition of Silane to $\mathrm{CpMn}(\mathrm{CO})_{2}$ (heptane)." Organometallics $1994 ; \underline{13}(11)$ : 4127-4128.

[92] Burkey TJ, "Thermochemistry of Silane Substitution of CO on Metal Carbonyl Complexes. Interaction of Silanes with Metal Centers." J. Am. Chem. Soc. 1990; 112(23): 8329-8333.

[93] Hester DM, Sun J, Harper AW, and Yang GK, "Characterization of the Energy Surface for the Oxidative Addition of Silanes to $\mathrm{CpMn}(\mathrm{CO})_{2}$ (heptane)." J. Am. Chem. Soc. $1992 ; 114(13): 5234-5240$.

[94] Breckenridge WH, "Activation of $\mathrm{H}-\mathrm{H}, \mathrm{Si}-\mathrm{H}$, and $\mathrm{C}-\mathrm{H}$ bonds by nsnp excited states of metal atoms." J. Phys. Chem. 1996; 100(36): 14840-14855.

[95] Dougherty TP, Grubbs WT, and Heilweil EJ, "Photochemistry of $\mathrm{Rh}(\mathrm{CO})_{2}$ (acetylacetonate) and Related Metal Dicarbonyls Studied by Ultrafast Infrared Spectroscopy." J. Phys. Chem. 1994; 98(38): 9396-9399.

[96] Arrivo SM, Dougherty TP, Grubbs WT, and Heilweil EJ, "Ultrafast Infrared Spectroscopy of Vibrational CO-Stretch Up-Pumping and Relaxation Dynamics of W(CO)6." Chem. Phys. Lett. 1995; 235: 247-254.

[97] Giordano PJ and Wrighton MS, Inorg. Chem. 1977; 16: 160.

[98] King JC, Zhang JZ, Schwartz BJ, and Harris CB, "Vibrational relaxation of $\mathrm{M}(\mathrm{CO})_{6}(\mathrm{M}=\mathrm{Cr}, \mathrm{Mo}, \mathrm{W})$ : effect of metal mass on vibrational cooling dynamics and non-Boltzmann internal energy distributions." J. Chem. Phys. 1993; 99(10): 7595-7601.

[99] Butler IS and Fenster AE, J. Organometl Chem. 1973; 51: 307.

[100] Hill RH and Wrighton MS, "Oxidative Addition of Trisubstituted Silanes to Potochemically Generated Coordinatively Uncaturated Spcies $\left(\eta^{4}\right.$ $\left.\mathrm{C}_{4} \mathrm{H}_{4}\right) \mathrm{Fe}(\mathrm{CO})_{2}, \quad\left(\eta^{5}-\mathrm{C}_{5} \mathrm{H}_{5}\right) \mathrm{Mn}(\mathrm{CO})_{2}$, and $\left(\eta^{6}-\mathrm{C}_{6} \mathrm{H}_{6}\right) \mathrm{Cr}(\mathrm{CO})_{2}$ and Related Molecules." Organometallics 1987 ; $6(3)$ : 632-638. 
[101] Zheng Y, Wang W, Lin J, She Y, and Fu K-J, "Time-Resolved Infrared Studies of Gas-Phase Coordinatively Unsaturated Photofragments (eta-5- $\left.\mathrm{C}_{5} \mathrm{H}_{5}\right) \mathrm{Mn}(\mathrm{CO})_{\mathrm{X}}(\mathrm{x}$ $=2$ and 1)." J. Phys. Chem. 1992; $96(19):$ 7650-7656.

[102] Bloyce PE, Mascetti J, and Rest AJ, "Photochemistry of tris(Dimethylpyrazolyl)Boratorhodium Dicarbonyl and bis(Dimethylpyrazolyl)-Boratorhodium Dicarbonyl Complexes in Low-Temperature Media at 12-298 K - Some Insights into C-H Activation Processes." J. Organomet. Chem. 1993; 444(1-2): 223-233.

[103] Ryther RJ and Weitz EJ, "Reaction-Kinetics of Coordinatively Unsaturated Iron Carbonyls Formed on Gas-Phase Excimer Laser Photolysis of $\mathrm{Fe}(\mathrm{CO})_{5}$." J. Phys. Chem. 1991; 95(24): 9841-9852.

[104] Maciejewski A, Jawoska-Augustniak A, Szeluga Z, and Karolczak J, "Determination of Ferrocene Triplet Lifetime by Measuring $\mathrm{T}_{1} \rightarrow \mathrm{T}_{1}$ Energy Transfer to Phenylosazone-d-glocise." Chem. Phys. Lett. 1988; 153(2-3): 227232.

[105] Bengali AA, Bergman RG, and Moore CB, "Evidence for the Formation of Free 16-Electron Species Rather than Solvate Complexes in the Ultraviolet Irradiation of $\mathrm{CpCo}(\mathrm{CO})_{2}$ in Liquefied Noble Gas Solvents." J. Am. Chem. Soc. 1995; 117(13): 3879-3880.

[106] Siegbahn PEM, "Comparison of the C-H Activation of Methane by $\mathrm{M}\left(\mathrm{C}_{5} \mathrm{H}_{5}\right)(\mathrm{CO})$ for $\mathrm{M}=$ Cobalt, Rhodium, and Iridium." J. Am. Chem. Soc. 1996; 118(6): 1487-1496.

[107] Rawlins KA and Lees AJ, "Photophysical Properties of $\mathrm{M}(\mathrm{CO})_{4}(\alpha, \alpha$ '-diimine) (M = Mo,W) Coplexes." Inorg. Chem. 1989; 28(11): 2154-2160. and references therein.

[108] Xie X and Simon JD, "Picosecond Time-Resolved Absorption Studies of the Solvation of $\mathrm{Cr}(\mathrm{CO})_{5}$ in Alcohols - A Unimolecular Kinetic-Model for the Formation of $\mathrm{Cr}(\mathrm{CO})_{5}(\mathrm{OHR})$ from Photogenerated $\mathrm{Cr}(\mathrm{CO})_{5}(\mathrm{ROH})$." J. Am. Chem. Soc. 1990; 112(3): 1130-1136. 
[109] Ladogana S, Nayak SK, Smit JP, and Dobson GR, "Mechanism of Linkage Isomerization in (eta(1)-5-chloropentene)pentacarbonylchromium(0)." Inorg. Chem. 1997; 36(4): 650-655.

[110] Klassen JK, Selke M, Sorensen AA, and Yang GK, "Metal-Ligand Bond Dissociation Energies in $\mathrm{CpMn}(\mathrm{CO})_{2} \mathrm{~L}$ Complexes." J. Am. Chem. Soc. 1990; 112: $1267-1268$.

[111] Jetz W and Graham WAG, Inorg. Chem. 1971; 10: 4.

[112] Wrighton MS and Schroeder MA, J. Am. Chem. Soc. 1974; 96: 6235.

[113] Smith RA and Bennett MJ, Acta Crystallogr., Sect. B 1977; 33: 1113.

[114] Schubert U, Ackermann K, and Wörle B, J. Am. Chem. Soc. 1982; 104: 7378.

[115] Schubert U, Scholz G, Müller J, Ackermann K, Wörle B, and Stansfield RFD, J. Organomet. Chem. 1986; 306: 303.

[116] Creaven BS, Dixon AJ, Kelly JM, Long C, and Poliakoff M, "Structure and Reactivity of (eta-5- $\left.\mathrm{C}_{5} \mathrm{H}_{5}\right) \mathrm{Mn}(\mathrm{CO})_{2}$ in Room-Temperature Solution - Evidence for Formation of A Dinuclear Intermediate Detected by Flash-Photolysis and Time-Resolved Infrared Spectroscopy." Organometallics 1987; $\underline{6}$ (12): 2600-2605.

[117] Yuzawa T, Kato C, George MW, and Hamaguchi H-O, "Nanosecond TimeResolved Infrared-Spectroscopy with A Dispersive Scanning Spectrometer." Appl. Spectrosc. 1994; 48(6): 684-690.

[118] Haas H and Sheline RK, J. Chem. Phys. 1967; 47: 2996.

[119] Wilson Jr. EB, Decius JC, and Cross PC, "Molecular Vibrations". New York: Dover, 1980.

[120] Dougherty TP and Heilweil EJ, "Transient Infrared Spectroscopy of ( $\eta^{5}$ $\left.\mathrm{C}_{5} \mathrm{H}_{5}\right) \mathrm{Co}(\mathrm{CO})_{2}$ Photoproduct reactions in Hydrocarbon Solutions." J. Chem. Phys. 1994; 100 (5): 4006-4009.

[121] Bode BM and Gordon MS, "MacMolPlt: A Graphical User Interface for GAMESS." J. Mol. Graphics Mod. 1998; 16: 133-138. 
[122] Brown CE, Ishikawa Y, Hackett PA, and Rayner DM, "Interaction of Alkanes with Unsaturated Metal Centers. 2. Complexes of Alkanes and Fluoroalkanes with $\mathrm{W}(\mathrm{CO}) 5$ in the Gas-Phase." J. Am. Chem. Soc. 1990; $112(7): 2530-2536$.

[123] Ishikawa $\mathrm{Y}$, Brown CE, Hackett PA, and Rayner DM, "Interaction of Alkanes with Unsaturated Metal Centers - The Complex W(CO) ${ }_{5} \mathrm{C}_{2} \mathrm{H}_{6}$ in the Gas-Phase." Chem. Phys. Lett. 1988; 150(6): 506-510.

[124] Zaric S and Hall MB, "Ab Initio Calculations of the Geometry and Bonding Energies of Alkanes and Fluoroalkanes Complexes with Tungsten Pentacarbonyl." J. Phys. Chem. A 1997; 101: 4646-4652. and references therein.

[125] Sun X-Z, Grills DC, Nikiforov SM, Poliakoff M, and George MW, "Remarkable Stability of $\left(\mathrm{eta}(5)-\mathrm{C}_{5} \mathrm{H}_{5}\right) \mathrm{Re}(\mathrm{CO})_{2} \mathrm{~L}$ ( $\mathrm{L}=\mathrm{n}$-heptane, $\mathrm{Xe}$, and $\mathrm{Kr}$ ): A TimeResolved Infrared Spectroscopic Study of $\left(\operatorname{eta}(5)-\mathrm{C}_{5} \mathrm{H}_{5}\right) \mathrm{Re}(\mathrm{CO})_{3}$ in Conventional and Supercritical Fluid Solution." J. Am. Chem. Soc. 1997; 119(32): 7521-7525.

[126] O'Connor JM and Casey CP, "Ring-Slippage Chemistry of Transition-Metal Cyclopentadienyl and Indenyl Complexes." Chem. Rev. 1987; 87(2): 307-318.

[127] Basolo F, "Ring-Slippage Mechanisms and Kinetics of CO Substitution of MetalCarbonyls." New J. Chem. 1994; 18(1): 19-24.

[128] Bengali AA, Bergman RG, and Moore CB, "Evidence for the Formation of Free 16-Electron Species Rather than Solvate Complexes in the Ultraviolet Irradiation of $\mathrm{CpCo}(\mathrm{CO})_{2}$ in Liquefied Noble Gas Solvents." J. Am. Chem. Soc. 1995; 117(13): 3879-3880.

[129] Abugideiri F, Keogh DW, and Poli R, "Stable 16-Electron, Paramagnetic Cyclopentadienylmolybdenum(II) Complexes." J. Chem. Soc., Chem. Commun. 1994(20): 2317-2318.

[130] Detrich JL, Reinaud OM, Rheingold AL, and Theopold KH, "Can Spin State Change Slow Organometallic Reactions?" J. Am. Chem. Soc. 1995; 117(47): $11745-11748$. 
[131] Poliakoff M and Weitz E, "Shedding Light on Organometallic Reactions - The Characterization of $\mathrm{Fe}(\mathrm{CO})_{4}$, A Prototypical Reaction Intermediate." Acc. Chem. Res. 1987 ; $20(11): 408-414$.

[132] Servaas PC, van Dijk HK, Snoeck TL, Stuhkens DJ, and Oskam A, Inorg. Chem. $1985 ; \underline{24}: 4494$.

[133] Jonas V and Thiel W, "Theoretical Study of the Vibrational Spectra of the Transition-Metal Carbonyl Hydrides $\mathrm{HM}(\mathrm{CO})_{5}(\mathrm{M}=\mathrm{Mn}, \mathrm{Re}), \mathrm{H}_{2} \mathrm{M}(\mathrm{CO})_{4}(\mathrm{M}=$ $\mathrm{Fe}, \mathrm{Ru}, \mathrm{Os})$, and $\mathrm{HM}(\mathrm{CO})_{4}(\mathrm{M}=\mathrm{Co}, \mathrm{Rh}, \mathrm{Ir})$." J. Chem. Phys. 1996; 105(9): 3636-3648.

[134] Zaric S, Couty M, and Hall MB, "Ab Initio Calculations of the Geometry and Vibrational Frequencies of the Triplet State of Tungsten Pentacarbonyl Amine: A Model for the Unification of the Preresonance Raman and the Time-Resolved Infrared Experiments." J. Am. Chem. Soc. 1997; 119(12): 2885-2888.

[135] Blomberg MRA, Siegbahn PEM, and Svensson M, "Mechanisms for the Reactions between Methane and the Neutral Transition Metal Atoms from Yttrium to Palladium." J. Am. Chem. Soc. 1992; 114(15): 6095-6102.

[136] Siegbahn PEM, "Different Electronic Structure Requirements on Precursors and Transition States for the Oxidative Reaction with Methane." J. Am. Chem. Soc. 1994; 116(22): 10124-10128.

[137] Carroll JJ, Haug KL, Weisshaar JC, Blomberg MRA, Siegbahn PEM, and Svensson M, "Gas Phase Reactions of Second-Row Transition Metal Atoms with Small Hydrocarbons: Experiment and Theory." J. Phys. Chem. 1995; 99(38): 13955-13969.

[138] Wrighton MS and Ginley DS, "Photochemistry of Metal-Metal Bonded Complexes. II. The Photochemistry of Rhenium and Manganese Carbonyl Complexes Containing a Metal-Metal Bond." J. Am. Chem. Soc. 1975; 97(8): 2065-2072.

[139] Firth S, Klotzbuecher WE, Poliakoff M, and Turner JJ, "Generation of $\operatorname{Re}_{2}(\mathrm{CO})_{9}\left(\mathrm{~N}_{2}\right)$ from $\operatorname{Re}_{2}(\mathrm{CO})_{10}$ : Identification of Photochemical Intermediates 
by Matrix Isolation and Liquid-Nobel-Gas Techniques." Inorg. Chem. 1987; 26(20): 3370-3375.

[140] Firth S, Hodges PM, Poliakoff M, and Turner JJ, "Comparative Matrix Isolation and Time-Resolved Infrared Studies on the Photochemistry of $\mathrm{MnRe}(\mathrm{CO}) 10$ and $\operatorname{Re}_{2}(\mathrm{CO})_{10}$ : Evidence for CO-Bridged $\mathrm{MnRe}(\mathrm{CO})_{9}$." Inorg. Chem: 1986; $25(25)$ : 4608-4610.

[141] Kim SK, Pedersen S, and Zewail AH, "Femtochemistry of Organometallics: Dynamics of Metal-Metal and Metal-Ligand Bond Cleavage in $\mathrm{M}_{2}(\mathrm{CO})_{10}$." Chem. Phys. Lett. 1995; 233: 500-508.

[142] Naqvi KR, Mork KJ, and Waldenstrfm S, "Diffusion-Controlled Reaction Kinetics. Equivalence of the Particle Pair Approach of Noyes and the Concentration Gradient Approach of Collins and Kimball." J. Phys. Chem. 1980; 84(11): 1315-1319. And references therein.

[143] Bultmann T and Ernsting NP, "Competition between Geminate Recombination and Solvation of Polar Radicals following Ultrafast Photodissociation of Bis( $p$ aminophenyl) Disulfide." J. Phys. Chem. 1996; 100(50): 19417-19424.

[144] Scott TW and Liu SN, "Picosecond Geminate Recombination of Phenylthiyl Free-Radical Pairs." J. Phys. Chem. 1989; 93: 1393.

[145] Churchill MR, Amoh KN, and Wasserman HJ, "Redetermination of the Crystal Structure of Dimanganese Decacarbonyl and Determination of the Crystal Structure of Dirhenium Decacarbonyl. Revised Values for the Mn-Mn and Re-Re Bond Lengths in $\mathrm{Mn}_{2}(\mathrm{CO})_{10}$ and $\operatorname{Re}_{2}(\mathrm{CO})_{10}$." J. Inorg. Chem. 1981; 20(5): 1609-1611.

[146] Hammond GS, "A Correlation of Reaction Rates." J. Am. Chem. Soc. 1954; 77: 334-338.

[147] Herrick RS, Herrinton TR, Walker HW, and Brown TL, "Rates of Halogen Atom Transfer to Manganese Carbonyl Radicals." Organometallics 1985; 4(1): 42-45. 
[148] Lee K-W and Brown TL, "On the Nature of Halogen Atom Transfer Reactions of $\operatorname{Re}\left(\mathrm{CO}_{4}\right)$ L Radicals." J. Am. Chem. Soc. 1987; 109(11): 3269-3275.

[149] Lian T, Bromberg SE, Asplund MC, Yang H, and Harris CB, "Femtosecond Infrared Studies of the Dissociation and Dynamcis of Transition Metia Carbonyls in Solution." J. Phys. Chem. 1996; 100(29): 11994-12001.

[150] Sun H and Frei H, "Time-resolved step-scan Fourier transform infrared spectroscopy of triplet excited duroquinone in a zeolite." J. Phys. Chem. B 1997; 101(2): 205-209.

[151] Becke AD, "Density-Functional Thermochemistry. III. The Role of Exact Exchange." J. Chem. Phys. 1993; $\underline{98(7): ~ 5648-5652 . ~}$

[152] Lee C, Yang W, and Parr RG, "Development of the Colle-Salvetti CorrelationEnergy Formula into a Functional of the Electron-Density." Phys. Rev. 1988; B41(2): 785-789.

[153] Stephens PJ, Devlin FJ, Chabalowski CF, and Frisch MJ, "Ab Initio Calculation of Vibrational Absorption and Circular Dichroism Spectra Using Density Functional Force Fields." J. Phys. Chem. 1994; 98(10): 11623-11627.

[154] Ricca A and Jr. CWB, "A Comparison of Density Functional Theory with $A b$ Initio Approaches for Systems Involving First Transition Row Metals." Theor. Chim. Acta. 1995; 92: 123-131.

[155] Glukhovtsev MN, Bach RD, and Nagel CJ, "Performance of the B3LYP/ECP DFT Calculations of Iron-Containing Compounds." J. Phys. Chem. 1997; 101(3): 316-323.

[156] Francl MM, Petro WJ, Hehre WJ, Binkley JS, Gordon MS, DeFrees DJ, and Pople JA, "Self-Consistent Molecular Orbital Methods. XXIII. A PolarizationType Basis Set for Second-Row Elements." J. Chem. Phys. 1982; 77(7): 36543665 .

[157] Hehre WJ, Ditchfield R, and Pople JA, "Gaussian-Type Basis Set for Use in

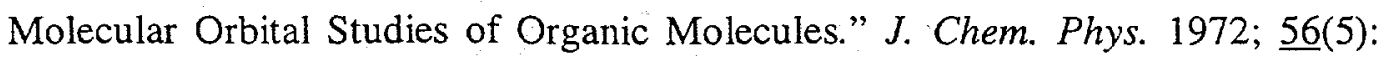
2257-2261. 
[158] Hay PJ and Wadt WR, "Ab initio Effective Core Potentials for Molecular Calculations. Potentials for $\mathrm{K}$ to $\mathrm{Au}$ including the outermost core orbitals." $J$. Chem. Phys. $1985 ; \underline{82}(1): 299-310$.

[159] Lendvay G and Mayer I, "Some Difficulties in Computing BSSE-Corrected Potential Surfaces of Chemical Reactions." Chem. Phys. Lett. 1998; 297: 365373. and references therein.

[160] "Jaguar 3.5". Portland, OR: Schroedinger, Inc., 1998.

[161] Hippler H, Otto B, Schroeder J, and Troe J, "Diffusion Controlled Atom Recombination and Photolytic Cage Effect of Halogen in Compressed Gases and Liquids." Ber. Bunsenges. Physik. Chem. 1985; 89: 240.

[162] Otto B, Schroeder J, and Troe J, "Photolytic Cage Effect and Atom Recombination of Iodine in Compressed Gases and Liquids: Experiments and Simple Models." J. Chem. Phys. 1984; $\underline{81}$ (1): 202-213.

[163] Schroeder J and Troe J, "Elementary Reactions in the Gas-Liquid Transition Range." Ann. Rev. Phys. Chem. 1989; 38: 163.

[164] Carslaw HS and Jaeger JC, "Conduction of Heat in Solids", 1959.

[165] Straub JE and Karplus M, "Molecular dynamics study of the photodissociation of carbon monoxide from myoglobin: ligand dynamics in the first 10 ps." Chem. Phys. $1991 ; 158(2): 221-248$.

[166] Sarakha $M$ and Ferraudi G, "Electron-Transfer Reactions of $\operatorname{Re}(\mathrm{CO})_{5}$ : AtomTransfer-Concerted Mechanism vs. Bimetallic Intermediate Formation." Inorg. Chem. 1996; $\underline{35}(2): 313-317$.

[167] Meckstroth WK, Reed DT, and Wojcicki A, "Further Pulse Radiolysis Studies of Decacarbonyldimanganese $(0)$ and Decacarbonyldirhenium $(0)$. Temperature and Solvent Effects." Inorg. Chim. Acta 1985; 105: 147-151.

[168] Tolman CA, "The 16 and 18 Electron Rule in Organometallic Chemistry and Homogeneous Catalysis." Chem. Soc. Rev. 1972; 1: 337-353. 
[169] Poli R, "Open-Shell Organometallics as a Bridge bewteen Werner-Type and LowValent Organometallic Complexes. The Effect of the Spin State on the Stability, Reactivity, and Structure." Chem. Rev. 1996; 96(6): 2135-2204.

[170] Shaik S, Filatov M, Schröder D, and Schwarz H, "Electronic Structure Makes a Difference: Cytochrome P-450 Mediated Hydroxylations of Hydrocarbons as a Two-State Reactivity Paradigm." Chem. Eur. J. 1998; 4(2): 193-199.

[171] Poli R and Smith KM, "Spin State and Ligand Dissociation in [ $\mathrm{CpCoL}_{2}$ ] Complexes ( $\left.\mathrm{L}=\mathrm{PH}_{3}, \mathrm{H}_{2} \mathrm{C}=\mathrm{CH}_{2}\right)$ : A Computational Study." Eur. J. Inorg. Chem. 1999: 877-880.

[172] Smith KM, Poli R, and Legzdins P, "A Computational Study of Two-State Conformational Change in 16-Electron $[\mathrm{CpW}(\mathrm{NO})(\mathrm{L})]$ Complexes $\left(\mathrm{L}=\mathrm{PH}_{3}, \mathrm{CO}\right.$, $\mathrm{CH}_{2}, \mathrm{HCCH}, \mathrm{H}_{2} \mathrm{CCH}_{2}$ )." Chem. Eur. J. 1999; $\underline{5}(5):$ 1598-1608.

[173] Keogh DW and Poli R, "Spin State Change in Organometallic Reactions. Experimental and MP2 Theoretical Studies of the Thermodynamics and Kinetics of the $\mathrm{CO}$ and $\mathrm{N}_{2}$ Addition to Spin Triplet $\mathrm{Cp} * \mathrm{MoCl}\left(\mathrm{PMe}_{3}\right)_{2}$." J. Am. Chem. Soc. $1997 ; 119(10): 2516-2523$.

[174] Poli R, "Molybdenum Open-Shell Organometallics. Spin State Changes and Pairing Energy Effects." Acc. Chem. Res. 1997; 30(12): 494-501.

[175] Lenges CP, White PS, and Brookhart M, "Mechanistic and Synthetic Studies of the Addition of Alkyl Aldehydes to Vinylsilanes Catalyzed by Co(I) Complexes." J. Am. Chem. Soc. 1998; 120 (28): 6965-6979.

[176] Lenges $\mathrm{CP}$ and Brookhart $\mathrm{M}$, "Co(I)-Catalyzed Inter- and Intramolecular Hydroacylation of Olefins with Aromatic Aldehydes." J. Am. Chem. Soc. 1997; 119(13): 3165-3166.

[177] Lenges CP, Brookhart M, and Grant BE, "H/D Exchange Reactions between $\mathrm{C}_{6} \mathrm{D}_{6}$ and $\mathrm{C}_{5} \mathrm{Me}_{5} \mathrm{Co}\left(\mathrm{CH}_{2}=\mathrm{CHR}\right)_{2}\left(\mathrm{R}=\mathrm{H}, \mathrm{SiMe}_{3}\right)$ : Evidence for Oxidative Addition of $\mathrm{C}_{\mathrm{sp} 2}-\mathrm{H}$ Bonds to the $\left[\mathrm{C}_{5} \mathrm{Me}_{5}(\mathrm{~L}) \mathrm{Co}\right]$ Moiety." J. Organomet. Chem. 1997; 528: 199-203. 
[178] Snee PT, Yang H, Kotz KT, Payne CK, and Harris CB, "Femtosecond Infrared Studies of the Mechanism of Silicon-Hydrogen Bond Activation by $\eta^{5}$ CpV(CO)4." J. Phys. Chem. 1999. Accepted for publication.

[179] Hauser A, "Intersystem Crossing in Iron(II) Coordination Compounds: A Model Process Bewteen Classical and Quantum Mechanical Behavior." Comments Inorg. Chem. 1995; 17(1): 17-40. And references therein.

[180] Kotz KT, Yang H, Snee PT, Payne CK, and Harris CB, "Femtosecond Infrared Studies of Ligand Rearrangement Reaction: Silyl Hydride Products from Group 6 Carbonyls." J. Organomet. Chem. 1999. Accepted for publication.

[181] Buhks E, Navon G, Bixon M, and Jortner J, "Spin conversion processes in solutions." J. Am. Chem. Soc. 1980; 102(9): 2918-2923.

[182] Lin SH, "Rate of Interconversion of Electronic and Vibrational Energy." J. Chem. Phys. 1966; 44(10): 3759-3767.

[183] Sutin N, "Theory of Electron Transfer Reactions: Insights and Hindsights," in Book "Prog. Inorg. Chem.," Lippard SJ, Editor. 1983, John Wiley \& Sons, Inc. p. 441-498. 